\title{
Interplays and feedback loops of oncogenic signaling pathways in B cell non-Hodgkin lymphoma
}

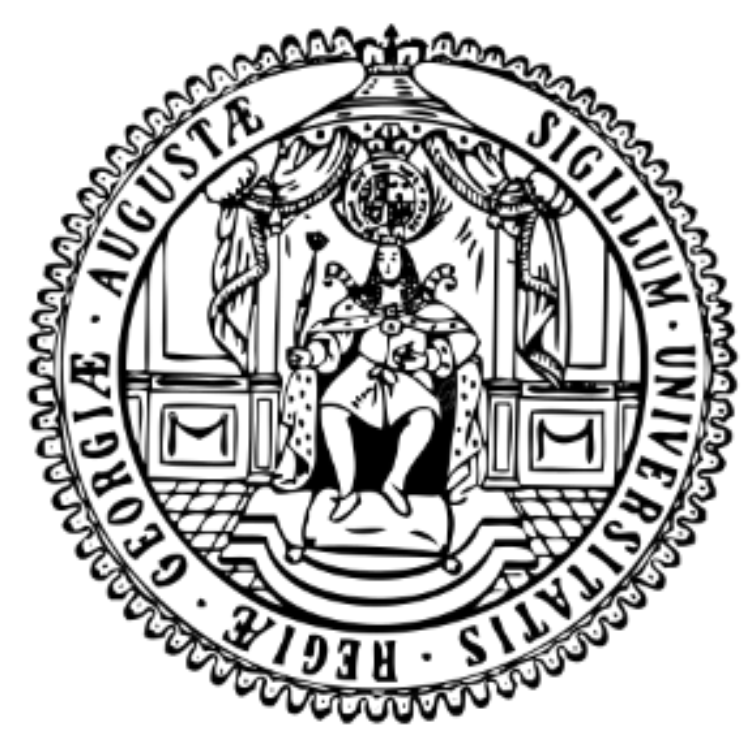

\section{Dissertation}

for the award of the degree

"Doctor rerum naturalium (Dr. rer. nat.)"

at the Georg-August-University Goettingen

within the doctoral degree program Molecular Medicine

of the Georg-August-University School of Science (GAUSS)

submitted by

\section{Isabel Rausch}

born in Bremen 



\section{Thesis Advisory Committee}

Prof. Dr. Dieter Kube

(Supervisor)

Prof. Dr. Heidi Hahn

Prof. Dr. mult. Thomas Meyer
Clinic of Hematology and medical Oncology

University Medical Centre Goettingen

Institute of Human Genetics

University Medical Centre Goettingen

Clinic of Psychosomatic Medicine and Psychotherapy

University Medical Centre Goettingen

\section{Members of the examination board}

Prof. Dr. Dieter Kube

(Referee)

Prof. Dr. Heidi Hahn

(Co-referee)

Prof. Dr. mult. Thomas Meyer

Prof. Dr. Ralf Dressel

Prof. Dr. Peter Burfeind

Prof. Dr. Jörg Großhans
Clinic of Hematology and medical Oncology

University Medical Centre Goettingen

Institute of Human Genetics

University Medical Centre Goettingen

Clinic of Psychosomatic Medicine and Psychotherapy

University Medical Centre Goettingen

Institute for Cellular and Molecular Immunology

University Medical Centre Goettingen

Institute of Human Genetics

University Medical Centre Goettingen

Institute for Developmental Biochemistry

University Medical Centre Goettingen

Date of the oral examination: 


\section{Affidavit}

By this, I declare that I independently authored the presented thesis:

"Interplays and feedback loops of oncogenic signaling pathways in B cell non-Hodgkin lymphoma"

and that I did not use other auxiliary means than indicated. Paragraphs that are taken from other publications, by wording or by sense, are marked in every case with a specification of the literary source.

Furthermore, I declare that I carried out the scientific experiments following the principles of Good Scientific Practice according to the valid "Richtlinien der Georg-August-Universität Göttingen zur Sicherung guter wissenschaftlicher Praxis".

Isabel Rausch

Goettingen, January 2019 


\section{List of Publications}

Articles in preparation:

Rausch I., Klinger B., Witzel F., Sieber A., Kutz H., Kieser A., Blüthgen N., Kube D. (2018): Quantitative modelling of $B$ cell signaling unveils global feedback loops and crosstalk from p38 MAPK to ERK signaling.

Rausch I., Feist M., von Bonin F., Kube D. (2018): NF-kB activation reduces migration capacity despite STAT3 activation in non-Hodgkin lymphoma.

Abstracts:

Rausch I., Hand E., Kutz H., Klinger B., Witzel F., Sieber A., Kieser A., Beissbarth T., Spang R., Blüthgen N., Kube D. (2017): Interaction of signalling pathways in Burkitt lymphoma. Poster presentation at the EKFS Cancer Symposium, Goettingen, Germany, 15-17 Nov 2017.

Rausch I., Klinger B., Witzel F., Sieber A., Kutz H., Kieser A., Blüthgen N., Kube D. (2018): Quantitative modelling of $B$ cell signalling unveils global feedback loops and crosstalk from p38 MAPK to ERK signalling. Poster presentation at the STS $-22^{\text {nd }}$ Joint Meeting "Signal Transduction - Receptors, Mediators and Genes", Weimar, Germany, 05-07 Nov 2018. 


\section{Table of Contents}

Acknowledgment . 1

Abstract II

List of Tables III

List of Figures IV

Abbreviations V

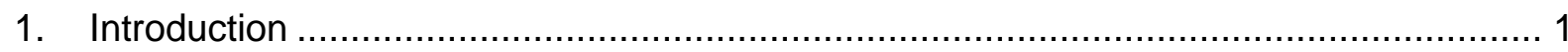

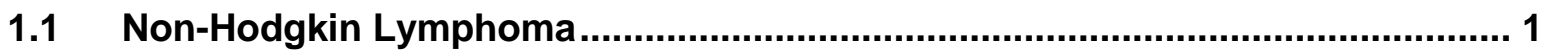

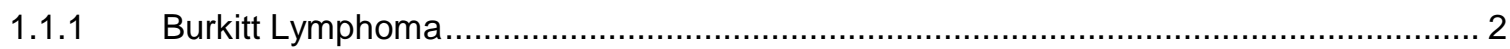

1.1.2 Diffuse Large B cell Lymphoma .......................................................................... 3

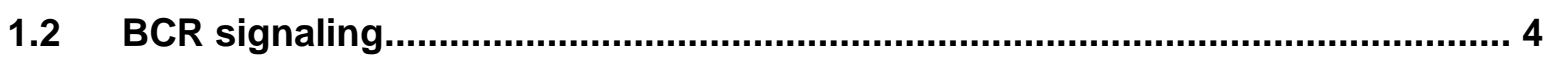

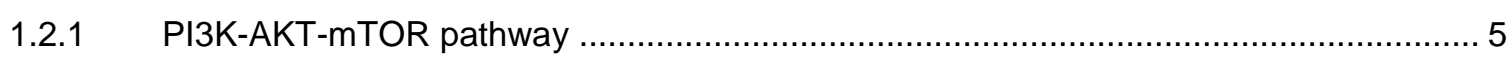

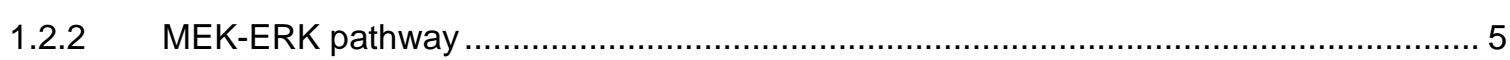

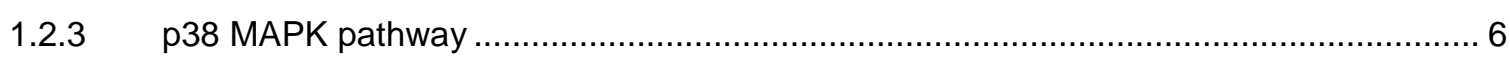

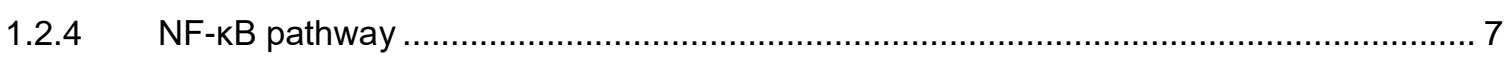

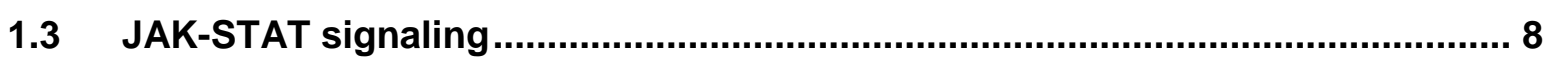

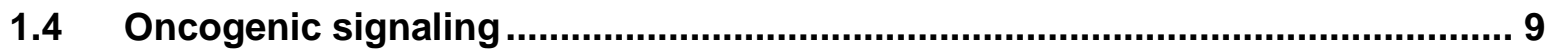

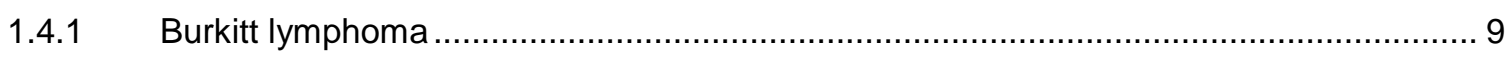

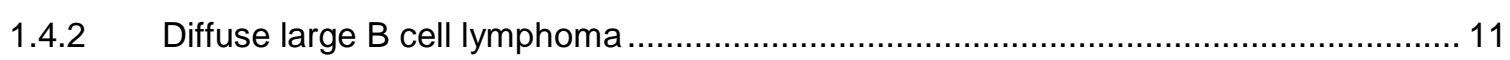

1.5 Important pathway interactions and crosstalks ........................................13

Aims

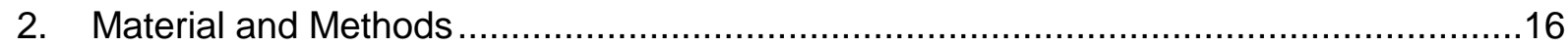

2.1 Material, recipes and equipment .............................................................16

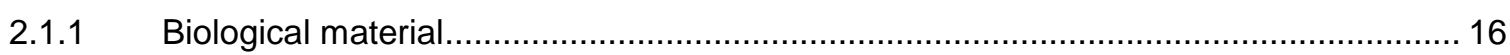

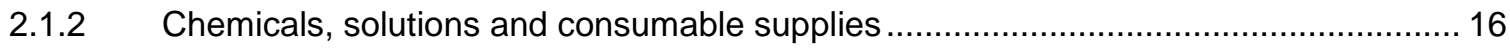

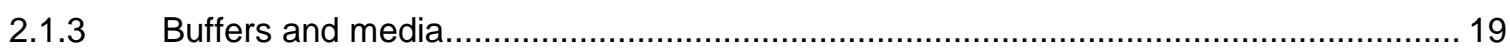

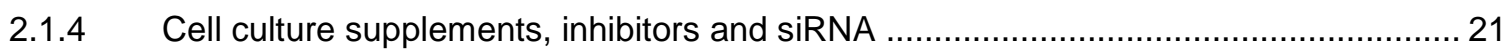

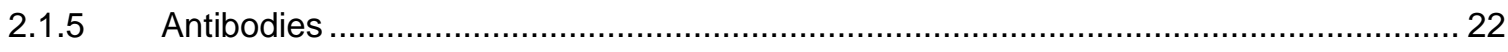

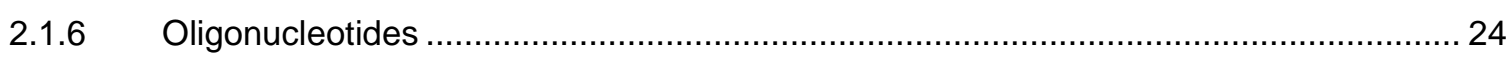

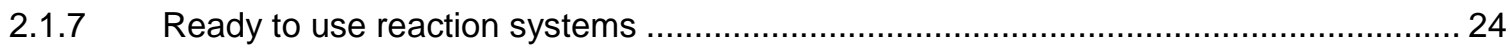

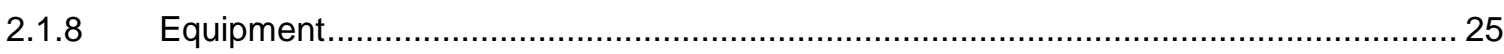

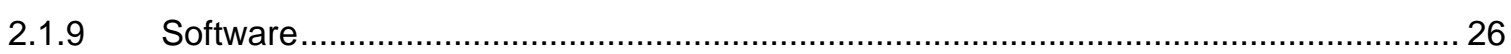

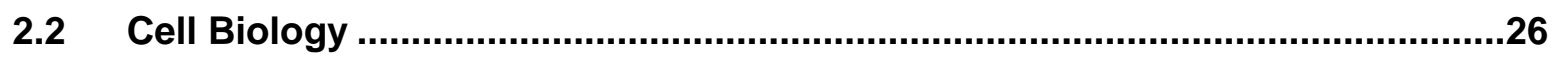

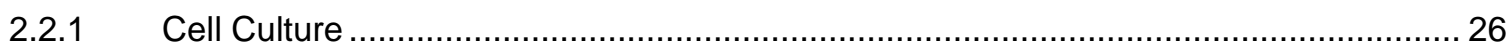

2.2.2 Stable isotope labeling by amino acids in cell culture .............................................. 27

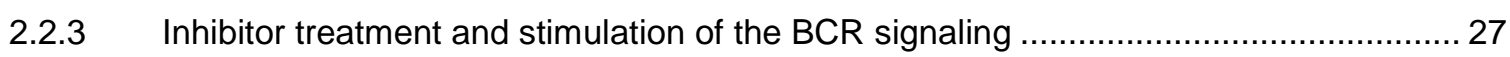




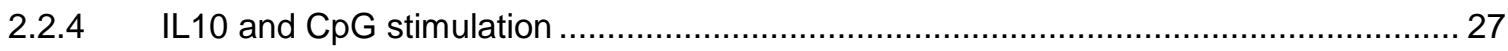

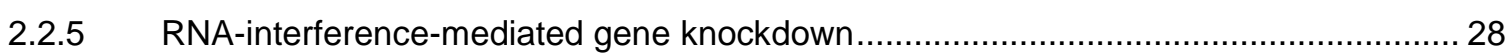

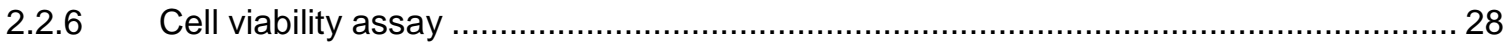

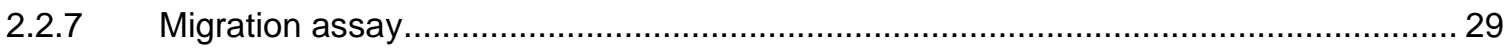

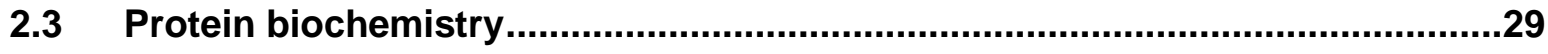

2.3.1 Preparation of cell lysates and cell fractionation for Western Blot analysis .................29

2.3.2 SDS Page, Western Blot and Immunodetection .............................................. 30

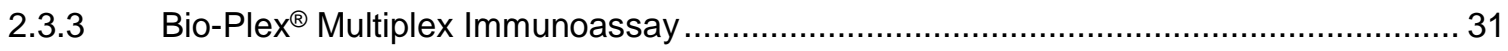

2.3.4 Mass spectrometry based phosphoproteomics ….............................................. 32

2.4 Molecular Biology .........................................................................................32

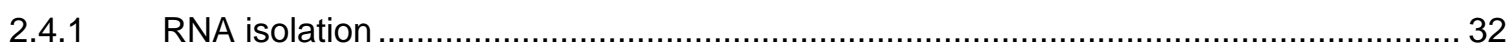

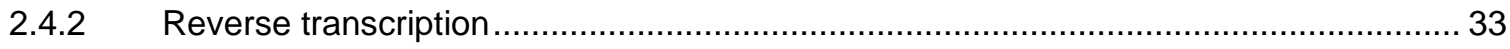

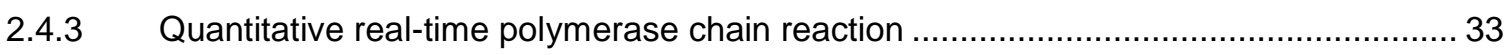

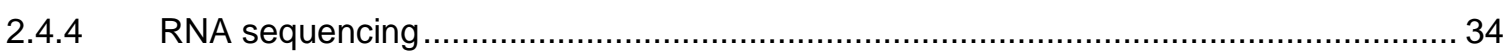

2.5 Statistics and bioinformatical analyses.......................................................35

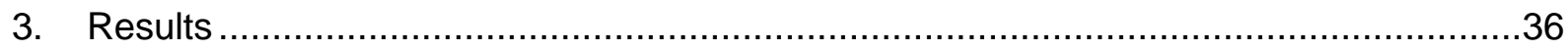

3.1 Tonic and active BCR signaling contains several feedback loops.....................36

3.1.1 Determination of a suitable time point for pathway interaction analyses .................... 37

3.1.2 Tonic BCR signaling is enhanced by positive feedback loop ..................................... 38

3.1.3 Active BCR signaling is influenced by positive and negative interplays..................... 40

3.1.4 Schematic model represents interactions of signaling pathways ............................ 43

3.2 p38 MAPK attenuates the MEK-ERK pathway ....................................................

3.2.1 p38a (MAPK14) limits the MEK-ERK pathway ................................................ 45

3.2.2 RAF-1 phosphorylations are affected by ERK1/2 but not by p38 MAPK ...................47

3.2.3 p38 MAPK reduces activation and duration of the MEK-ERK pathway .....................48

3.2.4 Early target gene expression of ERK1/2 is not influenced by p38 MAPK ...................50

3.2.5 p38 MAPK attenuates ERK1/2-regulated gene expression of the kinase MKK6 .........52

\subsection{DLBCLs with chronic active BCR contain divers feedback loops ....................56}

3.3.1 Positive and negative feedbacks influence chronic active BCR signaling ................... 56

3.3.2 Negative feedbacks of the chronic active BCR signaling have no impacts on

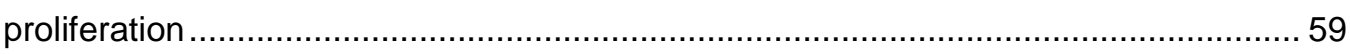

3.4 IL10R and TLR9 activation modify cell response and behavior......................60

3.4.1 IL10R and TLR9 activation change 239 protein phosphorylations ................................ 60

3.4.2 Phosphoproteome reveals influence on cell cycle, metabolism and migration ............. 63

3.4.3 Phosphoproteome analysis indicates MAP kinase involvement ................................. 65

3.4.4 IL10R and TLR9 activation reduce migration capacity of P493-6 cells .........................67 


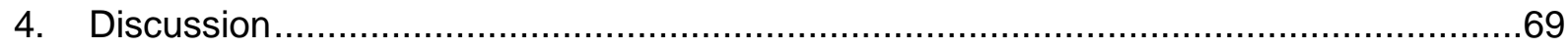

4.1 Positive feedback loop enhances tonic BCR signaling ..................................69

4.2 BCR signaling contains crosstalk from AKT and ERK1/2 to GSK3 $\beta \ldots . . . . . . . . . . .71$

4.3 MEK-ERK pathway is diminished by several negative feedbacks .....................73

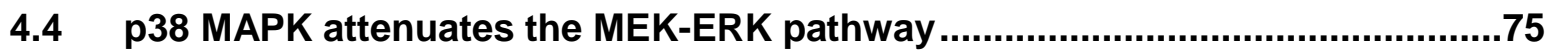

4.5 Feedback loops differ in ABC DLBCLs with chronic active BCR signaling.....77

4.6 Interplay of TLR9 and IL10R activation induces CDK2 and JNK......................79

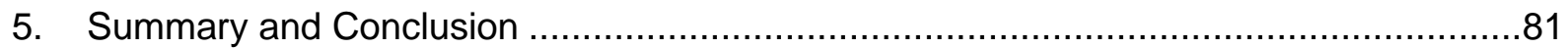

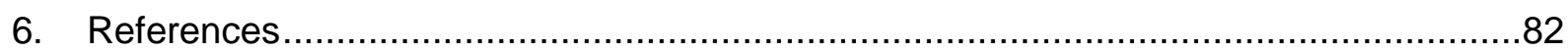

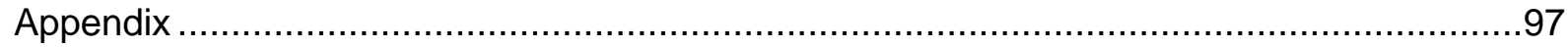

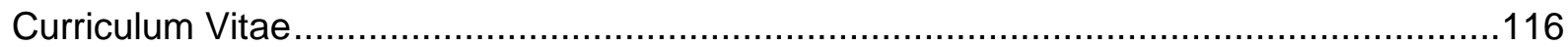




\section{Acknowledgment}

I would first like to thank my advisor Prof. Dieter Kube for his continuous support and supervision during the last years. I am grateful for his advice, helpful discussions and the opportunity to develop my own ideas.

I would sincerely like to thank Prof. Lorenz Trümper for the possibility to accomplish this thesis in his department.

In addition, I would like to express my gratitude to Prof. Heidi Hahn and Prof. Thomas Meyer for their guidance and encouragement during the thesis committee meetings.

I would also like to thank Bertram Klinger, Prof. Nils Blüthgen and Prof. Arnd Kieser for the great collaboration, the nice discussions and the lovely meetings. Furthermore, special thanks to Anja Sieber for the kind support with the multiplex immunoassay. I would like to thank Prof. Tim Beissbarth and Maren Sitte for the new calculation of the gene expression data. Moreover, I would like to acknowledge Prof. Henning Urlaub and Jasmin Corso for their support and the opportunity to perform the phosphoproteom analysis.

For always offering a helpful hand and their positive attitudes, a big thank you to Annekatrin Arlt and Franziska Linke. I would also like to thank Vanessa Kruse for her critical proofreading of this thesis, Frederike von Bonin and all former and present lab members for the great time.

Most importantly, I want to thank my family, my partner and my friends for their love, encouragement and unconditional support. I cannot thank my mum enough for being there for me and for all she has done for me. In addition, I would like to thank Matze for inspiring and supporting me. 


\section{Abstract}

The $B$ cell receptor $(B C R)$ signaling, required for the survival and maturation of $B$ cells, is one major deregulated pathway in B cell lymphomas. Several mutations are known to enhance the tonic BCR signal in Burkitt lymphomas (BL) or to mimic an activated receptor in some diffuse large $B$ cell lymphomas (DLBCL). While the proximal events and kinases of the BCR signaling are well studied, less is known about the interactions of downstream effector pathways. As the signaling interplays and feedback loops can influence the therapeutic success, this thesis aims for a better understanding of signaling interplays and for an improvement of oncogenic network models of B cell lymphomas.

For this purpose, BCR-related pathway interplays were examined by analyzing several protein phosphorylations with a multiplex immunoassay. Our investigations of pathway activations after thirteen defined perturbations revealed positive as well as negative interplays of pathways downstream of the BCR in BLs. During the tonic and active BCR signaling, the PI3K-AKT pathway, essential for many B cell lymphomas, enhanced its own activation probably through a positive feedback to kinases in close proximity of the BCR. We proposed that the positive feedback loop is one explanation for the potent effects of PI3K, AKT and mTOR inhibitors on BL proliferation. Furthermore, two negative feedbacks on the MEK-ERK pathway were detected after BCR activation. Beside ERK1/2 itself, p38 MAPK negatively influenced upstream kinases of ERK1/2. Interestingly, further fine-tuning of the p38 MAPK and ERK1/2 activation was assumed due to the ERK-mediated upregulation of MKK6 which could contribute to p38 MAPK activation. The mentioned feedbacks were generally identified downstream of the BCR signaling in all examined BL and DLBCL cell lines except for the p38 MAPK-dependent attenuation of the MEK-ERK pathway which was not observed in the DLBCL cell line OCI-LY3. In a second approach, the NF-KB and JAK-STAT-dependent proliferation of B cell lymphomas was investigated. Therefore, the signaling network following TLR9 and IL10R stimulation was analyzed by a phosphoproteom analysis. Our results revealed that the simultaneous activation of TLR9 and IL10R changed over 200 protein phosphorylations influencing cell cycle, metabolism and migration. The direct phosphorylation of CDK2 and JNK was suggested to contribute to the proliferative effect after TLR9 and IL10R activation.

The signaling interplays and feedbacks identified in this study provide a deeper insight and refinement of the signaling network structure in lymphomas. Additional improvements of computational network models are advantageous to predict signaling alterations by external influences as well as therapeutic responses. 


\section{List of Tables}

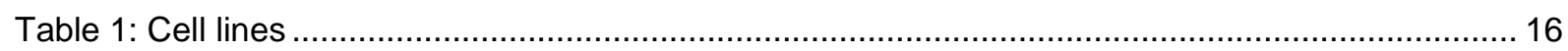

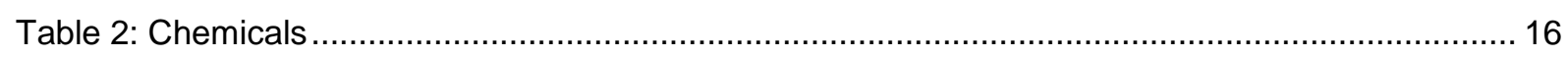

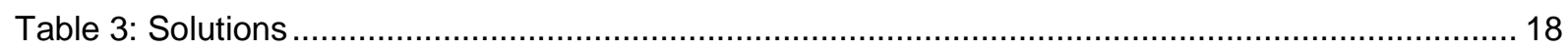

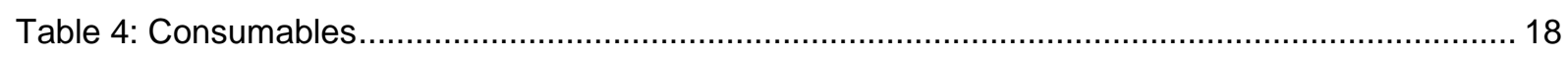

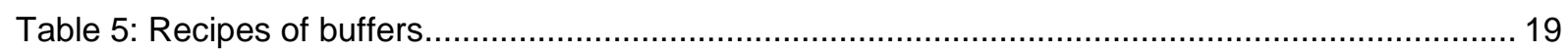

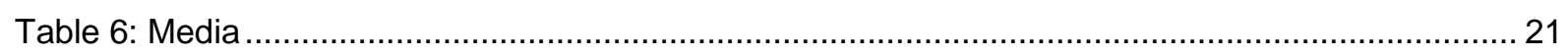

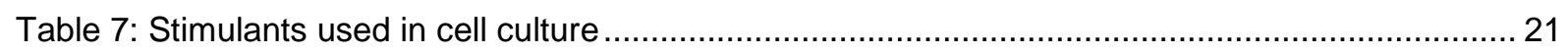

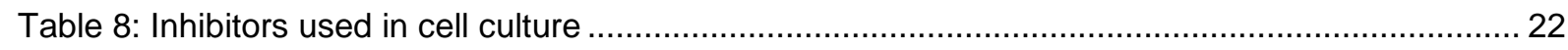

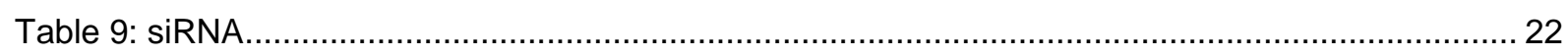

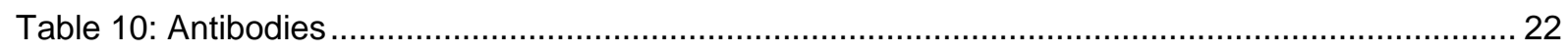

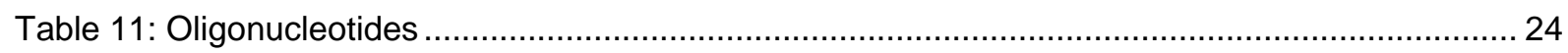

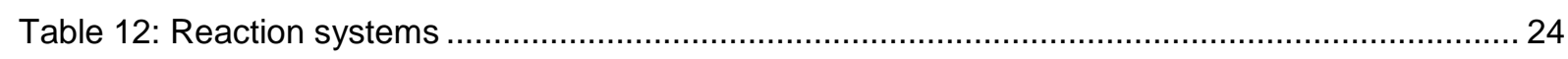

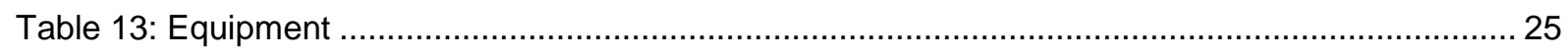

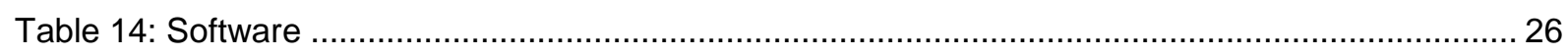

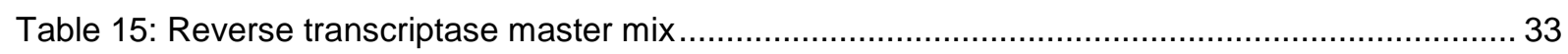

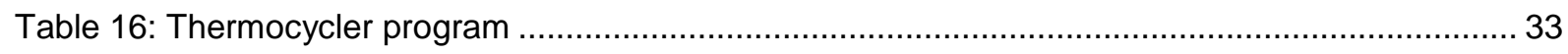

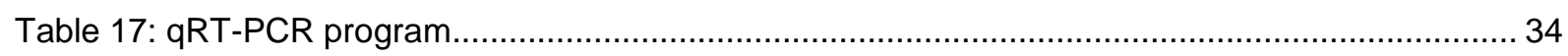

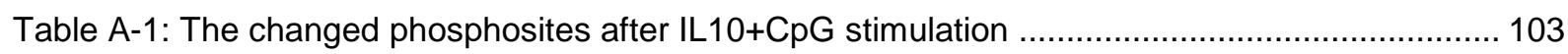

Table A-2: GO annotation of phosphoproteins changed by IL10+CpG stimulation ........................... 109 


\section{List of Figures}

Figure 1: Schematic representation of some known deregulated signaling pathways in BLs. 10

Figure 2: Schematic overview of some known deregulated signaling pathways in DLBCLs. 12

Figure 3: Time dependent activation of specific signaling pathways after BCR activation. 37

Figure 4: Signaling changes by pathway perturbations during the tonic BCR signaling. 39

Figure 5: Cell viability of BLs after pathway inhibition during the tonic BCR signaling. 40

Figure 6: Signaling changes by pathway perturbations during the active BCR signaling. 42

Figure 7: Network model of BCR signaling interactions and feedbacks. 44

Figure 8: Verification of TAK-1 and p38 MAPK influence on the MEK-ERK pathway ......................... 46

Figure 9: p38 MAPK and ERK1/2 impact on RAF-1 phosphorylations ............................................... 48

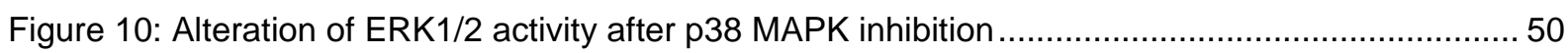

Figure 11: Impact on early target gene expressions by ERK1/2 and p38 MAPK .............................. 51

Figure 12: Impact of ERK1/2 and p38 MAPK on early target gene expression ...................................52

Figure 13: p38 MAPK influence on ERK1/2 target gene expression ............................................... 54

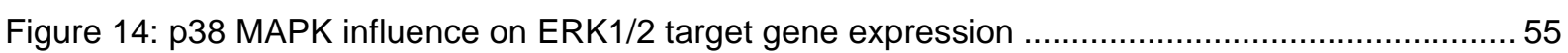

Figure 15: Signaling changes by pathway perturbations during the chronic active BCR signaling. ..... 57

Figure 16: Comparison of BL-2, HBL-1 and OCI-LY3 cells on protein and RNA level ........................59

Figure 17: Cell viability of HBL-1 and OCI-LY3 cells after MEK1/2 or p38 MAPK inhibition................. 60

Figure 18: Workflow and global phosphosites identification of mass spectrometry analysis ................ 61

Figure 19: Global phosphoproteomics results of Ctrl and IL10+CpG stimulated Myclow cells .............. 62

Figure 20: Network of affected phosphoproteins after IL10+CpG stimulation .................................... 63

Figure 21: GO annotation of biological process changed by IL10+CpG stimulation .......................... 65

Figure 22: IL10+CpG stimulation caused phosphorylation and expression of CDKs and MAPKs ....... 66

Figure 23: IL10+CpG impact on movement associated proteins and the migration potential ..............68

Figure 24: Proposed network scheme of pathway interplays for the tonic BCR signaling .................... 71

Figure 25: Proposed network scheme of pathway interplays for the activated BCR signaling ............. 74

Figure A-1: Multiplex immunoassay values and standard deviation of the BL-2 cell analysis ............. 98

Figure A-2: Multiplex immunoassay values and standard deviation of the BL-41 cell analysis ............ 99

Figure A-3: Literature-derived network model with the experimental setup ................................... 100

Figure A-4: Network model of BCR signaling interactions and feedbacks of BL-41 cells................... 101

Figure A-5: Multiplex immunoassay values and standard deviation of ABC DLBCLs analyses ......... 102

Figure A-6: GO annotation of phosphoproteins changed by IL10+CpG stimulation .......................... 115 


\section{Abbreviations}

$\begin{array}{ll}4 \text { E-BP1 } & \text { elF4E binding protein } \\ \alpha & \text { Anti } \\ \alpha-\operatorname{lgM} & \text { Anti-human IgM } F(a b ') 2 \\ \text { ABC } & \text { Activated B cell } \\ \text { AKT } & \text { v-akt murine thymoma viral oncogene homolog } \\ \text { APS } & \text { Adenosine phosphosulfate } \\ \text { ASK } & \text { Apoptosis signal regulating kinase } \\ \text { B2M } & \text { Beta 2 microglobulin } \\ \text { BAD } & \text { Bcl2-associated agonist of cell death } \\ \text { BAFF } & \text { B cell activating factor } \\ \text { BCAP } & \text { B cell adaptor for phosphatidylinositol 3 kinase } \\ \text { BCL6 } & \text { B cell lymphoma } 6 \text { protein } \\ \text { BCR } & \text { B cell receptor } \\ \text { BL } & \text { Burkitt lymphoma } \\ \text { BLNK } & \text { B cell linker } \\ \text { BSA } & \text { Albumin Fraction } V \\ \text { BTK } & \text { Bruton's tyrosine kinase }\end{array}$

CARD11

Caspase recruitment domain-containing protein 11

CD

Cluster of differentiation

CDK

Cyclin dependent kinase

CDKN2A

Cyclin dependent kinase inhibitor $2 \mathrm{~A}$

CDNA

Complementary DNA

Ctrl

Control

DAG

Diacyl glycerol

$\mathrm{dd}_{2} \mathrm{O}$

Double-distilled water

DEPC

Diethylpyrocarbonate

DLBCL

Diffuse large B cell lymphoma

DLK1

Protein delta homolog 1

DMSO

Dimethyl sulfoxide

DNA

Deoxyribonucleic acid

dNTP

Desoxyribonucleosid triphosphate

DPBS

Dulbecco's Phosphate Buffered Saline

DTT

Dithiothreitol

DUSP

Dual-specificity MAPK phosphatases

E2A

Transcription factor E2-alpha

EDTA

Ethylenediaminetetraacetic acid

EGR

Eearly growth response

EGTA

Ethylene glycol bis(2-aminoethyl ether) tetraacetic acid

ERK

Extracellular regulated kinase 
EZH2

FBS

FOXO

g

GAPDH

GCB

GNA13

GO

GRB2

GRB10

GSK3 $\beta$

GTP

HDAC1

HEPES

HIV

HLA

Hsp27

ID3

$\lg$

IKK

IL

IPBA

IRS-1

ITAM

JAK

JNK

$\mathrm{KCl}$

$\mathrm{kDa}$

KEA2

LCK

MALT1

MAP

MAPK

MAPKAPK

MEK

MEKK

$\mathrm{MgCl}_{2}$

MKK

MKP

MLK3

MTMR4
Enhancer of zeste homolog 2

Fetal Bovine Serum

Forkhead/winged helix box class $\mathrm{O}$

gravitational force

Glyceraldehyde-3-phosphate dehydrogenase

Germinal center B cell

G protein subunit alpha 13

Gene Ontology

Growth factor receptor-bound protein 2

Glycine-rich RNA-binding protein 10

Glycogen synthase kinase 3 beta

Guanosine triphosphate

Histone Deacetylase 1

4-(2-hydroxyethyl)-1-piperazineethanesulfonic acid

Immunodeficiency virus

Human leukocyte antigen

Small heat shock $27 \mathrm{kDa}$ protein

Inhibitor of DNA binding 3

Immunoglobulin

IKB kinase

Interleukins

lodophenylboronic acid

Insulin receptor substrate 1

Immunoreceptor tyrosine-based activation motifs

Janus kinase

c-JUN N-terminal kinase

Potassium chloride

Kilodalton

Kinase Enrichment Analysis 2

Lymphocyte cell-specific protein tyrosine kinase

Mucosa-associated lymphoid tissue lymphoma translocation protein 1

Mitogen-activated protein

Mitogen-activated protein kinase

Serine/threonine protein kinase MAPK-activated protein kinase

Mitogen-activated protein kinase kinase

Mitogen-activated protein kinase kinase kinase

Magnesium chloride

Mitogen-activated protein kinase kinase

MAP kinase phosphatases

Mixed linage kinase 3

Myotubularin-related protein 4 


\begin{tabular}{|c|c|}
\hline mTOR & Mammalian target of rapamycin \\
\hline mTORC & mTOR complex \\
\hline MYC & Myelocytomatosis oncogene cellular homolog \\
\hline Mychigh & P493-6 cells with $M Y C$ expression \\
\hline Myclow & P493-6 cells with reduced $M Y C$ expression \\
\hline MyD88 & Myeloid differentiation primary response 88 \\
\hline $\mathrm{NaCl}$ & Sodium chloride \\
\hline NFAT & Nuclear factor of activated T cells \\
\hline$N F-\kappa B$ & Nuclear factor kappa B \\
\hline NLRP11 & NACHT, LRR and PYD domains-containing protein 11 \\
\hline NOP2 & Nucleolar protein 2 homolog \\
\hline NOTCH2 & Neurogenic locus notch homolog protein 2 \\
\hline NP-40 & Nonylphenyl-polyethylene glycol \\
\hline$P$ & Phospho \\
\hline p90RSK & 90 kDa ribosomal protein S6 kinase \\
\hline PAGE & Polyacrylamide gel electrophoresis \\
\hline PAK & p21-activated kinase \\
\hline PALD1 & Paladin \\
\hline PARN & $\operatorname{Poly}(A)$-specific ribonuclease \\
\hline PDP1 & Pyruvate dehydrogenase phosphatase 1 \\
\hline PDK1 & Phosphoinositide-dependent kinase 1 \\
\hline PI3K & Phosphatidylinositol 3 kinase \\
\hline PIAS & Protein inhibitors of STATs \\
\hline PLCy2 & Phospholipase Cy2 \\
\hline PLD6 & Phospholipase D6 \\
\hline PMSF & Phenylmethanesulfonyl fluoride \\
\hline PRDM1 & PR/SET domain 1 \\
\hline PTEN & Phosphatase and tensin homolog \\
\hline PTGS1 & Prostaglandin-endoperoxide synthase 1 \\
\hline PTPN & Tyrosine protein phosphatase non-receptor type \\
\hline qRT-PCR & Quantitative real-time polymerase chain reaction \\
\hline RAS & Rat sarcoma \\
\hline RASGRP1 & RAS guanyl-releasing protein 1 \\
\hline RCSD1 & CapZ-interacting protein 1 \\
\hline RNA & Ribonucleic acid \\
\hline RPS6 & $40 S$ ribosomal protein S6 \\
\hline RT & Room temperature \\
\hline $\mathrm{S}$ & Serine \\
\hline S6K & Ribosomal S6 kinase \\
\hline SATB1 & Special AT-rich sequence-binding protein 1 \\
\hline scrb & Negative control \\
\hline
\end{tabular}




$\begin{array}{ll}\text { SDS } & \text { Sodium dodecyl sulfate } \\ \text { SFK } & \text { SRC family tyrosine kinases } \\ \text { SH2 } & \text { Src Homology } 2 \\ \text { SHP } & \text { SH2 domain containing protein tyrosine phosphatase } \\ \text { SILAC } & \text { Stable isotope labeling by amino acids in cell culture } \\ \text { siRNA } & \text { Small interfering RNA } \\ \text { SOS } & \text { Son of sevenless homolog } \\ \text { SOCS } & \text { Suppressors of cytokine signaling } \\ \text { STAT } & \text { Signal transducer and activator of transcription } \\ \text { SYK } & \text { Spleen tyrosine kinase } \\ \text { T } & \text { Threonine } \\ \text { TAK1 } & \text { TGF-beta activated kinase 1 } \\ \text { TBS } & \text { Tris buffered saline } \\ \text { TEMED } & \text { Tetramethylethylenediamine } \\ \text { TLR } & \text { Toll like receptors } \\ \text { TRAF6 } & \text { TNF receptor-associated factor 6 } \\ \text { Tris HCl } & \text { Tris(hydroxymethyl)-aminomethanhydrochlorid } \\ \text { v/v } & \text { volume/volume } \\ \text { w/v } & \text { weight/volume } \\ \text { Y } & \text { Tyrosine } \\ \text { ZAP70 } & \text { 70 kDa zeta-chain associated protein } \\ \text { ZFP36L1 } & \text { Zinc finger protein 36 C3H1 type-like 1 } \\ \text { ZNF800 } & \text { Zinc finger protein 800 }\end{array}$

\section{Deoxyribonucleotides}

$\begin{array}{ll}\text { G } & \text { deoxyguanosine monophosphate } \\ \text { A } & \text { deoxyadenosine monophosphate } \\ T & \text { deoxythymidine monophosphate } \\ \text { C } & \text { deoxycytidine monophosphate }\end{array}$

Units and Prefixes are in conformity with the International system of Units. 


\section{Introduction}

The immune system consists of various cell types to counteract pathogens through recognition, destruction and disposal. B cells including antibody secreting plasma cells and memory $B$ cells are involved in the detection and labeling of foreign molecules. To cover the diversity and to increase the affinity of antibodies, B cells have the opportunity to modify their immunoglobulins. The adaptation and selection of B cells secreting highaffinity antibodies occur in germinal centers (Klein and Dalla-Favera, 2008; Natkunam, 2007). Germinal centers are histological structures in lymphoid tissues with a dark and a light zone (MacLennan, 1994). In the dark zone, B cells proliferate extensively and undergo somatic hypermutations to alter their antigen specificity. To further adjust the effector functions, B cells perform class switch recombination in the light zone. Depending on the suitability of the antibody affinity, B cells are selected in the light zone to exit the germinal center. All other B cells with unsuitable antibody affinity die by apoptosis or re-enter the dark zone for further somatic hypermutations (Victora and Nussenzweig, 2012; Victora et al., 2010).

The adaptation and selection of antibody secreting B cells follow a distinct program of signals and transcriptional alterations. Beside the activation of B cells, cell-cell interactions and costimulatory signals are essential to induce a specific set of intracellular signaling cascades and transcriptional modulators. These pathway activations regulate $\mathrm{B}$ cell proliferation, somatic hypermutations or class switch recombination during the germinal center reaction (Kuppers, 2005; Pone et al., 2010). Although different check points serve as a strong control to avoid and eliminate mistakes, the gene remodeling process provides an opportunity for B cells to transform into malignancies like B cell lymphomas (Basso and Dalla-Favera, 2015; Victora et al., 2012).

\subsection{Non-Hodgkin Lymphoma}

B cell lymphomas comprise a wide spectrum of malignancies and thus are further categorized in Hodgkin and non-Hodgkin lymphoma due to different genetically, phenotypically and clinically aspects (Vardiman et al., 2009). B cell lymphomas derive from mature B cells during the germinal center reaction (Kuppers, 2005). The heterogeneity of lymphomas reflects the origin from distinct phases of the germinal center reaction. Therefore, lymphomas can be classified by the $B$ cell state of origin and on the basis of genetic alterations causing abnormal signal transductions (Victora et al., 2012). The aggressive non-Hodgkin lymphomas include Burkitt lymphoma (BL) and diffuse large 
B cell lymphoma (DLBCL). While BLs derive from germinal center B cells of the dark zone, the DLBCLs are closer related to B cells from the light zone (germinal center B cell like) or to early stages of post germinal center plasma cell differentiation (activated B cell like) (Basso and Dalla-Favera, 2015).

\subsubsection{Burkitt Lymphoma}

BL was first described by Denis Burkitt as a highly aggressive B cell lymphoma in African children (Burkitt, 1958). The characteristics of BLs are uniform, highly proliferating cells and a starry-sky appearance due to tingible body macrophages phagocytosing the apoptotic debris (Blum et al., 2004). Nowadays, BLs are subdivided in three different types with some distinct pathological features (Molyneux et al., 2012). The endemic variant occurs predominantly in 4 to 7 years old children of equatorial Africa. A variant affecting children and young adults worldwide is classified as sporadic BL. The third, the immunodeficiency-related type is associated with immunodeficiency virus (HIV) infected individuals or with posttransplant complications. In addition, an infection with the EpsteinBarr virus is related to all endemic, $15 \%$ of sporadic and $40-50 \%$ of immunodeficiencyassociated BLs although its impacts are still discussed (Molyneux et al., 2012; Spender and Inman, 2014). BLs account for 40 - $50 \%$ of lymphomas in children and only $1-2 \%$ of lymphoma cases in adults (Aldoss et al., 2008).

The typical immune-phenotype of BLs comprises high Ki67 indices, the expression of surface immuno-globulin $\mathrm{M}(\mathrm{IgM})$ and $\mathrm{B}$ cell markers such as cluster of differentiation (CD) 10, CD19, CD20, CD22 (Kelemen et al., 2010). Furthermore, BLs are characterized by a chromosomal translocation affecting the proto-oncogene myelocytomatosis oncogene cellular homolog $(M Y C)$. The translocation places MYC under the control of the regulatory elements of $\mathrm{lg}$ and thus causes an aberrant expression. The transcription factor c-MYC is involved in regulation of proliferation, cell growth, differentiation, metabolism and apoptosis (Basso and Dalla-Favera, 2015). In addition, the transcription factor E2-alpha (E2A) or its negative regulator inhibitor of DNA binding 3 (ID3) are frequently mutated in $70 \%$ of $\mathrm{BL}$ patients and $38 \%$ of $\mathrm{BL}$ patients have cyclin D3 aberrations (Richter et al., 2012; Schmitz et al., 2012). E2A is known to enhance phosphatidylinositol 3-kinase (PI3K) activation and constitutive PI3K and c-MYC activation is sufficient to provoke BL-like lymphomas in transgenic mice. However, due to a clonality of the tumor, further mutations are suggested to be necessary for lymphomagenesis (Klapproth and Wirth, 2010; Sander et al., 2012; Schmitz et al., 2012). 


\subsubsection{Diffuse Large B cell Lymphoma}

DLBCLs are the most frequent type of non-Hodgkin lymphomas in adults accounting for $40 \%$ of cases. The medium to large-sized lymphoid cells are arranged in a diffuse pattern and displacing the normal tissue architecture (Boyd et al., 2013). Due to the molecular diversity and different clinical outcomes, DLBCLs can be distinguished by different criteria. In Germany, DLBCLs are subdivided by their centroblastic or immunoblastic morphology (Stein and Hummel, 2006). Furthermore, a molecular classification was introduced by Alizadeh and coworkers. The germinal center $B$ cell (GCB) like and activated $B$ cell (ABC) like DLBCLs are characterized by subtype specific mutations although some genetic alterations are found in both subtypes (Alizadeh et al., 2000). A common feature of all DLBCLs is the influence on chromatin modifiers and the immune escape (Basso and Dalla-Favera, 2015).

The typical immune-phenotype of DLBCLs comprises the expression of the surface immunoglobulin $M(\lg M)$ or $G(\lg G)$ and B cell markers such as CD19, CD20, CD22 and CD79a (Boyd et al., 2013). Common genetic alterations of DLBCLs increase the activity of B cell lymphoma 6 protein (BCL6) in order to enhance proliferation, to suppress DNA damage response and to block terminal differentiation (Basso and Dalla-Favera, 2015). Additionally, defects of cell surface markers promote the immune escape. Mutations of human leukocyte antigen class I (HLA-I), beta 2 microglobulin (B2M) and CD58 cause invisibility to cytotoxic T cells and nature killer cells (Challa-Malladi et al., 2011). Apart from these common features, the pathogenesis of GCB DLBCLs is poorly understood and only in a fraction of cases mutations of phosphatase and tensin homolog (PTEN), BCL2, c-MYC, enhancer of zeste homolog 2 (EZH2) or G protein subunit alpha 13 (Ga13) are found (Basso and Dalla-Favera, 2015; Rickert, 2013). Aberrations of EZH2 enhance proliferation, impair differentiation and promote germinal center formation. However, mutations of EZH2 are insufficient to induce DLBCL development and additional alterations of for example BCL2 are required to accelerate lymphomagenesis in mice (Beguelin et al., 2013). On the contrary, ABC DLBCLs depend on constitutive active nuclear factor kappa B (NF-kB) which is induced for instance by oncogenic mutations of BCR components, myeloid differentiation primary response 88 (MyD88) or caspase recruitment domain-containing protein 11 (CARD11) (Davis et al., 2001; Young and Staudt, 2013). The blockage of terminal differentiation in ABC DLBCLs can occur through inactivation of PR/SET domain 1 (PRDM1) (Pasqualucci et al., 2006). Interestingly, $P R D M 1$ inactivation promotes lymphoma development in mice with critical features of ABC DLBCLs (Mandelbaum et al., 2010). 


\subsection{BCR signaling}

The PI3K and NF-KB pathways, aberrantly regulated in BLs and DLBCLs, are major components of the BCR signaling. Many non-Hodgkin lymphomas show a strong dependency on the BCR signaling implicating a pivotal role of the BCR in lymphomagenesis (Young and Staudt, 2013). The BCR is essential for normal B cell development and maturation. The strength of BCR signaling and the additional activation of cofactors like CD40, toll-like receptors (TLR) and the survival factor B cell activating factor (BAFF) are necessary for the adaptation and survival of the B cells during the germinal center reaction (Pone et al., 2010). While the survival of resting mature B cells depends on a basic so called "tonic" BCR signal, the activation of the BCR by antigens induces B cell proliferation, maturation and antibody production (Avalos and Ploegh, 2014; Lam et al., 1997).

A functional BCR consists of a membrane bound immunoglobulin and the two co-receptors Ig alpha (CD79a) and Ig beta (CD79b) (Kurosaki, 1999). In normal and malignant Bcells, the BCR can transmit a tonic signal through the PI3K pathway or induces further pathway activations after recognition of an antigen (Rickert, 2013). Ligation of an antigen to the BCR induces conformational changes and crosslinking of several receptors (Avalos and Ploegh, 2014). Hence, tyrosine phosphorylations within the immunoreceptor tyrosine-based activation motifs (ITAMs) of CD79a and CD79b are triggered by SRC family tyrosine kinases (SFKs) such as Lyn. These phosphorylations promote the binding of spleen tyrosine kinase (SYK) and initiate the formation of a signalosome complex (Jin et al., 2013; Kurosaki, 1999; Pao et al., 1998). The complex is assembled by adaptor proteins and multiple tyrosine kinases. While adaptor proteins as $B$ cell linker (BLNK) and B cell adaptor for phosphatidylinositol 3-kinase (BCAP) manage the signal distribution, tyrosine kinases like Bruton's tyrosine kinase (BTK) and phospholipase Cy2 (PLCY2) forward the signal (Jin et al., 2013; Takata and Kurosaki, 1996; Wienands et al., 1998). Besides, phosphatases are also implicated in the BCR signaling regulation. The $\mathrm{SH} 2$ domain-containing protein tyrosine phosphatase 1 (SHP-1) counteracts the activity of SRC and SYK whereas the SH2 domain-containing protein tyrosine phosphatase 2 (SHP2) enhances the signaling especially of the extracellular regulated kinase (ERK) pathway (Jiang et al., 2014; Pao et al., 2007; Tartaglia et al., 2004).

Although even further signaling events occur, the outcome of proximal BCR signaling leads to activation of the NF-KB, PI3K, mitogen-activated protein kinase (MAPK) and nuclear factor of activated T cells (NFAT) pathway (Kurosaki, 2011; Niiro and Clark, 2002). The signal transmission through the MAPK pathways includes ERK, p38 MAPK 
and JUN N-terminal kinase (JNK) activation (Jiang et al., 1998). All BCR-induced pathway activations contribute to proliferation and survival of $B$ cells with the exception of the NFAT pathway. The NFAT pathway is suggested to modulate B cell responses in plasma cell differentiation (Niiro and Clark, 2002; Winslow et al., 2006). In the following sections, the pathways relevant for this study are described in more detail.

\subsubsection{PI3K-AKT-mTOR pathway}

Activation of the PI3K pathway is sufficient to maintain resting mature B cell survival after depletion of the BCR. Therefore, the PI3K signal transduction is suggested to be the main component of the tonic BCR signaling (Srinivasan et al., 2009). Besides, the tonic signal is essential for many B cell malignancies although the PI3K itself is infrequently mutated (Blachly and Baiocchi, 2014).

The BCR signal is transmitted by the adapter protein BCAP to a PI3K heterodimer consisting of a catalytic and a regulatory subunit (Blachly and Baiocchi, 2014; Okada et al., 2000). A variety of effectors are further involved. On the one hand, PI3K together with BTK activates NF-kB. On the other hand, v-akt murine thymoma viral oncogene homolog (AKT) acts as an immediate effector between PI3K and mammalian target of rapamycin (mTOR). For this purpose, PI3K induces phosphoinositide-dependent kinase-1 (PDK1) and along with the mTOR complex 2 (mTORC2) the serine-threonine protein kinase AKT is recruited and phosphorylated. Following, AKT phosphorylates the mTOR complex 1 (mTORC1) and additionally inactivates Forkhead/winged helix box class $O$ (FOXO) to induce cell cycle progression and survival (Baracho et al., 2011; Brunet et al., 1999; Rickert, 2013). Two of the best characterized downstream targets of mTORC1 are the ribosomal S6 kinase (S6K) and the elF4E binding protein (4E-BP1) (Laplante and Sabatini, 2012). The signaling affects protein synthesis, nutrient response and many additional functions required for a rapid growth (Limon and Fruman, 2012). Furthermore, a S6K-dependent inactivation of insulin receptor substrate 1 (IRS-1), S6K-dependent suppression of mTORC2 and a mTORC1-dependent phosphorylation of the adaptor protein glycine-rich RNA-binding protein 10 (GRB10) serve as negative feedback mechanisms to reduce the upstream PI3K-AKT signaling (Logue and Morrison, 2012; Tremblay et al., 2007; Yea and Fruman, 2011).

\subsubsection{MEK-ERK pathway}

Several MAPK pathways like ERK are activated following BCR activation and transmit the signal through a distinct core cascade of kinases (Dhillon et al., 2007). ERK plays an important role in B cell development and is required for the proliferative expansion of 
immature and the differentiation of mature B cells (Yasuda et al., 2011; Yasuda et al., 2008). Similar to other cancer identities, constitutive ERK activation is also described for B cell malignancies (Ogasawara et al., 2003).

For initiation, receptor tyrosine kinases trigger the loading of guanosine triphosphate (GTP) to the small GTPase rat sarcoma (Ras). After BCR activation this is mediated rather by the guanine nucleotide exchange factor RAS guanyl-releasing protein 1 (RASGRP1) then by the growth factor receptor-bound protein 2 (GRB2)- son of sevenless homolog (SOS) complex (Oh-hora et al., 2003). Once activated the GTP-bound Ras recruits RAF family members like RAF-1 and B-RAF to the plasma membrane for activation. The effector protein mitogen-activated protein kinase kinase 1 and 2 (MEK1/2) is activated by RAF and phosphorylates ERK1 and ERK2 (ERK1/2) on a threonine-Xtyrosine motif (Alessi et al., 1994; Dhillon et al., 2007; Wellbrock et al., 2004).

Phosphorylated ERKs can function in the cytosol or dimerize and translocate to the nucleus (Chen et al., 1992). In the nucleus, ERK1/2 contributes to several tasks which are determined by the signal strength and duration. Early target gene expressions and activations of the proto-oncogene FOS, early growth response 1 (EGR-1), MYC or protooncogene JUN point to a sustained signaling of ERK1/2 (Dhillon et al., 2007; Murphy and Blenis, 2006). Furthermore, about hundred other targets are modulated in an ERKdependent manner (Roskoski, 2012). Interestingly, upstream pathway components are affected by ERK1/2 target genes indicating the existence of autoregulatory feedback loops (Corbalan-Garcia et al., 1996; Dougherty et al., 2005). For instance, ERK1/2 induces the expression of Sprouty to prevent the GRB2-SOS complex and MAP kinase phosphatases (MKPs) to reduce its own activation (Hanafusa et al., 2002; Ozaki et al., 2001; Sun et al., 1993). Additionally, the ERK-dependent phosphorylations of SOS, RAF and MEK1 lead to inactive conformations, reduced binding capacities and finally to a decreased pathway activation (Brunet et al., 1994; Dhillon et al., 2007).

\subsection{3 p38 MAPK pathway}

The p38 MAPK pathway is also activated by a distinct kinase cascade and is induced by inflammatory cytokines and environmental stress factors. However, the role and impacts of p38 MAPK are versatile and still controversially discussed (Ding et al., 2009; Trempolec et al., 2013a). Nevertheless, p38 MAPK activation is found in numerous B cell malignancies and predicts the failure of response in CHOP-treated DLBCL patients (Vega et al., 2015). 
The first kinases of the p38 MAPK activating cascade are mitogen-activated protein kinase kinase kinase 3 and 4 (MEKK3/4), apoptosis signal regulating kinase (ASK), protein delta homolog 1 (DLK1), mixed linage kinase 3 (MLK3) and TGF-beta activated kinase 1 (TAK1). After activation of one of the first kinases, mitogen-activated protein kinase kinase 3 (MKK3), 4 (MKK4) and 6 (MKK6) are induced to further activate one out of five p38 MAPK subunits. Thereby, a threonine-X-tyrosine motif existing in all subunits is phosphorylated (Feng et al., 2009; Zarubin and Han, 2005).

In the cytosol, p38 MAPK regulates protein activations, for instance, the activation of the serine/threonine protein kinase MAPK-activated protein kinase 2 (MAPKAP-K2) with subsequent small heat shock $27 \mathrm{kDa}$ protein (Hsp27) activation. Furthermore, p38 MAPK initiates protein degradation through phosphorylation-mediated destabilization or ligase activation (Cuenda and Rousseau, 2007; Trempolec et al., 2013a). After activation p38 MAPK can also translocate into the nucleus to enhance transcription factor activity for a rapid induction of immediate-early genes. The p38 MAPKs are emerging as important modulators of gene expression by regulation of chromatin modifiers and remodelers (Ashwell, 2006; Trempolec et al., 2013b; Zarubin and Han, 2005). Several targets and anti-apoptotic as well as pro-apoptotic functions are described and so far not elucidated in detail (Feng et al., 2009).

\subsubsection{NF-kB pathway}

The NF-kB pathway is important for the proliferation and survival of $B$ cells as it counteracts apoptotic signals (Jost and Ruland, 2007). An aberrant NF-kB activation is a hallmark of several B cell malignancies to induce cell cycling and to block apoptosis (Staudt, 2010).

Through PLCY2, BTK and the adapter protein BLNK the canonical NF-KB pathway activation is initiated (Niiro and Clark, 2002). In addition to diacyl glycerol (DAG) and increased intracellular calcium flux, PLCY2 activity induces the classical isoform PKC $\beta$ to phosphorylate CARD11 (Young and Staudt, 2013). Furthermore, the mucosa-associated lymphoid tissue lymphoma translocation protein 1 (MALT1) associates with BCL-10 and becomes activated. After multimerization with CARD11, TNF receptor-associated factor 6 (TRAF6) and TAK1 are recruited to the complex. TRAF6 further activates IKB-kinase (IKK) in an ubiquitin-dependent manner while TAK1 leads to phosphorylation of the activation loop of IKK. IKK-mediated phosphorylations of the inhibitory protein IKB triggers IKB polyubiquitinylation with subsequent proteolytic degradation thus releasing the transcription factor NF-KB (Ruland and Mak, 2003; Shinohara et al., 2005; Staudt, 2010; Thome et al., 2010). Besides, the alternative NF-KB pathway also activates IKKs to induce 
a direct phosphorylation and partial proteolysis of specific subunits of NF-KB (Jost and Ruland, 2007).

As result heterodimers are formed consisting the subunits RelA (p65), RelB, c-Rel, NF-kB1 (p50) and NF-kB2 (p52). The heterodimeric NF-kB transcription factors accumulate in the nucleus and activate the transcription of target genes (Jost and Ruland, 2007). NF-kB target genes include positive cell cycle regulators, anti-apoptotic proteins, inflammatory and immunoregulatory factors as well as negative feedback regulators to decrease the activation of upstream pathway components. For instance, cell cycle regulators like $M Y C$ and cyclin D1 are upregulated and anti-apoptotic proteins of the $\mathrm{BCL}-2$ family are enhanced by NF-KB. The expression of immunoregulatory cytokines includes interleukins (IL) like IL2, IL6 and IL10 to activate growth receptors in an autocrine or paracrine fashion (Jost and Ruland, 2007; Lam et al., 2008).

\subsection{JAK-STAT signaling}

Autocrine as well as paracrine secreted interleukins are the main activators of the Janus kinase (JAK) and signal transducer and activator of transcription (STAT) pathway (Leonard and Lin, 2000). The JAK-STAT pathway is a pivotal signaling to regulate cell proliferation, survival, differentiation and immune response (Levy and Darnell, 2002). In addition to BCR signaling, several interleukin serum levels are elevated in non-Hodgkin lymphomas (Fabre-Guillevin et al., 2006). Especially in ABC DLBCLs the aberrant regulated NF-KB pathway leads to the expression of IL6 or IL10 and thereby to a subsequent growth factor stimulation (Gupta et al., 2012; Jost and Ruland, 2007).

The binding of a cytokine to a basally inactivated cognate receptor initiates to conformational changes or dimerization of the receptor. Intracellular bound JAKs become activated and trigger cross phosphorylations with the receptor. The phosphorylation sites serve as anchor points for STAT proteins to receive phosphorylations through JAKs (Ihle, 1995). For instance, in non-Hodgkin lymphoma the receptor activation by IL6 or IL10 causes a signal transmission over JAKs to STAT3 (Gupta et al., 2012; Lam et al., 2008). Besides, JAKs can activate the MAPK, PI3K and mTOR pathway while receptor internalization or phosphatase recruitment terminates the JAK activity (Vainchenker and Constantinescu, 2013).

Phosphorylated STATs can dimerize and translocate into the nucleus where they perform their task as transcription factors. Apart from proliferation and survival associated genes, STATs regulate the transcription of suppressors of cytokine signaling (SOCS) (Rawlings 
et al., 2004). SOCS 1 to 7 belong to negative feedback loops of this pathways and deactivate STAT signaling by direct binding to JAK and hence preventing further STAT phosphorylation. In addition, protein inhibitors of STATs (PIAS) interact directly with STATs to repress the transcriptional activity (Vainchenker and Constantinescu, 2013).

\subsection{Oncogenic signaling}

The complexity of the germinal center reaction provides many vulnerabilities for B cells to transform into malignancies. Mutations, leading to aberrant signaling through modulation of cascades and feedbacks, play an important role in tumor progression and survival (Basso and Dalla-Favera, 2015; Young and Staudt, 2013). Due to the heterogeneity of lymphomas and their causing mutations, the understanding of molecular pathways that drive and maintain tumorigenesis is necessary in order to improve therapies and to avoid resistances or relapses (Schmitz et al., 2012; Victora et al., 2012). Despite subtype specific mutations and different clinical outcomes, many B cell malignancies are highly sensitive to kinase inhibitors disrupting the BCR signaling. Thus, targeted therapy aiming at the BCR signaling emerges as a new treatment opportunity for several B cell malignancies (Smith, 2015).

\subsubsection{Burkitt lymphoma}

BLs in young patients are often cured by high dose chemotherapy whereas the outcome for elderly patients is worse due to therapeutic intolerance. The common chemotherapy of BLs includes DNA and cell cycle damaging reagents beside glucocorticoids to target cells with high proliferation rates. The treatment-associated immune suppression is a major hurdle in less developed regions emphasizing the need for new targetable candidates (Aldoss et al., 2008; Schmitz et al., 2012).

In BLs the genetic aberrations apart from c-MYC are often associated with an enhanced PI3K pathway comprising the tonic BCR signal (Figure 1) (Spender and Inman, 2014). E2A and its negative regulator ID3 are normally expressed in germinal center B cells of the dark zone to modulate the BCR signaling (Ott et al., 2013). In BLs ID3 is often mutated as well as the ID3 binding site in E2A. As a result, E2A activation is enhanced and appears to promote the PI3K signaling by inhibiting the BCR-related phosphatase SHP-1 (Love et al., 2012; Schmitz et al., 2014). Another mechanism to induce the PI3K-AKT pathway is the modulation of the negative regulator PTEN. While direct PTEN mutations are infrequent, the upregulation of the miR-17-92 cluster occurs more often in order to inhibit PTEN expression in BLs (Lenz et al., 2008c; Schmitz et al., 2012). In 
addition to the tonic signal promotion, E2A could also induce the cell cycle through its downstream target cyclin D3 (CCND3). Cyclin D3 induces cell cycle progression along with cyclin dependent kinase 6 (CDK6). Furthermore, repression of the CDK6 inhibitor cyclin dependent kinase inhibitor $2 A(C D K N 2 A)$ is a common lesion in BLs to promote proliferation (Schmitz et al., 2012; Spender and Inman, 2014).

As many mutations in $\mathrm{BL}$ enhance the tonic $\mathrm{BCR}$ signaling, the PI3K pathway is suggested as a new druggable pathway for the treatment of BL. The inhibition of PI3K is so far approved for chronic lymphocytic leukemia and indolent lymphoma (Smith, 2015). In vitro studies using $\mathrm{BL}$ cell lines clearly demonstrated that proliferation is reduced after inhibition of PI3K, AKT, mTOR and cyclin D3/CDK6 (Spender and Inman, 2014). Therefore, the number of small pharmaceutical molecules targeting BCR-specific kinases increases steadily but their benefits still remains to be examined in clinical trials (Smith, 2015).

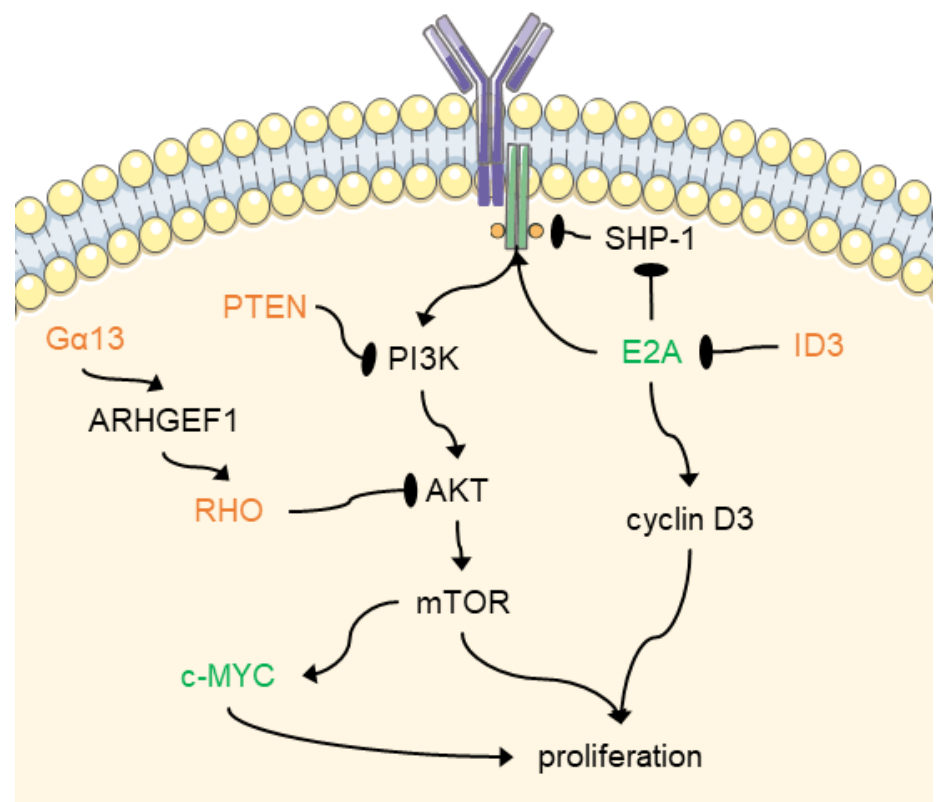

Figure 1: Schematic representation of some known deregulated signaling pathways in BLs.

Activation of C-MYC and the PI3K pathway are the main oncogenic alterations in BLs. An enhanced activation of E2A or a missing suppression of PI3K by PTEN contributes to the tonic BCR signaling in BLs. Black lines represent the interaction. Upregulated and downregulated proteins are displayed in green and red, respectively. Adapted from Spender and Basso (Basso and DallaFavera, 2015; Spender and Inman, 2014). 


\subsubsection{Diffuse large B cell lymphoma}

Due to their divergent genetic mutations and the different developmental states they derive from, DLBCL subtypes have various pathway dependencies. GCB DLBCLs mainly comprise a tonic BCR signaling and occur more often in younger patients. Mutations in $A B C$ DLBCLs mimic a chronic active BCR with constitutive activation of the NF-KB pathway and are associated with worse outcomes than GCB DLBCLs. However, GCB and ABC DLBCLs cannot be clearly distinguished and both subtypes are treated with the same standard therapy (Deeb et al., 2015; Lenz et al., 2008b; Pfeifer and Lenz, 2013). As $40 \%$ of all DLBCL cases are still incurable, a better understanding of deregulated pathways in each subtype is needed to reveal similarities as well as differences (Ott et al., 2010).

The pathogenesis of GCB DLBCLs is poorly understood, however some aberrations are quite similar to BLs (Figure $2 \mathrm{~A}$ ). In a fraction of cases mutations of PTEN, c-MYC, BCL-2, EZH2 or Ga13 are found (Basso and Dalla-Favera, 2015; Rickert, 2013). Similar to BLs, $\mathrm{c}-\mathrm{MYC}$ enhances cell cycle progression but this is rather mediated by gain of low gene copy numbers or mutations of C-MYC regulators than by genetic translocations as seen in BLs (Ott et al., 2013). Furthermore, like in BLs the PI3K-AKT pathway is induced through loss of the negative regulator PTEN in $55 \%$ of cases (Lenz et al., 2008c; Pfeifer et al., 2013). The aberrations of Ga13 modulate the germinal center formation and cell movements but can also contribute to an enhanced PI3K-AKT pathway (Basso and DallaFavera, 2015; Muppidi et al., 2015; Muppidi et al., 2014).

While in ABC DLBCLs no deregulations of PTEN are found, the PI3K pathway activation is still increased due to receptor mutations of CD79a/b (Figure 2 B) (Kloo et al., 2011). These mutations in addition to receptor activation by self-antigens contribute to a constitutive activation of NF-KB, ERK and NFAT (Davis et al., 2010; Young et al., 2015). Beside the receptor activation, NF-KB can also been activated by modulation of CARD11 or MyD88. MyD88 induces NF-KB activation independent of upstream signals through IL1 receptor-associated kinase (IRAK) (Lenz et al., 2008a; Ngo et al., 2011). A further enhanced NF-kB signaling is caused by inactivation of the repressor A20 (Compagno et al., 2009). The constitutive activation of NF-KB leads to the expression of IL6 or IL10 and to a subsequent autocrine activation of STAT3 (Davis et al., 2001; Lam et al., 2008). Besides this NF-KB-dependent JAK-STAT activation, STAT3 mutations are also found in $A B C$ DLBCLs and indicate along with high IL10 serum levels a worse clinical outcome (Ding et al., 2008; Lech-Maranda et al., 2006). 
For the therapeutic aspect, the PI3K pathway is a promising drug target in GCB DLBCLs similar to BLs (Pfeifer et al., 2013). As the pathogenesis of GCB DLBCLs is not fully elucidated, it is not surprising that some GCB DLBCL cases with wild type PTEN show no PI3K-AKT-dependency. However, the response to PI3K pathway inhibitors can be traced back to the expression status of PTEN (Pfeifer et al., 2013). In ABC DLBCLs the BCRmediated NF-KB activation relies on BTK and PI3K activation. Inhibition of these kinases leads to a reduced proliferation in vitro except for cases with BCR-independent NF-KB activation (Wilson et al., 2015). MyD88 or CARD11 mutations causing a BCR-independent NF-KB activation can be counteracted through the inhibition of MALT1 or IRAK (Young and Staudt, 2013). As BTK inhibitors are well tolerable, the addition to standard therapies showed good results for B cell malignancies in the first clinical trials (Hendriks et al., 2014).
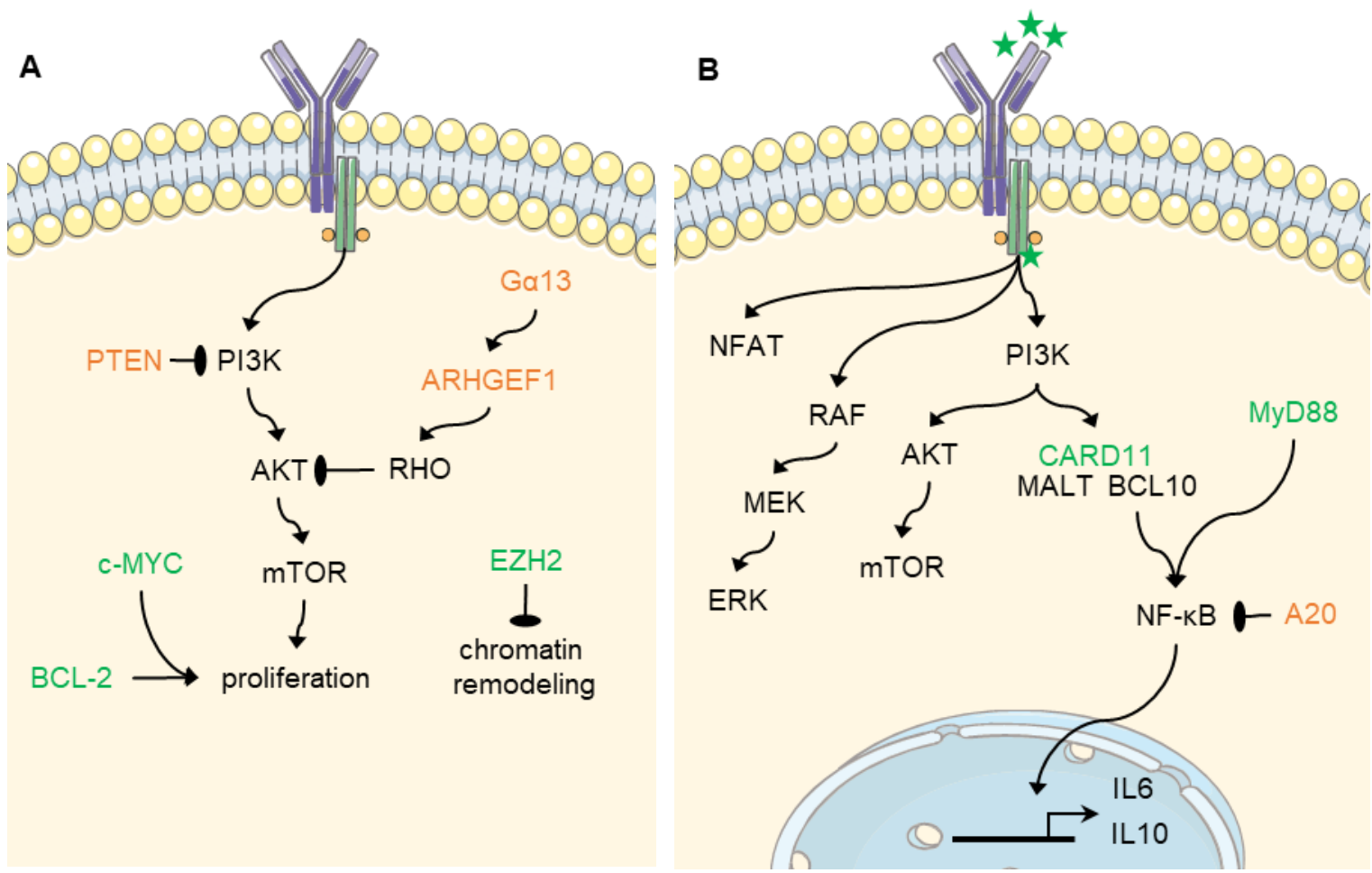

Figure 2: Schematic overview of some known deregulated signaling pathways in DLBCLs.

(A) The PI3K pathway is a major deregulated pathway of GCB DLBCLs. Missing negative interactions of Ga13 and PTEN contribute to the tonic BCR signaling. Aberrant regulation of C-MYC and BCL-2 enhance proliferation whereas EZH2 influences chromatin remodeling. (B) In ABC DLBCLs self-antigens and receptor mutations of $C D 79 \mathrm{a} / \mathrm{b}$ initiate a BCR signaling with activation of the PI3K-AKT, NF-KB, ERK and NFAT pathways. CARD11, MyD88 or A20 mutations contribute to an enhanced NF-KB activation which induces IL6 or IL10 expression. Black lines represent the interaction. Upregulated and downregulated proteins are displayed in green and red, respectively. Adapted from Basso and Rickert (Basso and Dalla-Favera, 2015; Rickert, 2013). 


\subsection{Important pathway interactions and crosstalks}

The inhibition of BCR-related kinases is promising for the treatment of several B cell malignancies. However, unexpected side effects and resistances occur consistently indicating disregarded feedback mechanisms (Blachly and Baiocchi, 2014). For cellular responses, the signal transmission from receptors to target gene expressions is not only a linear pathway. The signaling outcome is strongly regulated by different pathway kinetics and intensities. Thereby, the signal transduction from receptors to core kinase, the interaction between pathways and the modulation of feedbacks is essential to cause different reactions to extracellular stimuli (Bluthgen, 2015; Murphy and Blenis, 2006; Reth and Brummer, 2004). For this reason, signaling feedbacks and crosstalks can hamper targeted therapy through unknown effects. So far positive feedbacks of the BCR tyrosine kinases and the signalosome complex or negative feedbacks within one pathway have been described previously (Reth and Brummer, 2004). However, the downstream wiring of BCR-related pathways remains largely unexplored.

Beside its own negative feedback loop, the PI3K-AKT pathway interacts with the $\beta$-catenin pathway. AKT can phosphorylate and therefore inactivate glycogen synthase kinase-3 beta (GSK3 $\beta$ ) which is an inhibitory protein for $\beta$-catenin (Baracho et al., 2011). This leads to increased expression of cell cycle regulators like c-MYC and cyclin D3 and constitutes a further opportunity of the PI3K-AKT pathway to promote proliferation (Cato et al., 2011; Mazzoletti et al., 2011). Besides, a similar negative impact of ERK1/2 to GSK3 $\beta$ is described (Ding et al., 2005). The PI3K-AKT and MEK-ERK pathway share not only common downstream targets, they also influence each other. AKT can affect RAF phosphorylations leading to attenuation of the MEK-ERK pathway (Zimmermann and Moelling, 1999), whereas an ERK-dependent phosphorylation of MEK1 induces an interaction with PTEN at the membrane and causes a negative feedback on the PI3K-AKT pathway (Zmajkovicova et al., 2013).

Positive and negative feedbacks are not exclusively mediated through phosphorylation of target proteins. Both kinases and phosphatases must be precisely regulated to determine the duration and intensity of pathways and thereby the signaling output. Several phosphatases are known to intervene at any tier of signal transduction, however, a large number of phosphatases regulate especially MAPKs (Bluthgen, 2015; Junttila et al., 2008). Dual-specificity MAPK phosphatases (DUSP) specifically dephosphorylate threonine and tyrosine residues on different MAPK isoforms and can mediate the interplay between pathways (Jeffrey et al., 2007). For instance, in lung cancer cells DUSP1 is induced in an ERK-dependent manner by cisplatin. DUSP1 then decreases the pathway activity of p38 MAPK and JNK (Low and Zhang, 2016). Furthermore, the PI3K-AKT 
pathway can induce the degradation of the ERK-specific phosphatase DUSP6 and thus influences the ERK1/2 signal duration (Bermudez et al., 2008).

Despite first approaches analyzing the interactions of pathways, interplays and feedback loops are often disregarded in signaling schemes. Classical genetic or modern loss-offunction experiments only reveal the linear order of signaling elements. Therefore, studies investigating pathway inhibitions are needed for the detection of feedbacks (Reth and Brummer, 2004). Our group previously generated a network model based on gene expression effects after different pathway perturbations (Pirkl et al., 2016). However, this analysis could not fully elucidate the signaling network. To discover feedback loops and crosstalks the analysis of pathway activations seems to be indispensable. 


\section{Aims}

Many non-Hodgkin lymphomas are characterized by a strong dependency on the BCR signaling. The tonic BCR signaling is essential for the proliferation of Burkitt lymphoma and some GCB DLBCLs. In ABC DLBCLs mutations mimic a chronic active BCR, thereby causing NF-KB activation with subsequent JAK-STAT activation. While the proximal events and signaling cascades of the BCR are well studied, the downstream interplay of pathways is barely investigated although signaling feedbacks can promote or hinder the therapeutic success. Therefore, the establishment of reliable network models is useful to predict signaling alterations by external influences as well as therapeutic responses.

Our group has previously generated a Boolean Nested Effect model for BCR signaling from downstream gene expression changes of pathway perturbations (Pirkl et al., 2016). To verify and complement the signaling network structure and to generate more accurate network models, the interplay of BCR-related signaling pathways was analyzed in B cell lymphomas. Thus, the primary objective of this study focused on the following questions:

1. Which pathway interplays exist downstream of the tonic BCR signaling in BLs?

2. Which pathway interplays occur after activation of the BCR in BLs?

3. Is it possible to construct a general network model with pathway interplays?

4. Do pathway interplays affect cellular functions like proliferation?

5. Are pathway interplays similar for tonic, activated and chronic active BCR signaling?

To address these aims, an antibody-based screen of phosphoproteins was used to compare different pathway perturbations and selected interactions were investigated in more detail.

In addition, our group has demonstrated that a simultaneous activation of TLR9 and IL10R signaling synergistically enhances proliferation by influencing cell cycle genes and metabolism (Feist, 2016). We proposed that in addition to NF-KB and STAT3 phosphorylation the interplay of TLR9 and IL10R activation led to hitherto unconsidered protein phosphorylations. Therefore, the second part of my thesis concentrated on the following questions:

1. Which other mediators beside NF-KB and STAT3 promote the proliferative boost?

2. Which cellular processes are directly influenced by the pathway activations?

For this purpose, comprehensive mass spectrometry analysis of the phosphoproteom was performed in the model cell line P493-6 after stimulation of TLR9 and IL10R to imitate a combined activation of NF-KB and STAT3. 


\section{Material and Methods}

\subsection{Material, recipes and equipment}

\subsubsection{Biological material}

Cell lines used in the study are listed in table 1.

Table 1: Cell lines

\begin{tabular}{|c|c|c|c|}
\hline Cell line & Source & Distributor & Reference \\
\hline BL-2 & $\begin{array}{l}\text { Homo sapiens } \\
\text { Burkitt Lymphoma }\end{array}$ & $\begin{array}{l}\text { DSMZ, } \\
\text { Brunswick }\end{array}$ & (Bertrand et al., 1981) \\
\hline BL-41 & $\begin{array}{l}\text { Homo sapiens } \\
\text { Burkitt Lymphoma }\end{array}$ & $\begin{array}{l}\text { DSMZ, } \\
\text { Brunswick }\end{array}$ & (Lenoir et al., 1985) \\
\hline CA-46 & $\begin{array}{l}\text { Homo sapiens } \\
\text { Burkitt Lymphoma }\end{array}$ & $\begin{array}{l}\text { DSMZ, } \\
\text { Brunswick }\end{array}$ & (Magrath et al., 1980) \\
\hline $\mathrm{HBL}-1$ & $\begin{array}{l}\text { Homo sapiens } \\
\text { Diffuse Large B Cell Lymphoma (ABC) }\end{array}$ & $\begin{array}{l}\text { Krappmann, } \\
\text { Munich }\end{array}$ & (Nozawa et al., 1988) \\
\hline OCI-LY3 & $\begin{array}{l}\text { Homo sapiens } \\
\text { Diffuse Large B Cell Lymphoma (ABC) }\end{array}$ & $\begin{array}{l}\text { DSMZ, } \\
\text { Brunswick }\end{array}$ & (Tweeddale et al., 1987) \\
\hline P493-6 & $\begin{array}{l}\text { Homo sapiens } \\
\text { c-Myc transformed lymphoblastoid cell } \\
\text { line }\end{array}$ & $\begin{array}{l}\text { Bornkamm, } \\
\text { Munich }\end{array}$ & (Polack et al., 1996) \\
\hline
\end{tabular}

\subsubsection{Chemicals, solutions and consumable supplies}

The chemicals, solutions and supplies used are recorded in table 2, 3 and 4, respectively.

Table 2: Chemicals

\begin{tabular}{ll}
\hline Chemical & Manufacturer \\
\hline $\begin{array}{l}\text { 4-(2-hydroxyethyl)-1-piperazineethanesulfonic acid } \\
\text { (HEPES) }\end{array}$ & Sigma-Aldrich, St. Louis, US \\
4-lodophenylboronic acid (4-IPBA) & Sigma-Aldrich, St. Louis, US \\
Adenosine phosphosulfate (APS) & Serva, Heidelberg, DE \\
Albumin Fraction V (BSA) & Roth, Karlsruhe, DE \\
Ammonium sulfate & Merck KGaA, Darmstadt, DE \\
Bromophenol blue & Serva, Heidelberg, DE \\
Chameleon Duo Pre-stained Protein Ladder & LI-COR, Lincoln, US \\
cOmplete Mini & Roche, Basel, CH \\
Desoxyribonucleosid triphosphate (dNTP) & PrimeTech LTD, Minsk, BY \\
Diethylpyrocarbonate (DEPC) & Roth, Karlsruhe, DE \\
Dimethyl sulfoxide (DMSO) & Sigma-Aldrich, St. Louis, US \\
Dithiothreitol (DTT) & Sigma-Aldrich, St. Louis, US
\end{tabular}




\begin{tabular}{|c|c|}
\hline Chemical & Manufacturer \\
\hline DMSO cell culture grade & Sigma-Aldrich, St. Louis, US \\
\hline Ethanol & Roth, Karlsruhe, DE \\
\hline Ethylenediaminetetraacetic acid (EDTA) & Merck KGaA, Darmstadt, DE \\
\hline $\begin{array}{l}\text { Ethylene glycol bis(2-aminoethyl ether) tetraacetic acid } \\
\text { (EGTA) }\end{array}$ & Sigma-Aldrich, St. Louis, US \\
\hline Full range rainbow molecular weight marker & GE Healthcare, Chicago, US \\
\hline Glycerol & Merck KGaA, Darmstadt, DE \\
\hline Glycine & Roth, Karlsruhe, DE \\
\hline HEPES cell culture grade (Gibco) & Thermo Fisher, Waltham, US \\
\hline Hot FIREpol DNA polymerase (5 U/ $\mu \mathrm{l})$ & PrimeTech LTD, Minsk, BY \\
\hline Hydrochloric acid $37 \%$ & Merck KGaA, Darmstadt, DE \\
\hline Hydrogen peroxide $30 \%$ & Sigma-Aldrich, St. Louis, US \\
\hline Isopropanol & Roth, Karlsruhe, DE \\
\hline L-Arginine: $\mathrm{HCl}$ unlabeled & Euriso-Top, Saarbrücken, DE \\
\hline L-Arginine:HCl $\left({ }^{13} \mathrm{C} 6,99 \% ;{ }^{15} \mathrm{~N} 4,99 \%\right)$ & Euriso-Top, Saarbrücken, DE \\
\hline L-Lysine: $2 \mathrm{HCl}$ unlabeled & Euriso-Top, Saarbrücken, DE \\
\hline L-Lysine:2HCl ( $\left.{ }^{13} \mathrm{C} 6,99 \%\right)$ & Euriso-Top, Saarbrücken, DE \\
\hline L-Proline & Sigma-Aldrich, St. Louis, US \\
\hline Luminol & Sigma-Aldrich, St. Louis, US \\
\hline Magnesium chloride $\left(\mathrm{MgCl}_{2}\right)$ & Merck KGaA, Darmstadt, DE \\
\hline Meliseptol & B. Braun, Melsungen, DE \\
\hline Methanol & Roth, Karlsruhe, DE \\
\hline Nonylphenyl-polyethylene glycol (NP-40) & Sigma-Aldrich, St. Louis, US \\
\hline Phenylmethanesulfonyl fluoride (PMSF) & Sigma-Aldrich, St. Louis, US \\
\hline Phosphatase Inhibitor Cocktail 2 & Sigma-Aldrich, St. Louis, US \\
\hline Phosphatase Inhibitor Cocktail 3 & Sigma-Aldrich, St. Louis, US \\
\hline PhosSTOP & Roche, Basel, $\mathrm{CH}$ \\
\hline Potassium chloride (KCl) & Merck KGaA, Darmstadt, DE \\
\hline Sodium chloride $(\mathrm{NaCl})$ & Roth, Karlsruhe, DE \\
\hline Sodium dodecyl sulfate (SDS) & Serva, Heidelberg, DE \\
\hline Sodium fluoride & Sigma-Aldrich, St. Louis, US \\
\hline Sodium orthovanadate & Sigma-Aldrich, St. Louis, US \\
\hline Sodium pyruvate & Sigma-Aldrich, St. Louis, US \\
\hline SYBR green master mix fast & $\begin{array}{l}\text { Applied Biosystems, } \\
\text { Kalifornien, US }\end{array}$ \\
\hline Tetramethylethylenediamine (TEMED) & Sigma-Aldrich, St. Louis, US \\
\hline Trehalose dihydrate & Roth, Karlsruhe, DE \\
\hline $\begin{array}{l}\text { Tris(hydroxymethyl)-aminomethanhydrochlorid } \\
\text { (Tris-HCl) }\end{array}$ & Roth, Karlsruhe, DE \\
\hline TritonX-100 & Roth, Karlsruhe, DE \\
\hline Tween-20 & Serva, Heidelberg, DE \\
\hline Water HPLC grade & Th.Geyer, Renningen, DE \\
\hline Urea & Sigma-Aldrich, St. Louis, US \\
\hline
\end{tabular}


Table 3: Solutions

\begin{tabular}{ll}
\hline Solution & Manufacturer \\
\hline Acrylamide/Bis Solution $40 \%$ (w/v) & Serva, Heidelberg, DE \\
Dulbecco's Phosphate Buffered Saline (DPBS) & Lonza, Basel, CH \\
Fetal Bovine Serum (FBS) (Gibco) & Thermo Fisher, Waltham, US \\
Fetal Bovine Serum (FBS) Dialyzed (Gibco) & Thermo Fisher, Waltham, US \\
Odyssey Blocking Buffer & LI-COR, Lincoln, US \\
Penicillin and Streptomycin & Lonza, Basel, CH \\
Ponceau S Solution & Sigma-Aldrich, St. Louis, US \\
Re-Blot Plus Mild Solution (10x) & Merck Millipore, Burlington, US \\
RPMI-1640 with L-Glutamine & Lonza, Basel, CH \\
RPMI 1640 Media for SILAC & Thermo Fisher, Waltham, US \\
Roti-Load 1 (4x) & Roth, Karlsruhe, DE \\
Roti-Quant (5x) & Roth, Karlsruhe, DE \\
Trypan Blue Solution 0.4 \% & Sigma-Aldrich, St. Louis, US \\
& \\
\hline
\end{tabular}

Table 4: Consumables

\begin{tabular}{|c|c|}
\hline Consumable & Manufacturer \\
\hline Blotting paper BF3 & Th.Geyer, Renningen, DE \\
\hline C-Chip disposable hemocytometer NI & NanoEnTek, Waltham, US \\
\hline Cell culture flasks, suspension (T25, T75, T175) & Sarstedt, Nümbrecht, DE \\
\hline Combitips advanced $(0.5,5.0 \mathrm{ml})$ & Eppendorf, Hamburg, DE \\
\hline Cryo tubes $(2 \mathrm{ml})$ & Greiner Bio-One, Kremsmünster, AT \\
\hline Eppendorf tubes (5.0 ml) & Eppendorf, Hamburg, DE \\
\hline Falcon tubes $(15,50 \mathrm{ml})$ & Sarstedt, Nümbrecht, DE \\
\hline Filtropur S 0.2 & Sarstedt, Nümbrecht, DE \\
\hline Immobilon-FL transfer membrane PVDF $0.45 \mu \mathrm{m}$ & Merck KGaA, Darmstadt, DE \\
\hline Immobilon-P transfer membrane PVDF $0.45 \mu \mathrm{m}$ & Merck KGaA, Darmstadt, DE \\
\hline membranes ( $8 \mu \mathrm{m}$ pores) & Neuro Probe, Gaithersburg, US \\
\hline Micro tubes $(0.5,1.5,2.0 \mathrm{ml})$ & Sarstedt, Nümbrecht, DE \\
\hline Microplate PCR (384 well) & Greiner Bio-One, Kremsmünster, AT \\
\hline Microtest plate (96 well) & Sarstedt, Nümbrecht, DE \\
\hline Multiply- $\mu$ Strip pro 8-strip & Sarstedt, Nümbrecht, DE \\
\hline Optical adhesive covers & Applied Biosystems, Kalifornien, US \\
\hline Pasteur pipettes (150, 230 mm) & Th.Geyer, Renningen, DE \\
\hline Pipette tips $(20,200,1000 \mu \mathrm{l})$ & Sarstedt, Nümbrecht, DE \\
\hline Serological pipettes $(5,10,25 \mathrm{ml})$ & Sarstedt, Nümbrecht, DE \\
\hline Sterling nitrile powder-free exam gloves & Halyard Health, Georgia, US \\
\hline Syringe $(5.0,50 \mathrm{ml})$ & B. Braun, Melsungen, DE \\
\hline TipOne filter tips $(10,200,1000 \mu \mathrm{l})$ & Starlab, Hamburg, DE \\
\hline well plate, suspension, flat $(6,12,96$ well) & Sarstedt, Nümbrecht, DE \\
\hline well plate, suspension, round (96 well) & Sarstedt, Nümbrecht, DE \\
\hline
\end{tabular}




\subsubsection{Buffers and media}

The recipes of buffers used in the study are presented in table 5. All buffers are waterbased. For cell culture used media and supplements are listed in table 6.

Table 5: Recipes of buffers

\begin{tabular}{|c|c|}
\hline Buffer & Recipe \\
\hline chemiluminescence solution 1 & $\begin{array}{l}100 \mathrm{mM} \text { Tris } \mathrm{pH} 8.8 \\
2.5 \mathrm{mM} \text { Luminol } \\
4 \mathrm{mM} 4 \text {-IPBA }\end{array}$ \\
\hline chemiluminescence solution 2 & $\begin{array}{l}100 \mathrm{mM} \text { Tris } \mathrm{pH} 8.8 \\
9 \mathrm{mM} \text { Hydrogen peroxide }\end{array}$ \\
\hline NP-40 lysis buffer & $\begin{array}{l}50 \mathrm{mM} \text { Tris pH } 7.4 \\
150 \mathrm{mM} \mathrm{NaCl} \\
1 \mathrm{mM} \mathrm{EDTA} \\
0.50 \%(\mathrm{v} / \mathrm{v}) \mathrm{NP}-40 \\
0.1 \mathrm{mg} / \mathrm{ml} \mathrm{PMSF} \\
1 \times \mathrm{cOmplete} \text { Mini } \\
1 \times \text { PhosSTOP } \\
125 \mu \mathrm{M} \text { Sodium orthovanadate }\end{array}$ \\
\hline NP-40 lysis buffer modified & $\begin{array}{l}50 \mathrm{mM} \text { Tris } \mathrm{pH} 7.8 \\
150 \mathrm{mM} \mathrm{NaCl} \\
0.5 \mathrm{mM} \text { EDTA } \\
1 \%(\mathrm{v} / \mathrm{v}) \mathrm{NP}-40 \\
10 \%(\mathrm{v} / \mathrm{v}) \text { Glycerol } \\
2 \mathrm{mM} \text { Sodium orthovanadate } \\
1 \mathrm{mM} \text { Sodium fluoride } \\
1 \times \text { cOmplete Mini } \\
1 \times \text { Phosphatase Inhibitor Cocktail } 2 \\
1 \times \text { Phosphatase Inhibitor Cocktail } 3\end{array}$ \\
\hline nuclear extraction buffer $A$ & $\begin{array}{l}10 \text { mM HEPES pH } 7.9 \\
10 \text { mM KCl } \\
100 \mu \mathrm{M} \text { EDTA } \\
100 \mu \mathrm{M} \text { EGTA } \\
1 \text { mM DTT } \\
1 \text { x cOmplete Mini } \\
1 \times \text { PhosSTOP }\end{array}$ \\
\hline nuclear extraction buffer B & $\begin{array}{l}20 \mathrm{mM} \text { HEPES pH } 7.9 \\
400 \mathrm{mM} \mathrm{KCl} \\
1 \mathrm{mM} \text { EDTA } \\
1 \mathrm{mM} \text { EGTA } \\
1 \mathrm{mM} \text { DTT } \\
1 \times \text { cOmplete Mini } \\
1 \times \text { PhosSTOP }\end{array}$ \\
\hline
\end{tabular}




\begin{tabular}{|c|c|}
\hline Buffer & Recipe \\
\hline PCR buffer & $\begin{array}{l}750 \mathrm{mM} \text { Tris pH } 8.8 \\
200 \mathrm{mM} \text { Ammonium sulfate } \\
0.1 \%(\mathrm{v} / \mathrm{v}) \text { Tween-20 } \\
\text { in } 0.1 \%(\mathrm{w} / \mathrm{v}) \text { depc water }\end{array}$ \\
\hline resolving gel buffer & $\begin{array}{l}375 \mathrm{mM} \text { Tris pH } 8.8 \\
25 \%(\mathrm{v} / \mathrm{v}) \text { Acrylamide/Bis Solution (40\%) } \\
0.0004 \%(\mathrm{w} / \mathrm{v}) \text { APS } \\
0.00125 \%(\mathrm{v} / \mathrm{v}) \text { TEMED }\end{array}$ \\
\hline running buffer & $\begin{array}{l}25 \text { mM Tris } \\
192 \text { mM Glycine } \\
34.67 \text { mM SDS }\end{array}$ \\
\hline stacking gel buffer & $\begin{array}{l}125 \mathrm{mM} \text { Tris pH } 6.8 \\
12.5 \%(\mathrm{v} / \mathrm{v}) \text { Acrylamide/Bis Solution (40\%) } \\
0.0004 \%(\mathrm{w} / \mathrm{v}) \text { APS } \\
0.00125 \%(\mathrm{v} / \mathrm{v}) \text { TEMED }\end{array}$ \\
\hline SYBR green Mix & $\begin{array}{l}1 \times \text { PCR buffer } \\
3 \mathrm{mM} \mathrm{MgCl}_{2} \\
1: 80000 \text { SYBR green } \\
0.2 \mathrm{mM} \text { dNTP each } \\
20 \mathrm{U} / \mathrm{ml} \text { Hot FIREpol DNA polymerase } \\
0.25 \%(\mathrm{v} / \mathrm{v}) \text { TritonX-100 } \\
0.5 \mathrm{mM} \mathrm{Trehalose} \mathrm{dihydrate} \\
\text { in } 0.1 \%(\mathrm{w} / \mathrm{v}) \text { depc water }\end{array}$ \\
\hline Tris buffered saline (TBS) pH 7.6 & $\begin{array}{l}20 \mathrm{mM} \text { Tris } \\
137 \mathrm{mM} \mathrm{NaCl}\end{array}$ \\
\hline TBS-T & $\begin{array}{l}1 \times \text { TBS buffer } \\
0.1 \%(v / v) \text { Tween-20 }\end{array}$ \\
\hline $\begin{array}{l}\text { transfer buffer } \\
\mathrm{pH} 8.3\end{array}$ & $\begin{array}{l}25 \text { mM Tris } \\
192 \text { mM Glycine } \\
15 \%(v / v) \text { methanol }\end{array}$ \\
\hline urea buffer & $\begin{array}{l}25 \mathrm{mM} \text { Tris } \mathrm{pH} 8.0 \\
8 \mathrm{M} \text { urea } \\
1 \times \text { cOmplete Mini }\end{array}$ \\
\hline
\end{tabular}


Table 6: Media

\begin{tabular}{|c|c|}
\hline Medium & Recipe (Manufacturer) \\
\hline cell culture medium & $\begin{array}{l}\text { RPMI-1640 with L-Glutamine (Lonza) } \\
10 \%(\mathrm{v} / \mathrm{v}) \text { heat-inactivated FBS (Gibco) } \\
100 \mathrm{U} / \mathrm{ml} \text { Penicillin }+100 \mathrm{U} / \mathrm{ml} \text { Streptomycin }\end{array}$ \\
\hline cell labeling medium light & $\begin{array}{l}\text { RPMI } 1640 \text { Media for SILAC (Thermo Fisher) } \\
10 \%(\mathrm{v} / \mathrm{v}) \text { heat-inactivated FBS Dialyzed (Gibco) } \\
100 \mathrm{U} / \mathrm{ml} \text { Penicillin + } 100 \mathrm{U} / \mathrm{ml} \text { Streptomycin } \\
0.824 \mathrm{mM} \text { L-Arginine:HCl unlabeled } \\
0.275 \mathrm{mM} \text { L-Lysine:2HCl unlabeled } \\
200 \mathrm{mg} / \mathrm{L} \text { L-Proline }\end{array}$ \\
\hline cell labeling medium heavy & $\begin{array}{l}\text { RPMI } 1640 \text { Media for SILAC (Thermo Fisher) } \\
10 \% \text { (v/v) heat-inactivated FBS Dialyzed (Gibco) } \\
100 \mathrm{U} / \mathrm{ml} \text { Penicillin + } 100 \mathrm{U} / \mathrm{ml} \text { Streptomycin } \\
0.824 \mathrm{mM} \text { L-Arginine: } \mathrm{HCl}\left({ }^{13} \mathrm{C}_{6}, 99 \% ;{ }^{15} \mathrm{~N}_{4}, 99 \%\right) \\
0.275 \mathrm{mM} \text { L-Lysine:2HCl }\left({ }^{13} \mathrm{C}_{6}, 99 \%\right) \\
200 \mathrm{mg} / \mathrm{l} \text { L-Proline }\end{array}$ \\
\hline cell freezing medium & $\begin{array}{l}90 \%(\mathrm{v} / \mathrm{v}) \text { heat-inactivated FBS (Gibco) } \\
10 \%(\mathrm{v} / \mathrm{v}) \text { DMSO cell culture grade }\end{array}$ \\
\hline
\end{tabular}

\subsubsection{Cell culture supplements, inhibitors and siRNA}

In this study, cells were stimulated with soluble factors under the conditions listed in table 7. Inhibitors used to reduce different pathway activities are presented in table 8 with their respective working concentrations. Table 9 includes the small interfering RNA (siRNA) used for transient transfection analyses.

Table 7: Stimulants used in cell culture

\begin{tabular}{lll}
\hline Stimulant & $\begin{array}{l}\text { Working } \\
\text { concentration }\end{array}$ & Manufacturer \\
\hline $\begin{array}{l}\text { anti-human IgM F(ab')2 } \\
\text { (a-IgM) Fragment }\end{array}$ & $13 \mu \mathrm{g} / \mathrm{ml}$ & $\begin{array}{l}\text { Jackson ImmunoResearch, } \\
\text { Cambridgeshire, GB } \\
\text { CpG ODN2006 }\end{array}$ \\
$\begin{array}{l}\text { Doxycycline } \\
\text { recombinant human IL10 }\end{array}$ & $\begin{array}{l}0.5 \mu \mathrm{ng} \\
10 \mathrm{ng} / \mathrm{ml}\end{array}$ & $\begin{array}{l}\text { Clontech, Saint-Germain-en-Laye, FR } \\
\text { Peprotech, Rocky Hill, US }\end{array}$ \\
\hline
\end{tabular}


Table 8: Inhibitors used in cell culture

\begin{tabular}{|c|c|c|c|}
\hline Inhibitor & Target & $\begin{array}{l}\text { Working } \\
\text { concentration }\end{array}$ & Manufacturer \\
\hline (5Z)-7-Oxozeaenol & TAK1 & $0.5 \mu \mathrm{M}$ & Tocris Bioscience, Bristol, GB \\
\hline $\mathrm{ACHP}$ & $\mathrm{IKK} \beta$ & $7 \mu \mathrm{M}$ & Merck KGaA, Darmstadt, DE \\
\hline AZD6244 & MEK 1,2 & $1 \mu \mathrm{M}$ & Selleckchem, Munich, DE \\
\hline AZ-TAK1 & TAK1 & $0.5 \mu \mathrm{M}$ & Abcam, Cambridge, GB \\
\hline BKM120 & p100 $\alpha, \delta, \beta, \gamma$ & $1 \mu \mathrm{M}$ & Selleckchem, Munich, DE \\
\hline CAL-101 & p100 $\delta, \gamma$ & $1 \mu \mathrm{M}$ & Selleckchem, Munich, DE \\
\hline Ibrutinib & BTK & $10 \mu \mathrm{M}$ & Selleckchem, Munich, DE \\
\hline JNK Inhibitor VIII & JNK 3,1,2 & $5 \mu \mathrm{M}$ & Merck KGaA, Darmstadt, DE \\
\hline LY294002 & p100 $\alpha, \delta, \beta$ & $10 \mu \mathrm{M}$ & Merck KGaA, Darmstadt, DE \\
\hline MK-2206 & AKT 1,2,3 & $1 \mu \mathrm{M}$ & Selleckchem, Munich, DE \\
\hline MLN120B & $\mathrm{IKK} \beta$ & $10 \mu \mathrm{M}$ & MedChemExpress, Sollentuna, SE \\
\hline Rapamycin & mTOR & $1 \mu \mathrm{M}$ & Selleckchem, Munich, DE \\
\hline SB203580 & p38 $\alpha, \beta$ & $2 \mu \mathrm{M}$ & Sigma-Aldrich, St. Louis, US \\
\hline SP600125 & JNK 1,2,3 & $5 \mu \mathrm{M}$ & Merck KGaA, Darmstadt, DE \\
\hline U0126 & MEK 2,1 & $10 \mu \mathrm{M}$ & Sigma-Aldrich, St. Louis, US \\
\hline
\end{tabular}

Table 9: siRNA

\begin{tabular}{lll}
\hline siRNA & Manufacturer & Order no. \\
\hline MAPK14 (p38 MAPK) & Dharmacon, Colorado, US & L-003512-00-0005 \\
negative control (scrb) & Life Technologies, Carlsbad, US & 4390844 \\
\hline
\end{tabular}

\subsubsection{Antibodies}

The detection of phosphorylated or total proteins was conducted in the study with antibodies listed in table 10.

Table 10: Antibodies

\begin{tabular}{|c|c|c|c|c|}
\hline Antibody against & $\begin{array}{l}\text { Host } \\
\text { species }\end{array}$ & Dilution & Order no. & Source \\
\hline AKT & rabbit & $1: 1000$ & 9272 & $\begin{array}{l}\text { Cell Signaling Technology, } \\
\text { Leiden, NL }\end{array}$ \\
\hline p-AKT (Ser473) & rabbit & $1: 1000$ & 9271 & $\begin{array}{l}\text { Cell Signaling Technology, } \\
\text { Leiden, NL }\end{array}$ \\
\hline $\begin{array}{l}\text { Histone Deacetylase } 1 \\
\text { (HDAC1) }\end{array}$ & rabbit & $1: 1000$ & 2062 & $\begin{array}{l}\text { Cell Signaling Technology, } \\
\text { Leiden, NL }\end{array}$ \\
\hline MEK1/2 & rabbit & 1:1000 & 9122 & $\begin{array}{l}\text { Cell Signaling Technology, } \\
\text { Leiden, NL }\end{array}$ \\
\hline
\end{tabular}




\begin{tabular}{|c|c|c|c|c|}
\hline Antibody against & $\begin{array}{l}\text { Host } \\
\text { species }\end{array}$ & Dilution & Order no. & Source \\
\hline $\begin{array}{l}\text { p-MEK1/2 (Ser217/221) } \\
\text { (41G9) }\end{array}$ & rabbit & $1: 1000$ & 9154 & $\begin{array}{l}\text { Cell Signaling Technology, } \\
\text { Leiden, NL }\end{array}$ \\
\hline Mouse IgG-HRP & goat & $1: 2000$ & sc-2005 & $\begin{array}{l}\text { Santa Cruz Biotechnology, } \\
\text { Dallas, US }\end{array}$ \\
\hline Mouse IRDye 680RD & goat & $1: 15000$ & $\begin{array}{l}925- \\
68070\end{array}$ & LI-COR, Lincoln, US \\
\hline p38 MAPK & rabbit & $1: 1000$ & 9212 & $\begin{array}{l}\text { Cell Signaling Technology, } \\
\text { Leiden, NL }\end{array}$ \\
\hline $\begin{array}{l}\text { p-p38 MAPK } \\
\text { (Thr180,Tyr182) (D3F9) }\end{array}$ & rabbit & $1: 1000$ & 4511 & $\begin{array}{l}\text { Cell Signaling Technology, } \\
\text { Leiden, NL }\end{array}$ \\
\hline $\begin{array}{l}\text { p44/p42 (ERK1/2) } \\
(\mathrm{L} 34 \mathrm{~F} 12)\end{array}$ & mouse & $1: 1000$ & 4696 & $\begin{array}{l}\text { Cell Signaling Technology, } \\
\text { Leiden, NL }\end{array}$ \\
\hline $\begin{array}{l}\text { p-p44/p42 (pERK1/2) } \\
\text { (Thr202/Tyr204) (197G2) }\end{array}$ & rabbit & 1:1000 & 4377 & $\begin{array}{l}\text { Cell Signaling Technology, } \\
\text { Leiden, NL }\end{array}$ \\
\hline p70 S6 Kinase (49D7) & rabbit & 1:1000 & 2708 & $\begin{array}{l}\text { Cell Signaling Technology, } \\
\text { Leiden, NL }\end{array}$ \\
\hline $\begin{array}{l}\text { p-p70 S6 Kinase (Thr389) } \\
\text { (108D2) }\end{array}$ & rabbit & $1: 1000$ & 9234 & $\begin{array}{l}\text { Cell Signaling Technology, } \\
\text { Leiden, NL }\end{array}$ \\
\hline Rabbit IgG-HRP & goat & $1: 2000$ & sc-2004 & $\begin{array}{l}\text { Santa Cruz Biotechnology, } \\
\text { Dallas, US }\end{array}$ \\
\hline Rabbit IRDye 800CW & goat & $1: 15000$ & $\begin{array}{l}925- \\
32211\end{array}$ & LI-COR, Lincoln, US \\
\hline p-Raf1 (Ser289/296/301) & rabbit & 1:1000 & 9431 & $\begin{array}{l}\text { Cell Signaling Technology, } \\
\text { Leiden, NL }\end{array}$ \\
\hline p-Raf1 (Ser338) (56A6) & rabbit & 1:1000 & 9427 & $\begin{array}{l}\text { Cell Signaling Technology, } \\
\text { Leiden, NL }\end{array}$ \\
\hline $\begin{array}{l}\text { S6 ribosomal protein } \\
\text { (54D2) }\end{array}$ & mouse & $1: 1000$ & 2317 & $\begin{array}{l}\text { Cell Signaling Technology, } \\
\text { Leiden, NL }\end{array}$ \\
\hline $\begin{array}{l}\text { p-S6 ribosomal protein } \\
\text { (Ser240/244) }\end{array}$ & rabbit & $1: 1000$ & 2215 & $\begin{array}{l}\text { Cell Signaling Technology, } \\
\text { Leiden, NL }\end{array}$ \\
\hline TAK1 (D94D7) & rabbit & 1:1000 & 5206 & $\begin{array}{l}\text { Cell Signaling Technology, } \\
\text { Leiden, NL }\end{array}$ \\
\hline Tubulin & mouse & $1: 1000$ & $05-829$ & $\begin{array}{l}\text { Merck Millipore, Burlington, } \\
\text { US }\end{array}$ \\
\hline
\end{tabular}




\subsubsection{Oligonucleotides}

For quantitative real-time PCR (qRT-PCR), oligonucleotides listed in table 11 were applied. All oligonucleotides were purchased from IBA Lifesciences (Goettingen, DE).

Table 11: Oligonucleotides

\begin{tabular}{lll}
\hline Gene & Forward primer (5' - 3') & Reverse primer (5' - 3') \\
\hline EGR2 & GCA CCA GCT GTC TGA CAA CAT CT & CAT GTC AAT GTT GAT CAT GCC ATC \\
EGR3 & CGG TGA CCA TGA GCA GTT TG & GTA GGT CAC GGT CTT GTT GC \\
FOS & GCT TCA ACG CAG ACT ACG AG & AGT GAC CGT GGG AAT GAA GT \\
GAPDH & CAG CCT CAA GAT CAT CAG CA & CAT GAG TCC TTC CAC GAT ACC \\
MAP2K6 & GTG AAG GCA GAT GAC CTG GAG & GGA TCC GCT TCA CTG CCA T \\
PDP1 & CCA GAC GAA TTG GAA TCC CAG & AGT GCC ATA GAT CCT GCT CAG TTC \\
PLD6 & CAA ATC GGT CTG CTG CGC & AGT GAT GAG CAC CCT CTT GTC C \\
PTGS1 & AGC AGA GTT GGA GGA ATT GTA & CAG GGT AGA ACT CCA ACG CAT C \\
& TGG & \\
TNFa & TCT CTA ATC AGC CCT CTG G & CTA CAA CAT GGG CTA CAG G \\
ZFP36L1 & TCT GCC ACC ATC TTC GAC TT & GTC TTG TAG CGG CTG GAG TT \\
&
\end{tabular}

\subsubsection{Ready to use reaction systems}

Assays in the study were conducted with ready to use reaction systems which are shown in table 12.

Table 12: Reaction systems

\begin{tabular}{lll}
\hline Description & Manufacturer & Order no. \\
\hline Amaxa Cell Line Nucleofector Kit V & Lonza, Basel, CH & VCA-1003 \\
BCA Protein Assay & Thermo Fisher, Waltham, US & 23225 \\
Bio-Plex Pro Cell Signaling Reagent & BIO-RAD, Hercules, US & $171-304006 \mathrm{M}$ \\
Calcein AM Cell Viability & R\&D Systems, Minneapolis, US & \\
NucleoSpin RNA & Macherey-Nagel, Düren, DE & 740.955 .250 \\
SuperScript II Reverse Transcriptase & Invitrogen, Carlsbad, US & $18064-014$ \\
& & \\
\hline
\end{tabular}




\subsubsection{Equipment}

Analyses in the study were done with the equipment listed in table 13.

Table 13: Equipment

\begin{tabular}{|c|c|}
\hline Instrument & Manufacturer \\
\hline 7900HT Fast Real-Time PCR System & Thermo Fisher, Waltham, US \\
\hline Balance Kern EW420-3NM & Kern\&Sohn, Balingen, DE \\
\hline Bio-Plex Protein Array system & BIO-RAD, Hercules, US \\
\hline Centrifuge Heraeus Fresco 21 & Thermo Fisher, Waltham, US \\
\hline Centrifuge Heraeus Multifuge $3 \mathrm{~L}-\mathrm{R}$ & Thermo Fisher, Waltham, US \\
\hline Centrifuge Heraeus Multifuge X3R & Thermo Fisher, Waltham, US \\
\hline Counting chamber Neubauer Improved & LO LaborOptik, Friedrichsdorf, DE \\
\hline Freezer $\left(-80^{\circ} \mathrm{C}\right)$ & Panasonic Corporation, Osaka, JP \\
\hline Heraeus BB6220 & Thermo Fisher, Waltham, US \\
\hline IKAMAG RCT & IKA Works, Staufen, DE \\
\hline Image Reader LAS-4000 mini & Fujifilm, Tokio, JP \\
\hline Infinite F50 & Tecan Group, Männedorf, CH \\
\hline Laminar flow Telstar Bio-II-A & Prettl, Pfullingen, DE \\
\hline Micro centrifuge $1-15 \mathrm{~K}$ & Sigma-Aldrich, St. Louis, US \\
\hline Micro centrifuge 220 VAC & Roth, Karlsruhe, DE \\
\hline Micro centrifuge 5424 & Eppendorf, Hamburg, DE \\
\hline Micro Chemotaxis Chamber (48-well) & Neuro Probe, Gaithersburg, US \\
\hline Microscope Telaval 31 & Zeiss, Oberkochen, DE \\
\hline Mini Trans-Blot Cell & BIO-RAD, Hercules, US \\
\hline Multipette plus & Eppendorf, Hamburg, DE \\
\hline Nalgene Cryo $1^{\circ} \mathrm{C}$ Freezing Container & Thermo Fisher, Waltham, US \\
\hline Nucleofector $2 b$ & Lonza, Basel, CH \\
\hline Odyssey CLx & LI-COR, Lincoln, US \\
\hline pH-Meter 761 Calimatic & Knick, Berlin, DE \\
\hline Pipette 8-channel (1-10 $\mu \mathrm{l})$ & Eppendorf, Hamburg, DE \\
\hline Pipette 8-channel (10-100 $\mu$ l) ErgoOne & Starlab, Hamburg, DE \\
\hline Power Pac 300 & BIO-RAD, Hercules, US \\
\hline Power Supply EV202 & Consort bvba, Turnhout, BE \\
\hline Roller Mixer SRT6 & Stuart, Staffordshire, GB \\
\hline Shaker 3005 & GFL, Burgwedel, DE \\
\hline Spectrophotometer ND-1000 & Thermo Fisher, Waltham, US \\
\hline Synergy HTX multi-mode reader & BioTek, Winooski, US \\
\hline Biometra Thermocycler T3000 & Analytik Jena, Jena, DE \\
\hline UVC/T-AR, DNA/RNA UV-cleaner box & BioSan, Riga, LV \\
\hline Vortex Genius 3 & IKA Works, Staufen, DE \\
\hline Water bath & Köttermann, Uetze, DE \\
\hline
\end{tabular}




\subsubsection{Software}

Software presented in table 14 was used for analysis and visualization of the obtained data.

Table 14: Software

\begin{tabular}{ll}
\hline Software & Developer \\
\hline ABI 7900HT SDS 2.4 & Applied Biosystems, Kalifornien, US \\
ABI RQ Manager 1.2.1 & Applied Biosystems, Kalifornien, US \\
Adobe Illustrator CS6 Version 16.0.0 & Adobe Systems, Kalifornien, US \\
Adobe Photoshop CS2 Version 9.0 & Adobe Systems, Kalifornien, US \\
Bio-Plex Manager Software & BIO-RAD, Hercules, US \\
EndNote X5 & Clarivate Analytics, Pennsylvania, US \\
Gen5 2.0 & BioTek, Winooski, US \\
GraphPad Prism Version 7.03 & GraphPad Software, La Jolla, US \\
Image Studio Lite Version 5.2.5 & LI-COR, Lincoln, US \\
Microsoft Office Professional Plus 2016 & Microsoft, Washington, US \\
(Excel, Word, PowerPoint) & \\
Magellan for F50 Version 7.0 & Tecan Group, Männedorf, CH \\
LAS-4000 mini Version2.0 & Fujifilm, Tokio, JP \\
NanoDrop 1000 3.8.1 & Thermo Fisher, Waltham, US \\
& \\
\hline
\end{tabular}

\subsection{Cell Biology}

\subsubsection{Cell Culture}

All cell lines used in this study were grown in cell culture medium at $37^{\circ} \mathrm{C}$ and $5 \% \mathrm{CO}_{2}$. The cells were kept in culture for up to four weeks. BLs were cultivated at a density of $1.5 \times 10^{5}$ to $1.5 \times 10^{6} \mathrm{cells} / \mathrm{ml}$ by splitting three times a week. ABC DLBCLs and P493-6 cells were maintained at a higher density of $3 \times 10^{5}$ to $1.5 \times 10^{6} \mathrm{cells} / \mathrm{ml}$. To determine the cell numbers a hemocytometer was used for counting and dead cells were excluded by trypan blue. For experiments, cells were freshly adjusted the day before. Furthermore, the MYC overexpressing P493-6 cells were supplemented with $1 \mathrm{ng} / \mathrm{ml}$ doxycycline 16 hours prior to the experiment to obtain a c-Myc depleted condition.

For long-term storage, the cell lines were frozen. For this propose, cells were centrifuged (100 $\mathrm{xg}, \mathrm{RT})$ for 5 minutes and resuspended in cell freezing medium. Suspensions of $1 \mathrm{ml}$ containing $3 \times 10^{6}$ cells of BLs or $5 \times 10^{6}$ cells of DLBCLs/P493-6 were cooled down to $-80^{\circ} \mathrm{C}$ using a cryo freezing container. The cryo freezing container filled with isopropanol provides a constant cooling of $-1^{\circ} \mathrm{C}$ per minute. After 24 hours the cryo tubes were placed at $-150^{\circ} \mathrm{C}$ for long-term storage. The thawing was performed rapidly in a 
$37^{\circ} \mathrm{C}$ water bath and the $1 \mathrm{ml}$ cell suspension was transferred in $9 \mathrm{ml}$ cell culture medium. After centrifugation (100 $\mathrm{g}$, RT) for 5 minutes the cells were resuspended in fresh cell culture medium and adjusted to their optimal density.

\subsubsection{Stable isotope labeling by amino acids in cell culture}

For phosphoproteomics, stable isotope labeling by amino acids in cell culture (SILAC) was used to analyze two conditions simultaneously by mass spectrometry. The incorporation of ${ }^{13} \mathrm{C}$ - and ${ }^{15} \mathrm{~N}$-labeled amino acids during protein turnover leads to a distinct mass difference between the samples making them quantitative and accurate comparable (Mann, 2006). P493-6 cells were thawed and cultured in cell culture medium for three days. Afterwards the cell medium was replaced by either cell labeling medium light or cell labeling medium heavy for seven days to fully incorporate the label into proteins. To avoid an arginine to proline conversion, the labeling medium was supplemented with $200 \mathrm{mM}$ L-proline. After six days and 16 hours prior to the experiment, the cells were centrifuged $(100 \times \mathrm{g}, \mathrm{RT})$ for 5 minutes and freshly adjusted to $8 \times 10^{5} \mathrm{cell} / \mathrm{ml}$. Besides, the lightlabeled cells were stimulated with $1 \mathrm{ng} / \mathrm{ml}$ doxycycline to obtain a c-Myc depleted condition.

\subsubsection{Inhibitor treatment and stimulation of the BCR signaling}

The inhibition of distinct pathway activations was done with inhibitors which are displayed with their working concentrations in Table 8. As all inhibitors are solved in DMSO, the control cells were always treated with the equal volume of DMSO. The different cell lines were seeded in fresh cell culture medium and adjusted to a density of $1 \times 10^{6} \mathrm{cells} / \mathrm{ml}$. For studying protein phosphorylations, the cells were incubated with inhibitors for 3 hours. HBL-1 and OCI-LY3 were then harvest while BLs were incubated for additional 30 minutes with or without BCR activation. The BCR activation was done by stimulation with $13 \mu \mathrm{g} / \mathrm{ml}$ anti-human $\lg \mathrm{F}\left(\mathrm{ab}^{\prime}\right) 2$ for 5 or 30 minutes. For RNA analysis, inhibitors were supplemented 3 hours before the BCR was activated for 1 - 3 hours.

\subsubsection{IL10 and CpG stimulation}

For phosphoproteomics, isotope labeled P493-6 cells were counted and 20 million heavylabeled cells stimulated with $40 \mathrm{ng} / \mathrm{ml} \mathrm{IL10}$ and $0.5 \mu \mathrm{M} \mathrm{CpG}$ as control reference. The light-labeled cells were divided in two samples of 10 million cells respectively. While one was treated with $40 \mathrm{ng} / \mathrm{ml} \mathrm{IL} 10$ and $0.5 \mu \mathrm{M} \mathrm{CpG}$, the other was equally stimulated with DPBS $+0.1 \%$ BSA as control. After 30 minutes of pathway activation cells were harvested for mass spectrometry analysis. 
For studying the impact of STAT3 and NF-KB activation on the migration capacity, c-Myc depleted P493-6 cells were counted and centrifuged (100 x g, RT) for 5 minutes. After adjustment to a density of $1 \times 10^{6} \mathrm{cells} / \mathrm{ml}$ in FBS-free cell culture medium, P493-6 cells were supplemented with $1 \mathrm{ng} / \mathrm{ml}$ doxycycline to maintain the c-Myc depleted condition. Furthermore, the cells were stimulated with $40 \mathrm{ng} / \mathrm{ml} \mathrm{IL10}$ and $0.5 \mu \mathrm{M} \mathrm{CpG}$ and the control cells were treated with equal amounts of DPBS $+0.1 \%$ BSA. The migration assay was performed immediately.

\subsubsection{RNA-interference-mediated gene knockdown}

To down regulate specific proteins, small interfering RNAs (siRNA) were transferred by an electroporation-based method called Nucleofector ${ }^{\mathrm{TM}}$ Technology (Lonza). The combination of unique buffers with cell-specific electroporation programs ensures high transfection efficiencies especially for suspension cells. For the BL-2 cell line the Amaxa Cell Line Nucleofector Kit V (Lonza) and the Nucleofector 2b (Lonza) with program R-013 was used. According to the manufacturer's transfection protocol, 2 million cells were centrifuged (90 x g, RT) for 10 minutes and resuspended in $100 \mu \mathrm{l}$ Nucleofector Solution $\mathrm{V}$ with $2 \mu \mathrm{g}$ siRNA. The cells were immediately transfected and received in pre-warmed cell culture medium supplemented with $10 \mathrm{mM}$ HEPES and $1 \mathrm{mM}$ sodium pyruvate. After 24 hours cells were stimulated with $13 \mu \mathrm{g} / \mathrm{ml}$ anti-human $\lg M F(a b ') 2$ for 30 minutes and harvested for protein phosphorylation analysis.

\subsubsection{Cell viability assay}

The cell viability after inhibitor treatment was determined using the Calcein AM Cell Viability Assay (R\&D Systems). Calcein AM is a non-fluorescent, cell-permeable compound that is converted to a green-fluorescent dye by esterases of living cells. The inhibition of distinct pathway activations was done with inhibitors which are displayed with their working concentrations in Table 8. As all inhibitors are solved in DMSO, the control cells were always treated with the equal volume of DMSO. Inhibitor treated and control cells were seeded as triplicates in a 96-well plate. BLs were adjusted to $7.5 \times 10^{3}$ cells/well and ABC DLBCLs to $1.5 \times 10^{4}$ cells/well in a final volume of $50 \mu$ l. After certain time points, $50 \mu \mathrm{l} 1 \times$ Calcein buffer were added to each well and the plate was centrifuged ( $200 \times \mathrm{g}, \mathrm{RT})$ for 3 minutes. The supernatant was removed and the cells incubated in $100 \mu \mathrm{l} 1 \times$ Calcein buffer with $0.1 \mu \mathrm{M}$ Calcein $\mathrm{AM}$ for 20 minutes at $37^{\circ} \mathrm{C}$. Three wells without cells were additionally filled as blank value. Punctually, the absorption at $485 \mathrm{~nm}$ was measured and the viability calculated. For this purpose, the blank value was subtracted from sample values and all treatments normalized to the control. 


\subsubsection{Migration assay}

The migration potential of cells towards a stimulus was studied with the Boyden Chamber assay (Chen, 2005). The boyden chamber consists of two chambers separated by a porous membrane. Therefore, the movement of cells through pores of a defined size can be regarded. For the P493-6 cell line, a 48-well micro chemotaxis chamber and membranes with $8 \mu \mathrm{m}$ pore size were used. The lower wells were filled with either cell culture medium containing $10 \%$ FCS as directed migration or medium without FCS as undirected condition. After placing the membrane and sealing the chamber, the upper wells were filled with cell suspension. The cell suspension contained $1 \times 10^{6} \mathrm{cells} / \mathrm{ml}$ in FCS-free cell culture medium. The chamber was then incubated at $37^{\circ} \mathrm{C}$ and the cells were allowed to migrate for 6 hours. Afterwards, the chamber was disassembled and the cell concentration of the lower wells was determined. For each condition six technical replicates were evaluated by cell counting using disposable hemocytometer chips. The cell amount of each condition was normalized to the directed control condition.

\subsection{Protein biochemistry}

\subsubsection{Preparation of cell lysates and cell fractionation for Western Blot analysis}

After stimulation, inhibitor treatment or siRNA transfection cells were harvested for pathway analysis. To protect protein phosphorylation cells were firstly cooled down on ice by addition of the two-fold volume of ice-cold DPBS supplemented with $0.5 \times$ PhosSTOP (Roche) and $100 \mu \mathrm{M}$ sodium orthovanadate. Furthermore, the cells were centrifuged $\left(500 \times \mathrm{g}, 4^{\circ} \mathrm{C}\right)$ for 5 minutes, washed once and the dry pellet stored at $-80^{\circ} \mathrm{C}$. For lysis, the cell pellet was resuspended in $50 \mu \mathrm{l}$ cold NP-40 lysis buffer and shaken for 30 minutes on ice. The debris was removed by centrifugation $\left(14.000 \times \mathrm{g}, 4^{\circ} \mathrm{C}\right)$ for 15 minutes and the supernatant transferred into new tubes. The protein concentration was determined with Roti-Quant (Roth) according to the manufacturer's instructions for use. Protein concentration of all samples were adjusted to each other with lysis buffer.

To separate cytosolic and nuclear fractions cells were burst by swelling and intact nuclei isolated as described by Schreiber et al. (Schreiber et al., 1989). After stimulation or inhibitor treatment, cells were cooled down on ice by addition of the one-fold volume of ice-cold DPBS supplemented with $0.5 \times$ PhosSTOP (Roche) and $100 \mu \mathrm{M}$ sodium orthovanadate. The cells were centrifuged $\left(500 \times \mathrm{g}, 4^{\circ} \mathrm{C}\right)$ for 5 minutes and washed once. In order to burst the cell membrane $200 \mu$ l nuclear extraction buffer A were added and samples were shaken 15 minutes on ice. Immediately, samples were supplemented with $12.5 \mu \mathrm{l} 10 \% \mathrm{NP}-40$, mixed by vortexing and the nuclei were collected by centrifugation 
$\left(14000 \times \mathrm{g}, 4^{\circ} \mathrm{C}\right)$ for 1 minute. The supernatant containing the cytosolic fraction was transferred to a new tube. The pellets were washed with $50 \mu \mathrm{l}$ nuclear extraction buffer $A$ and centrifuged $\left(14000 \times \mathrm{g}, 4^{\circ} \mathrm{C}\right)$ for 1 minute. After discarding the supernatant pellets were lysed in $30 \mu \mathrm{l}$ nuclear extraction buffer $B$ for 25 minutes while shaking on ice. Finally, the debris was removed by centrifugation $\left(14000 \times \mathrm{g}, 4^{\circ} \mathrm{C}\right)$ for 5 minutes and the supernatant with the nuclear fraction transferred to a new tube. The protein concentration of both fractions was determined with Roti-Quant (Roth) according to the manufacturer's instructions for use. Protein concentrations of all samples were adjusted to each other with the respective buffer.

\subsubsection{SDS Page, Western Blot and Immunodetection}

The separation of proteins was done by a discontinuous sodium dodecyl sulfate (SDS) polyacrylamide gel electrophoresis (Page) (Laemmli, 1970). For this purpose, modified buffers were used to generate a $10 \%$ resolving gel with a $5 \%$ stacking gel at least 24 hours in advance. The samples were supplemented with $1 \times$ Roti-Load (Roth) and heated to $95^{\circ} \mathrm{C}$ for 5 minutes just before use. The gel was loaded with $20 \mu \mathrm{g}$ protein and a molecular weight marker to determine protein size. For separation, the chamber was filled with $1 \mathrm{x}$ running buffer and a current of $30 \mathrm{~mA}$ for 20 minutes was firstly used. Furthermore, the current was set to $20 \mathrm{~mA}$ for 1 hour. The voltage was limited to $150 \mathrm{~V}$ to avoid overheating and the run was stopped after the dye front had left the gel.

In order to transfer the separated proteins to a hydrophobic membrane, the tank transfer system was used (Towbin et al., 1979). In deviation to this, a PVDF membrane was taken which needs to be activated by $100 \%$ methanol for 20 seconds, to be rehydrated in $\mathrm{dd}_{2} \mathrm{O}$ for 2 minutes and to be equilibrated in transfer buffer for 5 minutes. Meanwhile, the SDS page gels were incubated in transfer buffer and the stacking gel was cut off. After blotting at $4^{\circ} \mathrm{C}$ and $100 \mathrm{~V}$ for 1 hour the membranes were removed and air-dried for 30 minutes. For re-wetting, membranes were shortly incubated in $100 \%$ methanol and washed twice with TBS for 5 minutes. Blocking of unspecific binding sites was done with Odyssey Blocking Buffer or $5 \%$ BSA in TBS for 1 hour (RT).

To detect the protein of interest the membranes were incubated overnight $\left(4^{\circ} \mathrm{C}\right)$ in $5 \%$ BSA-TBS-T with the primary antibody (Table 10). After washing 3 times for 5 minutes with TBS-T the suitable secondary antibody in $5 \%$ BSA-TBS-T was added for 1 hour (RT). Finally, the membranes were washed further 3 times and the secondary antibodies visualized either by chemiluminescence or fluorescence detection. For chemiluminescence, the self-prepared chemiluminescence solutions 1 and 2 were mixed in equal parts according to Haan \& Behrmann (Haan and Behrmann, 2007) and the Image 
Reader LAS-4000 mini (Fujifilm) was used. The detection of fluorescence was performed light-protected with the Odyssey CLx (LI-COR). After staining of protein phosphorylations, the membranes were reblotted 15 minutes in $1 \times$ Re-Blot Plus Mild Solution and washed three times with TBS. Before analyzing the total protein, the membrane was blocked in Odyssey Blocking Buffer or $5 \%$ BSA in TBS for 1 hour (RT). Then the next antibody detection could be performed overnight as described above.

\subsubsection{Bio-Plex ${ }^{\circledR}$ Multiplex Immunoassay}

To measure 15 different protein phosphorylations in one sample, the magnetic beadbased multiplex assay (BIO-RAD) was performed in close collaboration with the Blüthgen group of the Institute of Pathology (Charité - University Medicine Berlin). The principle is that a specific antibody coupled to a color-coded bead identifies the protein of interest while a second detection antibody determines the magnitude of a distinct phosphorylation. For this analysis, the Bio-Plex Pro Cell Signaling Reagent Kit (BIO-RAD) was used. The treated lymphoma cells were cooled down by addition of the three-fold volume of ice-cold DPBS supplemented with $1 \times$ PhosSTOP (Roche) and $100 \mu \mathrm{M}$ sodium orthovanadate. After 5 minutes centrifugation $\left(500 \times \mathrm{g}, 4^{\circ} \mathrm{C}\right)$ the cells were washed once. According to the instruction manual the cells were lysed in the provided buffer containing $1 \times$ factor $Q G$ and $2 \mathrm{mM}$ PMSF. After shaking for 20 minutes at $4^{\circ} \mathrm{C}$ debris was removed by centrifugation $\left(14000 \times \mathrm{g}, 4^{\circ} \mathrm{C}\right)$ for 15 minutes. The protein concentration was determined with the BCA Protein Assay Kit according to the user guide. In brief, the samples were diluted 1:8 and 1 part of solution B was mixed with 50 parts of solution A. $112 \mu \mathrm{l}$ of the solution mix was added to $14 \mu \mathrm{l}$ diluted sample, the samples were shaken for 30 seconds and then incubated for 30 minutes at $37^{\circ} \mathrm{C}$. To determine the protein concentration the absorption was measured at $560 \mathrm{~nm}$. Afterwards the samples were transferred in a 96 well plate, stored at $-80^{\circ} \mathrm{C}$ and shipped on dry ice to the Blüthgen group for analysis.

The analysis with the Bio-Plex Protein Array system (BIO-RAD) was done by Anja Sieber of the Institute of Pathology (Charité - University Medicine Berlin) as published before (Klinger et al., 2013) and according to the manufacturer's instructions. Specific beads were used for p-SYK (Y352), p-70 kDa zeta-chain associated protein (ZAP70) (Y319), p-BTK (Y223), p-AKT (S473), p-40S ribosomal protein S6 (RPS6) (S235/S236), p-Bcl2associated agonist of cell death (BAD) (S136), p-MEK1 (S217/S221), p-ERK1/2 (T202/Y204, T185/Y187), p-90 kDa ribosomal protein S6 kinase (p90RSK) (S380), p-GSK3ab (S21/S9), p-p38 MAPK (T180/Y182), p-HSP27 (S78), p-JNK (T183/Y185), p-c-Jun (S63) and p-p65 NF-KB (S536). The Bio-Plex manager software was used for data acquisition. Subsequently, Bertram Klinger of the Institute of Pathology (Charité - 
University Medicine Berlin) performed the evaluation and network modeling using Modular Response Analysis-based R package STASNet (Dorel et al., 2018; Klinger et al., 2013).

\subsubsection{Mass spectrometry based phosphoproteomics}

In order to ensure a complete incorporation of the isotope labeled amino acids, P493-6 cells were harvested after growing in cell labeling medium for 7 days. To that, 1 million cells were sedimented $\left(500 \times \mathrm{g}, 4^{\circ} \mathrm{C}\right)$ and washed once with DPBS. The cell pellet was resuspended in $500 \mu \mathrm{l}$ urea buffer and the protein concentration determined. An aliquot of each labeling was analyzed by mass spectrometry to ensure a protein labeling above $95 \%$ and to exclude an arginine to proline conversion.

For phosphoproteomics, cells were harvested by centrifugation $\left(500 \times \mathrm{g}, 4^{\circ} \mathrm{C}\right)$ for 5 minutes and washed once with ice-cold DPBS $+1 \times$ PhosSTOP (Roche). The cell pellet was stored by $-80^{\circ} \mathrm{C}$ until lysis. Afterwards the cell pellet was resuspended in $450 \mu \mathrm{l}$ modified NP-40 lysis buffer for 30 minutes at $4^{\circ} \mathrm{C}$. The cell debris was removed by 15 minutes centrifugation $\left(14000 \times \mathrm{g}, 4^{\circ} \mathrm{C}\right)$ and the supernatant collected. The light and heavy labeled samples were mixed in equal parts, the protein concentration was determined with Roti-Quant (see 2.3.1) and the samples stored at $-80^{\circ} \mathrm{C}$ until mass spectrometry analysis.

The further steps were executed by Jasmin Corso from the Bioanalytical Mass Spectrometry Group of Henning Urlaub (Max-Planck-Institute for Biophysical Chemistry, Goettingen) as described elsewhere (Corso et al., 2016). In summary, a global enrichment of phosphopeptides was done and analyzed with liquid chromatography-tandem mass spectrometry. Raw data were analyzed with the MaxQuant software (Max Planck Institute for Biochemistry, DE) (Cox and Mann, 2008) and further processed using the Perseus software (Max Planck Institute for Biochemistry, DE) (Deshmukh et al., 2015). Phosphorylation sites with a localization probability lower than 0.75 were removed and the logarithmic SILAC ratios displayed as described by Corso et al. (Corso et al., 2016).

\subsection{Molecular Biology}

\subsubsection{RNA isolation}

For RNA analysis, cells were harvested by centrifugation $\left(500 \times \mathrm{g}, 4^{\circ} \mathrm{C}\right)$ for 5 minutes and washed once with ice-cold DPBS. Total RNA was isolated from the cell pellets using the NucleoSpin RNA kit (Macherey-Nagel) and following the user manual. In brief, the cell pellet was lysed without $\beta$-mercaptoethanol, debris was removed and the RNA was 
loaded to a spin column. After digestion of DNA and several washing steps the RNA was eluted in $50 \mu \mathrm{l}$ RNase free $\mathrm{H}_{2} \mathrm{O}$. The concentration was determined with the spectrophotometer ND-1000.

\subsubsection{Reverse transcription}

The SuperScript II Reverse Transcriptase kit (Invitrogen) was used to generate complementary DNA (cDNA) from messenger RNA. First, $1 \mu \mathrm{g}$ RNA was filled up with RNase free water to a total volume of $10 \mu \mathrm{l}$. After adding $2 \mu \mathrm{l}$ random hexamer primers the samples were denaturated at $70^{\circ} \mathrm{C}$ for 10 minutes. Furthermore, samples were cooled down on ice, supplemented with $8 \mu \mathrm{l}$ master mix (Table 15) and the reverse transcription performed in a thermocycler following the program detailed in Table 16.

Table 15: Reverse transcriptase master mix

\begin{tabular}{ll}
\hline Substance & Amount \\
\hline First strand buffer (5x) & $4 \mu \mathrm{l}$ \\
DTT $(0.1 \mathrm{M})$ & $2 \mu \mathrm{l}$ \\
Super Script II RT & $1 \mu \mathrm{l}$ \\
dNTPs (10 mM) & $1 \mu \mathrm{l}$ \\
\hline
\end{tabular}

Table 16: Thermocycler program

\begin{tabular}{ll}
\hline Temperature & Cycle length \\
\hline $25^{\circ} \mathrm{C}$ & 10 minutes \\
$42^{\circ} \mathrm{C}$ & 60 minutes \\
$65^{\circ} \mathrm{C}$ & 10 minutes \\
$4^{\circ} \mathrm{C}$ & continuously \\
\hline
\end{tabular}

\subsubsection{Quantitative real-time polymerase chain reaction}

To analyze distinct gene expressions a SYBR green-based qRT-PCR was performed in 384 well plates using the $7900 \mathrm{HT}$ Fast Real-Time PCR System. SYBR green is a nucleic acid binding dye emitting a much higher green fluorescence upon intercalation with double stranded DNA compared to single strands (Schneeberger et al., 1995). As the amount of DNA rises exponentially with the number of amplification cycles, the fluorescence signal increases equally. Hence, the number of cycles crossing a fluorescence threshold is inversely proportional to the DNA amount and defined as CT-value. The comparison of CT-values allows a relative quantification of gene expression. For this purpose, $5.6 \mu \mathrm{l}$ SYBR green mix were supplemented with $0.3 \mu \mathrm{M}$ of each primer and filled up to $8 \mu \mathrm{l}$ as 
PCR master mix. The cDNA samples were diluted to $50 \mathrm{ng} / \mu \mathrm{l}$ and $10 \mathrm{ng}$ of cDNA added to the PCR master mix. After closing the well plate with an optical adhesive cover, the analysis was done following the qRT-PCR program shown in Table 17.

Table 17: qRT-PCR program

\begin{tabular}{lll}
\hline Temperature & Cycle length & Cycle amount \\
\hline $95^{\circ} \mathrm{C}$ & 15 minutes & \\
\hline $95^{\circ} \mathrm{C}$ & 15 seconds & $40 \times$ \\
$60^{\circ} \mathrm{C}$ & 1 minute & \\
\hline $95^{\circ} \mathrm{C}$ & 15 seconds & \\
$60^{\circ} \mathrm{C}$ & 15 seconds & \\
$95^{\circ} \mathrm{C}$ & 15 seconds & \\
\hline
\end{tabular}

The relative quantification of gene expression was determined using the software $A B I$ $7900 \mathrm{HT}$ SDS 2.4 and $\mathrm{ABI} R Q$ Manager 1.2.1. Thereby, normalization was firstly implemented to the internal housekeeper glyceraldehyde-3-phosphate dehydrogenase (GAPDH) $(\triangle \mathrm{CT})$ in order to compensate technical differences:

$$
\Delta C T=C T_{\text {gene }} \text { of interest }-C T_{\text {housekeeper }}
$$

The relative gene expression changes were calculated by reference to the untreated control $(\triangle \Delta C T)$ :

$$
\Delta \Delta C T=C T_{\text {treatment }}-C T_{\text {control }}
$$

As the number of cycles is inversely proportional to the DNA amount, the expression fold changes can be calculated as follows:

$$
\text { relative expression }=2^{-\triangle \Delta C T}
$$

\subsubsection{RNA sequencing}

RNA sequencing was conducted in three previous projects at the clinic for Hematology and medical Oncology (University Medical Centre, Goettingen). Hence, the implementation of the BL-2 analysis is described by Wolff et al. (Wolff et al., 2018). For P493-6 and ABC DLBCLs, the RNA analysis was performed as described by Maren Feist (Feist, 2016) and Annekatrin Arlt (Arlt, 2018). 


\subsection{Statistics and bioinformatical analyses}

Statistical and bioinformatical analyses of the multiplex immunoassay were performed by Bertram Klinger of the Institute of Pathology (Charité - University Medicine, Berlin).

For the phosphoproteomic experiment, phosphosites quantified in less than two replicates were excluded. Significance testing was done using the two-way ANOVA method and a two-stage step-up method of Benjamini, Krieger and Yekutieli to correct for multiple comparisons by a FDR threshold of 0.05 . The calculation was performed with $\log _{2}$-transformed ratios and GraphPad Prism 7. Therefore, phosphosites altered in two or more replicates, with p-value of less than 0.05 and $\log _{2}$ ratio above 0.58 (over $50 \%$ fold change) were deemed significantly changed. The network representation of changed protein phosphorylations was created with STRING (Szklarczyk et al., 2015). Furthermore, a gene set enrichment was performed with the online DAVID bioinformatics annotation tool (Huang da et al., 2009). Thereby, a functional annotation clustering for biological processes (GOTERM_BP_Fat) was used with an EASE score of 0.1 and a medium classification stringency. Due to a long list of gene ontology terms, REVIGO was used with default settings and allowed similarity of 0.5 for summarization and visualization (Supek et al., 2011). The changed protein phosphorylations were also used to predict possible kinase activities with a literature-based kinase-substrate library of the online Kinase Enrichment Analysis 2 (KEA2) tool (Lachmann and Ma'ayan, 2009). 


\section{Results}

The result section is divided in two chapters. At first, the interplay of BCR-related signaling pathways was investigated to generate a semi-quantitative network by complementation of a literature-based model with the experimental data. Thereafter, the combined stimulation of IL10R and TLR9 was examined for their impact on the phosphoproteome to investigate the synergistic effects on proliferation in more detail.

\subsection{Tonic and active BCR signaling contains several feedback loops}

In a previous analysis, our group applied the Boolean-Nested Effect Model framework to gene expression changes after pathway perturbations using the BL cell line BL-2 with a tonic or activated BCR signaling (Pirkl et al., 2016). The results provided a first unsupervised model of the distal BCR signaling nodes and a hypothesis explaining how downstream nodes of this pathway were affected. Nevertheless, this analysis could not fully elucidate the signaling network. For a better understanding and extension of the network structure, protein phosphorylation analyses of the BCR signaling were performed to uncover the interplay and feedback loops of downstream effector pathways.

For data consistency, we used BL-2 cells again and investigated pathway perturbations during a tonic or an activated BCR signaling. The tonic BCR signaling is next to c-MYC a hallmark of BLs and includes in particular the activation of the PI3K pathway (Schmitz et al., 2012; Srinivasan et al., 2009). However, a fully active BCR signaling can be achieved by stimulation of the receptor with antigens and in case of BL-2 with $\alpha-\operatorname{lgM}$. This crosslinking of the BCR leads to an additional activation of the MAPK, NFAT and NF-KB pathways (Hendriks et al., 2014). For the inhibition of these signal transmissions, the same inhibitors were used as in the previous study and some new ones included. Beside the inhibition of PI3Ka, $\beta, \delta$ (Ly294002), MEK1/2 (U0126), TAK1 (5'-7-Oxozeanol), p38 MAPK (SB203580), JNK (SP600125) and IKKa, $\beta$ (ACHP), the list was extended with

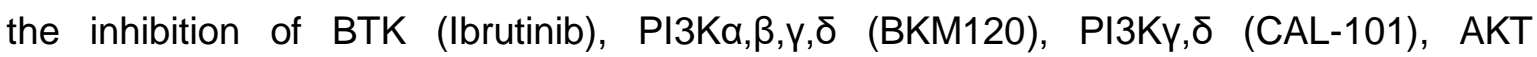
(MK-2206), mTOR (Rapamycin), MEK1/2 (AZD6244), JNK (JNK Inh VIII) and IKKa, $\beta$ (MLN120b). Instead of analyzing gene expression changes, we detected the pathway activations by measuring specific kinase phosphorylations. For this purpose, we used an antibody-based multiplex immunoassay to quantify 15 different protein phosphorylations simultaneously in one sample. Thereby, the phosphorylations of the upstream kinases SYK, ZAP70 and BTK as well as the pathway activations of PI3K-AKT, MEK-ERK, p38 MAPK, JNK and NF-KB were determined. Only such an approach allowed us to 
compare the thirteen inhibitor treatments during a tonic or active BCR signaling in BL-2 cells.

\subsubsection{Determination of a suitable time point for pathway interaction analyses}

As the kinetics of posttranslational modifications occur prior to the gene expression responses, the suitable time point to analyze protein phosphorylations had to be determined first. Therefore, protein phosphorylations of three major pathways were investigated after different durations of BCR activation. The phosphorylation of PI3K, AKT, pS6 kinase, MEK1, ERK1/2 and p38 MAPK was measured after 15, 30, 60 and 90 minutes of $\mathrm{BCR}$ stimulation with $\alpha$-IgM. The detection was done with immunoblot analysis (Figure $3 \mathrm{~A}$ ) and the multiplex immunoassay in BL-2 cells (Figure $3 \mathrm{~B}$ ).

A

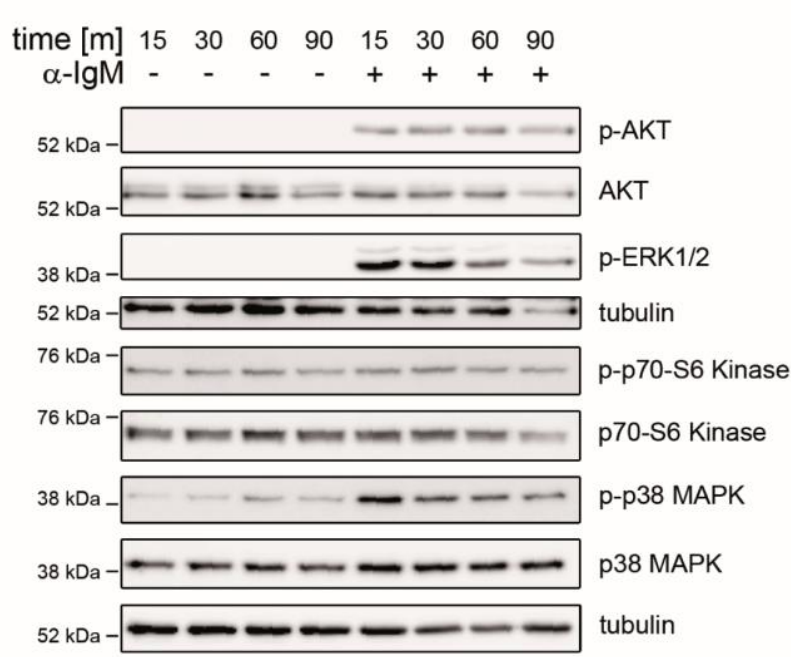

B
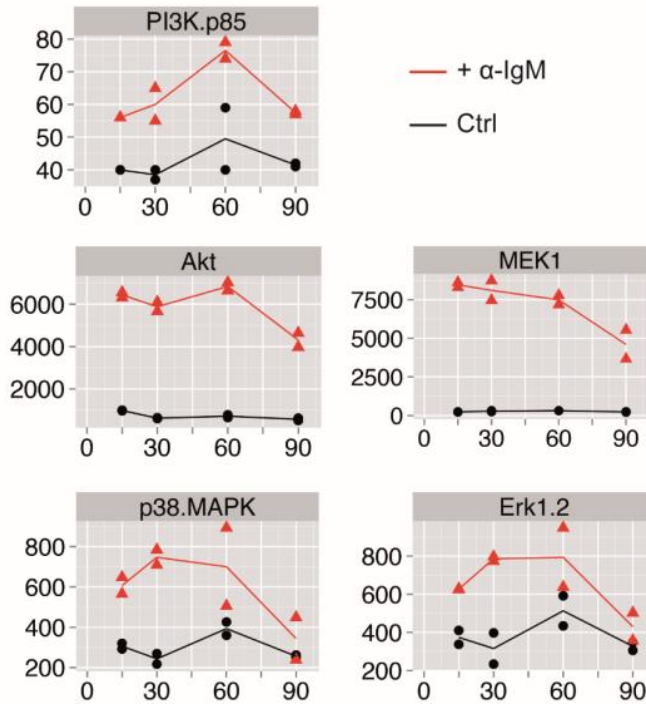

Figure 3: Time dependent activation of specific signaling pathways after BCR activation.

Protein phosphorylations were investigated after BCR activation by $\alpha-\operatorname{lgM}$ at the indicated time points. (A) Immunoblot analysis of AKT, ERK1/2, S6 kinase and p38 MAPK phosphorylation and total proteins. Tubulin served as loading control $(n=1)$. (B) multiplex immunoassay for quantitative phosphorylation analysis of PI3K, MEK1, AKT, ERK1/2 and p38 MAPK. Data points displayed the mean fluorescence intensity of two technical replicates. The black line displays the unstimulated control (Ctrl), whereas the red line indicates BCR activation by $\alpha$-IgM stimulation $(n=1)$.

Activation of the BCR led to an increasing phosphorylation of PI3K till 60 minutes in the multiplex immunoassay (Figure $3 \mathrm{~B}$ ). In addition, both analyses clearly showed a phosphorylation of the PI3K target AKT by $\alpha$-lgM stimulation up to 60 minutes (Figure $3 \mathrm{~A}, \mathrm{~B})$. After 90 minutes of BCR activation a declining phosphorylation was observed for $\mathrm{PI} 3 \mathrm{~K}$ and AKT. However, in the immunoblot analysis no phosphorylation changes were detected for the AKT downstream kinase S6 compared to the unstimulated control (Figure 3 A). The phosphorylation of MEK1, ERK1/2 and p38 MAPK was highest at 15 or 
30 minutes of $\alpha$-IgM stimulation, decreased slightly till 60 minutes and showed a stronger decline after 90 minutes (Figure $3 \mathrm{~A}, \mathrm{~B}$ ).

In conclusion, the PI3K activation increased up to 60 minutes of BCR activation, whereas the phosphorylations of the other kinases already decreased at this time point. In order to detect the most pathway activations and interactions, we decided to choose a time point suitable for all measured kinases. Therefore, 30 minutes of BCR activation was selected for further analysis.

\subsubsection{Tonic BCR signaling is enhanced by positive feedback loop}

We then analyzed the tonic BCR signaling in BL-2 cells with the multiplex immunoassay and the results are displayed in comparison to the untreated control in Figure 4. The quantitative values of this analysis are presented in the supplement (Figure A-1, Figure A2). In BL-2 cells it was shown that the inhibition of BTK, PI3K, AKT and mTOR lowered all measured protein phosphorylations except for p38 MAPK (Figure 4 A). In detail, the affected phosphorylations belonged to the upstream kinases SYK, ZAP70 and BTK as well as the pathway activations of PI3K-AKT, MEK-ERK, JNK, NF-KB and GSK3ß. As a signal inhibition of the PI3K pathway led to a decreased phosphorylation of downstream as well as upstream kinases, we hypothesized the existence of a positive feedback loop from mTOR to kinases nearby the BCR. However, the inhibition of MEK1/2 or p38 MAPK had nearly no effect on any detected phosphorylation. The impacts of JNK or NF-KB inhibition were also not notable and decreased only slightly the phosphorylations of SYK, ZAP70, BTK, GSK3 $\beta$ and the PI3K-AKT, MEK-ERK, JNK and NF-KB pathways. Therefore, no clear influences of the MAPKs or NF-KB on the other pathways were seen.

In order to confirm the obtained findings, a second $B L$ cell line was investigated using a smaller set of inhibitors. Similar results were obtained in BL-41 cells compared to BL-2 cells (Figure $4 \mathrm{~B}$ ). Only the MEK $1 / 2$ inhibition differed from the $\mathrm{BL}-2$ data and rather induced a slight increase of the phosphorylation of SYK, ZAP70, BTK, GSK3 $\beta$ and the PI3K-AKT, MEK-ERK, p38 MAPK, JNK and NF-kB pathways.

In summary, we concluded that the PI3K pathway enhances its own activation by a positive feedback loop to the proximal kinases of the BCR. In addition, the MAPKs and NF-KB pathways are not interacting with other pathways during the tonic BCR signaling. As the PI3K pathway is the main driver of the tonic BCR signaling (Sander et al., 2012; Schmitz et al., 2012), we suspected that the other pathways are maybe not active enough to reveal pathway interplays. 
A

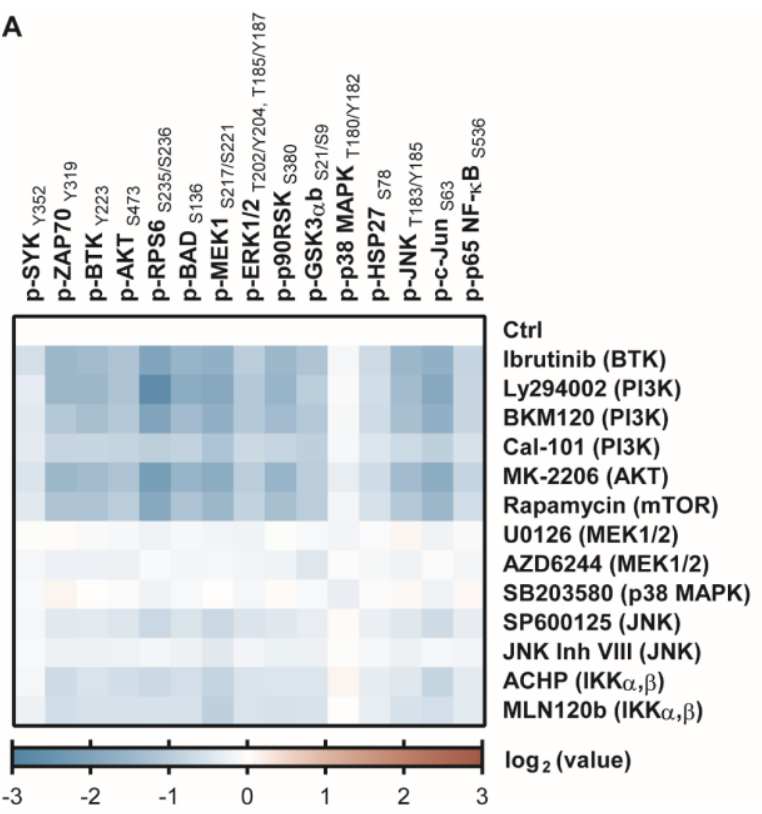

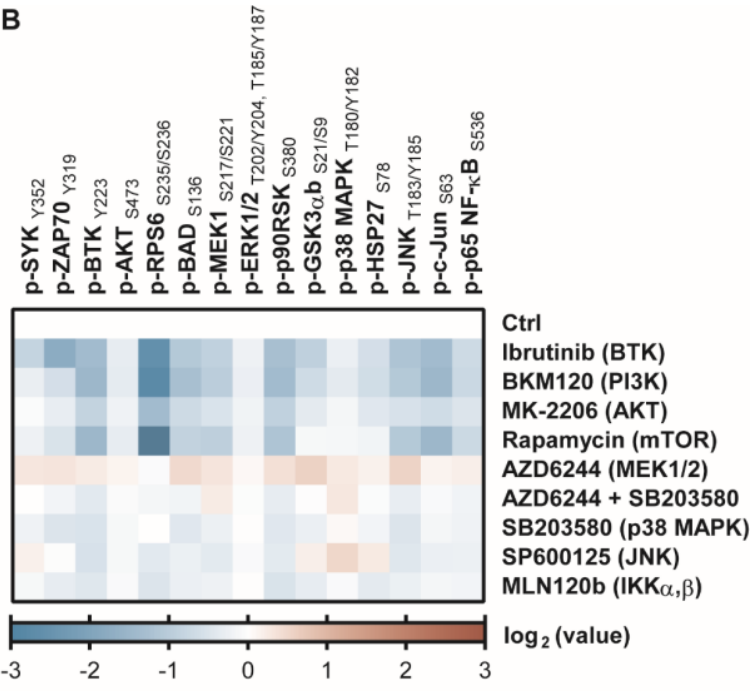

Figure 4: Signaling changes by pathway perturbations during the tonic BCR signaling.

Signal transduction was disrupted by inhibitors of BTK, PI3K, AKT, mTOR, MEK1/2, p38 MAPK, JNK and IKK,$\beta$ for 3.5 hours. Specific phosphorylations of SYK, ZAP70, BTK, AKT, RPS6, BAD, MEK1, ERK1/2, p90RSK, GSK3ab, p38 MAPK, HSP27, JNK, c-JUN and p65 NF-KB were measured with the multiplex immunoassay. The $\log _{2}$ values normalized to control are shown for the $B L$ cell lines BL-2 $(A)$ and BL-41 (B) $(n=3)$.

In order to determine whether all PI3K-AKT pathway inhibitors hamper the proliferation of BLs, the cell viability was examined over 72 hours. Besides, a MEK1/2 inhibitor was included to confirm that this pathway is not pivotal for the proliferation of BLs. While the inhibition of AKT and mTOR clearly reduced the viability of BL-2 cells to 72 hours, the $\mathrm{PI3K}$ and MEK1/2 inhibitor showed nearly no influences (Figure $5 \mathrm{~A}$ ). However, in BL-41 cells the inhibition of PI3K, AKT and mTOR decreased the cell viability, whereas only the MEK1/2 inhibition had no impact (Figure $5 \mathrm{~B}$ ). This indicated that the PI3K-AKT pathway was altogether important for BL proliferation.

We concluded that the PI3K is indeed the main driver of the tonic BCR signaling and the MEK-ERK pathway is negligible or not active under these conditions. 
A

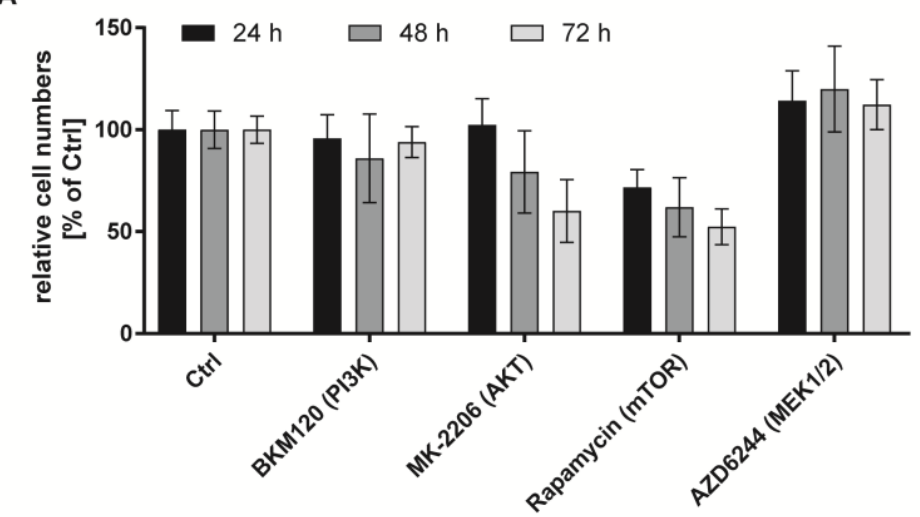

B

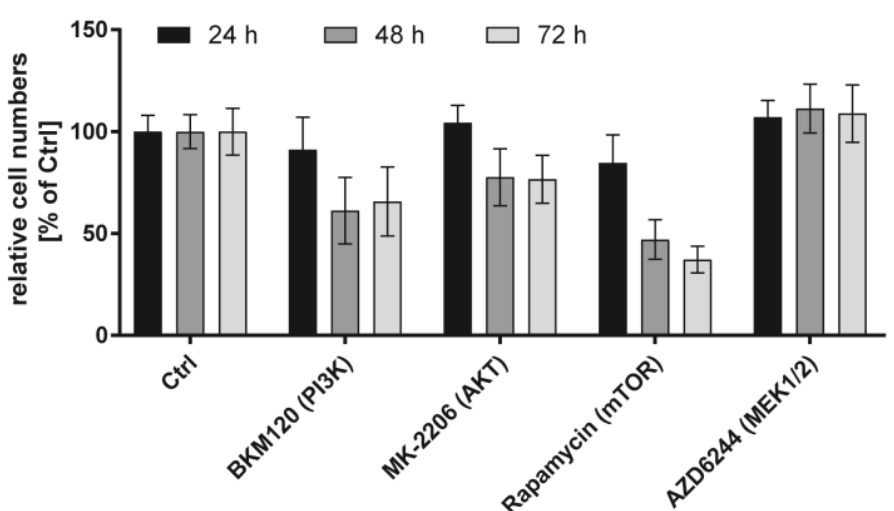

Figure 5: Cell viability of BLs after pathway inhibition during the tonic BCR signaling.

Cell viability assay was performed after 24,48 and 72 hours with the fluorescence dye Calcein AM. The cells were treated with inhibitors of PI3K, AKT, mTOR and MEK1/2. The amount of viable cells was normalized to control and the mean $+/-S D$ is displayed for the Burkitt lymphoma cell lines BL-2 (A) and BL-41 (B) $(n=3)$.

\subsubsection{Active BCR signaling is influenced by positive and negative interplays}

Next we examined the pathway phosphorylations after 30 minutes of BCR activation in BL-2 cells with the multiplex immunoassay. The quantitative values of this analysis are presented in the supplement (Figure A-1, Figure A-2). The a-IgM stimulation of the BCR increased all measured protein phosphorylations in comparison to the unstimulated control (Figure $6 \mathrm{~A}$ ). More precisely, the activation of SYK, ZAP70, BTK, GSK3 $\beta$ and the PI3K-AKT, MEK-ERK, p38 MAPK, JNK and NF-KB pathways were heightened.

To verify these findings, BL-41 cells were included as well (Figure 6 B). Similar to BL-2, the phosphorylations of SYK, ZAP70, BTK, GSK3 $\beta$ and the PI3K-AKT, MEK-ERK and $\mathrm{NF}-\mathrm{kB}$ pathways were stronger after $\alpha$-IgM stimulation compared to control cells. However, no phosphorylation changes were detected for p38 MAPK whereas the phosphorylation of the downstream target HSP27 was reduced. The JNK phosphorylation was not altered as well, while the phosphorylation of c-Jun was enhanced. As the exact 
signal transduction to the MAPKs was still not fully clarified, we suggested that little differences in the activation of upstream kinases were an explanation for these variations.

In conclusion, activation of the BCR receptor induced the phosphorylation of SYK, ZAP70 and BTK as well as a further activation of the PI3K-AKT, MAPK and NF-KB pathways as described previously (Hendriks et al., 2014). Besides, we assumed that the signal distribution to the MAPK pathways depend on the activity of upstream kinases and can differ between cell lines.

For an easier interpretation of the inhibitor effects, the values were normalized to the $\alpha$-IgM-stimulated control. Similar to the tonic BCR results, the inhibition of BTK, PI3K, AKT and mTOR diminished all measured protein phosphorylations in BL-2 cells (Figure $6 \mathrm{C}$ ). Thereby, the phosphorylations of the upstream kinases SYK, ZAP70 and BTK as well as the pathway activations of PI3K-AKT, MEK-ERK, p38 MAPK, JNK, NF-KB and GSK3 $\beta$ were affected. We concluded that the enhanced PI3K pathway after BCR activation also comprised a positive feedback loop from mTOR to the BCR.

In addition, a similar pattern like for PI3K inhibitors was seen after inhibition of MEK1/2 (Figure $6 \mathrm{C}$ ). The MEK1/2 inhibitor caused a lower phosphorylation of SYK, ZAP70, BTK, GSK3 $\beta$ and of the PI3K-AKT, p38 MAPK, JNK and NF-KB pathway, while its own phosphorylation was enhanced. As ERK1/2 is known to reduce its own pathway activity through inactivating phosphorylations of RAF-1 and MEK1/2 (Steelman et al., 2011), we suggested that the signal inhibition to ERK1/2 provoked the increase of the activating MEK1 phosphorylation. Furthermore, the inhibition of p38 MAPK heightened strongly the phosphorylation of MEK1 and ERK1/2. In comparison to the other pathways only slight increased phosphorylations of ZAP70, BTK, AKT, GSK3 $\beta$, JNK, c-Jun and NF-KB were detected. These pointed out that p38 MAPK attenuated especially the MEK-ERK pathway. Comparable with the tonic BCR signaling in BL-2 cells, JNK and NF-kB inhibition achieved only little phosphorylation changes. Thereby, the phosphorylations of SYK, ZAP70, BTK, GSK3 $\beta$ and the PI3K-AKT, MEK-ERK, JNK and NF-KB pathways were only slightly diminished. Thus, no clear influences of JNK and NF-KB were seen on the other pathways after BCR activation. 
A

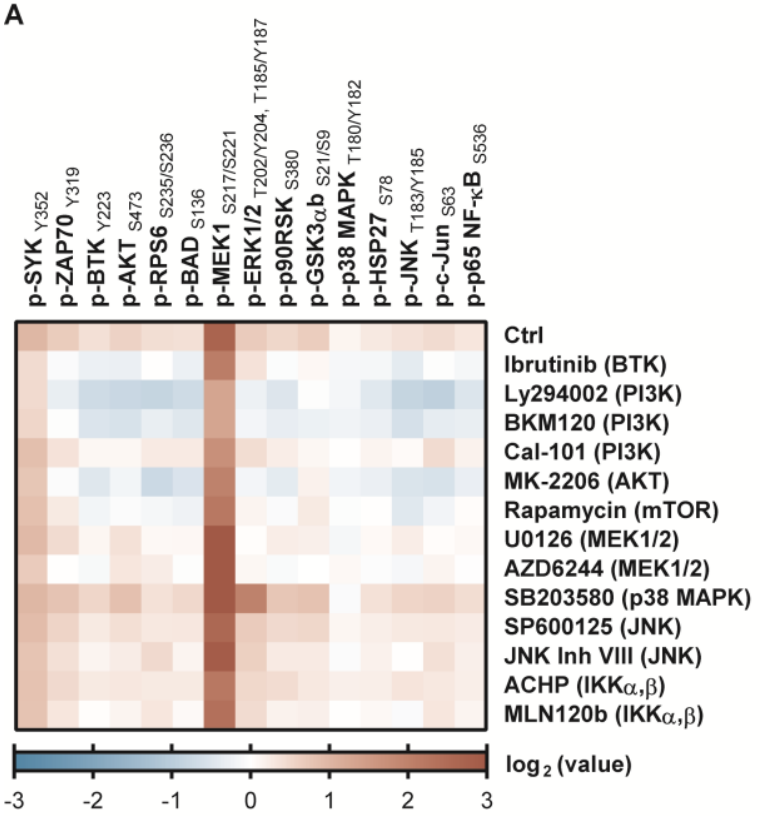

C

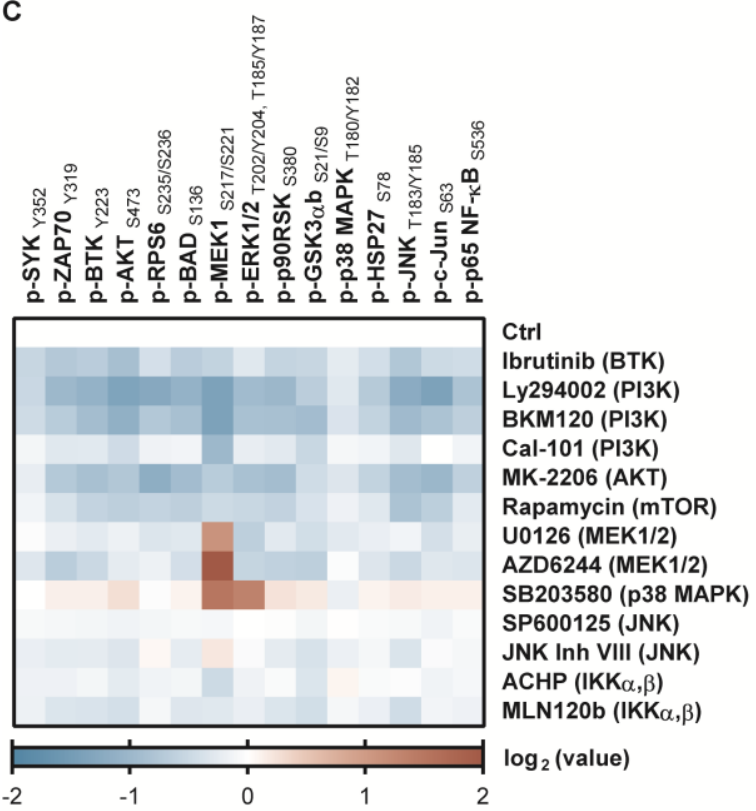

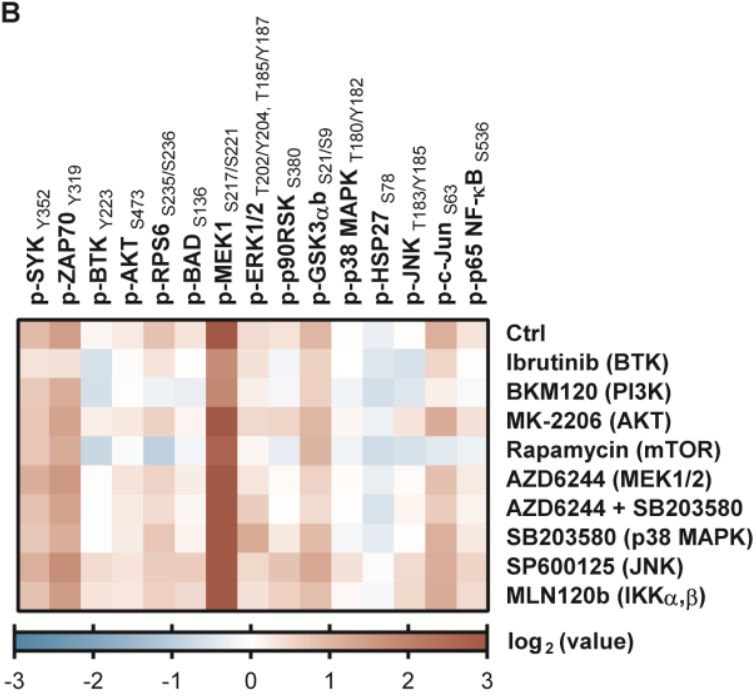

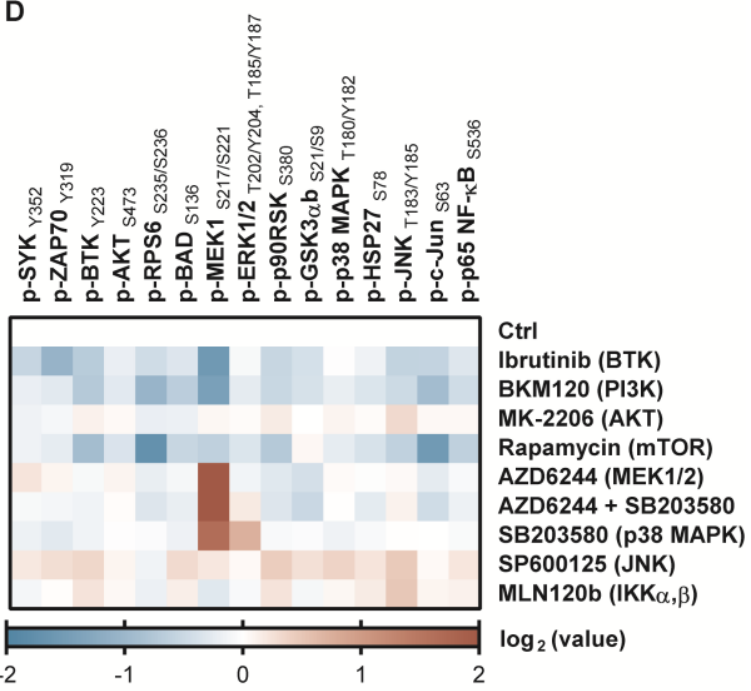

Figure 6: Signaling changes by pathway perturbations during the active BCR signaling.

The signal transduction was disrupted by inhibitors of BTK, PI3K, AKT, mTOR, MEK1/2, p38 MAPK, JNK and IKK $\alpha, \beta$ for 3 hours. Afterwards the BCR were activated by $\alpha$-IgM for 30 minutes. Specific phosphorylations of SYK, ZAP70, BTK, AKT, RPS6, BAD, MEK1, ERK1/2, p90RSK, GSK3ab, p38 MAPK, HSP27, JNK, c-JUN and p65 NF-KB were measured with the multiplex immunoassay. The $\log _{2}$ values normalized to untreated control are shown for the Burkitt lymphoma cell line BL-2 (A) and BL-41(B). Furthermore, the $\log _{2}$ values of $\mathbf{A}$ and $\mathbf{B}$ were normalized to the $\alpha$-IgM-stimulated control and displayed for BL-2 (C) and BL-41 (D) $(n=3)$.

For the verification of these results, a second $B L$ cell line was examined with a smaller set of inhibitors. The BL-41 cells showed similar results in comparison with BL-2 cells (Figure $6 \mathrm{D})$. Therefore, only the differences are mentioned in the following. In contrast to the BL-2 results, the inhibition of AKT caused a stronger phosphorylation of p90RSK and JNK while the other kinase phosphorylations were not affected compared to the antigen-stimulated 
control. In addition, the JNK inhibitor showing no effects in BL-2 led to increased phosphorylation of SYK, ZAP70, BTK, GSK3 $\beta$ and the PI3K-AKT, MEK-ERK, p38 MAPK, JNK and NF-KB pathways. After inhibition of NF-KB, the increased phosphorylation of BTK, p90RSK, HSP27 and JNK was contrary to the reduced phosphorylations in BL-2 cells. Another difference compared to the BL-2 analysis was the combined inhibition of MEK1/2 and p38 MAPK. As a negative impact from p38 MAPK was considered on MEK1 and ERK1/2, the inhibition of both kinases was performed to reveal whether p38 MAPK directly influences ERK1/2. The phosphorylation of ERK $1 / 2$ was over $80 \%$ lower after inhibition of p38 MAPK and MEK1/2 compared to the p38 MAPK inhibition alone. This pointed out that p38 MAPK reduced mainly the phosphorylation of MEK1 or upstream components.

In summary, the PI3K pathway was also promoted by a positive feedback loop to proximal kinases of the BCR after receptor activation. Furthermore, the BCR activation induced negative feedbacks on the MEK-ERK pathway which are mediated by ERK1/2 and p38 MAPK on upstream kinases of ERK1/2.

\subsubsection{Schematic model represents interactions of signaling pathways}

Current literature-based networks present mostly a linear signal transduction from the receptor along the pathway components. Furthermore, the data collected from different publications often focus on a particular signaling or on protein complexes within the own pathway. For the BCR signaling, the proximal events are so far investigated in more detail (Satpathy et al., 2015), even though a final network is still missing. In contrast, the distal signaling of the BCR is just partially understood. In order to refine the network structure, we used a literature-derived signaling network, depicted in a recent review, as a starting point (Figure A-3) (Hendriks et al., 2014). In collaboration with Bertram Klinger (Institute of Pathology, Charité - University Medicine Berlin), a semi-quantitative network analysis was performed on the perturbation data using the modular response analysis and the profile likelihood (Klinger et al., 2013). For this purpose, pathway interactions were included or excluded to achieve the best likelihood and network.

In Figure 7 the network for the BL-2 analysis is displayed. As the network analysis of BL-41 is comparable, it is presented in the appendix (Figure A-4). After BCR activation, the signal was transmitted through SYK, BTK and PI3K over AKT to mTOR (upper part of Figure 7). Furthermore, a link from mTORC1 to GSK3 $\beta$ and the BCR was proposed. The interaction between AKT and GSK3 $\beta$ was known before and confirmed by our analysis (Baracho et al., 2011). While negative feedbacks were only described for the PI3K-AKT 
pathway (Logue and Morrison, 2012), our results suggested a positive feedback loop from mTOR to the BCR in BLs.

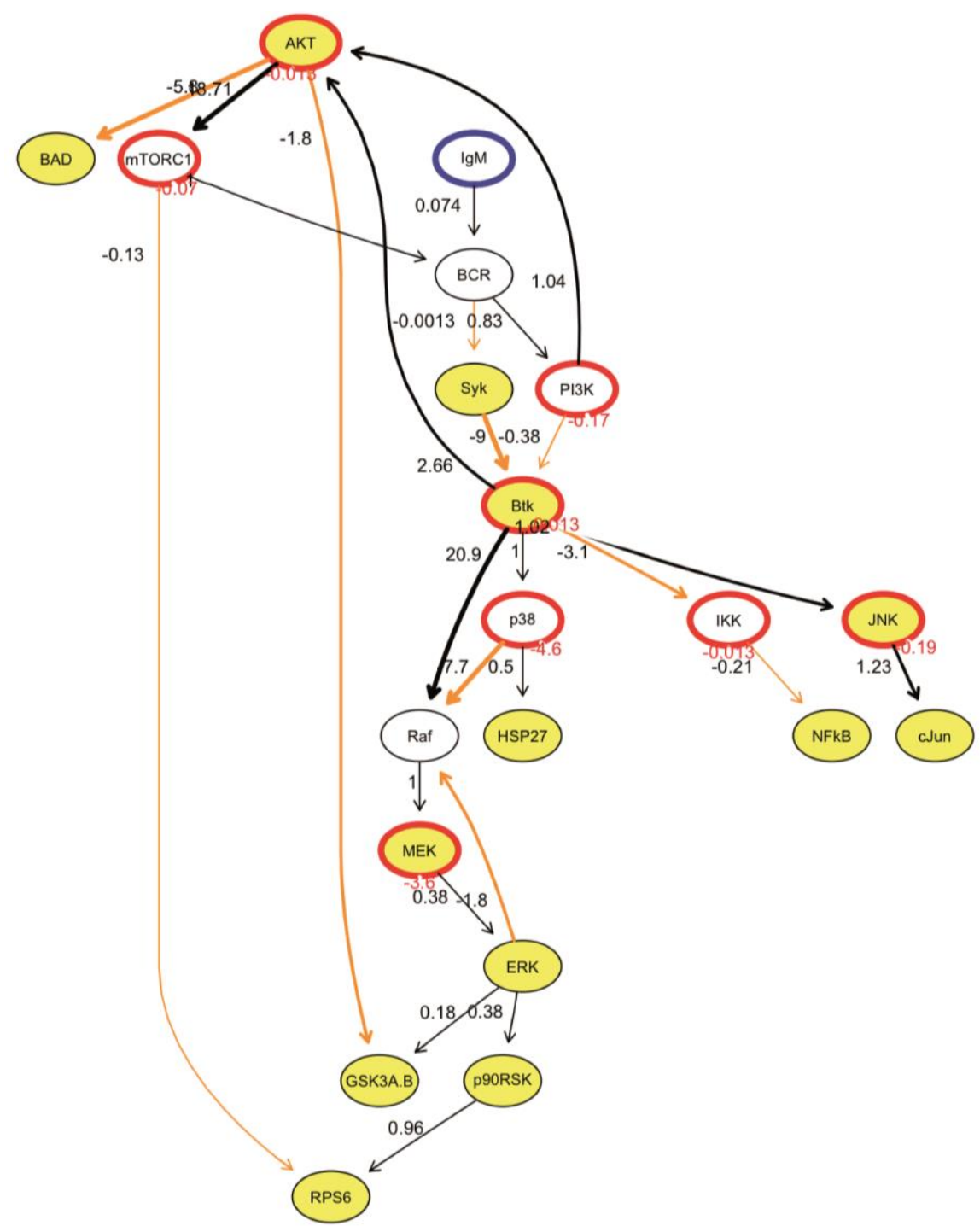

Figure 7: Network model of BCR signaling interactions and feedbacks.

Network model of BL-2 based on Modular Response Analysis by STASTNet was calculated by Bertram Klinger (Institute of Pathology, Charité - University Medicine Berlin). Stimulated (blue), inhibited (red) and measured (Yellow) nodes are displayed. The corresponding numbers reveal the inhibition strength (red) and the value of the local response coefficients (black). Arrows present interaction with positive (black) or negative (orange) local response coefficients.

The BCR signal was also transduced through SYK, PI3K and BTK to RAF-1, p38 MAPK, JNK and IKK (lower part of Figure 7). The activation of JNK led to the phosphorylation of c-Jun, whereas IKK subsequently activated NF-KB. For these both pathways, no feedbacks or other interactions were likely in our analyses. Besides, p38 MAPK induced HSP27 and influenced RAF-1. Although our results just implicated that p38 MAPK attenuates the MEK-ERK pathway upstream of ERK1/2, a connection from p38 MAPK to RAF-1 was assumed based on the literature. Finally, the activation of RAF-1 mediated the 
phosphorylation of MEK1 and further ERK1/2. The activated ERK1/2 reduced then the activation of RAF-1 and GSK3 $\beta$. Therefore, our findings confirmed the negative feedback from ERK1/2 to RAF-1 and validated the interaction with GSK3 $\beta$ in BLs.

To sum up, our signaling analyses revealed a positive feedback loop of the PI3K-AKT pathway which has not been described before. In addition, two negative feedbacks on the MEK-ERK pathway were mediated by ERK1/2 and p38 MAPK. The phosphorylation of GSK3 $\beta$ was both AKT- and ERK1/2-dependent. Therefore, the pathway analyses contribute to the validation of known feedbacks, to the discovery of new interactions and to a better understanding of the pathway interplays in BL cell lines.

\section{2 p38 MAPK attenuates the MEK-ERK pathway}

The importance of the PI3K pathway for BL proliferation is well studied (Sander et al., 2012; Schmitz et al., 2012) while the role of MAPKs is not elucidated and less is known about the attenuation of the MEK-ERK pathway. So far the capability of ERK1/2 to inhibit MEK1/2 and RAF-1 has been discovered (Dougherty et al., 2005). An influence on the MEK-ERK pathway by p38 MAPK was only seen in other cell types and controversially discussed (Birkenkamp et al., 2000; Zhang et al., 2003). Therefore, we focused on the negative regulation of the MEK-ERK pathway in our next analyses.

\subsection{1 p38a (MAPK14) limits the MEK-ERK pathway}

As the RAF-1 activity increased linearly by raising concentration of the p38 MAPK inhibitor SB203580 above the required inhibitor concentrations to block p38 MAPK, this effect was referred to an unspecific inhibitory effect (Kalmes et al., 1999). However, an increased MEK-ERK signaling after p38 inhibition was also observed with another inhibitor (Hirosawa et al., 2009) and with dominant negative p38 MAPK expression (Aguirre-Ghiso et al., 2001). Thus, we validated the p38 MAPK influence on the MEK-ERK pathway in BL- 2 cells first. As shown in Figure $8 A$, the phosphorylation of MEK1 and ERK1/2 was induced by antigen stimulation. Additionally, the inhibition of p38 MAPK (SB203580) led to much higher levels of MEK1 and ERK1/2 phosphorylations compared to the antigenstimulated control. To confirm this effect, p38 MAPK was downregulated with specific siRNAs. p38 MAPK comprises four subunits and the p38 inhibitor SB203580 is known to block the $\alpha$ - and $\beta$-subunit (Bain et al., 2007). Therefore, siRNAs for p38 (MAPK14) were chosen as the $\beta$-subunit is only expressed to a small extent in BL-2 cells (Figure $8 \mathrm{C}$ ). The immunoblot analysis (Figure $8 \mathrm{~B}$ ) and the summary of three independent siRNA transfections (Figure $8 \mathrm{D}$ ) revealed that a downregulation of p38a induced two-fold higher 
ERK1/2 phosphorylations compared to the antigen-stimulated control. We concluded that the p38 $\alpha$ (MAPK14) subunit was involved in the attenuation of the MEK-ERK pathway and that the effect was not an inhibitory side effect.

A

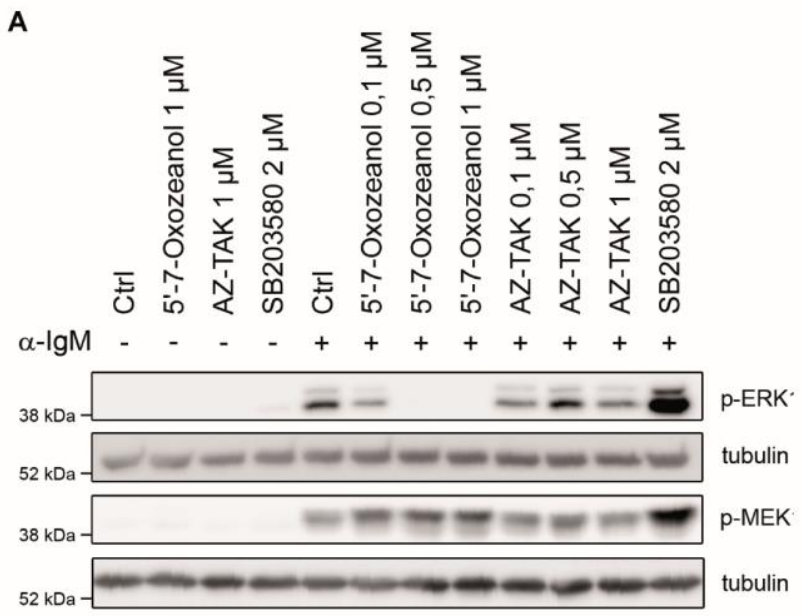

B

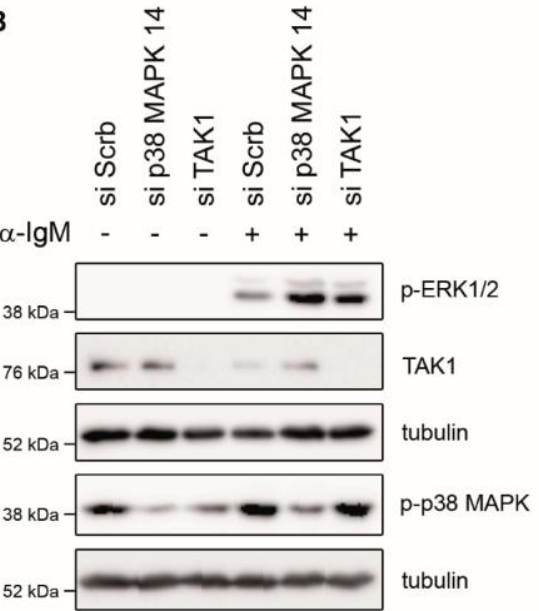

C

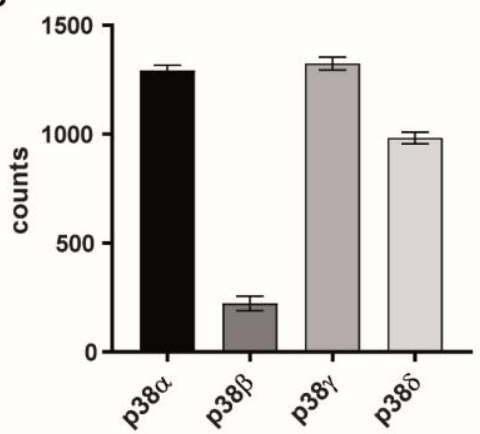

D

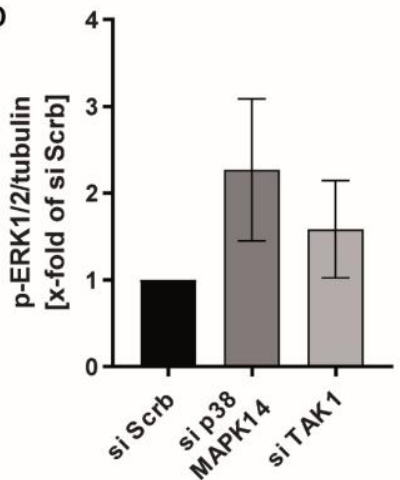

Figure 8: Verification of TAK-1 and p38 MAPK influence on the MEK-ERK pathway

(A) Representative immunoblot analysis of TAK-1 (5'-7-Oxozeanol, AZ-TAK) or p38 MAPK (SB203580) inhibition in BL-2 cells. Kinases were inhibited for 3 hours and the BCR additionally activated for 30 minutes with $\alpha-\operatorname{lgM}$. Tubulin served as loading control $(n=2)$. (B) Representative immunoblot analysis of TAK-1 and p38a (MAPK14) knockdowns with specific siRNAs. Protein phosphorylation was measured after 24 hours of transfection and 30 minutes $\alpha$-lgM stimulation. Tubulin served as loading control $(n=3)$. (C) Total reads of the p38 subunits a (MAPK14), $\beta$ (MAPK11), Y (MAPK12), $\delta$ (MAPK13) are displayed from RNA sequencing analysis of BL-2 cells $(n=3)$. (D) Quantification of phosphorylated ERK1/2 of the immunoblot analyses from $B$.

Besides, a second effect of the p38 MAPK activating kinase TAK-1 was observed. Inhibition of TAK-1 led to an increased MEK1 but not ERK1/2 phosphorylation (data not shown) and was more similar to a MEK1/2 than to a p38 MAPK inhibition. As an upstream activation of p38 MAPK was expected to obtain comparable results, the role of TAK-1 on ERK1/2 was analyzed. As shown in Figure 8 A, inhibition of TAK-1 with 5'-7-Oxozeanol caused a slight increase of MEK1 phosphorylation and a diminished ERK1/2 phosphorylation after BCR activation. However, an additional TAK-1 inhibitor (AZ-TAK) did not show comparable results. In addition, downregulation of TAK-1 with specific 
siRNAs could also not confirm the effects of 5'-7-Oxozeanol (Figure 8 B,D). We suggested that the effect of TAK-1 inhibition on MEK1 and ERK1/2 was an inhibitor specificity and hence 5'-7-Oxozeanol was excluded from the analyses.

\subsubsection{RAF-1 phosphorylations are affected by ERK1/2 but not by p38 MAPK}

To investigate whether the p38 MAPK effect on the MEK-ERK pathway occurred directly after BCR activation, an earlier time point was chosen. Apart from 30 minutes of $\alpha$ - IgM stimulation, the ERK phosphorylation was analyzed after 5 minutes of BCR activation (Figure $9 \mathrm{~A}$ ). In all three tested cell lines the ERK1/2 phosphorylation was higher at 5 minutes of $\alpha$-IgM stimulation compared to 30 minutes. Additionally, inhibition of p38 MAPK (SB203580) caused a stronger increased of phosphorylated ERK1/2 at any time point compared to the antigen stimulated control. Therefore, we assumed that p38 MAPK attenuated directly or through phosphatases the MEK-ERK pathway although transcriptional changes by p38 MAPK inhibition were not excluded.

As purified p38 MAPK was shown to inhibit RAF-1 activity (Hutchison, 2012), we further investigated the phosphorylations of RAF-1. Over 50 phosphorylation sites were described for RAF-1. However, one study suggested that the activating phosphorylation on serine 338 of RAF-1 was heightened by p38 MAPK inhibition (Zhang et al., 2003). A slight phosphorylation of RAF-1 on serine 338 was detectable in BL-2 and BL-41 cells after BCR activation (Figure $9 \mathrm{~A}$ ). The stimulation with $\alpha$-IgM for 5 or 30 minutes showed no differences and no additional influences were shown on this activating phosphorylation of RAF-1 by p38 MAPK inhibition (SB203580).

Apart from many activating phosphorylations, the phosphorylations of serine 289, 296 and 301 are described to induce an inactive conformation of RAF-1. This inactive conformation is enhanced by active ERK1/2 and known as negative feedback for the MEK-ERK pathway (Dougherty et al., 2005). We further examined whether this inactive conformation was promoted by p38 MAPK (Figure $9 \mathrm{~A}$ ). While an increase of these phosphorylations was measured up to 30 minutes after $\alpha$-lgM stimulation, the inhibition of p38 MAPK (SB203580) had no effect. Therefore, we tested whether the inactive conformation of RAF-1 was ERK1/2 dependent or only induced by BCR activation (Figure 9 B,C). After 30 minutes of $\alpha$-IgM stimulation, the inactive phosphorylations of RAF-1 were clearly detectable in BL-41 cells and diminished by MEK1/2 inhibition (AZD6244). As before, no impacts on the ERK-dependent phosphorylation of RAF-1 were found by p38 MAPK inhibition. 
This indicated that p38 MAPK attenuates the MEK-ERK pathway directly after BCR activation while the negative feedback from ERK to RAF-1 increases up to 30 minutes. Furthermore, p38 MAPK had no impacts on the activating serine 338 or the inactivating serine 289, 296 and 301 phosphorylations of RAF-1. The interaction of p38 MAPK to the MEK-ERK pathway and the involvement of RAF-1 requires further investigations.

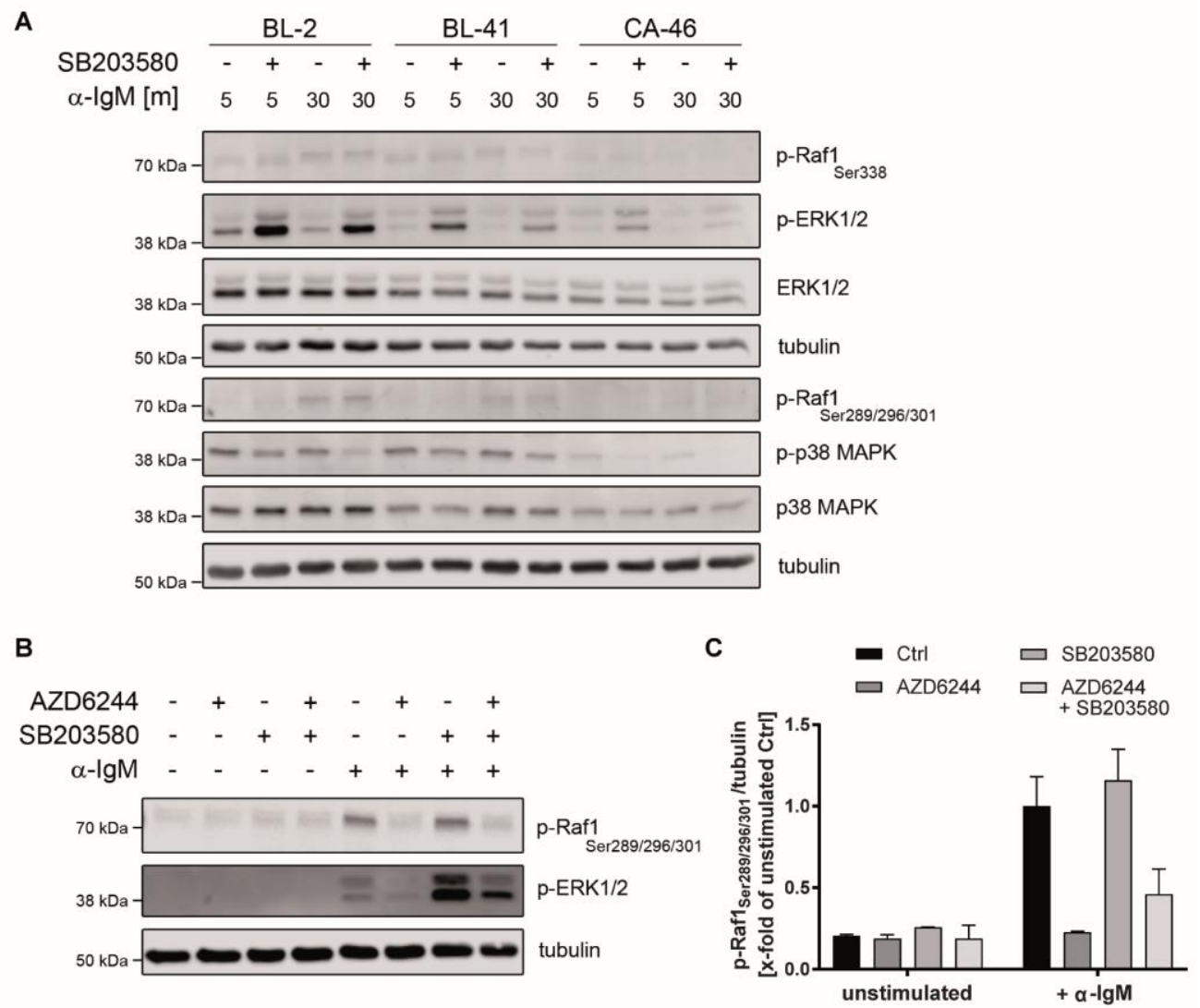

Figure 9: p38 MAPK and ERK1/2 impact on RAF-1 phosphorylations

(A) Representative immunoblot analysis of 3 hours p38 MAPK (SB203580) inhibition in the BL cell lines BL-2, BL-41 and CA-46. The BCR was stimulated with $\alpha$-IgM for 5 and 30 minutes. Tubulin served as loading control and imaging was done with fluorescence antibodies $(n=2)$. (B) Representative immunoblot analysis of MEK1/2 (AZD6244) and p38 MAPK (SB203580) inhibition in BL-41. After 3 hours inhibitor treatment, the BCR was activated with $\alpha$-lgM for 30 minutes. Tubulin served as loading control and imaging was done with fluorescence antibodies $(n=2)$. (C) Quantification of the inactivating RAF-1 phosphorylations of the immunoblot analyses from $B$.

\subsection{3 p38 MAPK reduces activation and duration of the MEK-ERK pathway}

Beside the connection of p38 MAPK to the MEK-ERK pathway, the question was also whether p38 MAPK limited the ERK1/2 activity. Firstly, the signaling duration of ERK1/2 was investigated more precisely. Once activated, the ERK1/2 signal diminished during the first 5 to 30 minutes but was still present over hours (data not shown). Therefore, an 
experiment was planned whereby the BCR was activated for 5 minutes following a signal disruption by inhibition of the upstream kinase BTK. This analysis enabled us to monitor and compare the dephosphorylation of ERK1/2 between control and p38 MAPK inhibited BL-2 cells at several time points (Figure $10 \mathrm{~A}$ ). After 5 minutes of $\alpha$-lgM stimulation ERK $1 / 2$ was phosphorylated and this signal started to decline after 10 minutes of BTK inhibition by ibrutinib. In comparison to the respective control, inhibition of p38 MAPK (SB203580) caused stronger ERK1/2 phosphorylations at each time point. However, the dephosphorylation of ERK1/2 was not prevented and only delayed by p38 MAPK inhibition.

As the dephosphorylation did not provide sufficient insights into ERK1/2 activity, ERK1/2 was immune-precipitated and the kinase activity measured in BL-2 cells (data of our collaboration partner and not shown). After stimulation of the BCR with $\alpha$-lgM for 30 minutes, the ERK1/2 activity was about two-fold higher in p38 inhibited cells. Furthermore, in vitro stimulation of purified ERK1/2 revealed that the p38 MAPK inhibitor (SB203580) alone had no influence on the activity. In conclusion, p38 MAPK caused a reduced kinase activity of ERK1/2 and an unspecific inhibitor effect on ERK1/2 was excluded.

In order to evaluate whether the increased ERK1/2 activity is limited to cytosolic functions or could also affect nuclear tasks, the translocation of ERK1/2 was investigated in CA-46 cells (Figure $10 \mathrm{~B}$ ). The stimulation of the $\mathrm{BCR}$ with $\alpha-\lg M$ for 5 minutes led to phosphorylation and translocation of ERK1/2 to the nucleus. In comparison, a higher amount of phosphorylated ERK1/2 was detected in the nucleus after inhibition of p38 MAPK (SB203580). Nevertheless, the ERK1/2 phosphorylation was also increased in the cytosolic fraction after p38 MAPK inhibition in comparison to the antigen-stimulated control. We assumed that p38 MAPK could alter cytosolic as well as nuclear tasks of ERK1/2.

To sum up, p38 MAPK not only diminished the phosphorylation of MEK1 and ERK1/2 after BCR activation but also reduced the pathway activity. We concluded that p38 MAPK shortens the signal duration and activity of ERK1/2 thus influencing ERK targets. 
A Ibrutinib [m] $0 \begin{array}{llllllllll}0 & 5 & 10 & 20 & 30 & 0 & 5 & 10 & 20 & 30\end{array}$

SB203580 - - - - - + + + + +

$\alpha-\lg M++++++++++$

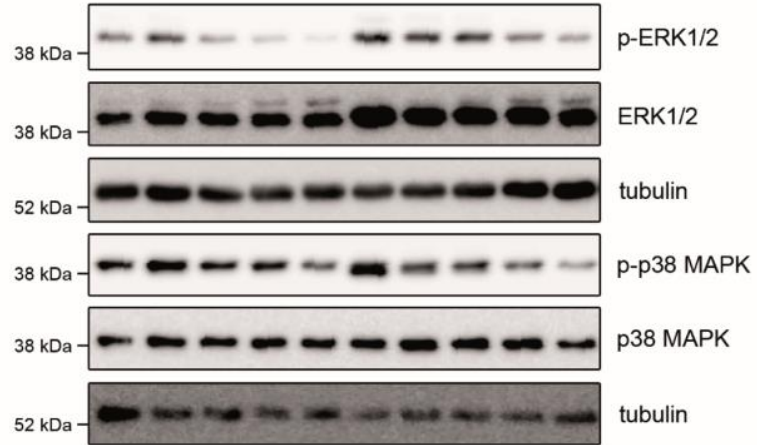

B

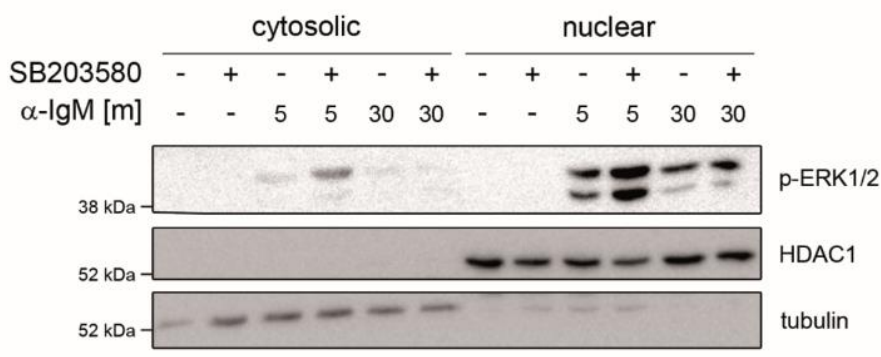

Figure 10: Alteration of ERK1/2 activity after p38 MAPK inhibition

(A) Representative immunoblot analysis of 3 hours p38 MAPK (SB203580) inhibition in BL-2 cells. The BCR was activated for 5 minutes by $\alpha$-IgM stimulation followed by a signal disruption through inhibition of the BCR-related kinase BTK for the indicated time points. Tubulin served as loading control $(n=3)$. (B) Representative immunoblot analysis of nuclear and cytosolic fractions after p38 MAPK (SB203580) inhibition in CA-46. After 3 hours inhibitor treatment the BCR was stimulated with $\alpha$-IgM for 5 and 30 minutes. HDAC1 and tubulin served as loading controls $(n=3)$.

\subsubsection{Early target gene expression of ERK1/2 is not influenced by p38 MAPK}

To examine whether ERK1/2 targets were affected by p38 MAPK, we investigated the expression of well-known target genes. The expression of several genes is mediated by ERK1/2 through binding to promotor regions or through activation of transcription factors (Fukunaga and Hunter, 1997; Shaul and Seger, 2007). We analyzed the target genes FOS, early growth response 2 (EGR2) and early growth response 3 (EGR3) because ERK1/2 can bind to the promotor region and quickly induces their expression (Yasuda et al., 2008). The activation of the BCR with $\alpha$-lgM for 3 hours strongly increased the expression of EGR2, EGR3 and FOS in BL-2 and CA-46 compared to the unstimulated control (Figure 11). In addition, all three gene expressions were ERK-dependent and diminished by inhibition of MEK1/2 (AZD6244, U0126). The p38 MAPK inhibition (SB203580) did not affect the gene expression compared to the antigen-stimulated control in BL-2 cells (Figure $11 \mathrm{~A}-\mathrm{C}$ ). In CA-46 cells the expression of FOS was also not altered by p38 MAPK inhibition (Figure 11 F). However, a slight increase was detectable for 
EGR2 and EGR3 expression after p38 MAPK inhibition compared to the antigenstimulated control in CA-46 (Figure 11 D-E). Although EGR2, EGR3 and FOS expression was induced by ERK1/2, the higher activity of ERK1/2 after p38 MAPK inhibition only led to a stronger expression of EGR2 and EGR3 in CA-46 cells.
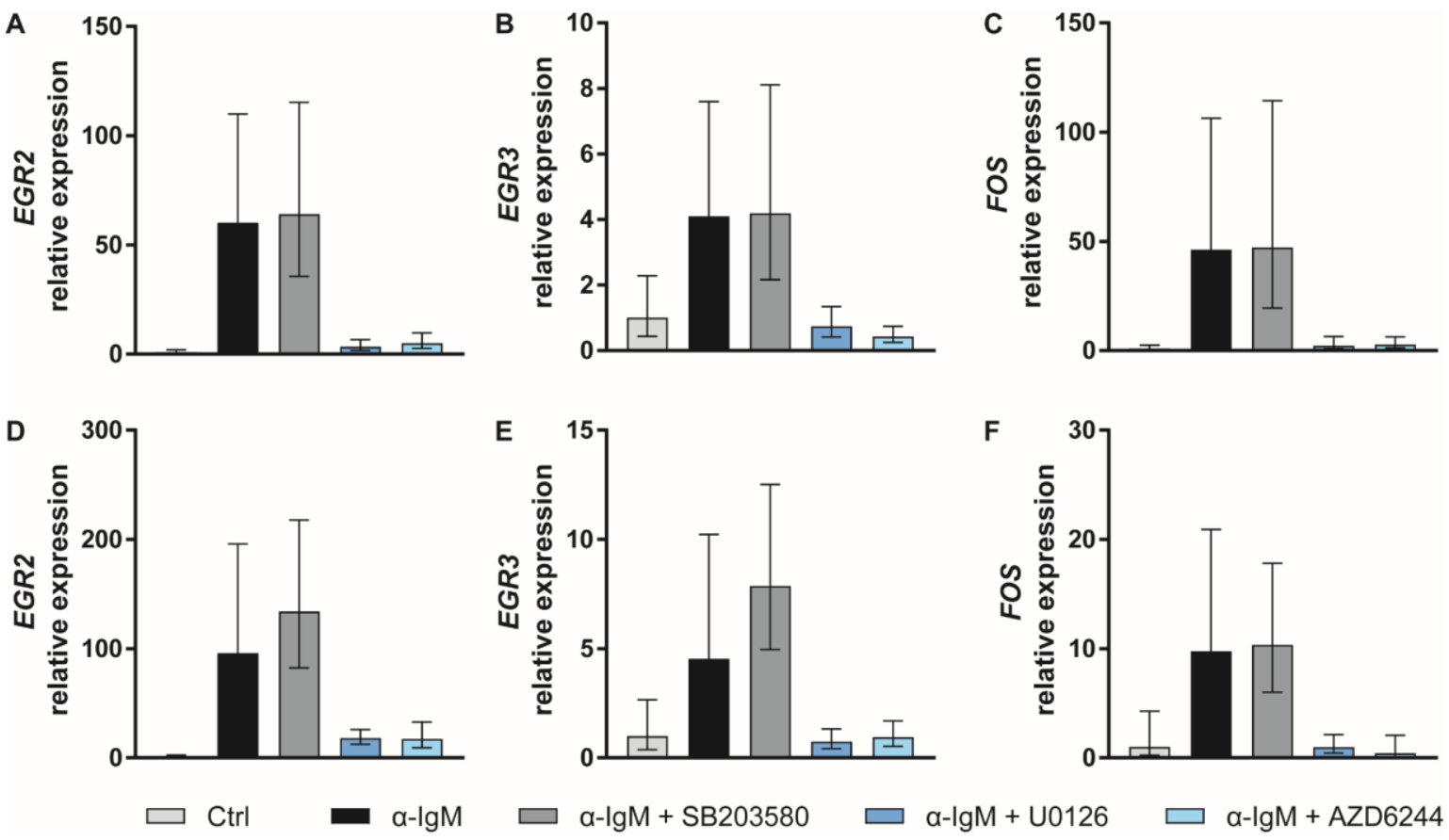

Figure 11: Impact on early target gene expressions by ERK1/2 and p38 MAPK

Gene expression analyses of early target genes were investigated after 3 hours inhibitor treatment with additionally 3 hours BCR activation. Data were normalized to GAPDH (housekeeper) and the untreated control. The mean +/- SD is shown for EGR2 (A, D), EGR3 (B, E) and FOS (C, F) for the $\mathrm{BL}$ cell lines BL-2 (A - C) or CA-46 (D - F) $(n=3)$.

In order to reduce the possibility of feedbacks or secondary effects that influence or limit the target gene expressions after 3 hours of BCR activation, the expression of EGR2 and EGR3 was analyzed after 1 hour of BCR activation (Figure 12). Due to the observed differences between BL-2 and CA-46 cells, a third lymphoma cell line, BL-41, was included. In all three lymphoma cell lines the stimulation with $\alpha$-lgM for 1 hour increased the expression of EGR2 and EGR3. Furthermore, the target gene expressions were ERK1/2-dependent (AZD6244, U0126) in all three cell lines. Unlike as before, the p38 MAPK inhibition (SB203580) resulted in a reduced expression of EGR2 and EGR3 compared to the antigen-stimulated control in BL-41 and CA-46 (Figure 12 B-C,E-F), while almost no difference was found in BL-2 cells (Figure 12 A,D).

In conclusion, EGR2, EGR3 and FOS are ERK1/2 target genes. However, the limiting effect of p38 MAPK on the EGR2 and EGR3 expression in CA-46 cells after 3 hours of BCR activation could not been verified after 1 hour of BCR activation. Interestingly, the 
fold changes revealed that the EGR2 expression increased up to 3 hours of $\mathrm{BCR}$ activation (Figure $11 \mathrm{~A}, \mathrm{D}$; Figure $12 \mathrm{~A}, \mathrm{C}$ ), whereas the EGR3 expression seemed to be terminated after 3 hours of BCR activation (Figure 11 A,D; Figure 12 A,C). Therefore, we suggested that p38 MAPK is not limiting the EGR2 expression and due to a peaking expression at an earlier time point, EGR3 remains to be further examined.
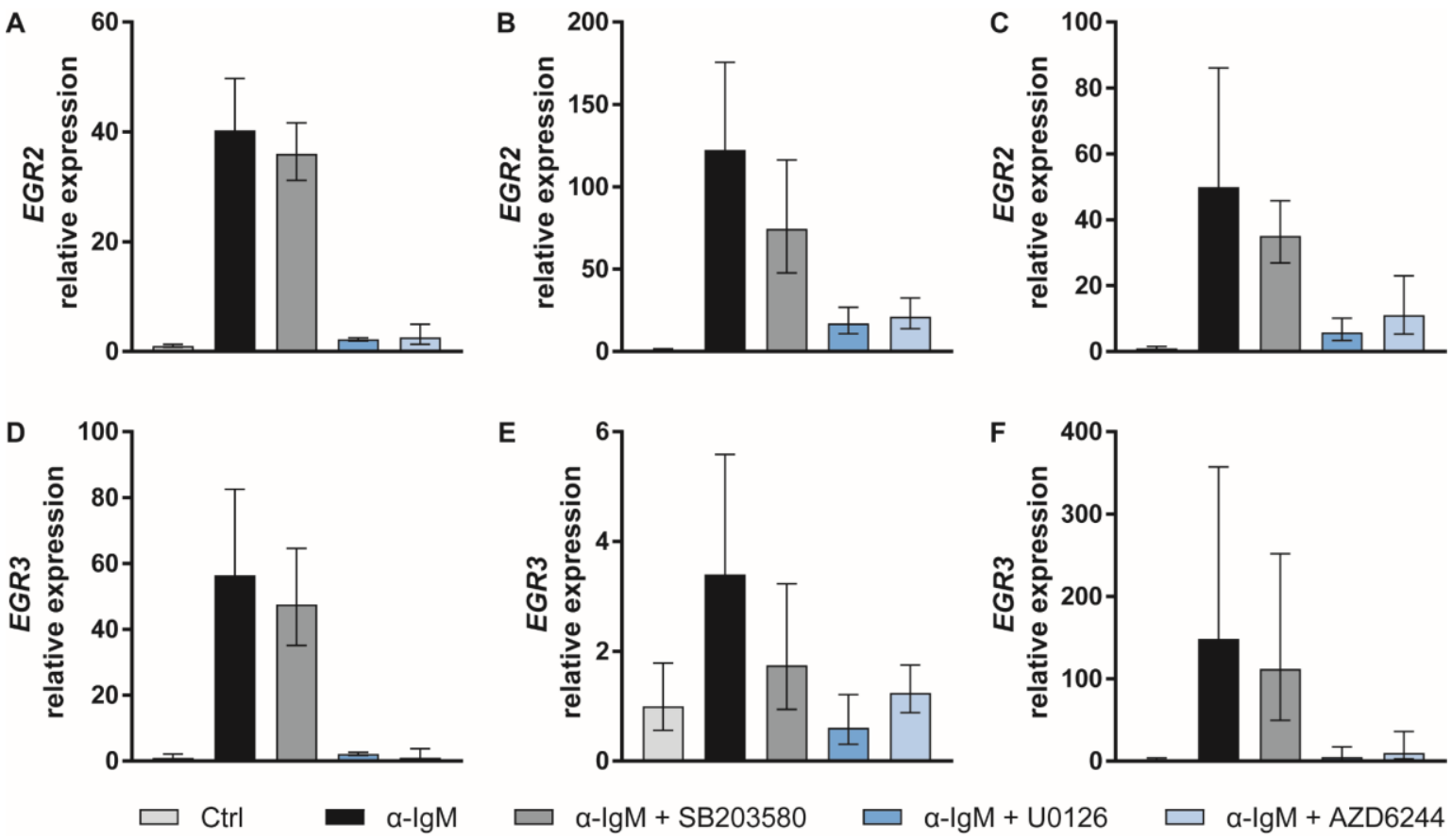

Figure 12: Impact of ERK1/2 and p38 MAPK on early target gene expression

Gene expression of early target genes was analyzed after 3 hours inhibitor treatment following 1 hour BCR activation. Data were normalized to GAPDH (housekeeper) and the untreated control. The mean +/- SD is shown for EGR2 (A - C) and EGR3 (D - F) for the BL cell lines BL-2 (A, D), $\mathrm{BL}-41(\mathrm{~B}, \mathrm{E})$ or CA-46 (C, F) $(\mathrm{n}=3)$.

\subsection{5 p38 MAPK attenuates ERK1/2-regulated gene expression of the kinase MKK6}

To further pursue the analysis of ERK1/2 targets affected by p38 MAPK, we used and re-evaluated the previous generated microarray analyses. In this project, gene expression changes were examined after pathway perturbations during the BCR signaling in BL-2 cells to achieve a network structure indirectly from downstream effects (Hand, 2013). Therefore, we could compare the gene expression changes of MEK1/2 (U0126) and p38 MAPK (SB203580) inhibition during an activated BCR. As p38 MAPK increased the ERK1/2 activity, we examined the expression data for oppositely regulated genes. This comparison and evaluation of the microarray data was performed by Maren Sitte (Statistical Bioinformatics Department Medical Statistics, University Medical Centre Goettingen). The following genes were found to be contrarily regulated after MEK1/2 and 
p38 MAPK inhibition: zinc finger protein 800 (ZNF800), pyruvate dehydrogenase phosphatase 1 (PDP1), special AT-rich sequence-binding protein 1 (SATB1), zinc finger protein 36 C3H1 type-like 1 (ZFP36L1), EGR3, phospholipase D6 (PLD6), myotubularinrelated protein 4 (MTMR4), NACHT, LRR and PYD domains-containing protein 11 (NLRP11), neurogenic locus notch homolog protein 2 (NOTCH2), prostaglandinendoperoxide synthase 1 (PTGS1), CD40 and MAP2K6.

For the validation of these 12 target genes, BL-2 cells were treated in the same way as performed for the microarray analyses and then analyzed with qRT-PCR. Namely, BL-2 cells were pretreated with MEK1/2 or p38 MAPK inhibitors for 3 hours and then the BCR was activated with $\alpha$-IgM for additional 3 hours. The CA-46 cell line was also included in the analyses. The found targets MTMR4, NOTCH2, CD40 were not ERK1/2 or p38 MAPK regulated (data not shown). SATB1 was an ERK target gene but the expression was not altered by p38 MAPK inhibition (data not shown). The expression of EGR3 was already displayed in the previous chapter 3.2.4 while the examination of the other candidates is shown in Figure 13. The microarray data proposed a downregulation of PLD6 by ERK1/2 whereas all other target gene expressions were suggested to be upregulated. A decreased expression of PLD6 was measured after $\alpha$-lgM stimulation in both cell lines (Figure 13 A,D). In addition, p38 MAPK inhibition (SB203580) induced no changes and MEK1/2 inhibition (AZD6244, U0126) was not able to rescue the gene expression to the level of the untreated control.

The expression of the other targets PDP1, ZFP36L1, PTGS1 and MAP2K6 were clearly increased by $\alpha$-IgM stimulation in both cell lines (Figure $13 \mathrm{~B}-\mathrm{C}, \mathrm{E}-\mathrm{F}, \mathrm{G}-\mathrm{H}, \mathrm{J}-\mathrm{K}$ ). Besides, the expressions were induced to a higher extent in BL-2 cells compared to CA-46 cells. The inhibition of MEK1/2 (AZD6244, U0126) reduced the gene expression of PDP1, ZFP36L1, PTGS1 and MAP2K6 to the unstimulated control level thus revealing the dependence on ERK1/2. However, the inhibition of p38 MAPK (SB203580) caused no differences compared to the antigen-stimulated control except for MAP2K6 in CA-46. The expression of MAP2K6 was almost doubled after p38 MAPK inhibition compared with the antigen-stimulated control (Figure $13 \mathrm{~K}$ ). As TNFa can lead to MKK6 (MAP2K6) activation and the expression is known to be ERK1/2 as well as p38 MAPK regulated (Sabio and Davis, 2014), the TNFa gene expression was further investigated (Figure 13 I,L). Interestingly, $T N F \alpha$ was strongly upregulated by BCR and especially ERK1/2 activation (AZD6244, U0126). An influence on the expression of TNFa by p38 MAPK inhibition (SB203580) was not detected. 
To sum up, the target gene expressions of PDP1, ZFP36L1, PTGS1, MAP2K6 and TNFa depended on ERK1/2 activity. However, the increased ERK1/2 activity after p38 MAPK inhibition was only reflected by an increased expression of MAP2K6 in CA-46 cells.
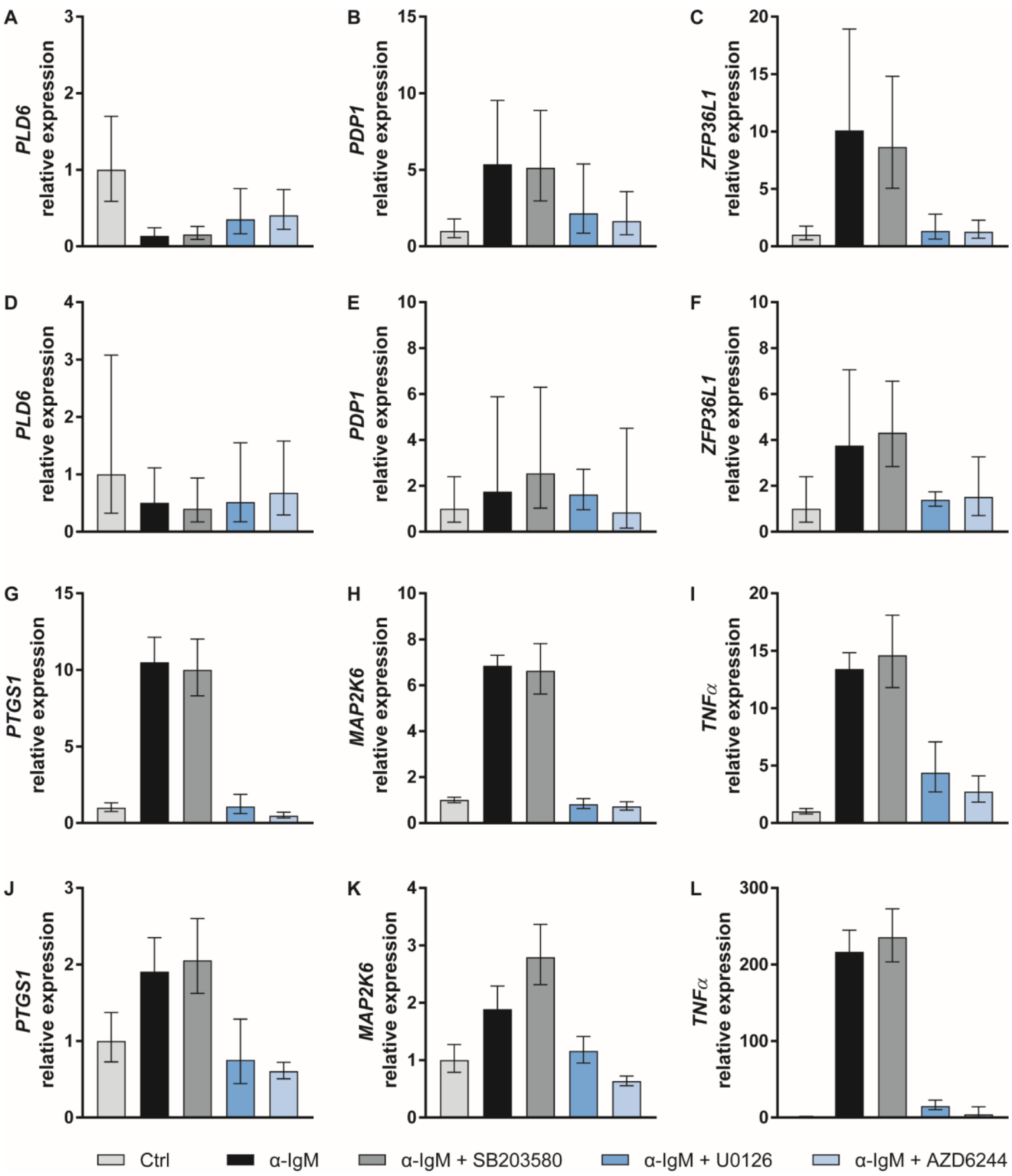

Figure 13: p38 MAPK influence on ERK1/2 target gene expression

Expression of ERK1/2 target genes was measured after 3 hours inhibitor treatment and additional 3 hours BCR activation. Data were normalized to GAPDH (housekeeper) and the untreated control. The mean +/- SD is shown for PLD6 (A, D), PDP1 (B, E), ZFP36L1 (C, F), PTGS1 (G, J), MAP2K6 $(\mathbf{H}, \mathbf{K})$ and $T N F \alpha(\mathbf{I}, \mathbf{L})$ for the BL cell lines BL-2 $(\mathbf{A}-\mathbf{C}, \mathbf{G}-\mathbf{I})$ or CA-46 $(\mathbf{D}-\mathbf{F}, \mathbf{J}-\mathbf{L})(\mathrm{n}=3)$. 
Not to overlook gene expression differences in $\mathrm{BL}-2$ at an earlier time point, the expression of MAP2K6 (MKK6) and the MKK6 activator TNFa was examined after 1 hour of BCR activation (Figure 14). Due to the observed differences between BL-2 and CA-46 cells, the lymphoma cell line BL-41 was included in the analysis. In all three cell lines the $T N F \alpha$ expression was induced by $\alpha$-IgM stimulation in an ERK-dependent manner (AZD6244, U0126) (Figure 14 A-C). The inhibition of p38 MAPK (SB203580) showed no notable impacts on TNF $\alpha$ expression compared to the antigen-stimulated control in BL-2 and CA-46 cells (Figure 14 A,C), while in BL-41 cells p38 MAPK inhibition reduced the expression similar to MEK1/2 inhibition (Figure $14 \mathrm{~B}$ ). The expression analysis of MAP2K6 revealed that BCR activation by $\alpha$-IgM caused a clear induction in BL-2 cells (Figure 14 D) and a slight increase in BL-41 cells (Figure $14 \mathrm{E}$ ). While in BL-2 cells the ERK1/2 dependence (AZD6244, U0126) was obvious, it was only supposed in BL-41 cells. On the contrary, in CA-46 no increased MAP2K6 expression was observed and therefore no ERK1/2-dependence is concluded (Figure $14 \mathrm{~F}$ ). Nevertheless, the inhibition of p38 MAPK (SB203580) resulted in a stronger expression of MAP2K6 in all three cell lines (Figure $14 \mathrm{C}-\mathrm{F})$.
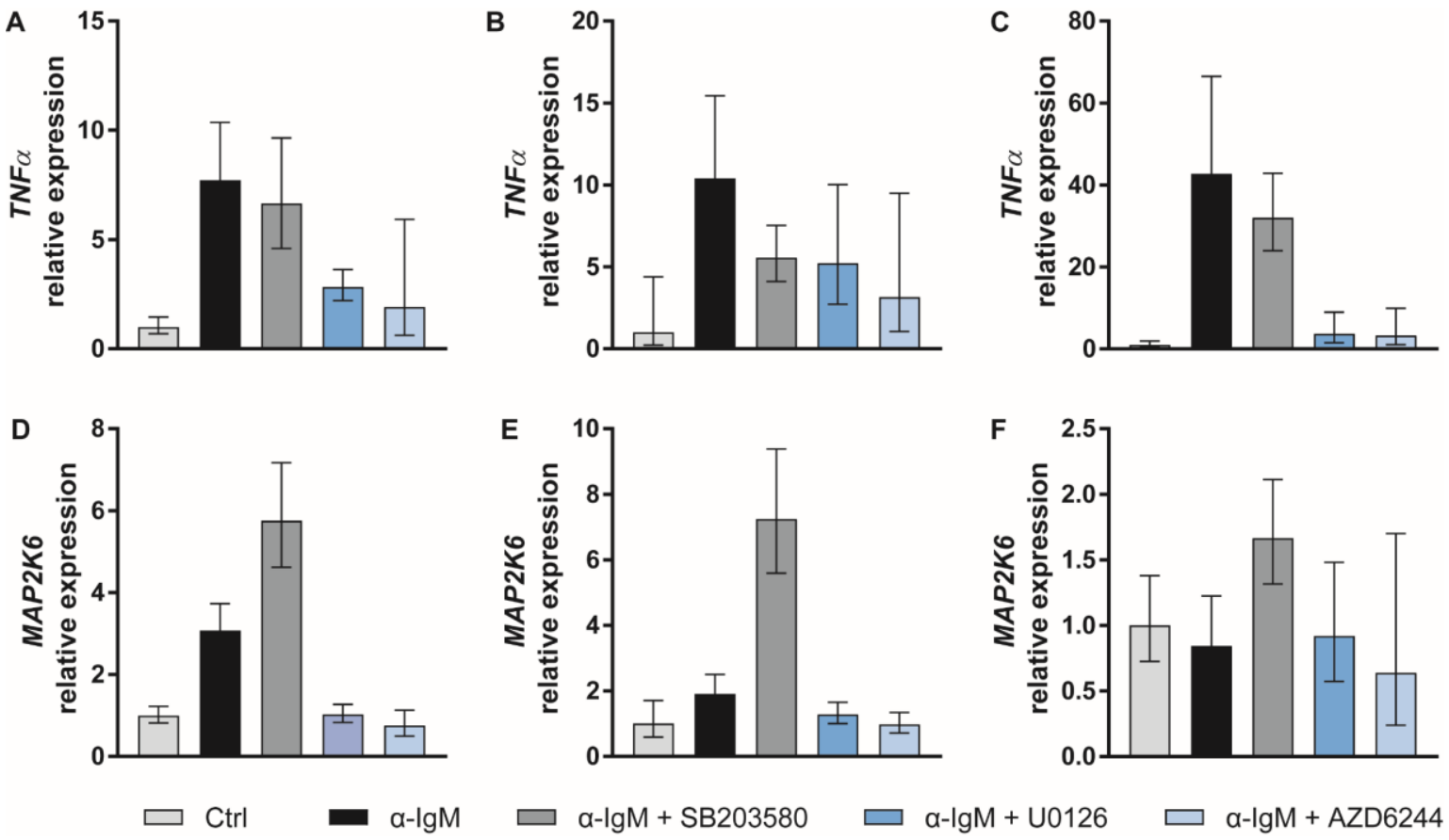

Figure 14: p38 MAPK influence on ERK1/2 target gene expression

Expression of ERK $1 / 2$ target genes was investigated after 3 hours inhibitor treatment and additional 1 hour BCR activation. Data were normalized to GAPDH (housekeeper) and the untreated control. The mean +/- SD is shown for TNFa $(\mathbf{A}-\mathbf{C})$ and MAP2K6 (D - F) for the BL cell lines BL-2 (A, D), BL-41 (B, E) or CA-46 (C, F) ( $n=3)$.

To sum up, p38 MAPK not only attenuated the MEK-ERK pathway, the target gene expression of MAP2K6 was also limited by p38 MAPK after 1 hour of BCR activation. 
Interestingly, MAP2K6 transcribes for the kinase MKK6 which can be activated by TNFa and which is known as upstream activator of p38 MAPK (Sabio and Davis, 2014). Due to the ERK-dependent upregulation of TNFa and MKK6, we suggested that the negative feedback of p38 MAPK on the MEK-ERK pathway can be further promoted by high ERK1/2 activities but this remains to be elucidated.

\subsection{DLBCLs with chronic active BCR contain divers feedback loops}

Unstimulated BLs showed a positive feedback loop of the PI3K pathway and only after BCR activation with antigens two negative feedbacks on the MEK-ERK pathway were revealed. Therefore, the question was whether $B$ cell lymphomas with a chronic active $B C R$ involve the same pathway interplays. In ABC DLBCLs mutations of CD79b, MyD88 or CARD11 mimic an active BCR with subsequent activation of NF-KB (Davis et al., 2010; Ngo et al., 2011). As different mutations in ABC DLBCLs can cause similar pathway activations, the cell lines HBL-1 and OCI-LY3 were chosen. HBL-1 cells harbor mutations in MyD88 and CD79b, while the OCI-LY3 cells are characterized by a receptorindependent activation of NF-KB due to mutations in MyD88 and CARD11 (Paul et al., 2017).

\subsubsection{Positive and negative feedbacks influence chronic active BCR signaling}

In order to compare the feedbacks with BLs, pathway phosphorylations during a chronic active BCR signaling were examined like before using a smaller set of inhibitors than for the BL-2 analysis. The quantitative values of this analysis are presented in the supplement (Figure A-5). In HBL-1 cells we observed that the inhibition of BTK, PI3K, mTOR and JNK reduced the phosphorylation of all measured protein phosphorylations (Figure $15 \mathrm{~A}$ ). More precisely, the activation of SYK, ZAP70, BTK, GSK3 $\beta$ and the PI3K-AKT, MEK-ERK, p38 MAPK, JNK and NF-KB pathways were decreased. On the contrary, the inhibition of AKT increased the phosphorylation of SYK, ZAP70, BTK, GSK3 $\beta$, MEK1, ERK1/2, JNK and c-Jun while the amount of phosphorylated RPS6 was reduced. As a signal inhibition at PI3K or mTOR led to a decreased phosphorylation of downstream and upstream kinases, we suggested that a positive feedback loop from mTOR to the BCR enhanced the signal as observed in BL cells. However, the JNK inhibitor had similar effects like a PI3K inhibitor and the inhibition of AKT revealed an opposite effect although no phosphorylation changes of the downstream target RPS6 were measured. 
Besides, the inhibition of MEK1/2 diminished clearly the ERK1/2 and p90RSK phosphorylation and heightened the MEK1 phosphorylation. This indicated the negative feedback loop from ERK1/2 on its own pathway. The p38 MAPK inhibition heightened strongly the MEK1 and ERK1/2 phosphorylation suggesting that p38 MAPK had even a negative impact on the MEK-ERK pathway. The simultaneous inhibition of p38 MAPK and MEK $1 / 2$ revealed that the influence on the pathway occurred mainly upstream of ERK $1 / 2$ as ERK1/2 phosphorylation was reduced around $85 \%$ compared to p38 MAPK inhibition alone. Finally, the NF-KB inhibition just slightly reduced the phosphorylation of SYK, ZAP70, BTK and of the PI3K-AKT, p38 MAPK and NF-KB pathway, whereas an increased phosphorylation of MEK1 was detected. However, NF-KB seemed not to be a further regulator of the MEK-ERK pathway because no effects on ERK1/2 were measured.

A

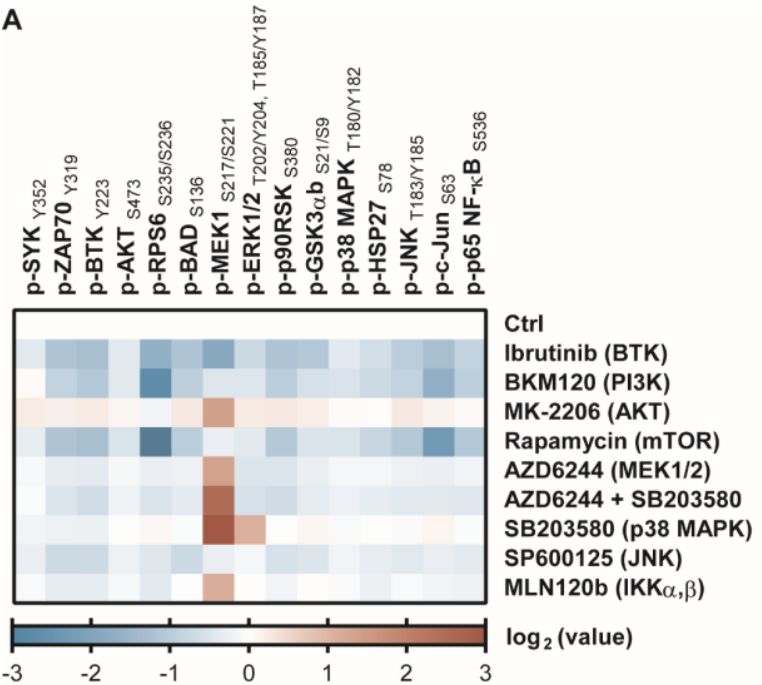

B

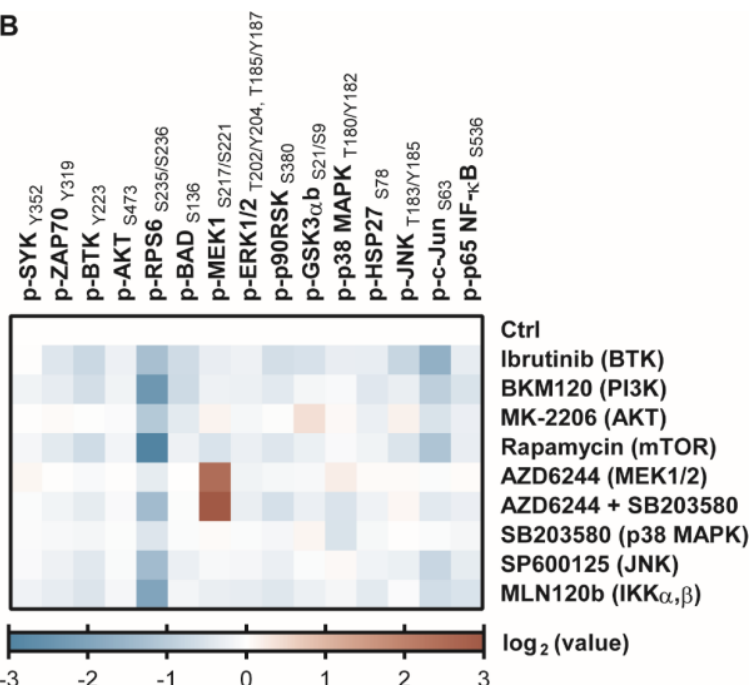

Figure 15: Signaling changes by pathway perturbations during the chronic active BCR signaling.

The signal transduction were disrupted by inhibitors of BTK, PI3K, AKT, mTOR, MEK1/2, p38 MAPK, JNK and IKKa, $\beta$ for 3 hours. Specific phosphorylations of SYK, ZAP70, BTK, AKT, RPS6, BAD, MEK1, ERK1/2, p90RSK, GSK3ab, p38 MAPK, HSP27, JNK, c-JUN and p65 NF-KB were measured with the multiplex immunoassay. The $\log _{2}$ values for the $A B C$ DLBCL lymphoma cell line HBL-1 (A) or OCI-LY3 (B) are shown normalized to the control $(n=3)$.

The treatment of OCI-LY3 cells caused mostly the same effects though the overall phosphorylation changes were smaller (Figure $15 \mathrm{~B}$ ). Therefore, only the differences to HBL-1 cells are described. The inhibition of AKT caused a diminished phosphorylation of RPS6 and similar effects like PI3K and mTOR inhibition. Only the phosphorylation of GSK $3 \beta$ was raised and therefore controversial to HBL- 1 cells. The inhibition of MEK1/2 had no influence on p38 MAPK in HBL-1 cells, whereas in OCI-LY3 cells a slight increased phosphorylation was observed. Furthermore, the NF-KB inhibition did not lead 
to higher MEK1 phosphorylations as seen in HBL-1 cells. However, the most obvious difference was that inhibition of p38 MAPK had no impact on the MEK-ERK pathway.

In conclusion, we suggested that the positive feedback of the PI3K signaling and the negative feedback from ERK1/2 on the MEK-ERK pathway is conserved in $B$ cell lymphomas. Nevertheless, we assumed that due to genetic variations the regulation of the MEK-ERK pathway by p38 MAPK differs between the ABC DLBCL cell lines. This indicated that the genetic background can influence possible interactions and feedbacks and thereby the signal distribution.

The obvious assumption for the missing influence of p38 MAPK on the MEK-ERK pathway could be that the p38 MAPK inhibitor was not functioning. But due to the slight reduction of phosphorylated p38 MAPK after the inhibitor treatment in several analyses, the three biological replicates and the simultaneously performed HBL-1 analyses, this possibility was excluded. Furthermore, the missing effect could be explained by an inactive MEK-ERK signaling. Comparing BL-2, HBL-1 and OCI-LY3 cells, it was shown that OCI-LY3 had phosphorylated ERK1/2 and furthermore high levels of phosphorylated p38 MAPK (Figure 16 A). Therefore, the cellular state of OCI-LY3 cells were suggested to be responsible for the missing negative feedback on the MEK-ERK pathway. One explanation can be that different mutations like the mutation of CARD11 entail an altered expression of some proteins. However, this would not be an explanation for the missing p38 MAPK effect if p38 MAPK directly interacted with RAF-1. But due to the not known interaction between p38 MAPK and RAF-1, we suggested that a p38 MAPK regulated phosphatase would rather explain the cell line differences as it could reduce any phosphosites in the MEK-ERK pathway.

In order to investigate kinases or phosphatases differently expressed in HBL-1 and OCl-LY3 cells, the gene expression of two previous RNA-sequencing projects were analyzed. Therefore, the estimated RNA sequencing reads of $\mathrm{BL}-2, \mathrm{HBL}-1$ and OCI-LY3 cells were compared to find a missing kinase or phosphatase expression in OCI-LY3 cells (Figure $16 \mathrm{~B}$ ). An overview of phosphatases and kinases with more than $50 \%$ differential expression between HBL-1 and OCI-LY3 cells is displayed. The extract of the gene list revealed that some phosphatases were highly expressed by HBL-1 and BL-2 cells and nearly absent in OCI-LY3 cells. For instance, the lymphocyte cell-specific protein tyrosine kinase (LCK), paladin (PALD1), tyrosine protein phosphatase non-receptor type 22 (PTPN22) and PTPN7 were barely expressed in OCI-LY3 cells which could explain the missing p38 MAPK influence on the MEK-ERK pathway. However, this hypothesis remained to be proven. 
A

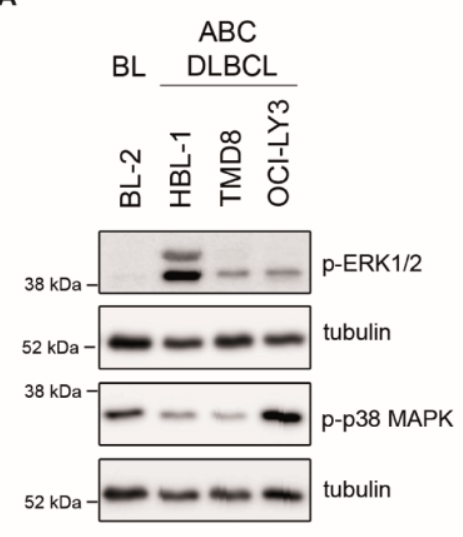

B

\begin{tabular}{|c|c|c|c|}
\hline & OCI-LY3 & BL-2 & \\
\hline 253.00 & 2.50 & 4062.67 & LCK \\
\hline 1164.50 & 470.50 & 2219.45 & PALD1 \\
\hline 1530.50 & 803.50 & 2986.37 & RNGTT \\
\hline 1385.00 & 302.00 & 982.87 & PTPN22 \\
\hline 3069.50 & 447.00 & 1449.95 & PTPN7 \\
\hline 1720.50 & 819.00 & 2131.22 & PTEN \\
\hline 485.00 & 309.00 & 668.97 & CAMK2C \\
\hline 1410.50 & 636.50 & 1230.07 & SSH2 \\
\hline 802.00 & 218.50 & 388.28 & DNAJC6 \\
\hline 433.00 & 12.50 & 20.72 & MTMR7 \\
\hline 718.00 & 263.50 & 411.01 & PTPN12 \\
\hline 5558.50 & 3380.50 & 5215.48 & PTPRC \\
\hline 145.50 & 724.50 & 332.07 & CPPED1 \\
\hline 2319.50 & 4923.50 & 1765.17 & PPP5C \\
\hline 2331.00 & 5717.50 & 2026.51 & PGAM5 \\
\hline 444.50 & 1595.00 & 502.19 & PTPRG \\
\hline 6.50 & 145.50 & 34.80 & PTPRF \\
\hline 135.00 & 308.00 & 31.84 & DUSP1 \\
\hline 406.00 & 1195.50 & 120.71 & DUSP10 \\
\hline 78.00 & 1331.00 & 130.53 & PPM1E \\
\hline 1108.00 & 2871.00 & 131.76 & PTPRE \\
\hline 4557.50 & 33575.50 & 1361.19 & PTPN1 \\
\hline 24.50 & 223.00 & 4.90 & CDC14B \\
\hline 7.00 & 203.00 & 0.94 & DUSP4 \\
\hline 36.00 & 840.00 & 1.32 & DUSP15 \\
\hline 14.50 & 233.00 & 0.31 & PPM1H \\
\hline 186.50 & 393.50 & 0 & PTPDC1 \\
\hline
\end{tabular}

010002000300040005000

Figure 16: Comparison of BL-2, HBL-1 and OCI-LY3 cells on protein and RNA level

To explain the cell line differences of the multiplex immunoassay BL-2, HBL-1 and OCI-LY3 cells were compared on protein and RNA level. (A) Representative immunoblot analysis of the different, untreated lymphoma cell lines. Tubulin served as loading control $(n=3)$. (B) An extract of phosphatases and kinases of the RNA sequencing analyses with mean of estimated counts are displayed for HBL-1, OCI-LY3 and BL-2 cells $(n=3)$.

\subsubsection{Negative feedbacks of the chronic active BCR signaling have no impacts on proliferation}

While the inhibition of BTK, PI3K, JNK and NF-KB is well studied in DLBCLs (Gururajan et al., 2005; Kloo et al., 2011; Young and Staudt, 2013), less is known about the impact of MEK1/2 or p38 MAPK inhibition on proliferation. Only one paper indicated that inhibition of MEK1/2 with the AZD6244 inhibitor induced apoptosis in OCI-LY3 cells after 48 hours (Bhalla et al., 2011). Therefore, the inhibition of MEK1/2 and p38 MAPK was analyzed on cell viability over 72 hours (Figure 17). No influences on cell viability of HBL-1 cells were detectable (Figure $17 \mathrm{~A}$ ) and OCI-LY3 cells showed only a small decrease of cell viability about 15 - $20 \%$ after MEK1/2 and p38 MAPK inhibition (Figure 17 B). Consequently, the inhibition of ERK1/2 or p38 MAPK did not reduce considerably the cell viability.

This indicated that the negative feedbacks on the MEK-ERK pathway are not involved in the regulation of cell proliferation and therefore the specific role of MAPKs in lymphoma needs to be further elucidated. 
A

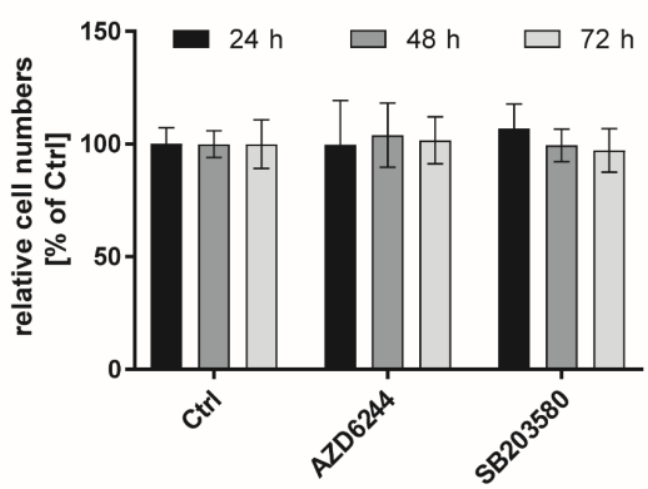

B

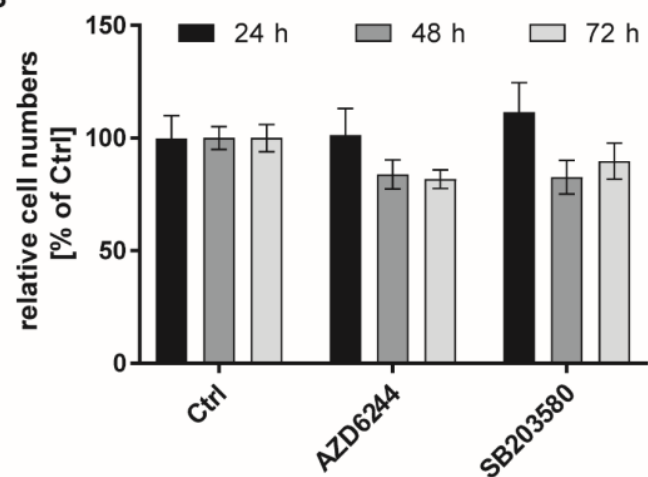

Figure 17: Cell viability of HBL-1 and OCI-LY3 cells after MEK1/2 or p38 MAPK inhibition Cell viability assay was performed after 24,48 and 72 hours with the fluorescence dye Calcein AM. The amount of viable cells was normalized to control and the mean $+/-\mathrm{SD}$ is displayed for HBL-1 (A) and OCI-LY3 (B) cells $(n=3)$.

\subsection{IL10R and TLR9 activation modify cell response and behavior}

Apart from the several signaling pathways of the chronic active $B C R, A B C$ DLBCLs contain an autocrine JAK-STAT activation. This is mediated by a BCR-dependent NF-KB signaling which causes an increased expression of ILS and thereby subsequently stimulates IL receptors and JAKs (Gupta et al., 2012; Jost and Ruland, 2007). Our group has previously described that only the combined activation of NF-KB and STAT3 induces proliferation in a synergistic manner (Feist et al., 2017; Feist et al., 2018). To further examine mediators of the proliferative boost and the directly altered cellular processes after receptor activation, a phosphoproteomics analysis was performed. As ABC DLBCLs differ in their behavior due to cell line specific mutations, the analyses were done with the model cell line P493-6. This allows a direct comparison of cells with active or inactive NF-KB and STAT3 without variations resulting from mutations.

\subsubsection{IL10R and TLR9 activation change 239 protein phosphorylations}

The examination of global protein phosphorylations was done after TLR9 and IL10R activation with mass spectrometry. For an accurate comparison of two conditions, the cells were labelled with stable isotopes (SILAC). In Figure $18 \mathrm{~A}$ the workflow is presented, the heavy labeled P493-6 cells (Myc ${ }^{\text {high}}$ ) were left untreated while the light labeled cells were supplemented with doxycycline to reduce the amount of c-MYC ( $\mathrm{Myc}^{\mathrm{low}}$ ). Afterwards Myc ${ }^{\text {low }}$ cells were stimulated with IL $10+C p G$ to activate TLR9 and IL10R for 30 minutes or left untreated as control (Ctrl). The Mychigh cells served as a reference control for normalization and thus were also activated by IL $10+\mathrm{CpG}$ stimulation for 30 minutes to 
obtain sufficient protein phosphorylations. Heavy and light labeled conditions were mixed in equal parts, the protein phosphorylation was determined and the phosphorylation changes of control or IL10+CpG stimulated Myc ${ }^{\text {low }}$ cells in relation to Myc ${ }^{\text {high }}$ cells were calculated.

A

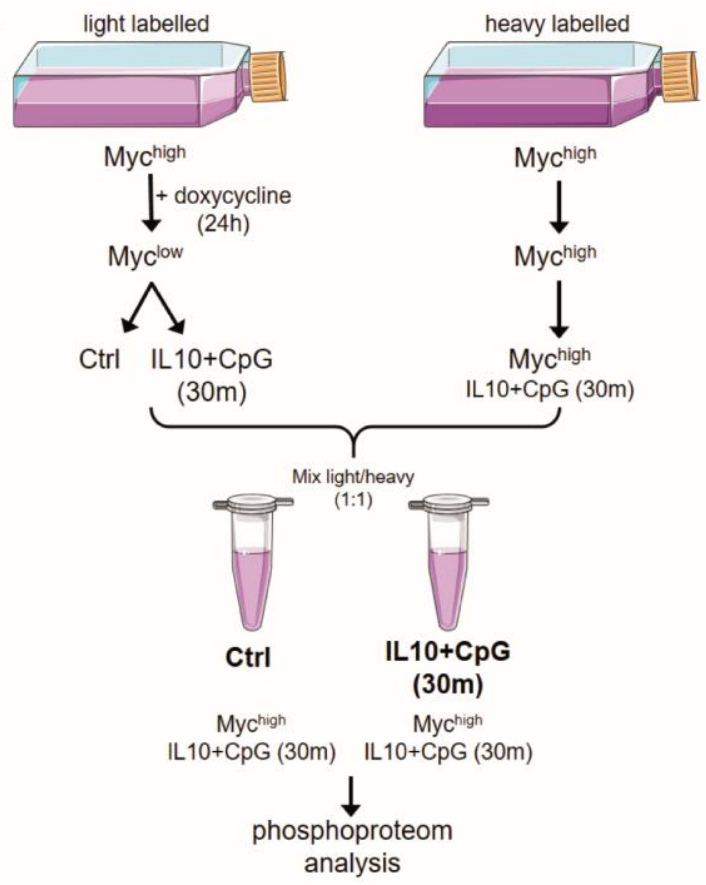

B

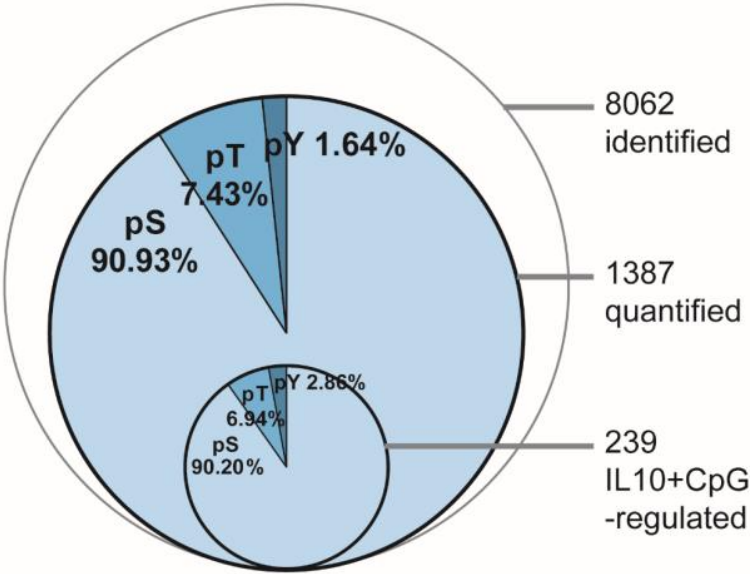

Figure 18: Workflow and global phosphosites identification of mass spectrometry analysis To reveal protein phosphorylations Ctrl and IL10+CpG stimulated Myclow cells were analyzed in relation to Mychigh cells with mass spectrometry $(n=3)$. (A) Schematic workflow for labeling, stimulating and harvesting of $\mathrm{Myc}^{\mathrm{low}}$ and Mychigh cells. (B) Proportion of phosphorylated residues of quantified and IL10+CpG-regulated proteins.

Out of 8062 detected phosphosites, 1387 were quantified in at least two out of three replicates and with a p-value less than 0.05 (Figure $18 \mathrm{~B}$ ). The stimulation of TLR9 and IL10R led to significant change of 239 phosphosites with a phosphorylation differences between Ctrl and IL10+CpG stimulated Myc ${ }^{\text {low }}$ cells of more than $50 \%$. Thereby, the proportion of threonine, tyrosine and serine phosphorylated residues was not altered between quantified and IL10+CpG-regulated phosphosites. This proportion accurately reflects the relative abundance of serine, tyrosine and threonine phosphorylations after receptor activation (Olsen et al., 2006).

In order to compare the results of the biological replicates, the phosphorylation ratios for the Ctrl (Figure $19 \mathrm{~A}$ ) and IL10+CpG stimulated (Figure $19 \mathrm{~B}$ ) Myc ${ }^{\text {low }}$ cells in relation to Myc $^{\text {high }}$ cells were plotted for each biological replicate against the other replicates. The 
Pearson's correlation coefficient was similar for each comparison of the biological replicates and ranged from 0.78 to 0.88 indicating an acceptable reproducibility.

A

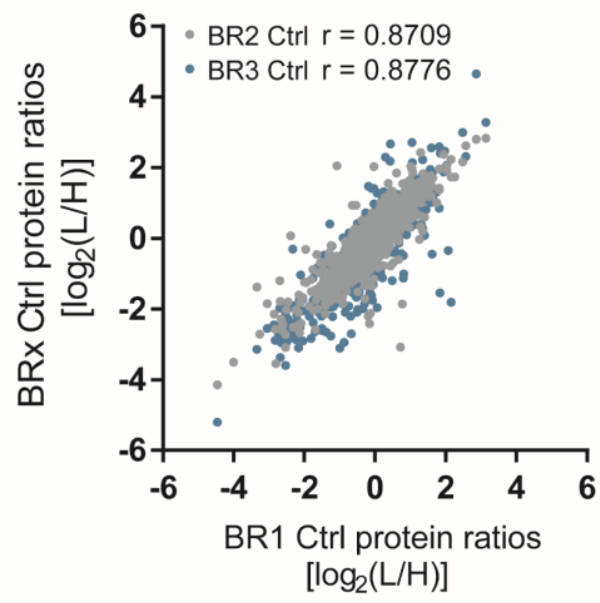

C

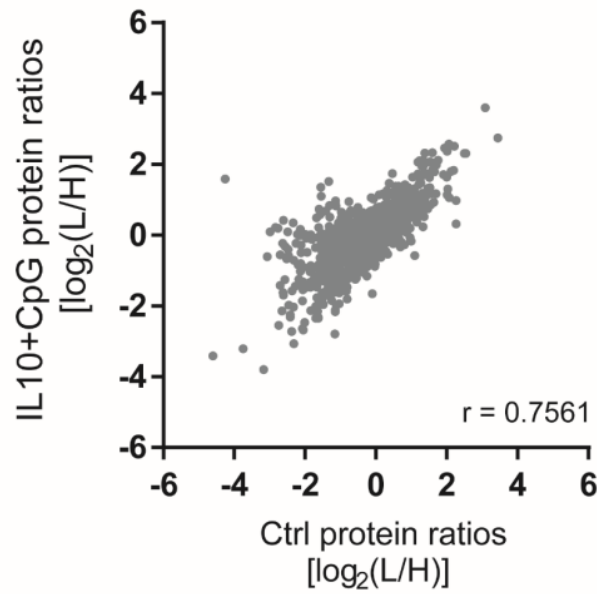

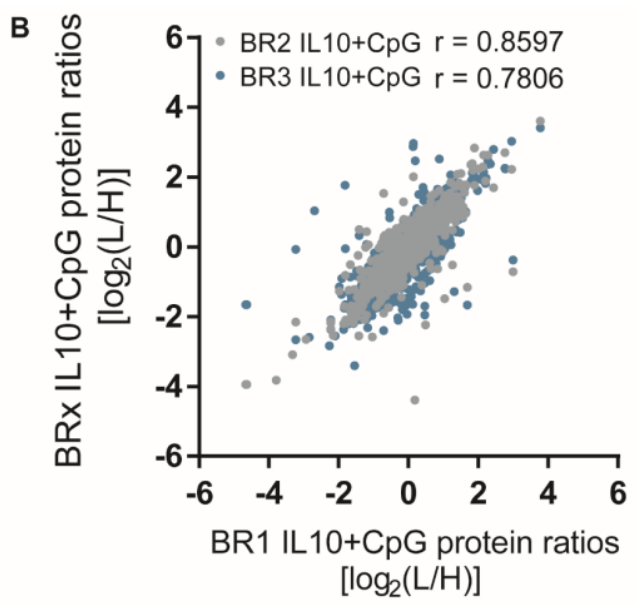

D

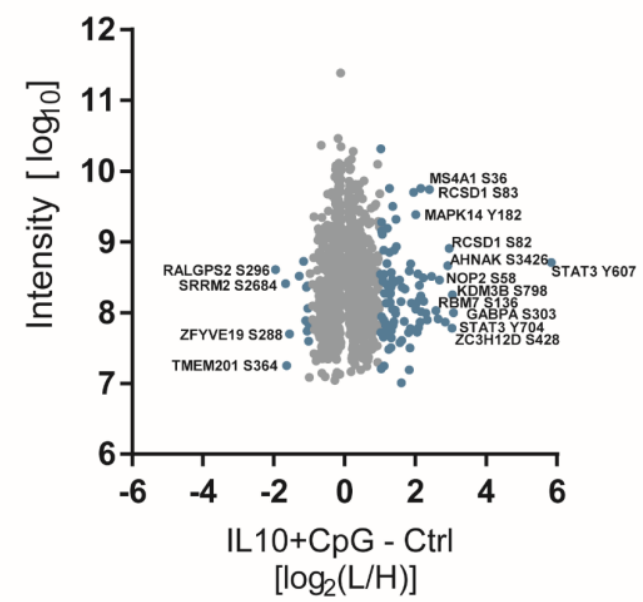

Figure 19: Global phosphoproteomics results of Ctrl and IL10+CpG stimulated Myc ${ }^{\text {low }}$ cells Scatter Plots with Pearson's correlation coefficients were demonstrating the reproducibility of biological replicates for Ctrl (A) or IL10+CpG stimulated (B) Myclow cells. (C) Scatter Plot with Pearson correlation coefficients compared the values of quantified phosphosites of Ctrl and IL10+CpG stimulated Myclow cells. (D) Scatter plot showed the measured intensities for the $\log _{2}$ ratios of IL10+CpG stimulated Myclow cells in relation to the Ctrl. Log $_{2}$ ratios with difference above 1.0 are indicated in blue. All quantified phosphosites are listed in the supplement.

To present the phosphosite changes after IL10+CpG stimulation, the phosphorylation ratios of the IL10+CpG stimulated Myc ${ }^{\text {low }}$ cells were displayed to the ratios of the Ctrl Myc $^{\text {low }}$ cells (Figure $19 \mathrm{C}$ ). As more phosphosite ratios were higher in IL10+CpG stimulated cells compared to Ctrl, we suggested that the stimulation of cells clearly induced a phosphorylation of proteins while only few sites were dephosphorylated. The precision of quantitation increased with the protein abundance, therefore the measured intensities were displayed over the relation of IL $10+C p G$ stimulation to the Ctrl Myc ${ }^{\text {low }}$ cells (Figure $19 \mathrm{D}$ ). Protein phosphorylations with a $\log _{2}$ change higher than 1.0 are marked (blue dots). For example, the STAT3 tyrosine phosphorylation known to be induced by 
IL10 was strongly increased after IL10+CpG stimulation as well as some further sites of p38 MAPK (MAPK14), capZ-interacting protein 1 (RCSD1) and nucleolar protein 2 homolog (NOP2). The full list of the 239 changed phosphosites is presented in Table A-1.

\subsubsection{Phosphoproteome reveals influence on cell cycle, metabolism and migration}

Identifying the connection of regulated proteins is valuable in order to build a network which can help to reveal closely related proteins and altered protein groups. Therefore, the IL10+CpG-influenced phosphosites were used for a network generation with STRING (Figure 20).

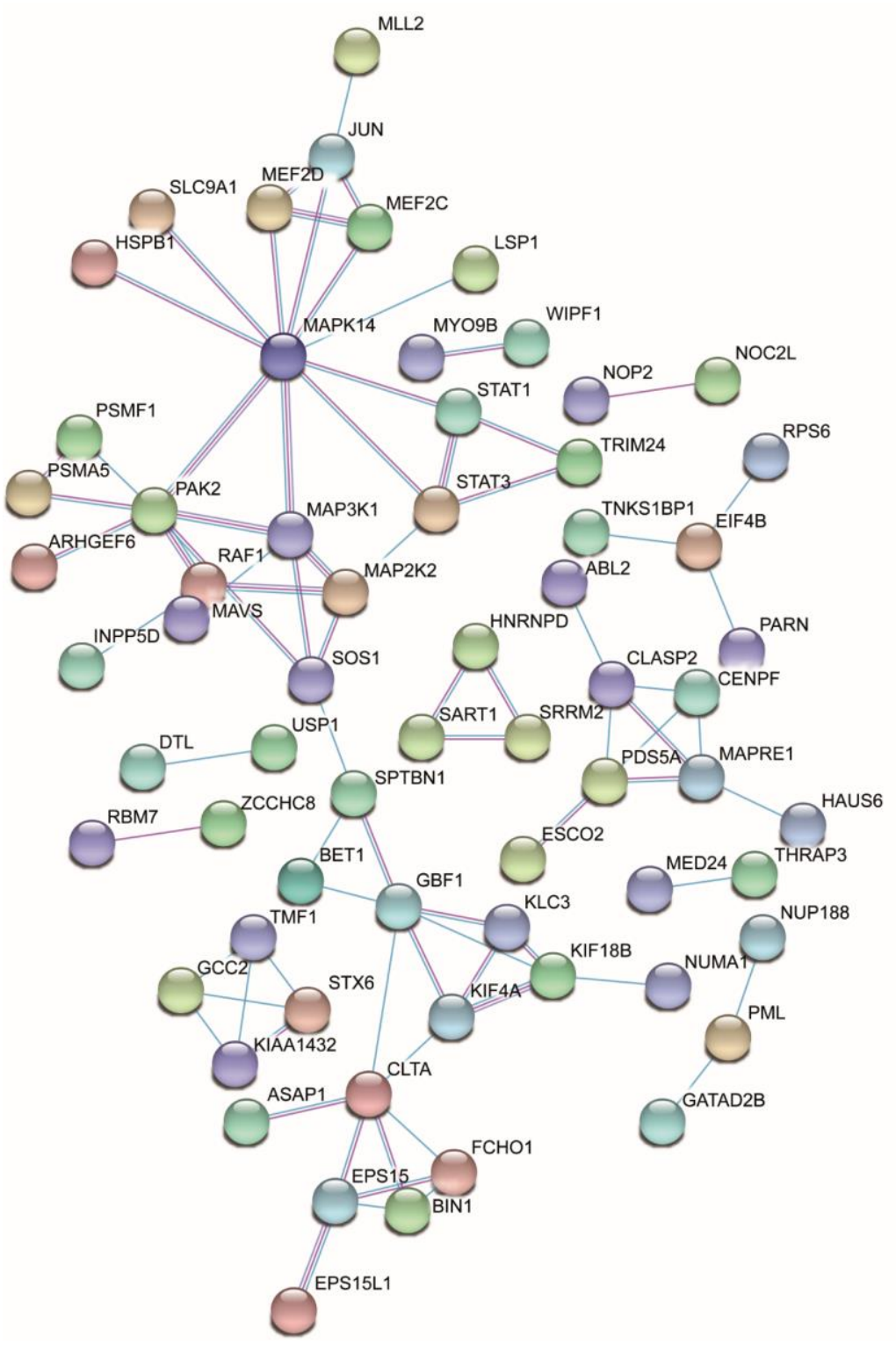

Figure 20: Network of affected phosphoproteins after IL10+CpG stimulation

Phosphoproteins that were up- or down-regulated by stimulation with IL10+CpG were used for an interaction analysis with STRING. The known interactions from curated databases (blue) and experimentally determined (red) are displayed. 
The network revealed an interaction of proliferation associated proteins like RPS6, Poly(A)-specific ribonuclease (PARN) and EIF4B. Some MAPK-related proteins such as JUN, MAP3K1 and MAP2K2 clustered around p38 MAPK (MAPK14). This pointed out that, beside NF-KB and STAT3 activation, MAPKs were affected by TLR9 and IL10R activation to probably induce proliferation-associated proteins.

Previously, our group showed by RNA sequencing analyses that the impact of IL10+CpG stimulation alters the expression of cell cycle and metabolism-related genes (Feist, 2016). On the basis of phosphorylated proteins we further investigated the biological processes affected by the TLR9 and IL10R activation. Therefore, the 239 phosphosites altered after IL10+CpG stimulation were used for an enrichment of Gene Ontology (GO) terms with the functional annotation clustering tool of DAVID (Table A-2; Figure A-6). As the regulated phosphosites were part of many different biological processes, the list of GO terms was visualized in a tree map by REVIGO (Figure 21). Interestingly, the main altered biological process was also cell cycle. Furthermore, positive regulation of molecular function, cellular localization and protein phosphorylation were influenced by IL10+CpG stimulation. More precisely, DNA or mRNA metabolism, signal transduction, ameboidal-type cell migration as well as cell-cell adhesion belonged to altered processes.

In conclusion, we assumed that MAPK activation and the induction of proliferationassociated proteins may contribute to the proliferative effect after IL10+CpG stimulation. In addition, the protein phosphorylation after 30 minutes of receptor activation pointed to influences on cell cycle, metabolism and movement of cells. 


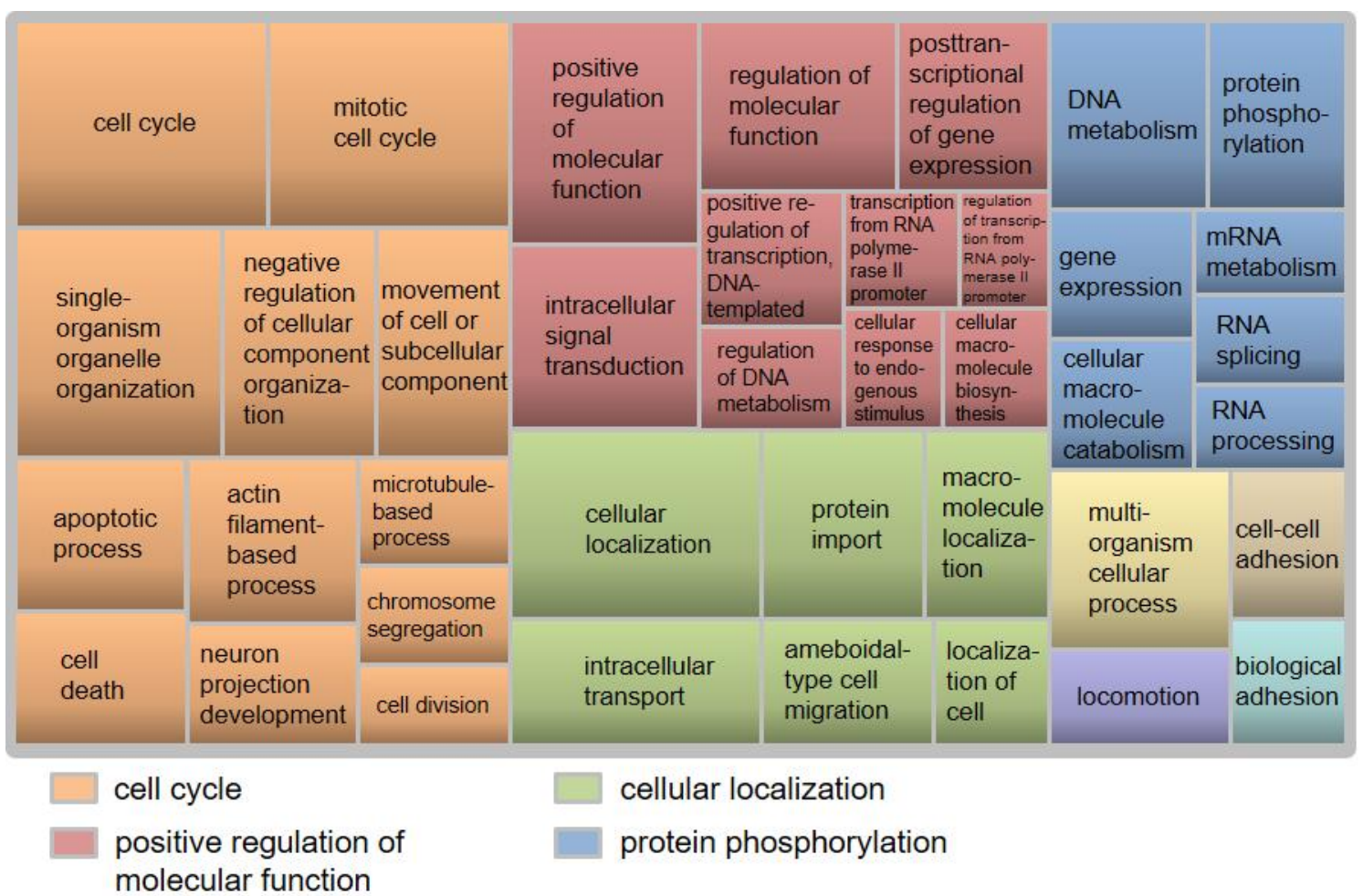

Figure 21: GO annotation of biological process changed by IL10+CpG stimulation

Biological processes that were up- or down-regulated by stimulation with IL10+CpG were identified using the 239 regulated phosphosites and the annotation tool DAVID. REVIGO was used for summarization and visualization of GO terms in a tree map. Clusters of related terms are marked with the same color and the size of boxes displays the $p$-values.

\subsubsection{Phosphoproteome analysis indicates MAP kinase involvement}

To reveal further mediators of the proliferative effect, an overview of the involved signaling components was required. Therefore, the phosphoproteins altered after TLR9 and IL10R activation were analyzed with the KEA2 tool to identify the possible upstream kinases and activated pathways (Figure $22 \mathrm{~A}$ ). Beside the STAT3 activating kinases JAK1 and JAK2, the MAPK activation seemed to be further involved through JNK (MAPK9), MKK6 (MAP2K6) and p21-activated kinase 2 (PAK2). Furthermore, the cell cycle regulator CDK2 was listed. The kinase enrichment led to the assumption that MAPK as well as a direct phosphorylation of CDK2 were involved in the initiation of proliferation. As previous findings of our group uncovered that the combined activation of STAT3 and NF-KB induced the gene expression of CDK4 and thereby enhanced the proliferation of cells, the gene expression changes of these predicted kinases were considered (Figure $22 \mathrm{~B}$ ). Similar to CDK4 and CDK6, the expression of CDK2 was enhanced and to a lower extent the expression of JAK1, JAK2, MAPK9 and PAK2 after IL10+CpG stimulation in Myclow cells. 
We concluded that the stimulation with IL10+CpG induces probably not only CDK4 and CDK6 expression as published before (Feist, 2016), furthermore, some proliferation associated proteins seem to be enhanced in their expression as well as in their direct phosphorylation. The impact of these possible mediators remains to be investigated.

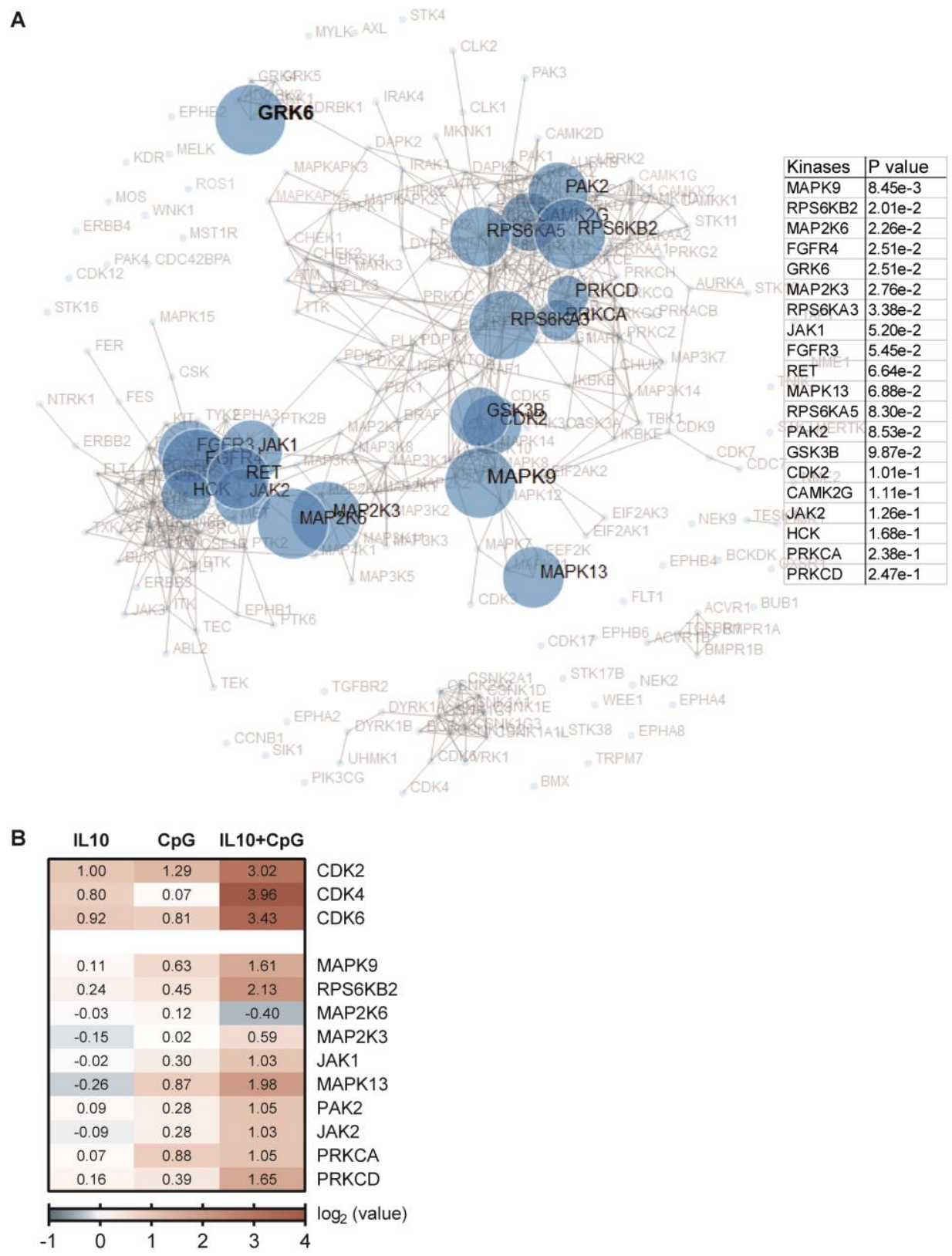

Figure 22: IL10+CpG stimulation caused phosphorylation and expression of CDKs and MAPKs

(A) Network of predicted upstream kinases for the changed 239 phosphosites using the kinase enrichment analysis (KEA) tool. Node size indicated the number of changed phosphosites which were predicted to be phosphorylated by that kinase. (B) RNA expression after 24 hours of proteins relevant in $A$. Displayed are the $\log _{2}$ fold changes after different stimulations normalized to Ctrl Myclow cells $(n=3)$. 


\subsubsection{IL10R and TLR9 activation reduce migration capacity of P493-6 cells}

Beside the known influence on cell cycle and metabolism, the phosphoproteomics analysis pointed to an altered movement of cells. To investigate which phosphoproteins were involved in this process, those out of the 239 changed phosphosites are displayed that were annotated to the GO term "movement of cell or subcellular component" (Figure $23 \mathrm{~A})$. The receptor activations mainly led to an increased phosphorylation of movement associated proteins. Several proteins well-known to play a role in migration were listed like p21-activated kinase 1 (PAK1), STAT3 and SOS1. Therefore, the cell movement was analyzed with the Boyden chamber assay (Figure $23 \mathrm{~B}$ ). The migration potential of stimulated Myc ${ }^{\text {low }}$ cells through $8 \mu \mathrm{m}$ membranes for 6 hours was shown in relation to the migration potential of the unstimulated Ctrl Myc ${ }^{\text {low }}$ cells. While IL10 stimulation alone had no effect on the migration, the TLR9 activation by $\mathrm{CpG}$ reduced the movement potential of Myc ${ }^{\text {low }}$ cells about $30 \%$. Nevertheless, combined receptor activation led to a reduction of $50 \%$ in comparison to Myc ${ }^{\text {low }}$ cells.

In conclusion, many proteins associated with migration were changed by IL10+CpG stimulation. The phosphorylation of these proteins was mainly upregulated. Interestingly, the overall effect of this upregulation after stimulation was a reduced migration of the cells. The effect of the identified phosphorylations and how these proteins interact to regulate migration will need further investigations. 
A
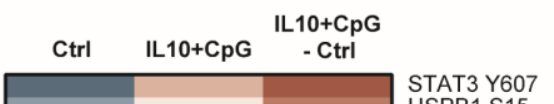
\begin{tabular}{ll}
\hline STAT3 Y704 \\
MYO9B T1352 \\
MPF
\end{tabular} MEF2C S226
MYO9B S1411
PAK1 T114 PAK1 T114
MAPK14 Y182 KIF S100
KIIA S804
DYNC1LI1 S516
MYO9B S1273
MAP1B S1852 MAP1B S1852 MYO9B S1296
CLASP2 S934 HSPB1 S82
ARHGAP4 S340
CLTA S105 \begin{tabular}{|l|l|}
\hline & AMFR S191 \\
MAPRE1 S155 \\
SNAPIN T14 \\
PRKD2 S198
\end{tabular} MEF2C S220 ABL2 S936 KIF18B S94 NUP188 S1709 RAF1 S609 MEF2C S238 SLC9A1 364 SLC9A1 5364 NPP5 5971 MKL1 454 MKL1 S454 KLC2 S610 STK2 4 S4 WIPF1 S33 TP

15225

PML S518

PML 527

PML 527

CD2AP S458

TMEM201 S364

$\log _{2}$ (value)

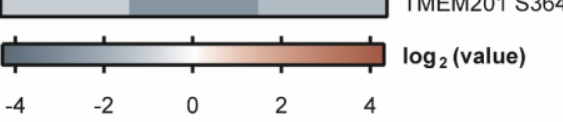

B

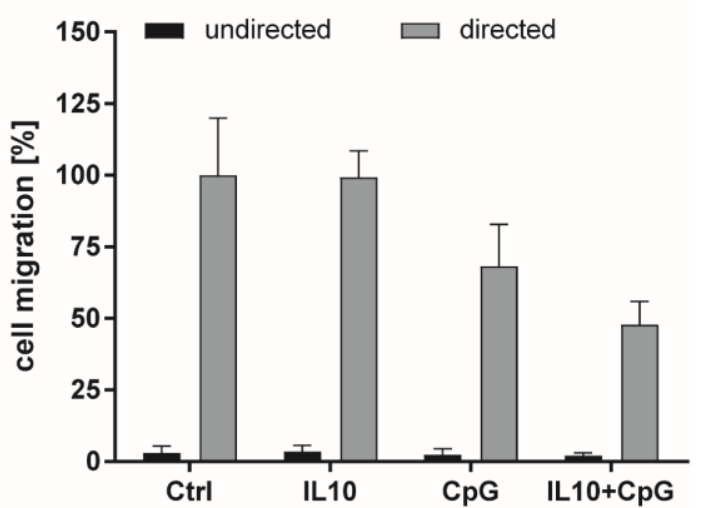

Figure 23: IL10+CpG impact on movement associated proteins and the migration potential Investigation of the influence on movement associated proteins by TLR9 and IL10R activation. (A) Phosphoprotein changes of Ctrl and IL10+CpG stimulated Myclow cells were displayed as well as the difference between the values of Ctrl and IL10+CpG stimulated Myclow cells. (B) Boyden chamber analysis with $8 \mu \mathrm{m}$ pore size membrane. Myclow cells were allowed to migrate for 6 hours towards $10 \%$ FCS (directed) or $0 \%$ FCS (undirected) after IL10, CpG or combined stimulation. Cell counts were normalized to the directed migration of the Ctrl Myc ${ }^{\text {low }}$ cells $(n=3)$. 


\section{Discussion}

Many B cell non-Hodgkin lymphomas depend on the BCR signaling (Young and Staudt, 2013). Targeting BCR-related kinases emerges as a promising therapeutic strategy for several B cell malignancies (Smith, 2015). Nevertheless, the occurrence of unexpected side effects and toxicities is limiting the therapeutic success as feedback mechanisms are often disregarded (Blachly and Baiocchi, 2014). In the literature, mostly the linear signaling cascade from the receptor to the nucleus is displayed, while the interplays and feedback loops of pathways are often neglected (Reth and Brummer, 2004). This is also the case for the BCR signaling as the proximal events after receptor activation are well investigated but an accurate network model is still missing (Corso et al., 2016; Satpathy et al., 2015). Therefore, the downstream interplay of pathways remains to be examined for a further refinement of the signaling network. First approaches were performed by our group with Boolean-Nested Effect Models on the basis of gene expression changes after pathway perturbations (Pirkl et al., 2016). However, pathway interplays and feedbacks hampered the network elucidation and thus an additional examination of pathway activations was required. The aim of this thesis was to uncover pathway interplays and feedbacks during the tonic, activated and chronic active BCR signaling to generate a semi-quantitative network model. In addition, the BCR-induced NF-kB activation with subsequent JAK-STAT activation is so far not examined for further pathway interactions. Thus, the interplay of TLR9 and IL10R signaling was investigated to identify additional mediators contributing to the NF-KB and JAK-STAT-dependent proliferative effect in the model cell line P493-6.

\subsection{Positive feedback loop enhances tonic BCR signaling}

The tonic BCR signaling is essential for the survival of BLs (Schmitz et al., 2012). Aberrant regulation of C-MYC with additional activation of the PI3K pathway is sufficient to cause BL-like lymphomagenesis (Sander et al., 2012; Schmitz et al., 2012). An enhanced $\mathrm{PI} 3 \mathrm{~K}$ pathway activation is caused by several different mutations. Aberrations increasing E2A activity enhance the tonic BCR signaling by regulation of the BCR-related phosphatase SHP-1 (Basso and Dalla-Favera, 2015; Schmitz et al., 2012). Besides, an inactivation or missing expression of PTEN, a negative regulator of $\mathrm{PI} 3 \mathrm{~K}$, is common to increase the PI3K-AKT pathway (Lenz et al., 2008c; Schmitz et al., 2012). The main finding of our pathway analyses suggested a positive feedback loop of the PI3K-AKT pathway to the BCR to enhance its own pathway activation (Figure 4, Figure 24). However, the described negative feedback loop of the PI3K-AKT pathway could not be 
concluded from our results (Logue and Morrison, 2012). We assume that the negative feedback loop is missing or overlaid by the positive feedback loop in BLs.

Besides, no further interplays of pathways or feedback loops were found during the tonic BCR in BLs (Figure 4). One limitation could be that, except for the PI3K pathway, the pathway activations and protein phosphorylations were too weak to uncover pathway interplays. However, this is in accordance with the suggestion that the PI3K pathway is the pivotal cascade of the tonic BCR signaling (Sander et al., 2012; Schmitz et al., 2012). Therefore, it is not surprising that inhibition of the PI3K-AKT pathway reduced the cell viability of BLs whereas a MEK inhibition had no effect (Figure 5). Our findings confirmed previous analyses demonstrating the dependency of BLs on PI3K, AKT and mTOR (Schmitz et al., 2012; Spender and Inman, 2012). Only in BL-2 cells, we could not observe an effect of PI3K inhibition on cell viability. However, Schmitz et al. demonstrated that the double PI3K inhibitor concentration also reduced BL-2 cell viability after 96 hours (Schmitz et al., 2012). Furthermore, we assume that especially the positive feedback loop of the $\mathrm{PI} 3 \mathrm{~K}$ pathway to the BCR contributes to the potent and survival-reducing effect of PI3K pathway inhibitors. As negative feedback loop hamper the inhibitory effect through upregulation of AKT as it was shown in breast cancer (Logue and Morrison, 2012), we suggested that PI3K, AKT and mTOR inhibitors are considered as additional treatment strategy for BLs due to the positive feedback loop (Spender and Inman, 2014).

In conclusion, mutations of BLs contribute not only to the PI3K pathway activation but also enhance this pathway activation through a positive feedback loop probably to the BCR. As other BCR-related pathways seem to be negligible, we also assume that the PI3K pathway is the central component of the tonic BCR signaling in BLs (Figure 24). However, the exact feedback mechanism to the BCR remains to be investigated and can be achieved by phosphorylation analyses of the upstream kinases using for example mass spectrometry. In addition, it has not been elucidated yet whether mTOR or downstream signaling components are responsible for the feedback. 


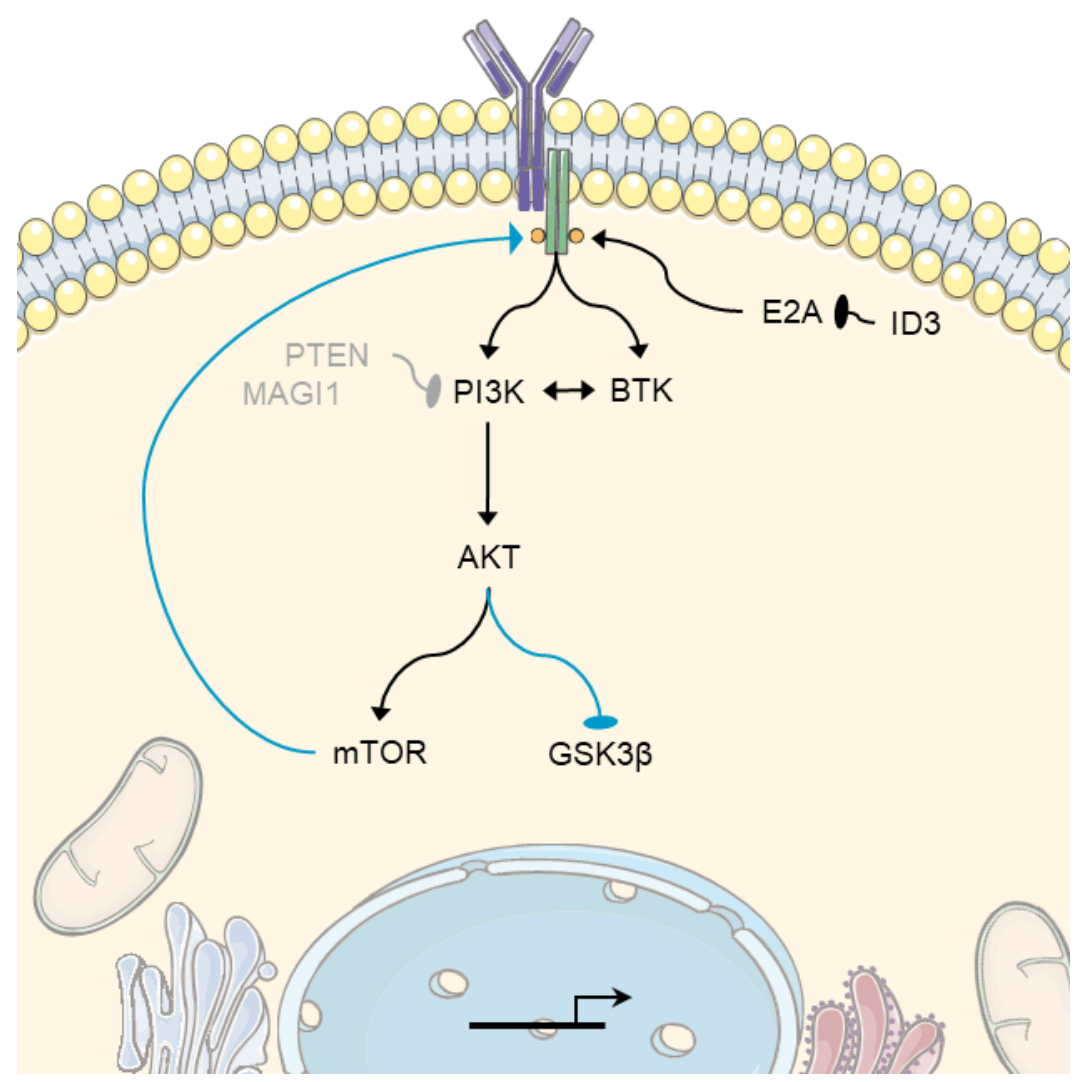

Figure 24: Proposed network scheme of pathway interplays for the tonic BCR signaling

Tonic BCR signaling in BLs includes an intrinsic induction of the PI3K pathway with a further signal transmission to AKT and mTOR. E2A activity, missing PTEN activity and a positive feedback loop downstream from mTOR to the BCR enhance the PI3K pathway. The black lines indicate the known linear signal transductions and the blue lines represent the analyzed pathway interplays and feedback loops. Proteins, which are not expressed in BL-2 cells, and the absence of pathway interactions are indicated in grey.

\subsection{BCR signaling contains crosstalk from AKT and ERK1/2 to GSK3 $\beta$}

As only the PI3K pathway was essential for the tonic BCR signaling, we additionally investigated the pathway activations after BCR stimulation in BLs. The outcome of BCR activation by antigens and induction of proximal kinases is known to increase the activity of the NF-KB, PI3K, MAPK and NFAT pathway (Kurosaki, 2011; Niiro and Clark, 2002). Our analyses confirmed the pathway activations of NF-KB, PI3K and MAPK after stimulation of the BCR with antigens (Figure 6). Interestingly, we further discovered that the positive feedback loop of the PI3K pathway was not limited to the tonic BCR signaling and was also present after receptor activation (Figure 6). As the upstream kinases were involved in the signal distribution to the different effector pathways, it was not surprising that the PI3K-AKT pathway inhibitors caused a decrease of all measured pathway activities. However, the exact mechanism and kinases which determined the signal 
distribution to the effector pathways are not fully elucidated and are still under investigation.

The PI3K-AKT pathway is not exclusively regulating its own activity. An ERK-mediated phosphorylation of MEK1 was shown to recruit and activate PTEN with subsequent negative impact on the AKT pathway (Zmajkovicova et al., 2013). Our results indicate that the activated BCR signaling contained no MEK-dependent inhibition of AKT (Figure 6). However, this could be due to mutations or absence of PTEN which is often described for BLs (Lenz et al., 2008c; Schmitz et al., 2012). Furthermore, a known interaction of AKT attenuates the MEK-ERK pathway activation by changing RAF phosphorylations (Zimmermann and Moelling, 1999). As all PI3K-AKT pathway inhibitors reduced the MEK1 and ERK1/2 phosphorylations, we could not identify a negative impact on the MEK-ERK pathway by AKT (Figure 6). Therefore, we assumed that the PI3K-AKT pathway was necessary for the MEK-ERK pathway activation during activated BCR signaling. One explanation for this pathway interplay is the regulation of an ERK-specific phosphatase by mTOR. mTOR induces the degradation of the ERK-specific phosphatase DUSP6 and therefore prolongs the ERK signaling (Bermudez et al., 2008). Nevertheless, since DUSP6 is not expressed in BL-2 cells we suggest that the decreased ERK signal was rather mediated by the positive feedback loop of the PI3K-AKT pathway to the BCR (data not shown).

Although we could not discover any interaction between the AKT and the MEK-ERK pathway in the BL cell lines, the common influence on GSK3 $\beta$ was confirmed (Figure 6). Our pathway analyses validated a reduced GSK3 $\beta$ phosphorylation through inhibition of PI3K, AKT, mTOR and MEK1/2. The influence of the AKT and MEK-ERK pathway on GSK3 $\beta$ modulates the $\beta$-catenin pathway through a double-negative feedback inducing c-MYC and cyclin D3 expression (Baracho et al., 2011; Cato et al., 2011; Ding et al., 2005; Mazzoletti et al., 2011). Therefore, the PI3K-AKT and MEK-ERK pathways were reducing GSK3 $\beta$ probably to induce cell cycle progression of BLs. Despite the fact that AKT was suggested to mediate the GSK3 $\beta$ phosphorylation, we also detected a reduced GSK3 $\beta$ phosphorylation by mTOR inhibition (Figure 6). However, this effect could be mediated by the inhibition of the positive feedback loop of the PI3K pathway as mTOR inhibition caused also reduced AKT phosphorylation.

In summary, we assume that after BCR activation the positive feedback loop of the PI3K pathway also influences the pathway activation of NF-KB and MAPKs. Although the PI3K-AKT and MEK-ERK pathway regulate GSK3 $\beta$ phosphorylation possibly to enhance cell proliferation, a direct interplay of the AKT and ERK pathway does not exist in BLs (Figure 25 left part). 


\subsection{MEK-ERK pathway is diminished by several negative feedbacks}

For cell fate decisions, the precise regulation of MAPK signal strength and duration is essential (Murphy and Blenis, 2006). Therefore, MAPKs are involved in many feedback mechanisms (Steelman et al., 2011). Due to a strong negative impact on its upstream kinases, the feedbacks of the MEK-ERK pathway were uncovered early. The common feedbacks of ERK1/2 are responsible for distinct phosphorylations of RAF as well as MEK1/2. The ERK-dependent phosphorylations of RAF-1 cause a conformation change to an inactive state (Wartmann et al., 1997), whereas the ERK-mediated MEK1/2 phosphorylation decreases the binding affinity to ERK1/2 (Lake et al., 2016). In our pathway analyses only the activating MEK1 phosphorylation were investigated. Interestingly, a strong increase in this phosphorylation was measured after MEK $1 / 2$ inhibition during the activated BCR signaling (Figure 6). This is in accordance with the well-known effect that ERK1/2 decreases the binding affinity and thus the activating phosphorylations of MEK1/2 (Lake et al., 2016). Therefore, the signal inhibition to ERK1/2 causes an increase of the active MEK1/2 phosphorylations (Fritsche-Guenther et al., 2011). As the increased MEK1 phosphorylation indicates the existence of the negative feedback loops, the ERK1/2 mediated RAF-1 phosphorylations were also investigated. The RAF-1 phosphorylation of the inactive state increased till 30 minutes after BCR activation in an ERK-dependent manner (Figure 9). While the ERK signaling is activated after few minutes, the increasing RAF-1 phosphorylation until 30 minutes further validates its ERK1/2 dependency due to the delayed induction. However, the signal duration of ERK1/2 indicates further negative regulators, as ERK1/2 activity peaks after few minutes of receptor activation followed by an intermediate activation before the signal is turned off (Figure 3) (Bluthgen, 2015). Another possibility how ERK1/2 decreases its own pathway activity could be mediated by regulation of gene expression. For instance, the gene expression of the negative regulator Sprouty2 is initiated by ERK1/2. Sprouty2 inhibits SOS and therefore the activation of RAF. However, Sprouty2 is downregulated in some lymphomas and was also not expressed in BL-2 cells (data not shown) (Frank et al., 2009). This indicates that the MEK-ERK pathway activation is quickly decreased by negative feedbacks on RAF-1 and MEK but the signal duration might be still prolonged at an intermediate activation in BLs due to the missing expression of Sprouty2.

Furthermore, the regulation of phosphatases plays a pivotal role to determine the signal strength and duration of MAPKs and to constitute pathway interplays. Several phosphatases are known to intervene at any tier of signal transduction, however some regulate especially MAPKs (Junttila et al., 2008). For instance, in lung cancer ERK induces DUSP1 after cisplatin treatment. DUSP1 in turn attenuates the pathway activity of 
p38 MAPK and JNK (Low and Zhang, 2016). However, in B cells we could not detect an ERK-dependent phosphorylation of p38 MAPK and JNK after BCR activation (Figure 6), which is in agreement with a low expression of DUSP1 in BL-2 cells (Figure 16). We assume that the interaction from ERK1/2 over DUSP1 to p38 MAPK and JNK is missing in BLs.

In addition to the positive feedback loop of the PI3K pathway, we add a negative feedback loop from ERK1/2 to RAF and MEK1/2 to the network scheme after BCR activation (Figure 25 middle part). While the positive feedback loop enhanced the PI3K pathway, the negative feedback loops of ERK diminished the MEK-ERK pathway activity after an initially peak.

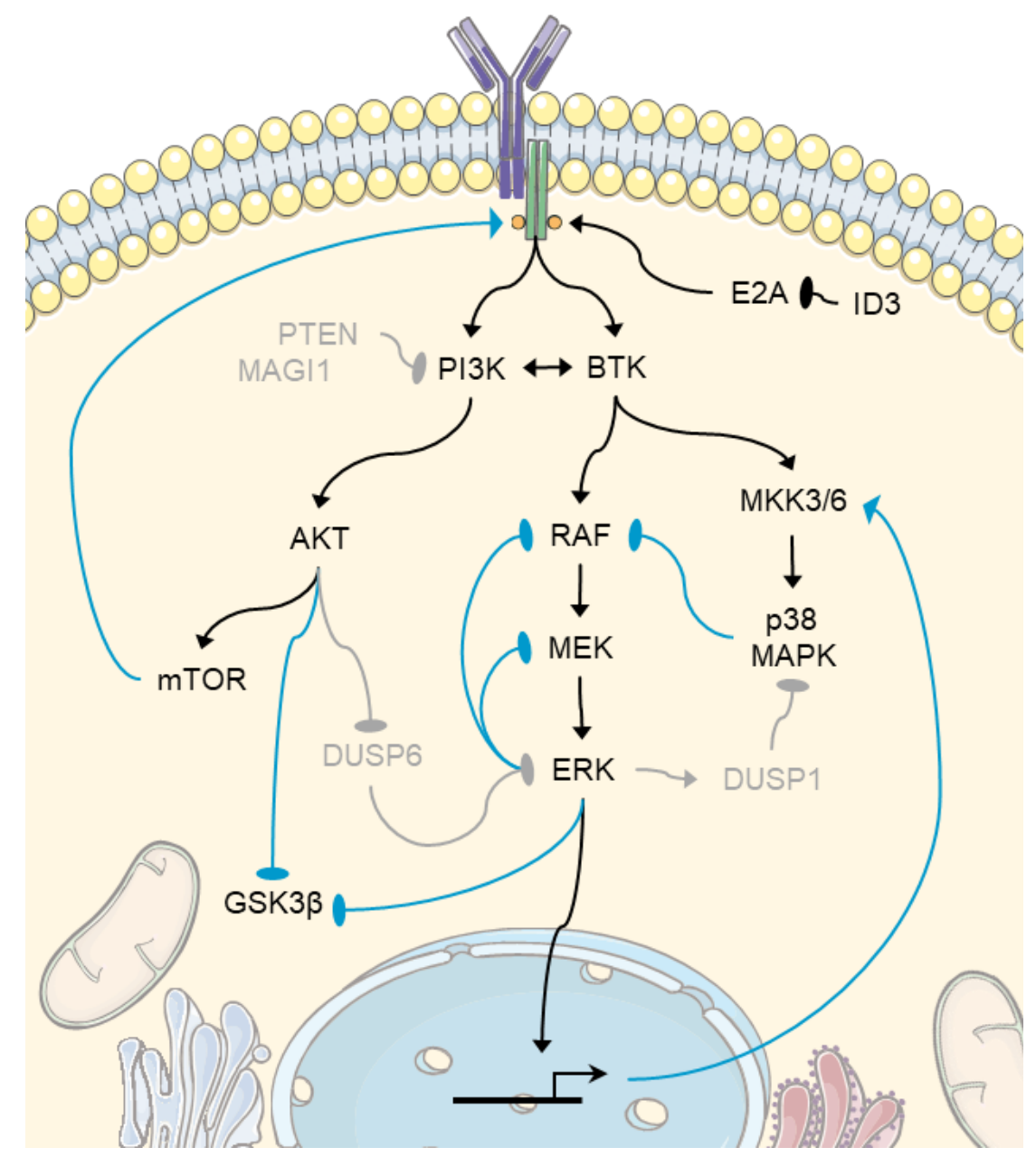

Figure 25: Proposed network scheme of pathway interplays for the activated BCR signaling BCR signaling after antigen stimulation in BLs includes the activation of the PI3K, MEK-ERK and p38 MAPK pathway. E2A activity, missing PTEN activity and a positive feedback loop downstream from mTOR to the BCR enhance the PI3K pathway. The MEK-ERK pathway reduces its own activity by negative feedback loops to its upstream kinases. p38 MAPK negatively influences the MEK-ERK pathway, while an ERK-dependent expression of MKK6 is suggested to increase p38 MAPK activation. The black lines indicate the known linear signal transductions and the blue lines represent the analyzed pathway interplays and feedback loops. Proteins, which are not expressed in BL-2 cells, and the absence of pathway interactions are indicated in grey. 


\section{4 p38 MAPK attenuates the MEK-ERK pathway}

Beside the positive feedback loop of the PI3K pathway and the negative feedback loop of the MEK-ERK pathway, the negative influence of p38 MAPK on the MEK-ERK pathway was another major finding (Figure 6). The interaction of p38 MAPK to the MEK-ERK pathway is controversially discussed and is so far not described for BLs. Due to a linear increasing RAF activity with raising p38 MAPK inhibitor concentrations higher than the concentration necessary to block p38 MAPK, the influence of p38 MAPK on the MEK-ERK pathway was referred to an unspecific inhibitor effect (Kalmes et al., 1999). However, an increasing ERK1/2 phosphorylation was also provoked by inhibition of p38 MAPK with a different inhibitor (Hirosawa et al., 2009) or by expression of a dominant negative p38 MAPK variant (Aguirre-Ghiso et al., 2001). Furthermore, our knock-down analysis of the p38a subunit caused higher ERK1/2 phosphorylations after BCR activation and thereby indicated a specific effect (Figure 8). A modulation of the MEK-ERK pathway by p38 MAPK was previously described at any kinase of the signaling cascade. In chondrocytes an immunoprecipitation of p38 MAPK and RAF-1 revealed a direct modulation of RAF-1 activity by p38 MAPK (Hutchison, 2012). Furthermore, in HL-60 cells an increased phosphorylation of RAF-1 on serine 338 was detected after p38 MAPK inhibition (Zhang et al., 2003). However, in our BL cell lines no phosphorylation changes of RAF-1 serine 338 were identified during the activated BCR signaling (Figure 9). In addition, p38 MAPK also showed no influence on the inactive state of RAF-1. As RAF-1 comprises more than 50 different phosphorylation sites, analysis of the activity could provide more insights whether p38 MAPK influences RAF-1. Furthermore, it cannot be excluded that p38 MAPK interacts with other RAF family members or upstream of RAF-1 and regulates for instance RAS activity.

Although many analyses suggested an induction of the upstream signaling compounds of MEK1/2 by p38 MAPK, a p38 MAPK-mediated activation of the phosphatase PP2A was shown to reduce the MEK1/2 phosphorylation (Wang et al., 2006). However, Wang et al. only compared the effects of a p38 MAPK inhibitor with those of a PP2A inhibitor while it was shown by others that a combinatorial inhibition still caused higher levels of phosphorylated ERK1/2 (Birkenkamp et al., 2000). Other possibilities of p38 MAPK to regulate $E R K 1 / 2$ are the stabilization of phosphorylations or the induction of phosphatases. So far, no p38 MAPK-mediated effect on ERK1/2 is described. Therefore, BL-41 cells were treated with a combination of $\mathrm{p} 38$ MAPK and MEK1/2 inhibitors to investigate a direct influence of p38 MAPK on ERK1/2 after BCR activation (Figure 6). The addition of the p38 MAPK inhibitor to the MEK inhibitor caused little more ERK1/2 phosphorylation compared to the MEK inhibitor alone. However, the ERK1/2 
phosphorylation after combinatorial treatment amounted only $20 \%$ of the ERK $1 / 2$ phosphorylation after p38 MAPK inhibition. In conclusion, we suggest that the negative interaction of p38 MAPK to the MEK-ERK pathway is upstream of MEK1/2, however, the exact mechanism remains to be elucidated (Figure 25).

Beside the ERK-mediated negative feedbacks, we assume that p38 MAPK further contributes to the fine tuning of the MEK-ERK pathway. Until now, ERK signal durations and strengths were connected to some different signaling outputs (Bluthgen, 2015), but how feedbacks influence the cellular outcomes is so far not achieved. In BLs p38 MAPK inhibition prolonged the phosphorylation of ERK $1 / 2$ but it could not be excluded that this is only due to higher initial levels of phosphorylated ERK1/2 after BCR activation (Figure 10). Besides, p38 MAPK inhibition increased the ERK1/2 activity and nuclear amounts of phosphorylated ERK1/2 after BCR activation (Figure 10). Therefore, we investigated whether p38 MAPK limits ERK1/2 target gene expression. For p38 MAPK as well as ERK1/2 more than 100 targets are described (Roskoski, 2012; Trempolec et al., 2013b). As in a previous project gene expression changes after pathway perturbations were investigated, we validated ERK1/2 target genes which were inversely regulated by p38 MAPK inhibition. Many targets were identified as ERK1/2-dependent but only MAP2K6 expression was further attenuated by p38 MAPK after BCR activation for 1 hour (Figure 14). MAP2K6 encodes the kinase MKK6, an upstream activator of p38 MAPK (Sabio and Davis, 2014). Therefore, we hypothesized that the activation of ERK1/2 upregulates MKK6 and thereby modulates p38 MAPK activation. However, the activation of MKK6 and subsequently of p38 MAPK is so far not analyzed with regard to ERK1/2 activity.

In conclusion, the activated BCR signaling contains a further negative interplay from p38 MAPK to the MEK-ERK pathway (Figure 25 right part). Thereby, we assume that p38 MAPK probably interacts with RAF-1. The attenuation of the MEK-ERK pathway by p38 MAPK reduces not only the ERK1/2 activity but also modulates ERK1/2 target gene expressions. Through the ERK-dependent expression of MKK6 an additional interaction from ERK1/2 to p38 MAPK was hypothesized. These results suggest that the balance between the MEK-ERK and p38 MAPK pathway is strongly regulated, which contributes to the pathway durations and thus determines the signaling output. 


\subsection{Feedback loops differ in ABC DLBCLs with chronic active BCR signaling}

As in BLs more pathway interplays were analyzed after BCR activation, we further investigated the pathway interactions in lymphoma cell lines with a chronic active BCR signaling. While in ABC DLBCLs no deregulations of PTEN or E2A are found, the PI3K pathway is still increased due to receptor mutations of CD79a/b (Kloo et al., 2011; Schmitz et al., 2012). These mutations and the activation with self-antigens contribute to a constitutive activation of NF-KB, ERK and NFAT (Davis et al., 2010; Young et al., 2015). In addition, NF-KB can also been activated by aberrant inductions of CARD11 or MyD88 (Lenz et al., 2008a; Ngo et al., 2011). The cell lines used for this study were selected based on different mutation profiles. HBL-1 cells carry a CD79b and MyD88 mutation, whereas OCI-LY3 cells are characterized by CARD11 and MyD88 mutations (Paul et al., 2017).

The pathway analyses after distinct perturbations in HBL-1 cells validated the discovered pathway interplays in BLs. The PI3K-AKT pathway was enhanced through a positive feedback loop, ERK1/2 negatively influenced MEK1 phosphorylation and p38 MAPK attenuated the MEK-ERK pathway (Figure 15). However, the inhibition of AKT in HBL-1 cells led to different outcomes in comparison to BL cell lines. One explanation might be that the regulation and feedbacks of the mTORC1 and mTORC2 are different compared to BLs but this hypothesis is so far not elucidated. As HBL-1 cells have no PTEN aberration, a negative influence of MEK1 along with PTEN on the PI3K pathway was expected (Zmajkovicova et al., 2013). However, no increased PI3K-AKT pathway activation was identified following MEK1/2 inhibition (Figure 15). Interestingly, in T cells it was recently discovered that the negative influence of MEK1 on PI3K required aside from PTEN the protein MAGI1 (Kozakai et al., 2018). As MAGl1 is not expressed in HBL-1 cells, this can be an explanation for the missing interplay from MEK1 to PI3K (data not shown).

Although OCI-LY3 cells are not PI3K-dependent like HBL-1 cells (Kloo et al., 2011), the positive feedback loop of the PI3K-AKT pathway was also present, however, to a lesser extent (Figure 15). This indicates that this feedback mechanism is characteristic for lymphomas even though the proliferation of OCI-LY3 cells is mediated by other pathway activations. Furthermore, the negative feedback loop of the MEK-ERK pathway was consistent in all examined cell lines, while the influence of p38 MAPK on the MEK-ERK pathway was not present in OCI-LY3 cells (Figure 15). As the absence of phosphorylation of p38 MAPK and ERK1/2 could be excluded (Figure 16), we assumed that the lack of 
interplay of p38 MAPK to the MEK-ERK pathway might be due to genetic differences and that p38 MAPK did not directly interact with RAF-1. We assume that p38 MAPK regulates rather a phosphatase or kinase and thereby influences the MEK-ERK pathway. The comparison of phosphorylation-related protein expressions led to the hypothesis that PTPN22, PTPN7, PALD1 or LCK, which were highly expressed in HBL-1 and BL-2 cells but not in OCI-LY3 cells, could mediate the interaction from p38 MAPK to the MEK-ERK pathway (Figure 16). LCK is involved in the aryl hydrocarbon receptor-mediated impairment of immunoglobulin secretion in human primary B cells (Zhou et al., 2018). In $B L$ cell lines high expression levels of LCK were reported but so far the benefit or reason is not known (Von Knethen et al., 1997). However, in T cells an interaction of p38 MAPK to ZAP70 regulates the signaling duration of the MEK-ERK pathway (Giardino Torchia et al., 2018). As ZAP70 and LCK are known to interact with each other, we hypothesize that LCK and ZAP70 probably regulate the signal duration of the MEK-ERK pathway in a p38 MAPK-dependent manner. Nevertheless, the interaction of p38 MAPK and LCK or ZAP70 as well as the ZAP70-triggered MEK-ERK pathway activation remains to be elucidated in B cell lymphomas.

In another DLBCL lymphoma cell line, DB cells, it was shown that p38 MAPK inhibition had also no impact on the ERK1/2 phosphorylation (Wojciechowski et al., 2005). Because this lymphoma cell line is a GCB DLBCL, the CARD11 mutation of OCI-LY3 cannot be responsible for the missing interplay. One similarity between OCI-LY3 and DB cells is the expression of immunoglobulin IgG, whereas the other cell lines with p38 MAPK to ERK1/2 interplay express $\lg \mathrm{M}$. Mature $\mathrm{B}$ cells express $\lg \mathrm{M}$ in a monomeric form on their cell surface prior to activation. Afterwards, B cells undergo clonal expansion and class switch recombination causing a downregulation of $\operatorname{lgM}$ and an upregulation of the $\lg \mathrm{G}$ isotype (Engels et al., 2009). The class switch occurs during the germinal center reaction and the IgM expression is essential for the proliferation of $B$ cells, while IgG expression is connected to differentiation (Rickert, 2013). Therefore, we suggest that the B cell state of origin comprises different signaling interplays and feedbacks which are reflected in B cell lymphomas.

To examine whether the different interplays of HBL-1 and OCI-LY3 cells had different cellular outcomes, the MEK1/2 and p38 MAPK inhibition was analyzed in relation to the cell viability. An impact of MEK-ERK or p38 MAPK pathway inhibition on proliferation has so far not been described. While in BLs the activation of the BCR led to a cell cycle arrest which complicates the analysis of the interplays and feedbacks with regard to proliferation (Marches et al., 1998), an increased rate of apoptosis was identified after MEK1/2 inhibition in OCI-LY3 cells (Bhalla et al., 2011). Although the same MEK1/2 inhibitor was 
used, the inhibition of MEK1/2 did not affect cell viability of OCI-LY3 cells over 72 hours (Figure 17). Furthermore, no effect on cell viability of HBL-1 and OCI-LY3 were detected after p38 MAPK or MEK1/2 inhibition. However, p38 MAPK is known to cause an increased ERK1/2 activation and tumor volume in prostate cancer (Aguirre-Ghiso et al., 2003). Although no impact on the proliferation of HBL-1 and OCI-LY3 cells was identified, further analysis regarding tumor volume, stress response or differentiation might reveal the role of p38 MAPK and ERK1/2 in B cell lymphomas.

In summary, the positive feedback loop of the PI3K pathway and the negative feedback loop of the MEK-ERK pathway seem to be conserved across the tested B cell lymphoma cell lines. The p38 MAPK influence on the MEK-ERK pathway is assumed to be lg-dependent and might even include some unknown effector proteins.

\subsection{Interplay of TLR9 and IL10R activation induces CDK2 and JNK}

In ABC DLBCLs the constitutive activation of NF-KB leads to the expression of IL6 or IL10 and to a subsequent autocrine activation of the JAK-STAT signaling (Davis et al., 2001). In the model cell line P493-6 it was shown that especially the combined activation of NF-KB and STAT3 by TLR9 and IL10R stimulation induced proliferation in a synergistic manner through expression changes of cell cycle genes (Feist et al., 2017). In addition, we showed that TLR9 and IL10R activation induced over 200 phosphorylation changes in the proteome (Figure 19). Several differences in phosphorylation were detected in the MAPK pathways (Figure 22). Therefore, we assume that the activation of MAPKs and especially JNK contributes in addition to NF-KB and STAT3 activation to the proliferative effect in the model cell line. The dependency of ABC DLBCL proliferation on JNK is already known (Gururajan et al., 2005), which further supports our hypothesis that JNK contributes to the NF-KB and STAT3-dependent proliferation of the cells. Nevertheless, the role of JNK regarding proliferation of the model cell line P493-6 after TLR9 and IL10R activation remains to be examined. Furthermore, cell cycle regulators like CDK4 were shown to be expressed after TLR9 and IL10R activation and to be essential for the proliferative effect in the model cell line P493-6 (Feist et al., 2017). Interestingly, we demonstrated that the cell cycle regulator CDK2 displayed not only higher expression levels after TLR9 and IL10R activation, but was also directly phosphorylated after receptor stimulation (Figure 22). Therefore, we suggest that the cell cycle is directly induced by TLR9 and IL10R activation and the upregulation of the cell cycle genes is only one further aspect that enhances the proliferation rate. Although a reduced proliferation in DLBCLs was already shown after CDK2 inhibition (Faber and Chiles, 2007), the explicit 
dependence of proliferation on CDK2 remains to be investigated in our model cell line P493-6.

Beside the influence on proliferation, the changed protein phosphorylations after TLR9 and IL10R activation indicated an altered cell adhesion and movement (Figure 21). For GCB DLBCLs, it was shown that modulation of cell movement resulted from Ga13 mutations (Muppidi et al., 2014). However, less is known for the migration capacity of $A B C$ DLBCLs. One recent publication investigated the migration speed in ABC DLBCLs which was enhanced by STAT3 activation (Pan et al., 2018). Our findings of IL10R activation with subsequent STAT3 activation showed no higher migration rates of the model cell line P493-6 (Figure 23). Nevertheless, we cannot exclude an influence on the migration speed. Therefore, the cell movement requires further examinations using time-lapse microscopy. In addition, the impact of NF-kB pathway activation on cell migration of $\mathrm{B}$ cell lymphomas was so far not reported. The migration rate of the model cell line P493-6 was reduced after TLR9 activation (Figure 23). Furthermore, the combined activation of TLR9 and IL10R activation showed a stronger reduction of the migration rate compared to TLR9 activation alone. This is in accordance with phosphorylation of PAK2 which was identified in our phosphoproteome analysis and is described to inactivate the protein and thereby reduce the migration capacity. Nevertheless, the TLR9 and IL10R activation led mainly to a higher phosphorylation of migration-associate proteins and it remains to be elucidated whether these phosphorylations have a negative impact on the migration of cells. Controversially, inhibition of NF-KB was reported to reduce the migration of for instance lung cancer stem cells (Zakaria et al., 2018). This emphasizes the need of further investigations to fully explain the migration capacity of the model cell line after TLR9 and IL10R activation as well as of $A B C$ DLBCLs in relation to NF-KB activation. 


\section{Summary and Conclusion}

In conclusion, this study provides a general network model of pathway interplays for B cell lymphomas. Three major interplays and feedback loops were uncovered downstream of the BCR. A positive feedback loop from mTOR or downstream targets to proximal kinases of the BCR enhances the PI3K pathway. Further investigations like phosphoproteome analyses are required to disclose the involved and affected kinases. A negative feedback loop of the MEK-ERK pathway to MEK1/2 and RAF-1 was consistently detected in all tested B cell lymphoma cell lines. While a negative influence from p38 MAPK on the MEK-ERK pathway was uncovered in B cell lymphomas expressing $\lg \mathrm{M}$, this interplay was missing in $\mathrm{lgG}$ expressing $\mathrm{B}$ cell lymphomas. To further illuminate the negative influence of p38 MAPK on the MEK-ERK pathway, analyses of the p38 MAPK interactome are necessary to uncover the affected pathway components. In addition, the validation of our findings in other lymphoma types like GCB DLBCLs, follicular lymphoma or mantle cell lymphoma can be a further aim to confirm the general network model. Nevertheless, these pathway interplays and feedback loops contribute to a better understanding and refinement of the BCR signaling network model. As pathway interplays and feedbacks alter signaling durations and strengths to achieve distinct responses to external influences, the improvement of computational network models is advantageous to predict the signaling outcome and furthermore the response of therapies. As the heterogeneity of lymphomas was shown to contain different pathway interplays, a further perspective is to include the genetic background for more accurate network models. Gene mutation and expression analyses will be useful tools to examine which pathway interplays and feedbacks occur and will hopefully provide deeper insights to improve the targeted therapies for lymphoma patients.

In the second approach, the pathway interplays after TLR9 and IL10R activation was investigated to uncover further mediators of the proliferative effect in the model cell line P493-6. Beside NF-KB and STAT3 activation, the stimulation of TLR9 and IL10R changed over 200 protein phosphorylations. A first network model of activated proteins was proposed and an impact on cell proliferation, metabolism and migration validated. Interestingly, the TLR9 and IL10R activation influence directly JNK and the cell cycle protein CDK2 to probably induce proliferation whereas the induction of proliferationassociated genes seems to be only a further aspect to increase the proliferation rate. However, a knock-out screening is required to confirm the phosphoproteom results and further possible mediators of the proliferative effect. 


\section{References}

Aguirre-Ghiso, J.A., Estrada, Y., Liu, D., and Ossowski, L. (2003). ERK(MAPK) activity as a determinant of tumor growth and dormancy; regulation by p38(SAPK). Cancer research 63, 1684-1695.

Aguirre-Ghiso, J.A., Liu, D., Mignatti, A., Kovalski, K., and Ossowski, L. (2001). Urokinase receptor and fibronectin regulate the ERK(MAPK) to p38(MAPK) activity ratios that determine carcinoma cell proliferation or dormancy in vivo. Molecular biology of the cell 12, 863-879.

Aldoss, I.T., Weisenburger, D.D., Fu, K., Chan, W.C., Vose, J.M., Bierman, P.J., Bociek, R.G., and Armitage, J.O. (2008). Adult Burkitt lymphoma: advances in diagnosis and treatment. Oncology (Williston Park) 22, 1508-1517.

Alessi, D.R., Saito, Y., Campbell, D.G., Cohen, P., Sithanandam, G., Rapp, U., Ashworth, A., Marshall, C.J., and Cowley, S. (1994). Identification of the sites in MAP kinase kinase1 phosphorylated by p74raf-1. The EMBO journal 13, 1610-1619.

Alizadeh, A.A., Eisen, M.B., Davis, R.E., Ma, C., Lossos, I.S., Rosenwald, A., Boldrick, J.C., Sabet, H., Tran, T., Yu, X., Powell, J.I., Yang, L., Marti, G.E., Moore, T., Hudson, J., Jr., Lu, L., Lewis, D.B., Tibshirani, R., Sherlock, G., Chan, W.C., Greiner, T.C., Weisenburger, D.D., Armitage, J.O., Warnke, R., Levy, R., Wilson, W., Grever, M.R., Byrd, J.C., Botstein, D., Brown, P.O., and Staudt, L.M. (2000). Distinct types of diffuse large B-cell lymphoma identified by gene expression profiling. Nature 403, 503-511.

Arlt, A. (2018). Hodgkin lymphoma secreted factors determine macrophage polarization and function. In University Medical Centre Goettingen (http://hdl.handle.net/11858/001735-0000-002E-E4A8-9: Georg-August-University Goettingen).

Ashwell, J.D. (2006). The many paths to p38 mitogen-activated protein kinase activation in the immune system. Nature reviews Immunology 6, 532-540.

Avalos, A.M., and Ploegh, H.L. (2014). Early BCR Events and Antigen Capture, Processing, and Loading on MHC Class II on B Cells. Frontiers in immunology 5, 1-5.

Bain, J., Plater, L., Elliott, M., Shpiro, N., Hastie, C.J., McLauchlan, H., Klevernic, I., Arthur, J.S., Alessi, D.R., and Cohen, P. (2007). The selectivity of protein kinase inhibitors: a further update. The Biochemical journal 408, 297-315.

Baracho, G.V., Miletic, A.V., Omori, S.A., Cato, M.H., and Rickert, R.C. (2011). Emergence of the PI3-kinase pathway as a central modulator of normal and aberrant $\mathrm{B}$ cell differentiation. Current opinion in immunology 23, 178-183.

Basso, K., and Dalla-Favera, R. (2015). Germinal centres and B cell lymphomagenesis. Nature reviews Immunology 15, 172-184.

Beguelin, W., Popovic, R., Teater, M., Jiang, Y., Bunting, K.L., Rosen, M., Shen, H., Yang, S.N., Wang, L., Ezponda, T., Martinez-Garcia, E., Zhang, H., Zheng, Y., Verma, S.K., McCabe, M.T., Ott, H.M., Van Aller, G.S., Kruger, R.G., Liu, Y., McHugh, C.F., Scott, D.W., Chung, Y.R., Kelleher, N., Shaknovich, R., Creasy, C.L., Gascoyne, R.D., Wong, K.K., Cerchietti, L., Levine, R.L., Abdel-Wahab, O., Licht, J.D., Elemento, O., and Melnick, A.M. (2013). EZH2 is required for germinal center formation and somatic EZH2 mutations promote lymphoid transformation. Cancer cell 23, 677-692. 
Bermudez, O., Marchetti, S., Pages, G., and Gimond, C. (2008). Post-translational regulation of the ERK phosphatase DUSP6/MKP3 by the mTOR pathway. Oncogene 27, 3685-3691.

Bertrand, S., Berger, R., Philip, T., Bernheim, A., Bryon, P.A., Bertoglio, J., Dore, J.F., Brunat-Mentigny, M., and Lenoir, G.M. (1981). Variant translocation in a non endemic case of Burkitt's lymphoma: $t(8 ; 22)$ in an Epstein--Barr virus negative tumour and in a derived cell line. Eur J Cancer 17, 577-584.

Bhalla, S., Evens, A.M., Dai, B., Prachand, S., Gordon, L.I., and Gartenhaus, R.B. (2011). The novel anti-MEK small molecule AZD6244 induces BIM-dependent and AKTindependent apoptosis in diffuse large B-cell lymphoma. Blood 118, 1052-1061.

Birkenkamp, K.U., Tuyt, L.M., Lummen, C., Wierenga, A.T., Kruijer, W., and Vellenga, E. (2000). The p38 MAP kinase inhibitor SB203580 enhances nuclear factor-kappa B transcriptional activity by a non-specific effect upon the ERK pathway. British journal of pharmacology 131, 99-107.

Blachly, J.S., and Baiocchi, R.A. (2014). Targeting PI3-kinase (PI3K), AKT and mTOR axis in lymphoma. British journal of haematology 167, 19-32.

Blum, K.A., Lozanski, G., and Byrd, J.C. (2004). Adult Burkitt leukemia and lymphoma. Blood 104, 3009-3020.

Bluthgen, N. (2015). Signaling output: it's all about timing and feedbacks. Molecular systems biology 11, 1-2.

Boyd, S.D., Natkunam, Y., Allen, J.R., and Warnke, R.A. (2013). Selective immunophenotyping for diagnosis of B-cell neoplasms: immunohistochemistry and flow cytometry strategies and results. Appl Immunohistochem Mol Morphol 21, 116-131.

Brunet, A., Bonni, A., Zigmond, M.J., Lin, M.Z., Juo, P., Hu, L.S., Anderson, M.J., Arden, K.C., Blenis, J., and Greenberg, M.E. (1999). Akt promotes cell survival by phosphorylating and inhibiting a Forkhead transcription factor. Cell 96, 857-868.

Brunet, A., Pages, G., and Pouyssegur, J. (1994). Growth factor-stimulated MAP kinase induces rapid retrophosphorylation and inhibition of MAP kinase kinase (MEK1). FEBS Lett 346, 299-303.

Burkitt, D. (1958). A sarcoma involving the jaws in African children. The British journal of surgery $46,218-223$.

Cato, M.H., Chintalapati, S.K., Yau, I.W., Omori, S.A., and Rickert, R.C. (2011). Cyclin D3 is selectively required for proliferative expansion of germinal center $B$ cells. Molecular and cellular biology $31,127-137$.

Challa-Malladi, M., Lieu, Y.K., Califano, O., Holmes, A.B., Bhagat, G., Murty, V.V., Dominguez-Sola, D., Pasqualucci, L., and Dalla-Favera, R. (2011). Combined genetic inactivation of beta2-Microglobulin and CD58 reveals frequent escape from immune recognition in diffuse large $B$ cell lymphoma. Cancer cell 20, 728-740.

Chen, H.C. (2005). Boyden chamber assay. Methods Mol Biol 294, 15-22.

Chen, R.H., Sarnecki, C., and Blenis, J. (1992). Nuclear localization and regulation of erkand rsk-encoded protein kinases. Molecular and cellular biology 12, 915-927. 
Compagno, M., Lim, W.K., Grunn, A., Nandula, S.V., Brahmachary, M., Shen, Q., Bertoni, F., Ponzoni, M., Scandurra, M., Califano, A., Bhagat, G., Chadburn, A., Dalla-Favera, R., and Pasqualucci, L. (2009). Mutations of multiple genes cause deregulation of NF-kappaB in diffuse large B-cell lymphoma. Nature 459, 717-721.

Corbalan-Garcia, S., Yang, S.S., Degenhardt, K.R., and Bar-Sagi, D. (1996). Identification of the mitogen-activated protein kinase phosphorylation sites on human Sos1 that regulate interaction with Grb2. Molecular and cellular biology 16, 5674-5682.

Corso, J., Pan, K.T., Walter, R., Doebele, C., Mohr, S., Bohnenberger, H., Strobel, P., Lenz, C., Slabicki, M., Hullein, J., Comoglio, F., Rieger, M.A., Zenz, T., Wienands, J., Engelke, M., Serve, H., Urlaub, H., and Oellerich, T. (2016). Elucidation of tonic and activated B-cell receptor signaling in Burkitt's lymphoma provides insights into regulation of cell survival. Proceedings of the National Academy of Sciences of the United States of America 113, 5688-5693.

Cox, J., and Mann, M. (2008). MaxQuant enables high peptide identification rates, individualized p.p.b.-range mass accuracies and proteome-wide protein quantification. Nature biotechnology 26, 1367-1372.

Cuenda, A., and Rousseau, S. (2007). p38 MAP-kinases pathway regulation, function and role in human diseases. Biochimica et biophysica acta 1773, 1358-1375.

Davis, R.E., Brown, K.D., Siebenlist, U., and Staudt, L.M. (2001). Constitutive nuclear factor kappaB activity is required for survival of activated B cell-like diffuse large B cell lymphoma cells. The Journal of experimental medicine 194, 1861-1874.

Davis, R.E., Ngo, V.N., Lenz, G., Tolar, P., Young, R.M., Romesser, P.B., Kohlhammer, H., Lamy, L., Zhao, H., Yang, Y., Xu, W., Shaffer, A.L., Wright, G., Xiao, W., Powell, J., Jiang, J.K., Thomas, C.J., Rosenwald, A., Ott, G., Muller-Hermelink, H.K., Gascoyne, R.D., Connors, J.M., Johnson, N.A., Rimsza, L.M., Campo, E., Jaffe, E.S., Wilson, W.H., Delabie, J., Smeland, E.B., Fisher, R.I., Braziel, R.M., Tubbs, R.R., Cook, J.R., Weisenburger, D.D., Chan, W.C., Pierce, S.K., and Staudt, L.M. (2010). Chronic active Bcell-receptor signalling in diffuse large B-cell lymphoma. Nature 463, 88-92.

Deeb, S.J., Tyanova, S., Hummel, M., Schmidt-Supprian, M., Cox, J., and Mann, M. (2015). Machine Learning-based Classification of Diffuse Large B-cell Lymphoma Patients by Their Protein Expression Profiles. Molecular \& cellular proteomics : MCP 14, 29472960.

Deshmukh, A.S., Murgia, M., Nagaraj, N., Treebak, J.T., Cox, J., and Mann, M. (2015). Deep proteomics of mouse skeletal muscle enables quantitation of protein isoforms, metabolic pathways, and transcription factors. Molecular \& cellular proteomics : MCP 14, 841-853.

Dhillon, A.S., Hagan, S., Rath, O., and Kolch, W. (2007). MAP kinase signalling pathways in cancer. Oncogene 26, 3279-3290.

Ding, B.B., Yu, J.J., Yu, R.Y., Mendez, L.M., Shaknovich, R., Zhang, Y., Cattoretti, G., and Ye, B.H. (2008). Constitutively activated STAT3 promotes cell proliferation and survival in the activated B-cell subtype of diffuse large B-cell lymphomas. Blood 111, 1515-1523.

Ding, H., Gabali, A.M., Jenson, S.D., Lim, M.S., and Elenitoba-Johnson, K.S. (2009). P38 mitogen activated protein kinase expression and regulation by interleukin-4 in human $B$ cell non-Hodgkin lymphomas. J Hematop 2, 195-204. 
Ding, Q., Xia, W., Liu, J.C., Yang, J.Y., Lee, D.F., Xia, J., Bartholomeusz, G., Li, Y., Pan, Y., Li, Z., Bargou, R.C., Qin, J., Lai, C.C., Tsai, F.J., Tsai, C.H., and Hung, M.C. (2005). Erk associates with and primes GSK-3beta for its inactivation resulting in upregulation of beta-catenin. Mol Cell 19, 159-170.

Dorel, M., Klinger, B., Gross, T., Sieber, A., Prahallad, A., Bosdriesz, E., Wessels, L.F.A., and Bluthgen, N. (2018). Modelling signalling networks from perturbation data. Bioinformatics 34, 4079-4086.

Dougherty, M.K., Muller, J., Ritt, D.A., Zhou, M., Zhou, X.Z., Copeland, T.D., Conrads, T.P., Veenstra, T.D., Lu, K.P., and Morrison, D.K. (2005). Regulation of Raf-1 by direct feedback phosphorylation. Mol Cell 17, 215-224.

Engels, N., Konig, L.M., Heemann, C., Lutz, J., Tsubata, T., Griep, S., Schrader, V., and Wienands, J. (2009). Recruitment of the cytoplasmic adaptor Grb2 to surface IgG and IgE provides antigen receptor-intrinsic costimulation to class-switched B cells. Nature immunology 10, 1018-1025.

Faber, A.C., and Chiles, T.C. (2007). Inhibition of cyclin-dependent kinase-2 induces apoptosis in human diffuse large B-cell lymphomas. Cell Cycle 6, 2982-2989.

Fabre-Guillevin, E., Tabrizi, R., Coulon, V., Monnereau, A., Eghbali, H., Soubeyran, I., and Soubeyran, P. (2006). Aggressive non-Hodgkin's lymphoma: concomitant evaluation of interleukin-2, soluble interleukin-2 receptor, interleukin-4, interleukin-6, interleukin-10 and correlation with outcome. Leukemia \& lymphoma 47, 603-611.

Feist, M. (2016). Synergism of IL10R and TLR9 signaling affects gene expression, proliferation and metabolism in B cells: A comparative study of STAT3/NF-kB and c-Myc mediated effects. In University Medical Centre Goettingen (http://hdl.handle.net/11858/001735-0000-002B-7C1F-1: Georg-August-University Goettingen).

Feist, M., Kemper, J., Taruttis, F., Rehberg, T., Engelmann, J.C., Gronwald, W., Hummel, M., Spang, R., and Kube, D. (2017). Synergy of interleukin 10 and toll-like receptor 9 signalling in B cell proliferation: Implications for lymphoma pathogenesis. International journal of cancer 140, 1147-1158.

Feist, M., Schwarzfischer, P., Heinrich, P., Sun, X., Kemper, J., von Bonin, F., PerezRubio, P., Taruttis, F., Rehberg, T., Dettmer, K., Gronwald, W., Reinders, J., Engelmann, J.C., Dudek, J., Klapper, W., Trumper, L., Spang, R., Oefner, P.J., and Kube, D. (2018). Cooperative STAT/NF-kappaB signaling regulates lymphoma metabolic reprogramming and aberrant GOT2 expression. Nat Commun 9, 1514.

Feng, Y., Wen, J., and Chang, C.C. (2009). p38 Mitogen-activated protein kinase and hematologic malignancies. Archives of pathology \& laboratory medicine 133, 1850-1856.

Frank, M.J., Dawson, D.W., Bensinger, S.J., Hong, J.S., Knosp, W.M., Xu, L., Balatoni, C.E., Allen, E.L., Shen, R.R., Bar-Sagi, D., Martin, G.R., and Teitell, M.A. (2009). Expression of sprouty2 inhibits B-cell proliferation and is epigenetically silenced in mouse and human B-cell lymphomas. Blood 113, 2478-2487.

Fritsche-Guenther, R., Witzel, F., Sieber, A., Herr, R., Schmidt, N., Braun, S., Brummer, T., Sers, C., and Bluthgen, N. (2011). Strong negative feedback from Erk to Raf confers robustness to MAPK signalling. Molecular systems biology 7, 1-13. 
Fukunaga, R., and Hunter, T. (1997). MNK1, a new MAP kinase-activated protein kinase, isolated by a novel expression screening method for identifying protein kinase substrates. The EMBO journal 16, 1921-1933.

Giardino Torchia, M.L., Dutta, D., Mittelstadt, P.R., Guha, J., Gaida, M.M., Fish, K., Barr, V.A., Akpan, I.O., Samelson, L.E., Tagad, H.D., Debnath, S., Miller Jenkins, L.M., Appella, E., and Ashwell, J.D. (2018). Intensity and duration of TCR signaling is limited by p38 phosphorylation of ZAP-70(T293) and destabilization of the signalosome. Proceedings of the National Academy of Sciences of the United States of America 115, 2174-2179.

Gupta, M., Han, J.J., Stenson, M., Maurer, M., Wellik, L., Hu, G., Ziesmer, S., Dogan, A., and Witzig, T.E. (2012). Elevated serum IL-10 levels in diffuse large B-cell lymphoma: a mechanism of aberrant JAK2 activation. Blood 119, 2844-2853.

Gururajan, M., Chui, R., Karuppannan, A.K., Ke, J., Jennings, C.D., and Bondada, S. (2005). C-Jun N-terminal kinase (JNK) is required for survival and proliferation of Blymphoma cells. Blood 106, 1382-1391.

Haan, C., and Behrmann, I. (2007). A cost effective non-commercial ECL-solution for Western blot detections yielding strong signals and low background. J Immunol Methods $318,11-19$.

Hanafusa, H., Torii, S., Yasunaga, T., and Nishida, E. (2002). Sprouty1 and Sprouty2 provide a control mechanism for the Ras/MAPK signalling pathway. Nat Cell Biol 4, 850858.

Hand, E. (2013). Dissecting and Modeling Oncogene Dependent Molecular Mechanisms in Lymphoma Genesis and Progression. In University Medical Centre Goettingen (http://hdl.handle.net/11858/00-1735-0000-0001-BC55-E: Georg-August-University Goettingen).

Hendriks, R.W., Yuvaraj, S., and Kil, L.P. (2014). Targeting Bruton's tyrosine kinase in B cell malignancies. Nature reviews Cancer 14, 219-232.

Hirosawa, M., Nakahara, M., Otosaka, R., Imoto, A., Okazaki, T., and Takahashi, S. (2009). The p38 pathway inhibitor SB202190 activates MEK/MAPK to stimulate the growth of leukemia cells. Leuk Res 33, 693-699.

Huang da, W., Sherman, B.T., and Lempicki, R.A. (2009). Systematic and integrative analysis of large gene lists using DAVID bioinformatics resources. Nat Protoc 4, 44-57.

Hutchison, M.R. (2012). BDNF alters ERK/p38 MAPK activity ratios to promote differentiation in growth plate chondrocytes. Mol Endocrinol 26, 1406-1416.

Ihle, J.N. (1995). The Janus protein tyrosine kinases in hematopoietic cytokine signaling. Semin Immunol 7, 247-254.

Jeffrey, K.L., Camps, M., Rommel, C., and Mackay, C.R. (2007). Targeting dual-specificity phosphatases: manipulating MAP kinase signalling and immune responses. Nature reviews Drug discovery 6, 391-403.

Jiang, A., Craxton, A., Kurosaki, T., and Clark, E.A. (1998). Different Protein Tyrosine Kinases Are Required for B Cell Antigen Receptor-mediated Activation of Extracellular Signal-Regulated kinase, c-Jun $\mathrm{NH}<$ sub $>2</$ sub $>$-terminal Kinase 1, and p38 Mitogenactivated Protein Kinase. The Journal of experimental medicine 188, 1297-1306. 
Jiang, X., Guo, H., Wu, J., He, Q., Li, Y., Wang, M., Pan, H., Li, W., Wang, J., Wang, Q., Shen, J., Ke, Y., and Zhou, R. (2014). Critical role of SHP2 (PTPN11) signaling in germinal center-derived lymphoma. Haematologica 99, 1834-1845.

Jin, G., Hamaguchi, Y., Matsushita, T., Hasegawa, M., Le Huu, D., Ishiura, N., Naka, K., Hirao, A., Takehara, K., and Fujimoto, M. (2013). B-cell linker protein expression contributes to controlling allergic and autoimmune diseases by mediating IL-10 production in regulatory B cells. The Journal of allergy and clinical immunology 131, 1674-1682.

Jost, P.J., and Ruland, J. (2007). Aberrant NF-kappaB signaling in lymphoma: mechanisms, consequences, and therapeutic implications. Blood 109, 2700-2707.

Junttila, M.R., Li, S.P., and Westermarck, J. (2008). Phosphatase-mediated crosstalk between MAPK signaling pathways in the regulation of cell survival. FASEB journal : official publication of the Federation of American Societies for Experimental Biology 22, 954-965.

Kalmes, A., Deou, J., Clowes, A.W., and Daum, G. (1999). Raf-1 is activated by the p38 mitogen-activated protein kinase inhibitor, SB203580. FEBS Letters 444, 71-74.

Kelemen, K., Braziel, R.M., Gatter, K., Bakke, T.C., Olson, S., and Fan, G. (2010). Immunophenotypic variations of Burkitt lymphoma. Am J Clin Pathol 134, 127-138.

Klapproth, K., and Wirth, T. (2010). Advances in the understanding of MYC-induced lymphomagenesis. British journal of haematology 149, 484-497.

Klein, U., and Dalla-Favera, R. (2008). Germinal centres: role in B-cell physiology and malignancy. Nature reviews Immunology 8, 22-33.

Klinger, B., Sieber, A., Fritsche-Guenther, R., Witzel, F., Berry, L., Schumacher, D., Yan, Y., Durek, P., Merchant, M., Schafer, R., Sers, C., and Bluthgen, N. (2013). Network quantification of EGFR signaling unveils potential for targeted combination therapy. Molecular systems biology 9, 1-14.

Kloo, B., Nagel, D., Pfeifer, M., Grau, M., Duwel, M., Vincendeau, M., Dorken, B., Lenz, P., Lenz, G., and Krappmann, D. (2011). Critical role of PI3K signaling for NF-kappaBdependent survival in a subset of activated B-cell-like diffuse large B-cell lymphoma cells. Proceedings of the National Academy of Sciences of the United States of America 108, 272-277.

Kozakai, T., Takahashi, M., Higuchi, M., Hara, T., Saito, K., Tanaka, Y., Masuko, M., Takizawa, J., Sone, H., and Fujii, M. (2018). MAGl-1 expression is decreased in several types of human T-cell leukemia cell lines, including adult T-cell leukemia. Int $\mathrm{J}$ Hematol 107, 337-344.

Kuppers, R. (2005). Mechanisms of B-cell lymphoma pathogenesis. Nature reviews Cancer 5, 251-262.

Kurosaki, T. (1999). Genetic analysis of B cell antigen receptor signaling. Annu Rev Immunol 17, 555-592.

Kurosaki, T. (2011). Regulation of BCR signaling. Molecular immunology 48, 1287-1291.

Lachmann, A., and Ma'ayan, A. (2009). KEA: kinase enrichment analysis. Bioinformatics 25, 684-686. 
Laemmli, U.K. (1970). Cleavage of structural proteins during the assembly of the head of bacteriophage T4. Nature 227, 680-685.

Lake, D., Correa, S.A., and Muller, J. (2016). Negative feedback regulation of the ERK1/2 MAPK pathway. Cellular and molecular life sciences : CMLS 73, 4397-4413.

Lam, K.P., Kuhn, R., and Rajewsky, K. (1997). In vivo ablation of surface immunoglobulin on mature $B$ cells by inducible gene targeting results in rapid cell death. Cell 90, 10731083.

Lam, L.T., Wright, G., Davis, R.E., Lenz, G., Farinha, P., Dang, L., Chan, J.W., Rosenwald, A., Gascoyne, R.D., and Staudt, L.M. (2008). Cooperative signaling through the signal transducer and activator of transcription 3 and nuclear factor-\{kappa\}B pathways in subtypes of diffuse large B-cell lymphoma. Blood 111, 3701-3713.

Laplante, M., and Sabatini, D.M. (2012). mTOR signaling in growth control and disease. Cell 149, 274-293.

Lech-Maranda, E., Bienvenu, J., Michallet, A.S., Houot, R., Robak, T., Coiffier, B., and Salles, G. (2006). Elevated IL-10 plasma levels correlate with poor prognosis in diffuse large B-cell lymphoma. Eur Cytokine Netw 17, 60-66.

Lenoir, G.M., Vuillaume, M., and Bonnardel, C. (1985). The use of lymphomatous and lymphoblastoid cell lines in the study of Burkitt's lymphoma. IARC scientific publications, 309-318.

Lenz, G., Davis, R.E., Ngo, V.N., Lam, L., George, T.C., Wright, G.W., Dave, S.S., Zhao, H., Xu, W., Rosenwald, A., Ott, G., Muller-Hermelink, H.K., Gascoyne, R.D., Connors, J.M., Rimsza, L.M., Campo, E., Jaffe, E.S., Delabie, J., Smeland, E.B., Fisher, R.I., Chan, W.C., and Staudt, L.M. (2008a). Oncogenic CARD11 mutations in human diffuse large B cell lymphoma. Science 319, 1676-1679.

Lenz, G., Wright, G., Dave, S.S., Xiao, W., Powell, J., Zhao, H., Xu, W., Tan, B., Goldschmidt, N., labal, J., Vose, J., Bast, M., Fu, K., Weisenburger, D.D., Greiner, T.C., Armitage, J.O., Kyle, A., May, L., Gascoyne, R.D., Connors, J.M., Troen, G., Holte, H., Kvaloy, S., Dierickx, D., Verhoef, G., Delabie, J., Smeland, E.B., Jares, P., Martinez, A., Lopez-Guillermo, A., Montserrat, E., Campo, E., Braziel, R.M., Miller, T.P., Rimsza, L.M., Cook, J.R., Pohlman, B., Sweetenham, J., Tubbs, R.R., Fisher, R.I., Hartmann, E., Rosenwald, A., Ott, G., Muller-Hermelink, H.K., Wrench, D., Lister, T.A., Jaffe, E.S., Wilson, W.H., Chan, W.C., and Staudt, L.M. (2008b). Stromal gene signatures in large-Bcell lymphomas. The New England journal of medicine 359, 2313-2323.

Lenz, G., Wright, G.W., Emre, N.C., Kohlhammer, H., Dave, S.S., Davis, R.E., Carty, S., Lam, L.T., Shaffer, A.L., Xiao, W., Powell, J., Rosenwald, A., Ott, G., Muller-Hermelink, H.K., Gascoyne, R.D., Connors, J.M., Campo, E., Jaffe, E.S., Delabie, J., Smeland, E.B., Rimsza, L.M., Fisher, R.I., Weisenburger, D.D., Chan, W.C., and Staudt, L.M. (2008c). Molecular subtypes of diffuse large B-cell lymphoma arise by distinct genetic pathways. Proceedings of the National Academy of Sciences of the United States of America 105, 13520-13525.

Leonard, W.J., and Lin, J.X. (2000). Cytokine receptor signaling pathways. The Journal of allergy and clinical immunology 105, 877-888.

Levy, D.E., and Darnell, J.E., Jr. (2002). Stats: transcriptional control and biological impact. Nature reviews Molecular cell biology 3, 651-662. 
Limon, J.J., and Fruman, D.A. (2012). Akt and mTOR in B Cell Activation and Differentiation. Frontiers in immunology 3, 1-12.

Logue, J.S., and Morrison, D.K. (2012). Complexity in the signaling network: insights from the use of targeted inhibitors in cancer therapy. Genes Dev 26, 641-650.

Love, C., Sun, Z., Jima, D., Li, G., Zhang, J., Miles, R., Richards, K.L., Dunphy, C.H., Choi, W.W., Srivastava, G., Lugar, P.L., Rizzieri, D.A., Lagoo, A.S., Bernal-Mizrachi, L., Mann, K.P., Flowers, C.R., Naresh, K.N., Evens, A.M., Chadburn, A., Gordon, L.I., Czader, M.B., Gill, J.I., Hsi, E.D., Greenough, A., Moffitt, A.B., McKinney, M., Banerjee, A., Grubor, V., Levy, S., Dunson, D.B., and Dave, S.S. (2012). The genetic landscape of mutations in Burkitt lymphoma. Nat Genet 44, 1321-1325.

Low, H.B., and Zhang, Y. (2016). Regulatory Roles of MAPK Phosphatases in Cancer. Immune network 16, 85-98.

MacLennan, I.C. (1994). Germinal centers. Annu Rev Immunol 12, 117-139.

Magrath, I.T., Pizzo, P.A., Whang-Peng, J., Douglass, E.C., Alabaster, O., Gerber, P., Freeman, C.B., and Novikovs, L. (1980). Characterization of lymphoma-derived cell lines: comparison of cell lines positive and negative for Epstein-Barr virus nuclear antigen. I. Physical, cytogenetic, and growth characteristics. Journal of the National Cancer Institute 64, 465-476.

Mandelbaum, J., Bhagat, G., Tang, H., Mo, T., Brahmachary, M., Shen, Q., Chadburn, A., Rajewsky, K., Tarakhovsky, A., Pasqualucci, L., and Dalla-Favera, R. (2010). BLIMP1 is a tumor suppressor gene frequently disrupted in activated B cell-like diffuse large B cell lymphoma. Cancer cell 18, 568-579.

Mann, M. (2006). Functional and quantitative proteomics using SILAC. Nature reviews Molecular cell biology 7, 952-958.

Marches, R., Scheuermann, R.H., and Uhr, J.W. (1998). Cancer dormancy: role of cyclindependent kinase inhibitors in induction of cell cycle arrest mediated via membrane lgM. Cancer research 58, 691-697.

Mazzoletti, M., Bortolin, F., Brunelli, L., Pastorelli, R., Di Giandomenico, S., Erba, E., Ubezio, P., and Broggini, M. (2011). Combination of PI3K/mTOR inhibitors: antitumor activity and molecular correlates. Cancer research 71, 4573-4584.

Molyneux, E.M., Rochford, R., Griffin, B., Newton, R., Jackson, G., Menon, G., Harrison, C.J., Israels, T., and Bailey, S. (2012). Burkitt's lymphoma. Lancet 379, 1234-1244.

Muppidi, J.R., Lu, E., and Cyster, J.G. (2015). The G protein-coupled receptor P2RY8 and follicular dendritic cells promote germinal center confinement of $B$ cells, whereas S1PR3 can contribute to their dissemination. The Journal of experimental medicine 212, 22132222.

Muppidi, J.R., Schmitz, R., Green, J.A., Xiao, W., Larsen, A.B., Braun, S.E., An, J., Xu, Y., Rosenwald, A., Ott, G., Gascoyne, R.D., Rimsza, L.M., Campo, E., Jaffe, E.S., Delabie, J., Smeland, E.B., Braziel, R.M., Tubbs, R.R., Cook, J.R., Weisenburger, D.D., Chan, W.C., Vaidehi, N., Staudt, L.M., and Cyster, J.G. (2014). Loss of signalling via Galpha13 in germinal centre B-cell-derived lymphoma. Nature 516, 254-258.

Murphy, L.O., and Blenis, J. (2006). MAPK signal specificity: the right place at the right time. Trends Biochem Sci 31, 268-275. 
Natkunam, Y. (2007). The biology of the germinal center. Hematology American Society of Hematology Education Program, 210-215.

Ngo, V.N., Young, R.M., Schmitz, R., Jhavar, S., Xiao, W., Lim, K.H., Kohlhammer, H., Xu, W., Yang, Y., Zhao, H., Shaffer, A.L., Romesser, P., Wright, G., Powell, J., Rosenwald, A., Muller-Hermelink, H.K., Ott, G., Gascoyne, R.D., Connors, J.M., Rimsza, L.M., Campo, E., Jaffe, E.S., Delabie, J., Smeland, E.B., Fisher, R.I., Braziel, R.M., Tubbs, R.R., Cook, J.R., Weisenburger, D.D., Chan, W.C., and Staudt, L.M. (2011). Oncogenically active MYD88 mutations in human lymphoma. Nature 470, 115-119.

Niiro, H., and Clark, E.A. (2002). Regulation of B-cell fate by antigen-receptor signals. Nature reviews Immunology 2, 945-956.

Nozawa, Y., Abe, M., Wakasa, H., Ohno, H., Fukuhara, S., Kinoshita, T., and Osato, T. (1988). Establishment and characterization of an Epstein-Barr virus negative B-cell lymphoma cell line and successful heterotransplantation. The Tohoku journal of experimental medicine 156, 319-330.

Ogasawara, T., Yasuyama, M., and Kawauchi, K. (2003). Constitutive activation of extracellular signal-regulated kinase and p38 mitogen-activated protein kinase in B-cell lymphoproliferative disorders. Int J Hematol 77, 364-370.

Oh-hora, M., Johmura, S., Hashimoto, A., Hikida, M., and Kurosaki, T. (2003). Requirement for Ras guanine nucleotide releasing protein 3 in coupling phospholipase Cgamma2 to Ras in B cell receptor signaling. The Journal of experimental medicine 198, 1841-1851.

Okada, T., Maeda, A., Iwamatsu, A., Gotoh, K., and Kurosaki, T. (2000). BCAP: the tyrosine kinase substrate that connects $B$ cell receptor to phosphoinositide 3-kinase activation. Immunity 13, 817-827.

Olsen, J.V., Blagoev, B., Gnad, F., Macek, B., Kumar, C., Mortensen, P., and Mann, M. (2006). Global, in vivo, and site-specific phosphorylation dynamics in signaling networks. Cell 127, 635-648.

Ott, G., Rosenwald, A., and Campo, E. (2013). Understanding MYC-driven aggressive Bcell lymphomas: pathogenesis and classification. Blood 122, 3884-3891.

Ott, G., Ziepert, M., Klapper, W., Horn, H., Szczepanowski, M., Bernd, H.W., Thorns, C., Feller, A.C., Lenze, D., Hummel, M., Stein, H., Muller-Hermelink, H.K., Frank, M., Hansmann, M.L., Barth, T.F., Moller, P., Cogliatti, S., Pfreundschuh, M., Schmitz, N., Trumper, L., Loeffler, M., and Rosenwald, A. (2010). Immunoblastic morphology but not the immunohistochemical GCB/nonGCB classifier predicts outcome in diffuse large B-cell lymphoma in the RICOVER-60 trial of the DSHNHL. Blood 116, 4916-4925.

Ozaki, K., Kadomoto, R., Asato, K., Tanimura, S., Itoh, N., and Kohno, M. (2001). ERK pathway positively regulates the expression of Sprouty genes. Biochem Biophys Res Commun 285, 1084-1088.

Pan, Y.R., Chen, C.C., Chan, Y.T., Wang, H.J., Chien, F.T., Chen, Y.L., Liu, J.L., and Yang, M.H. (2018). STAT3-coordinated migration facilitates the dissemination of diffuse large B-cell lymphomas. Nat Commun 9, 1-16. 
Pao, L.I., Famiglietti, S.J., and Cambier, J.C. (1998). Asymmetrical phosphorylation and function of immunoreceptor tyrosine-based activation motif tyrosines in B cell antigen receptor signal transduction. Journal of immunology 160, 3305-3314.

Pao, L.I., Lam, K.P., Henderson, J.M., Kutok, J.L., Alimzhanov, M., Nitschke, L., Thomas, M.L., Neel, B.G., and Rajewsky, K. (2007). B cell-specific deletion of protein-tyrosine phosphatase Shp1 promotes B-1a cell development and causes systemic autoimmunity. Immunity 27, 35-48.

Pasqualucci, L., Compagno, M., Houldsworth, J., Monti, S., Grunn, A., Nandula, S.V., Aster, J.C., Murty, V.V., Shipp, M.A., and Dalla-Favera, R. (2006). Inactivation of the PRDM1/BLIMP1 gene in diffuse large $B$ cell lymphoma. The Journal of experimental medicine 203, 311-317.

Paul, J., Soujon, M., Wengner, A.M., Zitzmann-Kolbe, S., Sturz, A., Haike, K., Keng Magdalene, K.H., Tan, S.H., Lange, M., Tan, S.Y., Mumberg, D., Lim, S.T., Ziegelbauer, K., and Liu, N. (2017). Simultaneous Inhibition of PI3Kdelta and PI3Kalpha Induces ABCDLBCL Regression by Blocking BCR-Dependent and -Independent Activation of NFkappaB and AKT. Cancer cell 31, 64-78.

Pfeifer, M., Grau, M., Lenze, D., Wenzel, S.S., Wolf, A., Wollert-Wulf, B., Dietze, K., Nogai, H., Storek, B., Madle, H., Dorken, B., Janz, M., Dirnhofer, S., Lenz, P., Hummel, M., Tzankov, A., and Lenz, G. (2013). PTEN loss defines a PI3K/AKT pathway-dependent germinal center subtype of diffuse large B-cell lymphoma. Proceedings of the National Academy of Sciences of the United States of America 110, 12420-12425.

Pfeifer, M., and Lenz, G. (2013). PI3K/AKT addiction in subsets of diffuse large B-cell lymphoma. Cell Cycle 12, 3347-3348.

Pirkl, M., Hand, E., Kube, D., and Spang, R. (2016). Analyzing synergistic and nonsynergistic interactions in signalling pathways using Boolean Nested Effect Models. Bioinformatics 32, 893-900.

Polack, A., Hortnagel, K., Pajic, A., Christoph, B., Baier, B., Falk, M., Mautner, J., Geltinger, C., Bornkamm, G.W., and Kempkes, B. (1996). c-myc activation renders proliferation of Epstein-Barr virus (EBV)-transformed cells independent of EBV nuclear antigen 2 and latent membrane protein 1. Proceedings of the National Academy of Sciences of the United States of America 93, 10411-10416.

Pone, E.J., Zan, H., Zhang, J., Al-Qahtani, A., Xu, Z., and Casali, P. (2010). Toll-like receptors and $B$-cell receptors synergize to induce immunoglobulin class-switch DNA recombination: relevance to microbial antibody responses. Critical reviews in immunology 30, 1-29.

Rawlings, J.S., Rosler, K.M., and Harrison, D.A. (2004). The JAK/STAT signaling pathway. J Cell Sci 117, 1281-1283.

Reth, M., and Brummer, T. (2004). Feedback regulation of lymphocyte signalling. Nature reviews Immunology 4, 269-277.

Richter, J., Schlesner, M., Hoffmann, S., Kreuz, M., Leich, E., Burkhardt, B., Rosolowski, M., Ammerpohl, O., Wagener, R., Bernhart, S.H., Lenze, D., Szczepanowski, M., Paulsen, M., Lipinski, S., Russell, R.B., Adam-Klages, S., Apic, G., Claviez, A., Hasenclever, D., Hovestadt, V., Hornig, N., Korbel, J.O., Kube, D., Langenberger, D., Lawerenz, C., Lisfeld, J., Meyer, K., Picelli, S., Pischimarov, J., Radlwimmer, B., Rausch, T., Rohde, M., Schilhabel, M., Scholtysik, R., Spang, R., Trautmann, H., Zenz, T., Borkhardt, A., Drexler, 
H.G., Moller, P., MacLeod, R.A., Pott, C., Schreiber, S., Trumper, L., Loeffler, M., Stadler, P.F., Lichter, P., Eils, R., Kuppers, R., Hummel, M., Klapper, W., Rosenstiel, P., Rosenwald, A., Brors, B., and Siebert, R. (2012). Recurrent mutation of the ID3 gene in Burkitt lymphoma identified by integrated genome, exome and transcriptome sequencing. Nat Genet 44, 1316-1320.

Rickert, R.C. (2013). New insights into pre-BCR and BCR signalling with relevance to $B$ cell malignancies. Nature reviews Immunology 13, 578-591.

Roskoski, R., Jr. (2012). ERK1/2 MAP kinases: structure, function, and regulation. Pharmacological research 66, 105-143.

Ruland, J., and Mak, T.W. (2003). Transducing signals from antigen receptors to nuclear factor kappaB. Immunological reviews 193, 93-100.

Sabio, G., and Davis, R.J. (2014). TNF and MAP kinase signalling pathways. Semin Immunol 26, 237-245.

Sander, S., Calado, D.P., Srinivasan, L., Kochert, K., Zhang, B., Rosolowski, M., Rodig, S.J., Holzmann, K., Stilgenbauer, S., Siebert, R., Bullinger, L., and Rajewsky, K. (2012). Synergy between PI3K signaling and MYC in Burkitt lymphomagenesis. Cancer cell 22, 167-179.

Satpathy, S., Wagner, S.A., Beli, P., Gupta, R., Kristiansen, T.A., Malinova, D., Francavilla, C., Tolar, P., Bishop, G.A., Hostager, B.S., and Choudhary, C. (2015). Systems-wide analysis of BCR signalosomes and downstream phosphorylation and ubiquitylation. Molecular systems biology 11, 1-22.

Schmitz, R., Ceribelli, M., Pittaluga, S., Wright, G., and Staudt, L.M. (2014). Oncogenic mechanisms in Burkitt lymphoma. Cold Spring Harbor perspectives in medicine 4, 1-13.

Schmitz, R., Young, R.M., Ceribelli, M., Jhavar, S., Xiao, W., Zhang, M., Wright, G., Shaffer, A.L., Hodson, D.J., Buras, E., Liu, X., Powell, J., Yang, Y., Xu, W., Zhao, H., Kohlhammer, H., Rosenwald, A., Kluin, P., Muller-Hermelink, H.K., Ott, G., Gascoyne, R.D., Connors, J.M., Rimsza, L.M., Campo, E., Jaffe, E.S., Delabie, J., Smeland, E.B., Ogwang, M.D., Reynolds, S.J., Fisher, R.I., Braziel, R.M., Tubbs, R.R., Cook, J.R., Weisenburger, D.D., Chan, W.C., Pittaluga, S., Wilson, W., Waldmann, T.A., Rowe, M., Mbulaiteye, S.M., Rickinson, A.B., and Staudt, L.M. (2012). Burkitt lymphoma pathogenesis and therapeutic targets from structural and functional genomics. Nature 490, 116-120.

Schneeberger, C., Speiser, P., Kury, F., and Zeillinger, R. (1995). Quantitative detection of reverse transcriptase-PCR products by means of a novel and sensitive DNA stain. PCR methods and applications 4, 234-238.

Schreiber, E., Matthias, P., Muller, M.M., and Schaffner, W. (1989). Rapid detection of octamer binding proteins with 'mini-extracts', prepared from a small number of cells. Nucleic acids research 17, 6419.

Shaul, Y.D., and Seger, R. (2007). The MEK/ERK cascade: from signaling specificity to diverse functions. Biochimica et biophysica acta 1773, 1213-1226.

Shinohara, H., Yasuda, T., Aiba, Y., Sanjo, H., Hamadate, M., Watarai, H., Sakurai, H., and Kurosaki, T. (2005). PKC beta regulates BCR-mediated IKK activation by facilitating the interaction between TAK1 and CARMA1. The Journal of experimental medicine 202, 1423-1431. 
Smith, S.M. (2015). New drugs for the treatment of non-Hodgkin lymphomas. Chinese clinical oncology 4, 1-11.

Spender, L.C., and Inman, G.J. (2012). Phosphoinositide 3-kinase/AKT/mTORC1/2 signaling determines sensitivity of Burkitt's lymphoma cells to $\mathrm{BH} 3$ mimetics. Molecular cancer research : MCR 10, 347-359.

Spender, L.C., and Inman, G.J. (2014). Developments in Burkitt's lymphoma: novel cooperations in oncogenic MYC signaling. Cancer management and research 6, 27-38.

Srinivasan, L., Sasaki, Y., Calado, D.P., Zhang, B., Paik, J.H., DePinho, R.A., Kutok, J.L., Kearney, J.F., Otipoby, K.L., and Rajewsky, K. (2009). PI3 kinase signals BCR-dependent mature B cell survival. Cell 139, 573-586.

Staudt, L.M. (2010). Oncogenic activation of NF-kappaB. Cold Spring Harbor perspectives in biology 2, 1-30.

Steelman, L.S., Franklin, R.A., Abrams, S.L., Chappell, W., Kempf, C.R., Basecke, J., Stivala, F., Donia, M., Fagone, P., Nicoletti, F., Libra, M., Ruvolo, P., Ruvolo, V., Evangelisti, C., Martelli, A.M., and McCubrey, J.A. (2011). Roles of the Ras/Raf/MEK/ERK pathway in leukemia therapy. Leukemia 25, 1080-1094.

Stein, H., and Hummel, M. (2006). Histopathology in the light of molecular profiling. Annals of oncology : official journal of the European Society for Medical Oncology 17 Suppl 4, iv5-iv7.

Sun, H., Charles, C.H., Lau, L.F., and Tonks, N.K. (1993). MKP-1 (3CH134), an immediate early gene product, is a dual specificity phosphatase that dephosphorylates MAP kinase in vivo. Cell 75, 487-493.

Supek, F., Bosnjak, M., Skunca, N., and Smuc, T. (2011). REVIGO summarizes and visualizes long lists of gene ontology terms. PloS one 6, 1-9.

Szklarczyk, D., Franceschini, A., Wyder, S., Forslund, K., Heller, D., Huerta-Cepas, J., Simonovic, M., Roth, A., Santos, A., Tsafou, K.P., Kuhn, M., Bork, P., Jensen, L.J., and von Mering, C. (2015). STRING v10: protein-protein interaction networks, integrated over the tree of life. Nucleic acids research 43, D447-452.

Takata, M., and Kurosaki, T. (1996). A role for Bruton's tyrosine kinase in B cell antigen receptor-mediated activation of phospholipase C-gamma 2. The Journal of experimental medicine 184, 31-40.

Tartaglia, M., Martinelli, S., Cazzaniga, G., Cordeddu, V., lavarone, I., Spinelli, M., Palmi, C., Carta, C., Pession, A., Arico, M., Masera, G., Basso, G., Sorcini, M., Gelb, B.D., and Biondi, A. (2004). Genetic evidence for lineage-related and differentiation stage-related contribution of somatic PTPN11 mutations to leukemogenesis in childhood acute leukemia. Blood 104, 307-313.

Thome, M., Charton, J.E., Pelzer, C., and Hailfinger, S. (2010). Antigen receptor signaling to NF-kappaB via CARMA1, BCL10, and MALT1. Cold Spring Harbor perspectives in biology 2, 1-16

Towbin, H., Staehelin, T., and Gordon, J. (1979). Electrophoretic transfer of proteins from polyacrylamide gels to nitrocellulose sheets: procedure and some applications. 
Proceedings of the National Academy of Sciences of the United States of America 76, 4350-4354.

Tremblay, F., Brule, S., Hee Um, S., Li, Y., Masuda, K., Roden, M., Sun, X.J., Krebs, M., Polakiewicz, R.D., Thomas, G., and Marette, A. (2007). Identification of IRS-1 Ser-1101 as a target of S6K1 in nutrient- and obesity-induced insulin resistance. Proceedings of the National Academy of Sciences of the United States of America 104, 14056-14061.

Trempolec, N., Dave-Coll, N., and Nebreda, A.R. (2013a). SnapShot: p38 MAPK signaling. Cell 152, 656-656 e651.

Trempolec, N., Dave-Coll, N., and Nebreda, A.R. (2013b). SnapShot: p38 MAPK substrates. Cell 152, 924-924 e921.

Tweeddale, M.E., Lim, B., Jamal, N., Robinson, J., Zalcberg, J., Lockwood, G., Minden, M.D., and Messner, H.A. (1987). The presence of clonogenic cells in high-grade malignant lymphoma: a prognostic factor. Blood 69, 1307-1314.

Vainchenker, W., and Constantinescu, S.N. (2013). JAK/STAT signaling in hematological malignancies. Oncogene 32, 2601-2613.

Vardiman, J.W., Thiele, J., Arber, D.A., Brunning, R.D., Borowitz, M.J., Porwit, A., Harris, N.L., Le Beau, M.M., Hellstrom-Lindberg, E., Tefferi, A., and Bloomfield, C.D. (2009). The 2008 revision of the World Health Organization (WHO) classification of myeloid neoplasms and acute leukemia: rationale and important changes. Blood 114, 937-951.

Vega, G.G., Aviles-Salas, A., Chalapud, J.R., Martinez-Paniagua, M., Pelayo, R., Mayani, H., Hernandez-Pando, R., Martinez-Maza, O., Huerta-Yepez, S., Bonavida, B., and Vega, M.I. (2015). P38 MAPK expression and activation predicts failure of response to CHOP in patients with Diffuse Large B-Cell Lymphoma. BMC Cancer 15, 722 721-712.

Victora, G.D., Dominguez-Sola, D., Holmes, A.B., Deroubaix, S., Dalla-Favera, R., and Nussenzweig, M.C. (2012). Identification of human germinal center light and dark zone cells and their relationship to human B-cell lymphomas. Blood 120, 2240-2248.

Victora, G.D., and Nussenzweig, M.C. (2012). Germinal centers. Annu Rev Immunol 30, 429-457.

Victora, G.D., Schwickert, T.A., Fooksman, D.R., Kamphorst, A.O., Meyer-Hermann, M., Dustin, M.L., and Nussenzweig, M.C. (2010). Germinal center dynamics revealed by multiphoton microscopy with a photoactivatable fluorescent reporter. Cell 143, 592-605.

Von Knethen, A., Abts, H., Kube, D., Diehl, V., and Tesch, H. (1997). Expression of p56lck in B-cell neoplasias. Leukemia \& lymphoma 26, 551-562.

Wang, Z., Yang, H., Tachado, S.D., Capo-Aponte, J.E., Bildin, V.N., Koziel, H., and Reinach, P.S. (2006). Phosphatase-mediated crosstalk control of ERK and p38 MAPK signaling in corneal epithelial cells. Investigative ophthalmology \& visual science 47, 52675275.

Wartmann, M., Hofer, P., Turowski, P., Saltiel, A.R., and Hynes, N.E. (1997). Negative modulation of membrane localization of the Raf-1 protein kinase by hyperphosphorylation. The Journal of biological chemistry 272, 3915-3923.

Wellbrock, C., Karasarides, M., and Marais, R. (2004). The RAF proteins take centre stage. Nature reviews Molecular cell biology 5, 875-885. 
Wienands, J., Schweikert, J., Wollscheid, B., Jumaa, H., Nielsen, P.J., and Reth, M. (1998). SLP-65: a new signaling component in B lymphocytes which requires expression of the antigen receptor for phosphorylation. The Journal of experimental medicine 188, 791-795.

Wilson, W.H., Young, R.M., Schmitz, R., Yang, Y., Pittaluga, S., Wright, G., Lih, C.J., Williams, P.M., Shaffer, A.L., Gerecitano, J., de Vos, S., Goy, A., Kenkre, V.P., Barr, P.M., Blum, K.A., Shustov, A., Advani, R., Fowler, N.H., Vose, J.M., Elstrom, R.L., Habermann, T.M., Barrientos, J.C., McGreivy, J., Fardis, M., Chang, B.Y., Clow, F., Munneke, B., Moussa, D., Beaupre, D.M., and Staudt, L.M. (2015). Targeting B cell receptor signaling with ibrutinib in diffuse large B cell lymphoma. Nature medicine 21, 922-926.

Winslow, M.M., Gallo, E.M., Neilson, J.R., and Crabtree, G.R. (2006). The calcineurin phosphatase complex modulates immunogenic B cell responses. Immunity 24, 141-152.

Wojciechowski, W., Li, H., Marshall, S., Dell'Agnola, C., and Espinoza-Delgado, I. (2005). Enhanced expression of CD20 in human tumor B cells is controlled through ERKdependent mechanisms. Journal of immunology 174, 7859-7868.

Wolff, A., Bayerlova, M., Gaedcke, J., Kube, D., and Beissbarth, T. (2018). A comparative study of RNA-Seq and microarray data analysis on the two examples of rectal-cancer patients and Burkitt Lymphoma cells. PloS one 13, 1-16.

Yasuda, T., Kometani, K., Takahashi, N., Imai, Y., Aiba, Y., and Kurosaki, T. (2011). ERKs induce expression of the transcriptional repressor Blimp-1 and subsequent plasma cell differentiation. Science signaling 4, ra25 21-10.

Yasuda, T., Sanjo, H., Pages, G., Kawano, Y., Karasuyama, H., Pouyssegur, J., Ogata, M., and Kurosaki, T. (2008). Erk kinases link pre-B cell receptor signaling to transcriptional events required for early B cell expansion. Immunity $28,499-508$.

Yea, S.S., and Fruman, D.A. (2011). Cell signaling. New mTOR targets Grb attention. Science 332, 1270-1271.

Young, R.M., and Staudt, L.M. (2013). Targeting pathological B cell receptor signalling in lymphoid malignancies. Nature reviews Drug discovery 12, 229-243.

Young, R.M., Wu, T., Schmitz, R., Dawood, M., Xiao, W., Phelan, J.D., Xu, W., Menard, L., Meffre, E., Chan, W.C., Jaffe, E.S., Gascoyne, R.D., Campo, E., Rosenwald, A., Ott, G., Delabie, J., Rimsza, L.M., and Staudt, L.M. (2015). Survival of human lymphoma cells requires B-cell receptor engagement by self-antigens. Proceedings of the National Academy of Sciences of the United States of America 112, 13447-13454.

Zakaria, N., Mohd Yusoff, N., Zakaria, Z., Widera, D., and Yahaya, B.H. (2018). Inhibition of NF-kappaB Signaling Reduces the Stemness Characteristics of Lung Cancer Stem Cells. Front Oncol 8, 166 161-112.

Zarubin, T., and Han, J. (2005). Activation and signaling of the p38 MAP kinase pathway. Cell research 15, 11-18.

Zhang, J.X., Zhuang, W.J., Poon, K.H., Yang, M., and Fong, W.F. (2003). Induction of HL60 cell differentiation by the p38 mitogen-activated protein kinase inhibitor SB203580 is mediated through the extracellular signal-regulated kinase signaling pathway. Anti-Cancer Drugs 14, 31-38. 
Zhou, J., Zhang, Q., Henriquez, J.E., Crawford, R.B., and Kaminski, N.E. (2018). Lymphocyte-Specific Protein Tyrosine Kinase (LCK) is Involved in the Aryl Hydrocarbon Receptor-Mediated Impairment of Immunoglobulin Secretion in Human Primary B Cells. Toxicological sciences : an official journal of the Society of Toxicology 165, 322-334.

Zimmermann, S., and Moelling, K. (1999). Phosphorylation and regulation of Raf by Akt (protein kinase B). Science 286, 1741-1744.

Zmajkovicova, K., Jesenberger, V., Catalanotti, F., Baumgartner, C., Reyes, G., and Baccarini, M. (2013). MEK1 is required for PTEN membrane recruitment, AKT regulation, and the maintenance of peripheral tolerance. Mol Cell 50, 43-55. 


\section{Appendix}

Figure A-1 displays the mean $\log _{2}$ fold changes and the standard deviation of the measured protein phosphorylations after distinct pathway perturbation in BL-2 cells. The analysis was done with the multiplex immunoassay, the means were calculated with three independent experiments and the values were normalized to the untreated control. These results were evaluated by Bertram Klinger (Institute of Pathology, Charité - University Medicine Berlin) and are part of Figure 4 and Figure 6.

A

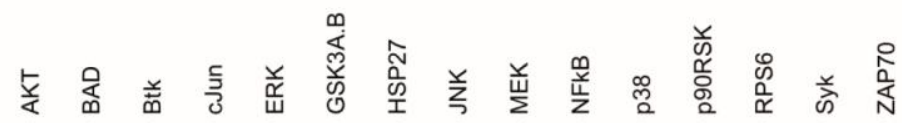

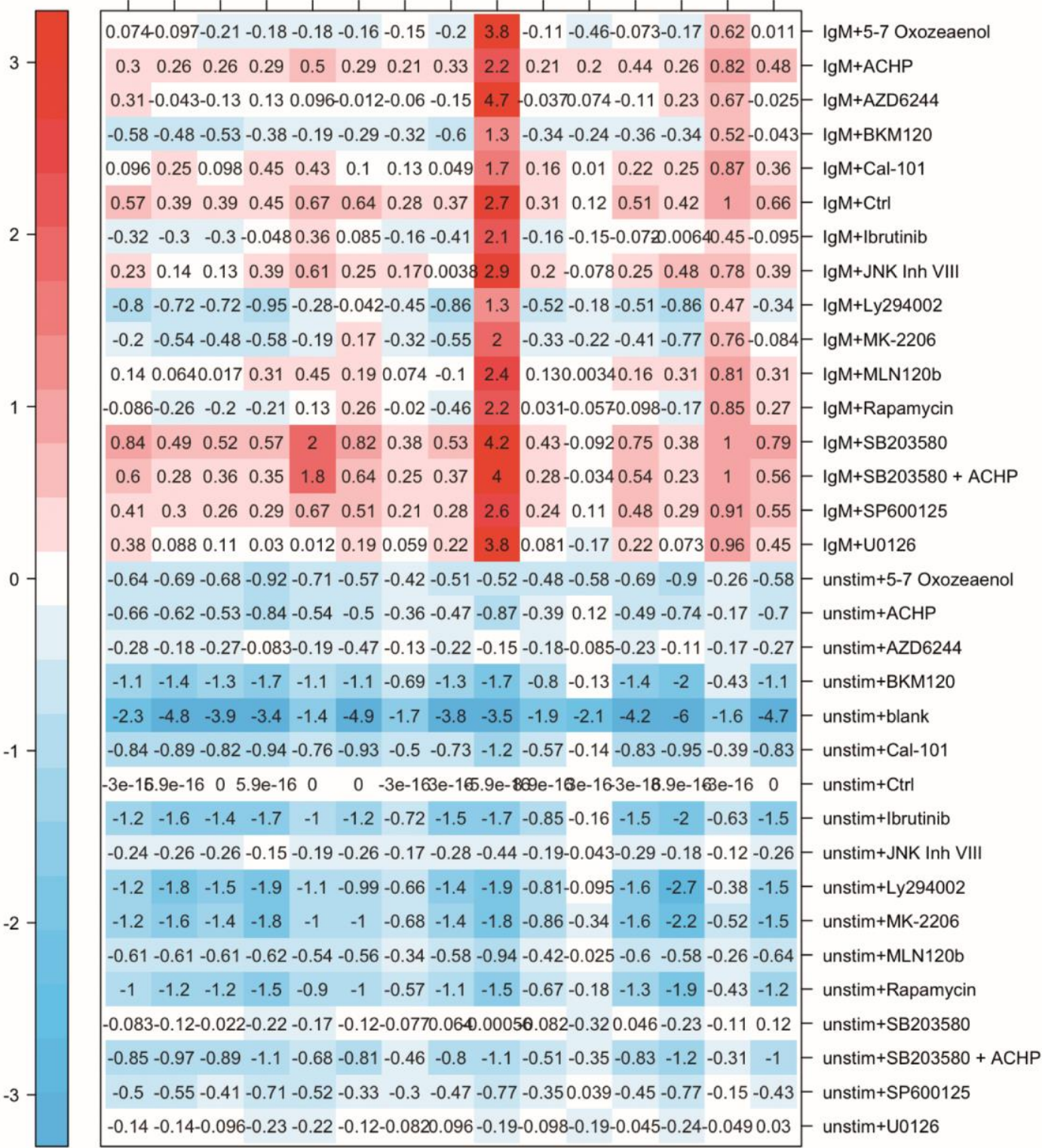




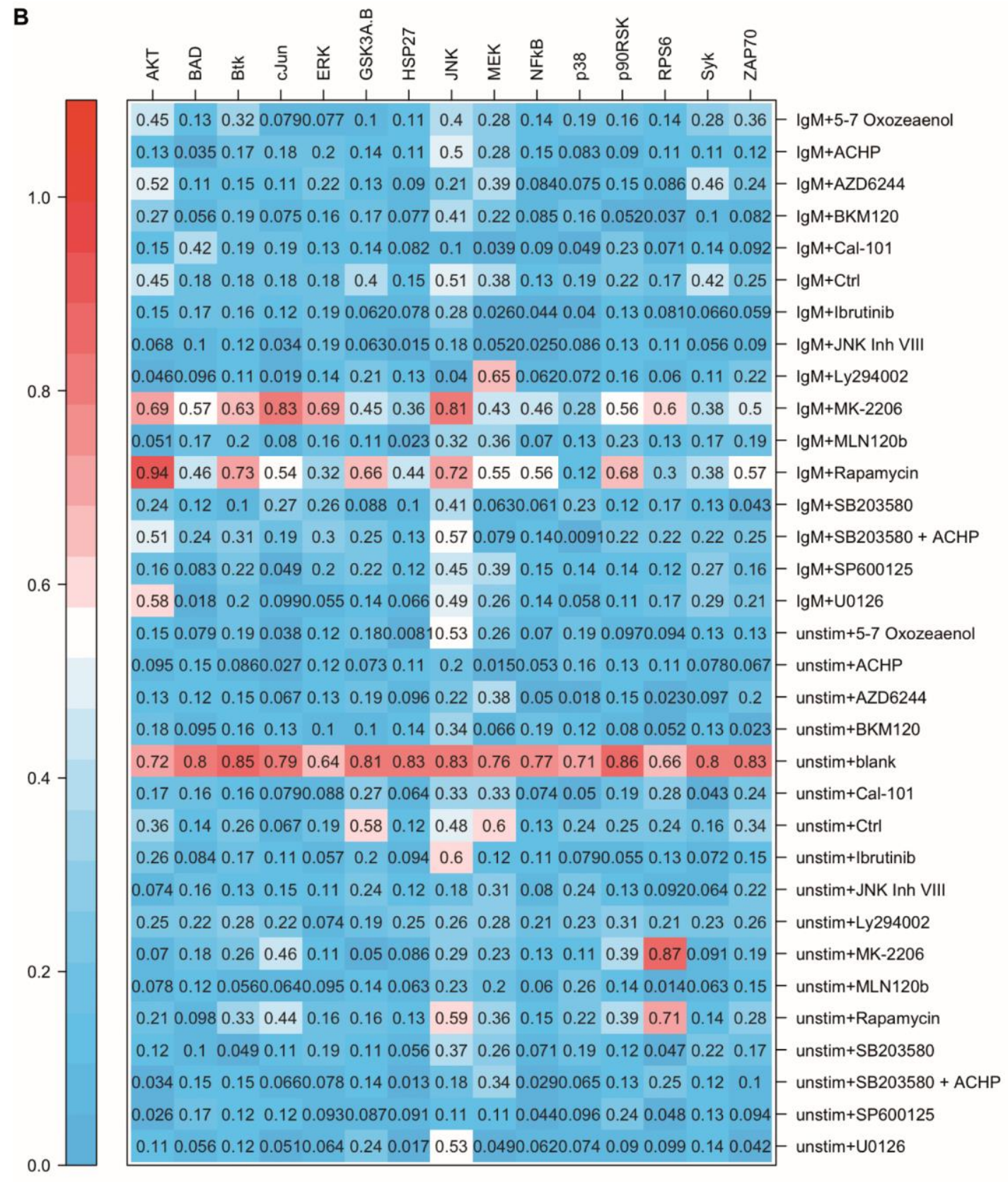

Figure A-1: Multiplex immunoassay values and standard deviation of the BL-2 cell analysis BL-2 cells were analyzed with the multiplex immunoassay after 3 hours inhibitor treatment and additionally BCR activation for 30 minutes. (A) Mean log2 fold changes of measured protein phosphorylations were presented for each pathway perturbation normalized to the untreated control $(n=3)$. (B) Standard deviation of A was calculated. This evaluation was done by Bertram Klinger (Institute of Pathology, Charité - University Medicine Berlin). 
Figure A-2 displays the mean $\log _{2}$ fold changes and the standard deviation of the measured protein phosphorylations after distinct pathway perturbation in BL-41 cells. The analysis was done with the multiplex immunoassay, the means were calculated with three independent experiments and the values were normalized to the untreated control. These results were evaluated by Bertram Klinger (Institute of Pathology, Charité - University Medicine Berlin) and are part of Figure 4 and Figure 6

A

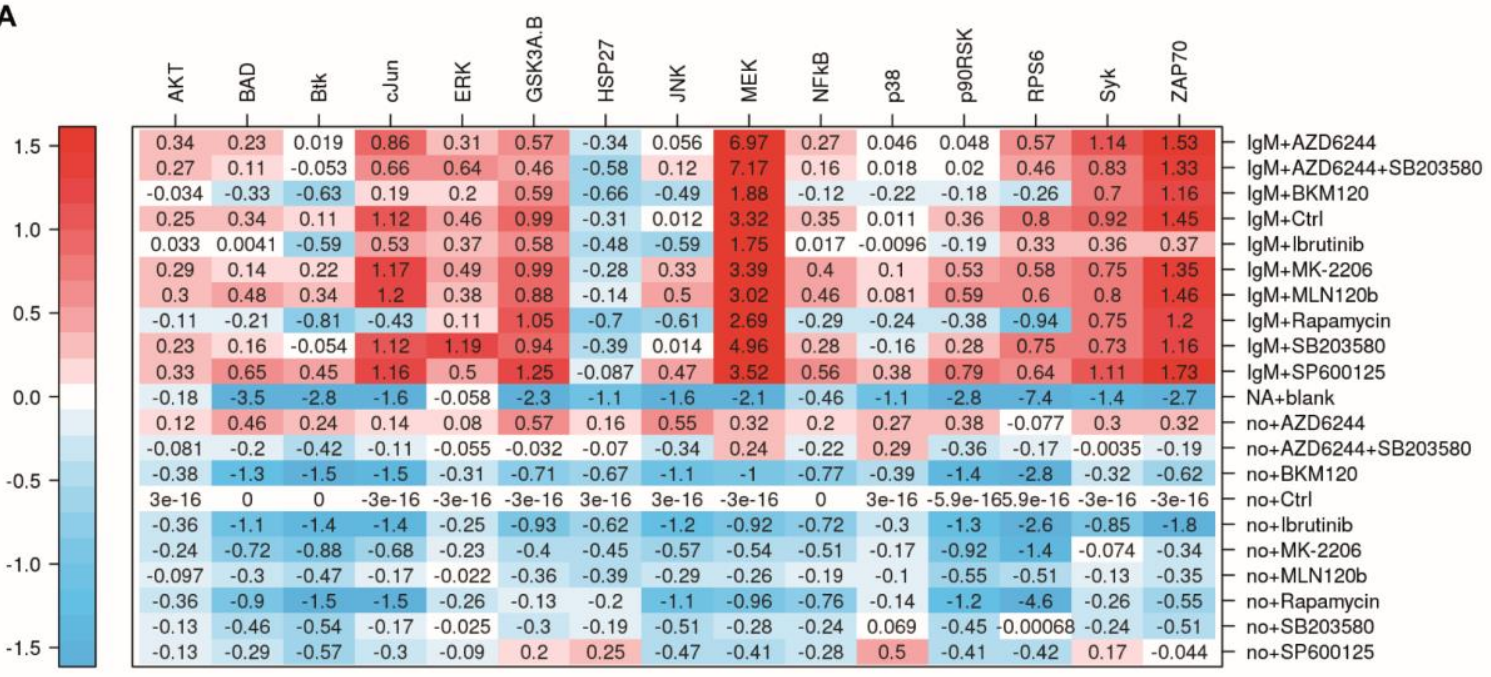

B

\begin{tabular}{|c|c|c|c|c|c|c|c|c|c|c|c|c|c|c|c|}
\hline $\begin{array}{l}\frac{5}{<} \\
1\end{array}$ & 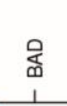 & $\begin{array}{l}\text { 盖 } \\
\end{array}$ & $\begin{array}{l}5 \\
\text { J } \\
0 \\
1\end{array}$ & 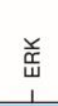 & 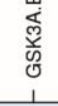 & $\begin{array}{l}\hat{N} \\
0 \\
\frac{0}{1} \\
1 \\
1\end{array}$ & $\begin{array}{l}\frac{2}{2} \\
1 \\
1\end{array}$ & 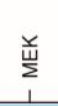 & $\begin{array}{l}\frac{9}{\frac{y}{z}} \\
\frac{1}{z} \\
\end{array}$ & $\begin{array}{l}\infty \\
\varrho \\
\varrho\end{array}$ & 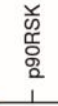 & $\begin{array}{l}\infty \\
\frac{0}{\pi} \\
\text { 1 } \\
1\end{array}$ & 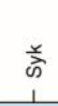 & $\begin{array}{c}0 \\
\frac{0}{2} \\
\frac{1}{N} \\
1 \\
1\end{array}$ & \\
\hline 0.15 & 0.49 & 0.49 & 0.27 & 0.038 & 0.43 & 0.13 & 0.39 & 0.013 & 0.19 & 0.13 & 0.53 & 0.23 & 0.28 & 0.46 & - IgM+AZD6244 \\
\hline 0.018 & 0.076 & 0.18 & 0.3 & 0.053 & 0.63 & 0.049 & 0.13 & 0.079 & 0.06 & 0.19 & 0.28 & 0.11 & 0.17 & 0.33 & - $\lg M+A Z D 6244+S B 20358$ \\
\hline 0.13 & 0.48 & 0.56 & 0.26 & 0.1 & 0.71 & 0.15 & 0.48 & 0.3 & 0.34 & 0.18 & 0.57 & 0.17 & 0.23 & 0.34 & - $\lg M+B K M 120$ \\
\hline 0.028 & 0.22 & 0.27 & 0.17 & 0.12 & 0.29 & 0.036 & 0.26 & 0.2 & 0.037 & 0.046 & 0.33 & 0.069 & 0.12 & 0.22 & $-\lg M+C \operatorname{ctl} \mid$ \\
\hline 0.12 & 0.24 & 0.41 & 0.24 & 0.072 & 0.67 & 0.14 & 0.36 & 0.42 & 0.12 & 0.19 & 0.43 & 0.15 & 0.32 & 0.45 & - lgM+lbrutinib \\
\hline 0.12 & 0.52 & 0.36 & 0.35 & 0.13 & 0.21 & 0.11 & 0.31 & 0.086 & 0.096 & 0.077 & 0.35 & 0.12 & 0.082 & 0.17 & - IgM+MK-2206 \\
\hline 0.14 & 0.75 & 0.86 & 0.1 & 0.036 & 0.61 & 0.15 & 0.88 & 0.41 & 0.25 & 0.12 & 0.84 & 0.077 & 0.29 & 0.49 & - IgM+MLN120b \\
\hline 0.078 & 0.13 & 0.18 & 0.17 & 0.058 & 0.27 & 0.054 & 0.25 & 0.43 & 0.066 & 0.046 & 0.17 & 0.17 & 0.11 & 0.17 & - IgM+Rapamycin \\
\hline 0.037 & 0.23 & 0.39 & 0.35 & 0.82 & 0.46 & 0.076 & 0.18 & 1.44 & 0.087 & 0.12 & 0.28 & 0.043 & 0.28 & 0.4 & - $\lg M+S B 203580$ \\
\hline 0.055 & 0.66 & 0.62 & 0.38 & 0.013 & 0.87 & 0.16 & 0.91 & 0.23 & 0.12 & 0.057 & 0.65 & 0.12 & 0.45 & 0.53 & - $\lg M+S P 600125$ \\
\hline 0.11 & 0.51 & 0.51 & 0.27 & 0.079 & 0.75 & 0.23 & 1.04 & 0.45 & 0.27 & 0.23 & 0.5 & 0.073 & 0.58 & 0.96 & - no+AZD6244 \\
\hline 0.075 & 0.1 & 0.086 & 0.31 & 0.098 & 0.32 & 0.37 & 0.094 & 0.071 & 0.1 & 0.26 & 0.15 & 0.057 & 0.17 & 0.23 & - no+AZD6244+SB203580 \\
\hline 0.056 & 0.17 & 0.13 & 0.081 & 0.059 & 0.008 & 0.05 & 0.18 & 0.048 & 0.036 & 0.13 & 0.05 & 0.066 & 0.43 & 0.42 & - no+BKM120 \\
\hline 0.041 & 0.059 & 0.11 & 0.13 & 0.05 & 0.12 & 0.074 & 0.15 & 0.07 & 0.089 & 0.055 & 0.09 & 0.093 & 0.22 & 0.2 & - no+Ctrl \\
\hline 0.11 & 0.35 & 0.33 & 0.18 & 0.037 & 0.4 & 0.2 & 0.28 & 0.31 & 0.14 & 0.26 & 0.3 & 0.63 & 0.43 & 0.49 & - no+lbrutinib \\
\hline 0.09 & 0.18 & 0.091 & 0.27 & 0.13 & 0.21 & 0.071 & 0.16 & 0.12 & 0.046 & 0.1 & 0.095 & 0.25 & 0.35 & 0.4 & - no+MK-2206 \\
\hline 0.091 & 0.13 & 0.35 & 0.24 & 0.09 & 0.23 & 0.14 & 0.34 & 0.3 & 0.11 & 0.048 & 0.27 & 0.079 & 0.41 & 0.6 & - no+MLN120b \\
\hline 0.056 & 0.14 & 0.16 & 0.044 & 0.022 & 0.15 & 0.23 & 0.21 & 0.11 & 0.054 & 0.13 & 0.1 & 0.047 & 0.5 & 0.46 & - no+Rapamycin \\
\hline 0.031 & 0.0064 & 0.14 & 0.32 & 0.14 & 0.14 & 0.081 & 0.088 & 0.17 & 0.15 & 0.12 & 0.11 & 0.1 & 0.41 & 0.28 & - no+SB203580 \\
\hline 0.088 & 0.3 & 0.32 & 0.35 & 0.15 & 0.25 & 0.39 & 0.32 & 0.28 & 0.12 & 0.088 & 0.2 & 0.05 & 0.47 & 0.53 & - no+SP600125 \\
\hline
\end{tabular}

Figure A-2: Multiplex immunoassay values and standard deviation of the BL-41 cell analysis BL-41 cells were analyzed with the multiplex immunoassay after 3 hours inhibitor treatment and additionally BCR activation for 30 minutes. (A) Mean $\log _{2}$ fold changes of measured protein phosphorylations were presented for each pathway perturbation normalized to the untreated control $(n=3)$. (B) Standard deviation of A was calculated. This evaluation was done by Bertram Klinger (Institute of Pathology, Charité - University Medicine Berlin). 
Figure A-3 presents the starting network of the BCR signaling which was complemented with the results of the multiplex immunoassay to generate a refined network model. This scheme was created by Bertram Klinger (Institute of Pathology, Charité - University Medicine Berlin).

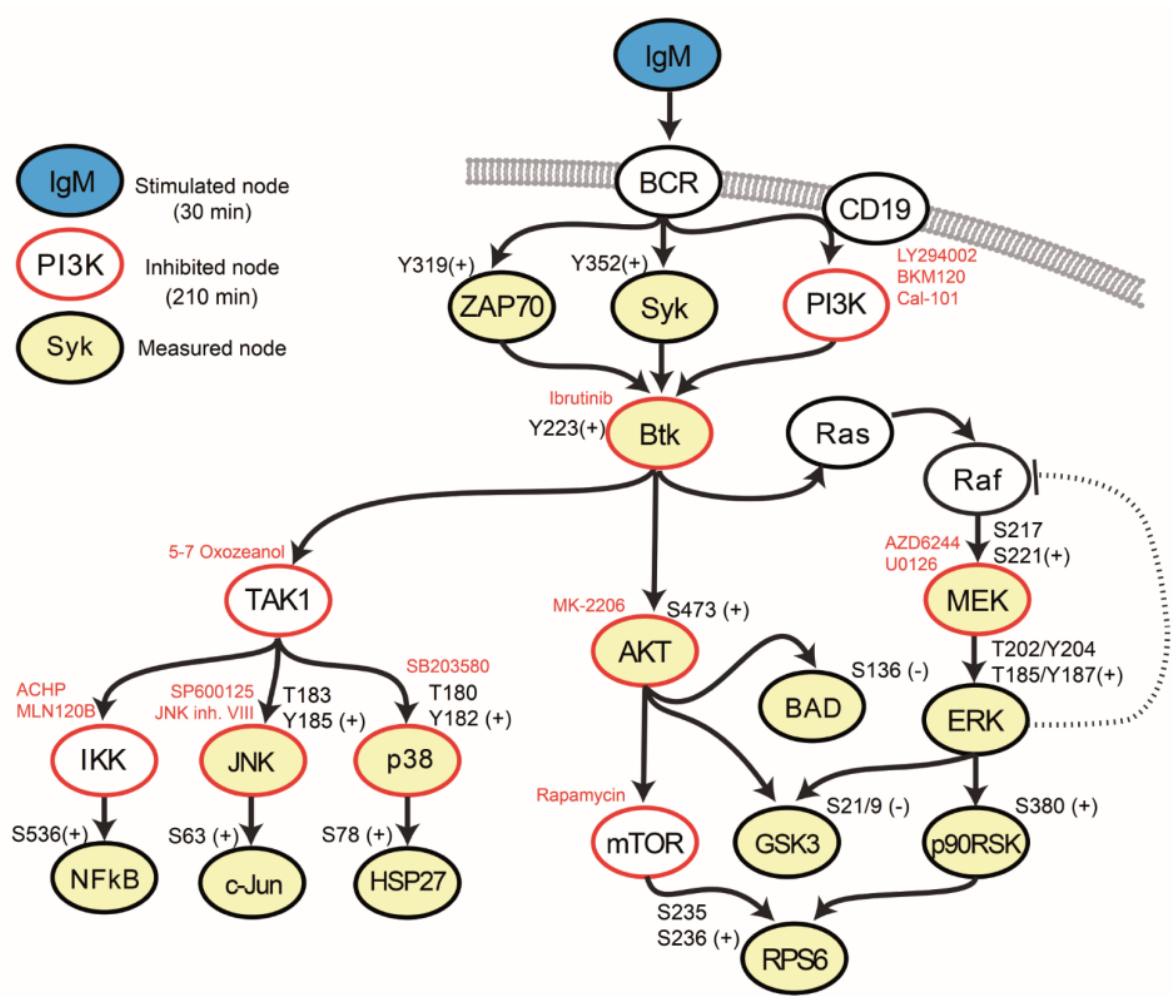

Figure A-3: Literature-derived network model with the experimental setup

Network model scheme based on the review of Hendriks et al. was created by Bertram Klinger (Institute of Pathology, Charité - University Medicine Berlin) (Hendriks et al., 2014). Stimulated (blue), inhibited (red) and measured (yellow) nodes are displayed. The corresponding details present the used inhibitors (red) and the measured protein phosphorylations (black). 
Figure A-4 displays the generated network model of BL-41 cells which was done by Bertram Klinger (Institute of Pathology, Charité - University Medicine Berlin) and belongs to Figure 7.

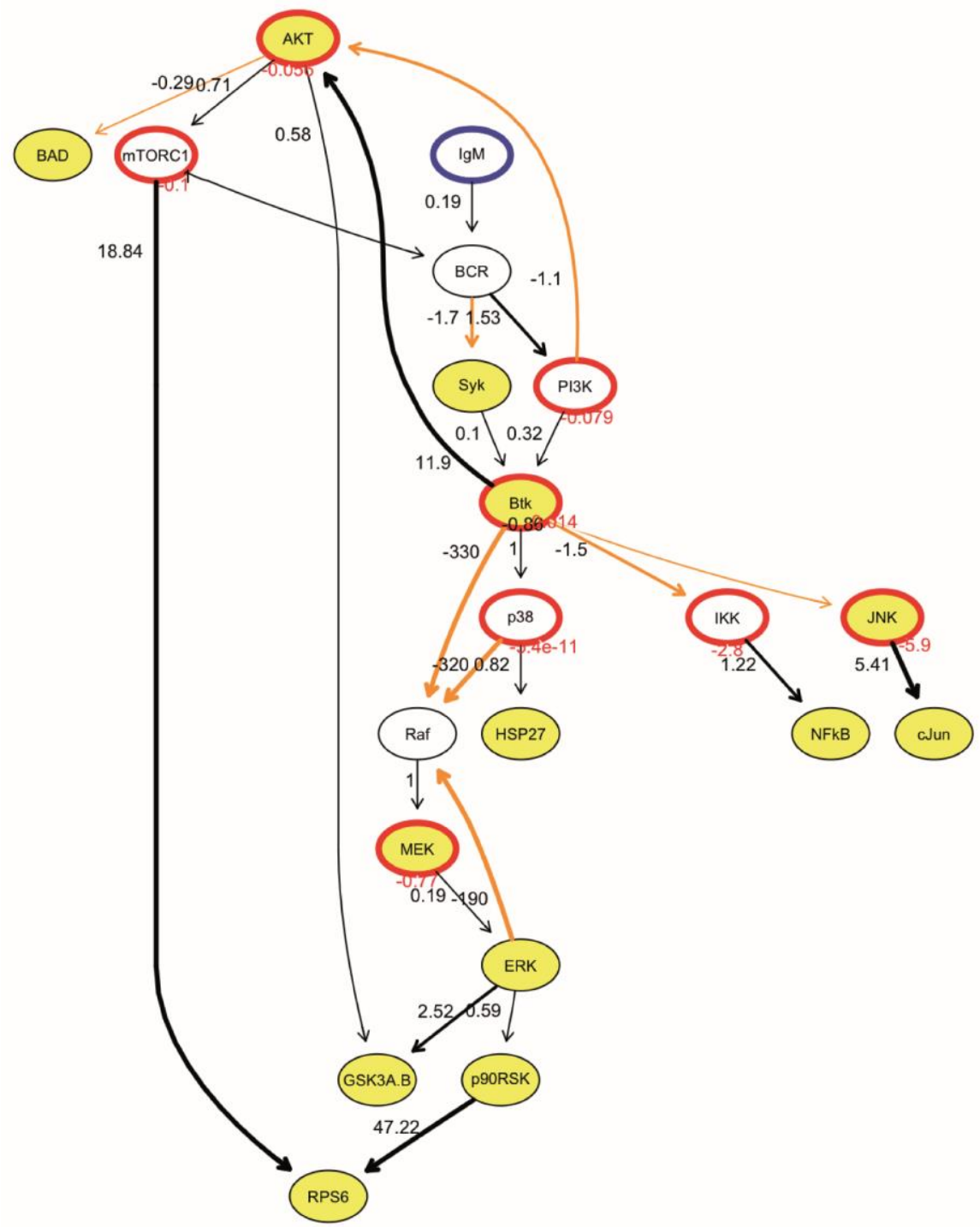

Figure A-4: Network model of BCR signaling interactions and feedbacks of BL-41 cells Network model of BL-41 cells based on Modular Response Analysis by STASTNet was calculated by Bertram Klinger (Institute of Pathology, Charité - University Medicine Berlin). Stimulated (blue), inhibited (red) and measured (yellow) nodes are displayed. The corresponding numbers indicate the inhibition strength (red) and the value of the local response coefficients (black). Arrows present interaction with positive (black) or negative (orange) local response coefficients. 
Figure A-5 displays the mean $\log _{2}$ fold changes and the standard deviation of the measured protein phosphorylations after distinct pathway perturbation in $\mathrm{HBL}-1$ and OCI-LY3 cells. The analysis was done with the multiplex immunoassay, the means were calculated with three independent experiments and the values were normalized to the untreated control. These results were evaluated by Bertram Klinger (Institute of Pathology, Charité - University Medicine Berlin) and are part of Figure 15.

A

\begin{tabular}{|c|c|c|c|c|c|c|c|c|c|c|c|c|c|c|c|}
\hline $\begin{array}{l}\frac{5}{<} \\
\mathbb{4} \\
1\end{array}$ & $\begin{array}{l}\text { Q } \\
\text { ๓ } \\
\text { । }\end{array}$ & $\begin{array}{l}\text { 弟 } \\
1\end{array}$ & $\begin{array}{l}5 \\
5 \\
0 \\
1\end{array}$ & 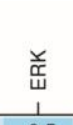 & 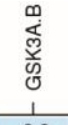 & $\begin{array}{l}\hat{N} \\
\text { ज़ } \\
\frac{1}{1} \\
1\end{array}$ & $\begin{array}{l}\text { 并 } \\
1 \\
\end{array}$ & $\begin{array}{l}\stackrel{x}{W} \\
\perp\end{array}$ & 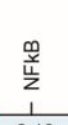 & $\begin{array}{l}\infty \\
\stackrel{\aleph}{\alpha} \\
\end{array}$ & 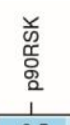 & 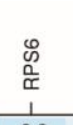 & $\begin{array}{l}\text { 离 } \\
1 \\
1\end{array}$ & 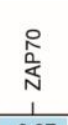 & \\
\hline-0.13 & -0.26 & -0.4 & -0.3 & -0.5 & -0.3 & -0.17 & -0.25 & 1.35 & -0.19 & -0.17 & -0.5 & -0.3 & -0.12 & -0.37 & - HBL-1+AZD6244 \\
\hline-0.23 & -0.39 & -0.67 & -0.44 & -0.58 & -0.38 & -0.37 & -0.41 & 2.51 & -0.45 & -0.27 & -0.68 & -0.51 & -0.064 & -0.49 & - HBL-1+AZD6244+SB203580 \\
\hline-0.43 & $3-0.95$ & -1.1 & -1.7 & -0.48 & -0.59 & -0.73 & -0.9 & -0.48 & -0.97 & -0.53 & -0.99 & -2.7 & 0.039 & -0.9 & - HBL-1+BKM120 \\
\hline 0 & $5.9 e-16$ & $3 e-16$ & $-5.9 e-16$ & $6-3 e-16$ & $-3 e-16$ & 0 & $3 e-16$ & 0 & 0 & $3 e-16$ & $-3 e-16-$ & $5.9 e-16$ & 0 & 0 & - HBL-1+Ctrl \\
\hline-0.41 & \begin{tabular}{l|l}
1 & -1.2 \\
\end{tabular} & -1.3 & -1.3 & -0.75 & -1.1 & -0.65 & -1 & -1.9 & -0.88 & -0.42 & -1.2 & -1.7 & -0.43 & -1.2 & - HBL-1+lbrutinib \\
\hline 0.11 & 0.26 & 0.24 & 0.15 & 0.25 & 0.21 & 0.021 & 0.27 & 1.39 & 0.058 & 0.036 & 0.27 & -0.19 & 0.22 & 0.19 & - HBL-1+MK-2206 \\
\hline-0.12 & -0.038 & -0.36 & -0.2 & 0.0067 & 0.0081 & -0.28 & -0.11 & 1.14 & -0.26 & -0.096 & -0.21 & -0.41 & -0.1 & -0.36 & - HBL-1+MLN120b \\
\hline-0.55 & -0.99 & -1.3 & -2.3 & -0.41 & -0.51 & -0.82 & -1.1 & -0.3 & -1.1 & -0.55 & -1.1 & -4 & -0.34 & -1.2 & - HBL-1+Rapamycin \\
\hline-0.027 & $27-0.067$ & -0.28 & 0.12 & 1.1 & 0.094 & -0.041 & -0.075 & 2.99 & -0.064 & -0.09 & -0.026 & 0.074 & -0.19 & -0.25 & - HBL-1+SB203580 \\
\hline-0.25 & $\begin{array}{ll}5 & -0.76 \\
\end{array}$ & -0.72 & -0.32 & -0.17 & -0.49 & -0.34 & -0.44 & -0.32 & -0.4 & -0.21 & -0.58 & -0.47 & -0.3 & -0.73 & - HBL-1+SP600125 \\
\hline 0.0031 & $31-0.00014$ & -0.11 & -0.067 & -0.23 & -0.12 & 0.043 & 0.038 & 2.47 & 0.024 & 0.21 & -0.14 & -0.37 & 0.1 & -0.019 & - OCI-LY3+AZD6244 \\
\hline-0.089 & $39-0.051$ & -0.37 & -0.42 & -0.32 & -0.3 & -0.24 & 0.088 & 3.78 & -0.31 & -0.53 & -0.59 & -1.4 & -0.067 & -0.2 & - OCI-LY3+AZD6244+SB203580 \\
\hline-0.23 & $\begin{array}{ll}3 & -0.72 \\
\end{array}$ & -0.63 & -0.95 & -0.28 & -0.17 & -0.45 & -0.32 & -0.26 & -0.55 & -0.11 & -0.42 & -2.4 & -0.23 & -0.36 & - OCI-LY3+BKM120 \\
\hline 0 & $-5.9 \mathrm{e}-16$ & 0 & 0 & 0 & $-3 e-16$ & $3 e-16$ & $-3 e-16$ & 0 & $3 e-16$ & 0 & $-3 e-16$ & 0 & $3 e-16$ & $-3 e-16$ & $-\mathrm{OCl}-\mathrm{LY} 3+\mathrm{Ctrl}$ \\
\hline-0.27 & $\begin{array}{ll}7 & -0.69\end{array}$ & -0.77 & -1.7 & -0.25 & -0.57 & -0.34 & -0.82 & -0.33 & -0.36 & -0.33 & -0.63 & -1.3 & -0.023 & -0.48 & - OCI-LY3+lbrutinib \\
\hline-0.076 & $6-0.41$ & -0.027 & -0.55 & -0.14 & 0.39 & -0.3 & 0.16 & 0.14 & -0.31 & 0.07 & -0.046 & -1.1 & -0.023 & 0.036 & - OCI-LY3+MK-2206 \\
\hline-0.15 & -0.18 & -0.51 & -0.65 & -0.35 & -0.28 & -0.41 & -0.076 & -0.32 & -0.54 & -0.16 & -0.47 & -2 & -0.24 & -0.36 & - OCI-LY3+MLN120b \\
\hline-0.19 & -0.25 & -0.68 & -1.2 & -0.3 & -0.29 & -0.23 & -0.52 & -0.54 & -0.33 & -0.1 & -0.48 & -3 & -0.15 & -0.41 & - OCI-LY3+Rapamycin \\
\hline-0.055 & $\begin{array}{cc}5 & -0.07\end{array}$ & -0.17 & -0.064 & -0.071 & 0.11 & -0.13 & 0.01 & 0.039 & -0.16 & -0.55 & -0.15 & -0.5 & -0.086 & -0.12 & - OCI-LY3+SB203580 \\
\hline-0.088 & -0.3 & -0.44 & -0.8 & -0.15 & -0.073 & -0.22 & -0.29 & -0.12 & -0.37 & 0.069 & -0.3 & -1.4 & -0.092 & -0.25 & - OCI-LY3+SP600125 \\
\hline
\end{tabular}

B

\begin{tabular}{|c|c|c|c|c|c|c|c|c|c|c|c|c|c|c|c|}
\hline$\frac{5}{<}$ & ๕̊ & 盖 & క్ & $\begin{array}{l}\stackrel{\text { ్ }}{山} \\
\text { }\end{array}$ & 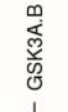 & $\begin{array}{l}\text { No } \\
\text { ô } \\
\text { ox }\end{array}$ & 首 & 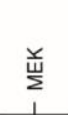 & $\frac{9}{\frac{9}{u}}$ & ळ్ & 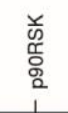 & $\begin{array}{l}\text { ஜ } \\
\stackrel{0}{\alpha}\end{array}$ & के & 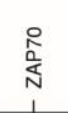 & \\
\hline 0.031 & 0.094 & 0.073 & 0.29 & 0.12 & 0.21 & 0.18 & 0.049 & 0.57 & 0.12 & 0.11 & 0.033 & 0.17 & 0.27 & 0.14 & HBL-1+AZD6244 \\
\hline 0.02 & 0.08 & 0.19 & 0.26 & 0.12 & 0.22 & 0.14 & 0.047 & 0.51 & 0.17 & 0.13 & 0.044 & 0.24 & 0.4 & 0.18 & - HBL-1+AZD6244+SB203580 \\
\hline 0.14 & 0.43 & 0.15 & 0.16 & 0.093 & 0.25 & 0.16 & 0.17 & 0.38 & 0.21 & 0.13 & 0.11 & 0.11 & 0.41 & 0.26 & - HBL-1+BKM120 \\
\hline 0.081 & 0.11 & 0.1 & 0.39 & 0.099 & 0.19 & 0.079 & 0.03 & 0.41 & 0.12 & 0.095 & 0.14 & 0.18 & 0.16 & 0.13 & - HBL-1+Ctrl \\
\hline 0.099 & 0.33 & 0.38 & 0.21 & 0.16 & 0.47 & 0.17 & 0.14 & 0.3 & 0.19 & 0.099 & 0.25 & 0.2 & 0.41 & 0.33 & - HBL-1+lbrutinib \\
\hline 0.12 & 0.59 & 0.39 & 0.27 & 0.22 & 0.28 & 0.13 & 0.32 & 0.89 & 0.16 & 0.056 & 0.4 & 0.26 & 0.32 & 0.32 & - HBL-1+MK-2206 \\
\hline 0.047 & 0.058 & 0.34 & 0.28 & 0.019 & 0.28 & 0.04 & 0.24 & 0.39 & 0.046 & 0.07 & 0.19 & 0.31 & 0.23 & 0.21 & - HBL-1+MLN120b \\
\hline 0.054 & 0.12 & 0.12 & 0.067 & 0.086 & 0.28 & 0.13 & 0.13 & 0.7 & 0.14 & 0.17 & 0.046 & 0.12 & 0.21 & 0.14 & - HBL-1+Rapamycin \\
\hline 0.047 & 0.12 & 0.19 & 0.38 & 0.27 & 0.21 & 0.04 & 0.18 & 0.59 & 0.054 & 0.048 & 0.11 & 0.34 & 0.26 & 0.23 & - HBL-1+SB203580 \\
\hline 0.028 & 0.2 & 0.34 & 0.39 & 0.16 & 0.18 & 0.11 & 0.17 & 0.53 & 0.078 & 0.089 & 0.22 & 0.35 & 0.16 & 0.16 & - HBL-1+SP600125 \\
\hline 0.032 & 0.16 & 0.033 & 0.057 & 0.047 & 0.11 & 0.075 & 0.086 & 0.11 & 0.026 & 0.13 & 0.1 & 0.31 & 0.023 & 0.086 & - OCl-LY3+AZD6244 \\
\hline 0.083 & 0.17 & 0.18 & 0.11 & 0.051 & 0.13 & 0.063 & 0.14 & 0.12 & 0.06 & 0.091 & 0.098 & 0.17 & 0.062 & 0.14 & - OCI-LY3+AZD6244+SB20358C \\
\hline 0.042 & 0.07 & 0.12 & 0.18 & 0.056 & 0.19 & 0.088 & 0.15 & 0.015 & 0.02 & 0.097 & 0.054 & 0.16 & 0.067 & 0.069 & - OCI-LY3+BKM120 \\
\hline 0.022 & 0.081 & 0.066 & 0.18 & 0.02 & 0.058 & 0.022 & 0.065 & 0.096 & 0.037 & 0.11 & 0.032 & 0.2 & 0.077 & 0.093 & $-\mathrm{OCl}-\mathrm{LY} 3+\mathrm{Ctrl}$ \\
\hline 0.074 & 0.12 & 0.19 & 0.65 & 0.19 & 0.26 & 0.23 & 0.25 & 0.14 & 0.15 & 0.26 & 0.17 & 2.35 & 0.085 & 0.051 & - OCI-LY3+lbrutinib \\
\hline 0.025 & 0.13 & 0.52 & 0.29 & 0.045 & 0.37 & 0.052 & 0.58 & 0.36 & 0.016 & 0.073 & 0.35 & 0.44 & 0.096 & 0.36 & - OCI-LY3+MK-2206 \\
\hline 0.09 & 0.04 & 0.26 & 0.077 & 0.057 & 0.1 & 0.14 & 0.091 & 0.13 & 0.17 & 0.11 & 0.18 & 0.11 & 0.082 & 0.09 & - OCI-LY3+MLN120b \\
\hline 0.074 & 0.22 & 0.17 & 0.17 & 0.1 & 0.09 & 0.17 & 0.11 & 0.08 & 0.11 & 0.1 & 0.081 & 0.18 & 0.19 & 0.17 & - OCl-LY3+Rapamycin \\
\hline 0.065 & 0.044 & 0.19 & 0.039 & 0 & 0.24 & 0.095 & 0.086 & 0.095 & 0.13 & 0.043 & 0.15 & 0.12 & 0.027 & 0.044 & - OCI-LY3+SB203580 \\
\hline 0.058 & 0.24 & 0.11 & 0.2 & 0.093 & 0.14 & 0.072 & 0.24 & 0.14 & 0.088 & 0.096 & 0.053 & 0.26 & 0.039 & 0.076 & - OCI-LY3+SP600125 \\
\hline
\end{tabular}

Figure A-5: Multiplex immunoassay values and standard deviation of ABC DLBCLs analyses HBL-1 and OCl-LY3 cells were analyzed with the multiplex immunoassay after 3 hours inhibitor treatment. (A) Mean $\log _{2}$ fold changes of measured protein phosphorylations were presented for each pathway perturbation normalized to the untreated control $(n=3)$. (B) Standard deviation of A was calculated. This evaluation was done by Bertram Klinger (Institute of Pathology, Charité University Medicine Berlin). 
A list of all quantified protein phosphorylations in P493-6 cells, which are shown in Figure 19 , is attached. This includes the name of protein, phosphorylation site, mean $\log _{2}$ values of the light/heavy ratio, $p$-values and the measured intensities. The calculation of the $\log _{2}$ values were done by Jasmin Corso from the Bioanalytical Mass Spectrometry Group of Henning Urlaub (Max-Planck-Institute for Biophysical Chemistry, Goettingen).

The significantly changed phosphosites after IL10+CpG stimulation displayed in Figure 19 are listed in Table A-1. This includes the name of protein, phosphorylation site, mean $\log _{2}$ values of the light/heavy ratio, $p$-values and the measured intensities.

Table A-1: The changed phosphosites after IL10+CpG stimulation

\begin{tabular}{|c|c|c|c|c|c|c|c|}
\hline $\mathrm{T}$ : Gene names & $\begin{array}{c}\text { C: } \\
\text { Amino } \\
\text { acid }\end{array}$ & $\begin{array}{c}\text { N: } \\
\text { Position }\end{array}$ & $\begin{array}{l}\text { light Myc } \\
\text { low /heavy } \\
\text { Myc high } \\
\text { IL10+CpG }\end{array}$ & $\begin{array}{c}\text { light Myc } \\
\text { low } \\
\text { IL10+CpG } \\
\text { /heavy } \\
\text { Myc high } \\
\text { IL10+CpG }\end{array}$ & $\begin{array}{c}\text { Differ- } \\
\text { ence } \\
\text { IL10+ } \\
\text { CpG } \\
\text { - Ctrl }\end{array}$ & $\begin{array}{l}\text { Individual } \\
\text { p-value }\end{array}$ & N: Intensity \\
\hline $\begin{array}{l}\text { ABL2 } \\
\text { ACIN1;DKFZp667N107 }\end{array}$ & $\begin{array}{l}\mathrm{S} \\
\mathrm{S}\end{array}$ & $\begin{array}{l}936 \\
652\end{array}$ & $\begin{array}{l}-0,90 \\
-0,81\end{array}$ & $\begin{array}{l}-0,07 \\
-0,18\end{array}$ & $\begin{array}{l}0,83 \\
0,62\end{array}$ & $\begin{array}{l}0,0011 \\
0,0142\end{array}$ & $\begin{array}{l}103040000 \\
399670000\end{array}$ \\
\hline ADD1 & $\mathrm{T}$ & 11 & 0,09 & 1,13 & 1,05 & 0,0001 & 1904600000 \\
\hline ADRBK1 & $S$ & 652 & $-1,07$ & $-0,11$ & 0,96 & 0,0002 & 574690000 \\
\hline AHNAK & $S$ & 3426 & $-1,56$ & 1,35 & 2,91 & 0,0001 & 461270000 \\
\hline AHNAK & $S$ & 5110 & 0,32 & 1,26 & 0,94 & 0,0002 & 642210000 \\
\hline AHNAK & S & 135 & 1,19 & 2,07 & 0,88 & 0,0005 & 3396400000 \\
\hline AHNAK & $S$ & 210 & 0,63 & 1,27 & 0,64 & 0,0118 & 962530000 \\
\hline AHNAK & $\mathrm{S}$ & 5830 & 0,87 & 1,30 & $-0,73$ & 0,0187 & 50311000 \\
\hline AKAP1 & $\mathrm{S}$ & 150 & $-2,06$ & $-1,32$ & 0,74 & 0,0035 & 101850000 \\
\hline AKAP13 & $S$ & 2709 & $-1,77$ & 0,74 & 2,34 & 0,0001 & 78983000 \\
\hline AMFR;hCG_1811773 & $S$ & 191 & $-0,91$ & 0,03 & 0,94 & 0,0002 & 61806000 \\
\hline ANKRD28 & $S$ & 1011 & $-1,27$ & $-0,29$ & 0,70 & 0,0241 & 30248000 \\
\hline APOB48R;APOBR & $\mathrm{T}$ & 563 & 1,29 & 1,96 & 0,67 & 0,0313 & 67005000 \\
\hline APPL1 & $S$ & 401 & 1,22 & 0,62 & $-0,64$ & 0,0147 & 185830000 \\
\hline ARHGAP30 & $\mathrm{S}$ & 240 & $-0,54$ & 0,15 & 0,69 & 0,0067 & 67400000 \\
\hline ARHGAP4 & $S$ & 340 & $-0,75$ & 0,22 & 0,96 & 0,0001 & 291210000 \\
\hline ARHGEF6 & $S$ & 684 & 1,26 & 0,86 & $-0,69$ & 0,0089 & 103420000 \\
\hline ASAP1 & $S$ & 660 & 0,79 & $-0,15$ & $-1,06$ & 0,0001 & 54716000 \\
\hline BET1;DKFZp781C0425 & S & 50 & $-0,13$ & 0,44 & 0,75 & 0,0157 & 131550000 \\
\hline BIN1 & $\mathrm{S}$ & 267 & 0,86 & 0,22 & $-0,64$ & 0,0031 & 650660000 \\
\hline C2orf49 & S & 151 & $-0,93$ & 0,24 & 1,17 & 0,0001 & 187440000 \\
\hline CAMSAP1 & $S$ & 945 & $-1,00$ & $-0,06$ & 0,94 & 0,0002 & 212820000 \\
\hline CARHSP1 & S & 30 & 0,28 & $-0,46$ & $-0,74$ & 0,0001 & 1537300000 \\
\hline CCDC55;NSRP1 & $\mathrm{T}$ & 221 & $-0,19$ & $-0,82$ & $-0,80$ & 0,0025 & 117450000 \\
\hline CD2AP & $S$ & 458 & 2,01 & 1,14 & $-0,87$ & 0,0001 & 597740000 \\
\hline CD72 & $Y$ & 7 & 3,44 & 2,74 & $-0,70$ & 0,0011 & 44524000 \\
\hline CDC42EP3 & $S$ & 144 & 1,59 & 2,33 & 0,74 & 0,0178 & 95180000 \\
\hline
\end{tabular}




\begin{tabular}{|c|c|c|c|c|c|c|c|}
\hline T: Gene names & $\begin{array}{c}\text { C: } \\
\text { Amino } \\
\text { acid }\end{array}$ & $\begin{array}{c}\mathrm{N}: \\
\text { Position }\end{array}$ & $\begin{array}{c}\text { light Myc } \\
\text { low /heavy } \\
\text { Myc high } \\
\text { IL10+CpG }\end{array}$ & $\begin{array}{c}\text { light Myc } \\
\text { low } \\
\text { IL10+CpG } \\
\text { /heavy } \\
\text { Myc high } \\
\text { IL10+CpG }\end{array}$ & $\begin{array}{l}\text { Differ- } \\
\text { ence } \\
\text { IL10+ } \\
\text { CpG } \\
\text { - Ctrl }\end{array}$ & $\begin{array}{c}\text { Individual } \\
\text { p-value }\end{array}$ & N: Intensity \\
\hline CDV3 & $\bar{Y}$ & 190 & $-0,11$ & 0,85 & 0,81 & 0,0094 & 530910000 \\
\hline CENPF & $S$ & 3119 & $-1,62$ & $-2,35$ & $-0,72$ & 0,0201 & 26095000 \\
\hline CHAMP1 & $S$ & 427 & $-0,69$ & 0,10 & 0,78 & 0,0020 & 824110000 \\
\hline $\mathrm{CIC} ; \mathrm{CIC} / \mathrm{DUX} 4$ fusion & $S$ & 1405 & $-0,17$ & 0,59 & 0,77 & 0,0138 & 34471000 \\
\hline CLASP2;DKFZp686D11262 & $S$ & 934 & $-1,53$ & $-0,44$ & 1,10 & 0,0004 & 44827000 \\
\hline CLTA & $S$ & 105 & $-0,80$ & 0,15 & 0,95 & 0,0002 & 1956200000 \\
\hline DIDO1 & S & 1456 & $-0,96$ & 0,10 & 1,05 & 0,0001 & 1413300000 \\
\hline DKC1 & $S$ & 485 & $-1,35$ & $-0,59$ & 0,76 & 0,0026 & 121250000 \\
\hline DKFZp781H1925;EIF2AK3 & $S$ & 564 & $-2,12$ & $-0,54$ & 1,32 & 0,0001 & 44186000 \\
\hline DNMBP & $S$ & 684 & $-1,40$ & 0,16 & 1,12 & 0,0003 & 17740000 \\
\hline DNMT1 & $S$ & 130 & $-2,47$ & $-0,63$ & 1,89 & 0,0001 & 136570000 \\
\hline DOCK2 & $S$ & 1685 & $-0,63$ & 0,36 & 0,99 & 0,0001 & 4787800000 \\
\hline DTL & $S$ & 236 & $-2,57$ & $-1,26$ & 1,31 & 0,0001 & 31988000 \\
\hline DYNC1LI1 & $S$ & 516 & $-1,26$ & 0,10 & 1,37 & 0,0001 & 3187700000 \\
\hline EIF4B & $S$ & 382 & $-1,23$ & $-0,69$ & 0,62 & 0,0471 & 973860000 \\
\hline ELMSAN1;C14orf43 & $S$ & 996 & $-2,32$ & $-0,72$ & 1,61 & 0,0001 & 10157000 \\
\hline ELMSAN1;C14orf43 & $S$ & 461 & $-1,05$ & 0,02 & 1,07 & 0,0001 & 1275800000 \\
\hline EPB41L1 & $\mathrm{T}$ & 378 & $-0,51$ & 0,78 & 1,29 & 0,0001 & 140210000 \\
\hline EPS15 & $S$ & 796 & $-0,98$ & 0,65 & 1,63 & 0,0001 & 227790000 \\
\hline EPS15L1 & $S$ & 376 & $-2,70$ & $-1,32$ & 1,28 & 0,0001 & 180320000 \\
\hline ESCO2 & $S$ & 512 & $-1,32$ & 0,12 & 1,45 & 0,0001 & 97003000 \\
\hline FAM195A & $S$ & 82 & $-1,83$ & $-0,86$ & 0,97 & 0,0001 & 249730000 \\
\hline FAM21C;FAM21A & $S$ & 728 & $-0,35$ & 0,48 & 0,78 & 0,0123 & 141120000 \\
\hline FAM21C;FAM21A & $S$ & 288 & $-0,43$ & 0,33 & 0,75 & 0,0152 & 144640000 \\
\hline FBRSL1 & $S$ & 937 & $-2,05$ & $-2,69$ & $-0,64$ & 0,0031 & 146610000 \\
\hline $\mathrm{FCHO} 1$ & $S$ & 520 & 0,23 & 0,84 & 0,61 & 0,0171 & 153990000 \\
\hline FHOD1 & $S$ & 549 & $-1,44$ & $-0,02$ & 1,43 & 0,0001 & 107880000 \\
\hline GABPA & $S$ & 303 & $-2,99$ & 0,10 & 3,08 & 0,0001 & 100250000 \\
\hline GATAD2B & $S$ & 470 & $-1,51$ & $-0,30$ & 1,26 & 0,0001 & 212070000 \\
\hline GBF1 & $S$ & 1784 & $-1,67$ & 0,44 & 2,16 & 0,0001 & 103570000 \\
\hline GCC2 & $S$ & 554 & $-1,96$ & $-0,09$ & 1,87 & 0,0001 & 491240000 \\
\hline GCC2 & $S$ & 409 & $-1,02$ & $-0,03$ & 1,13 & 0,0003 & 47754000 \\
\hline GPATCH8 & $S$ & 1107 & $-0,63$ & 0,13 & 0,76 & 0,0027 & 609850000 \\
\hline GPS1 & $S$ & 454 & 0,48 & $-0,21$ & $-0,69$ & 0,0013 & 284620000 \\
\hline $\begin{array}{l}\text { GTF3C1;DKFZp686A111;DK } \\
\text { FZp68600870 }\end{array}$ & $S$ & 1068 & $-0,26$ & $-1,07$ & $-0,90$ & 0,0007 & 124150000 \\
\hline HAUS6 & $\mathrm{T}$ & 448 & $-3,07$ & $-0,61$ & 2,46 & 0,0001 & 324730000 \\
\hline hCG_1989366;NUP50 & $S$ & 221 & $-2,36$ & $-1,58$ & 0,79 & 0,0019 & 107330000 \\
\hline HEL-S-102;HSPB1 & $S$ & 15 & $-2,62$ & 0,42 & 3,04 & 0,0001 & 60325000 \\
\hline HEL-S-102;HSPB1 & $S$ & 82 & $-0,75$ & 0,29 & 1,03 & 0,0001 & $2,0624 \mathrm{E}+10$ \\
\hline HEL-S-270;ANXA2;ANXA2P2 & $S$ & 26 & 0,69 & $-0,34$ & $-1,03$ & 0,0001 & 114390000 \\
\hline HEL-S-37;LCP1 & $S$ & 5 & 1,38 & 2,31 & 0,94 & 0,0002 & $1,2519 \mathrm{E}+10$ \\
\hline HNRPD;HNRNPD & $S$ & 87 & $-0,55$ & 0,57 & 1,12 & 0,0003 & 347070000 \\
\hline HSH2D & $S$ & 210 & 0,61 & 1,41 & 0,80 & 0,0017 & 302560000 \\
\hline
\end{tabular}




\begin{tabular}{|c|c|c|c|c|c|c|c|}
\hline T: Gene names & $\begin{array}{c}\text { C: } \\
\text { Amino } \\
\text { acid }\end{array}$ & $\begin{array}{c}\text { N: } \\
\text { Position }\end{array}$ & $\begin{array}{l}\text { light Myc } \\
\text { low /heavy } \\
\text { Myc high } \\
\text { IL10+CpG }\end{array}$ & $\begin{array}{c}\text { light Myc } \\
\text { low } \\
\text { IL10+CpG } \\
\text { /heavy } \\
\text { Myc high } \\
\text { IL10+CpG }\end{array}$ & $\begin{array}{l}\text { Differ- } \\
\text { ence } \\
\text { IL10+ } \\
\text { CpG } \\
\text { - Ctrl }\end{array}$ & $\begin{array}{l}\text { Individual } \\
\text { p-value }\end{array}$ & N: Intensity \\
\hline INPP5D & $\mathrm{S}$ & 971 & 0,64 & 1,34 & 0,75 & 0,0155 & 2998000000 \\
\hline INPP5D & $\mathrm{T}$ & 963 & 0,64 & 1,33 & 0,75 & 0,0166 & 154000000 \\
\hline IQGAP2 & $S$ & 16 & 0,56 & 1,39 & 0,83 & 0,0012 & 348440000 \\
\hline IRF2BP2 & $S$ & 240 & $-0,70$ & 0,04 & 0,74 & 0,0036 & 201260000 \\
\hline IRF2BP2 & $\mathrm{S}$ & 457 & 0,38 & $-0,58$ & $-0,72$ & 0,0063 & 172360000 \\
\hline JUN;JUND & $S$ & 100 & $-1,13$ & 0,88 & 2,01 & 0,0001 & 112020000 \\
\hline KCNAB2 & $S$ & 9 & $-0,64$ & $-1,25$ & $-0,68$ & 0,0100 & 88158000 \\
\hline KDM3B;JMJD1B & $S$ & 798 & $-2,79$ & 0,22 & 3,05 & 0,0001 & 179340000 \\
\hline KDM4B;JMJD2B & $S$ & 324 & $-1,55$ & $-0,09$ & 1,35 & 0,0001 & 291910000 \\
\hline KIAA1267;KANSL1 & $S$ & 1081 & $-1,33$ & $-0,52$ & 0,80 & 0,0099 & 78048000 \\
\hline KIAA1737;CIPC & $S$ & 113 & $-1,73$ & $-1,02$ & 1,11 & 0,0004 & 78929000 \\
\hline KIF18B & $S$ & 94 & $-1,86$ & $-1,04$ & 0,82 & 0,0084 & 39783000 \\
\hline KIF4A & $S$ & 804 & $-1,30$ & 0,50 & 1,81 & 0,0001 & 52333000 \\
\hline KIFC1 & $S$ & 6 & 0,99 & $-0,17$ & $-1,16$ & 0,0001 & 534050000 \\
\hline KLC2 & $S$ & 610 & $-0,70$ & $-0,04$ & 0,66 & 0,0336 & 21362000 \\
\hline KMT2D & $S$ & 2274 & $-0,95$ & $-0,05$ & 0,90 & 0,0004 & 346090000 \\
\hline LCK;YES1;FYN;SRC & Y & 419 & $-0,07$ & 0,69 & 1,09 & 0,0005 & 282310000 \\
\hline LRMP & $S$ & 28 & $-1,47$ & $-0,36$ & 0,71 & 0,0228 & 33192000 \\
\hline LSM14A & $S$ & 183 & 0,21 & $-0,51$ & $-0,72$ & 0,0008 & 1415700000 \\
\hline LSP1 & $S$ & 177 & $-1,04$ & $-1,94$ & $-0,90$ & 0,0038 & 252620000 \\
\hline MAP1B;DKFZp686F1345 & $S$ & 1852 & $-0,78$ & 0,45 & 1,32 & 0,0001 & 55692000 \\
\hline MAP2K2;DKFZp686L02273 & $\mathrm{S}$ & 295 & $-0,13$ & 0,51 & 0,63 & 0,0416 & 243570000 \\
\hline MAP3K1 & $S$ & 1018 & 1,03 & 0,23 & $-1,10$ & 0,0001 & 77596000 \\
\hline MAPK14 & Y & 182 & $-1,99$ & 0,02 & 2,02 & 0,0001 & 2425900000 \\
\hline MAPRE1 & S & 155 & 0,11 & 1,24 & 0,91 & 0,0034 & 143320000 \\
\hline MARCKS & $S$ & 118 & $-0,77$ & 1,11 & 1,71 & 0,0001 & 216990000 \\
\hline MAVS & $S$ & 222 & $-1,54$ & 0,05 & 1,60 & 0,0001 & 143590000 \\
\hline МСMЗАР & $S$ & 153 & $-0,47$ & 0,30 & 0,77 & 0,0132 & 111300000 \\
\hline MCM4 & $S$ & 80 & $-1,51$ & $-2,34$ & $-0,65$ & 0,0135 & 32890000 \\
\hline MED24 & $\mathrm{S}$ & 38 & $-1,53$ & 0,14 & 1,40 & 0,0001 & 74733000 \\
\hline MEF2C & $S$ & 226 & $-1,57$ & 0,68 & 2,15 & 0,0001 & 173660000 \\
\hline MEF2C & $S$ & 220 & $-0,42$ & 0,44 & 0,86 & 0,0001 & 298990000 \\
\hline MEF2C & $S$ & 238 & $-0,18$ & 0,60 & 0,80 & 0,0099 & 36709000 \\
\hline $\begin{array}{l}\text { MEF2D variant } \\
\text { protein;MEF2D }\end{array}$ & $S$ & 126 & $-2,14$ & 0,04 & 2,30 & 0,0001 & 98449000 \\
\hline $\begin{array}{l}\text { MEF2D variant } \\
\text { protein;MEF2D }\end{array}$ & $S$ & 185 & $-1,05$ & 0,08 & 1,13 & 0,0001 & 187660000 \\
\hline MEPCE & $S$ & 152 & $-1,84$ & 0,01 & 1,86 & 0,0001 & 59393000 \\
\hline MICAL3 & $S$ & 1310 & $-2,19$ & $-0,85$ & 1,34 & 0,0001 & 66870000 \\
\hline MKI67 & $S$ & 1130 & $-0,52$ & 0,15 & 0,67 & 0,0321 & 59047000 \\
\hline MKL1;mkl1 & $S$ & 454 & 0,09 & 0,79 & 0,70 & 0,0060 & 172650000 \\
\hline MLLT4 & $S$ & 1234 & 0,62 & $-0,19$ & $-0,91$ & 0,0006 & 80188000 \\
\hline MPZL1 & Y & 237 & 0,02 & 0,67 & 0,65 & 0,0025 & 84308000 \\
\hline MS4A1 & $S$ & 36 & $-1,32$ & 0,84 & 2,15 & 0,0001 & 5718200000 \\
\hline MS4A1 & S & 35 & 0,47 & 1,65 & 1,27 & 0,0001 & 711490000 \\
\hline
\end{tabular}




\begin{tabular}{|c|c|c|c|c|c|c|c|}
\hline $\mathrm{T}:$ Gene names & $\begin{array}{c}\text { C: } \\
\text { Amino } \\
\text { acid }\end{array}$ & $\begin{array}{l}\text { N: } \\
\text { Position }\end{array}$ & $\begin{array}{l}\text { light Myc } \\
\text { low /heavy } \\
\text { Myc high } \\
\text { IL10+CpG }\end{array}$ & $\begin{array}{c}\text { light Myc } \\
\text { low } \\
\text { IL10+CpG } \\
\text { /heavy } \\
\text { Myc high } \\
\text { IL10+CpG }\end{array}$ & $\begin{array}{l}\text { Differ- } \\
\text { ence } \\
\text { IL10+ } \\
\text { CpG } \\
\text { - Ctrl }\end{array}$ & $\begin{array}{l}\text { Individual } \\
\text { p-value }\end{array}$ & N: Intensity \\
\hline MS4A1 & $\mathrm{S}$ & 36 & 0,47 & 1,65 & 1,27 & 0,0001 & 5718200000 \\
\hline MS4A1 & S & 218 & 0,37 & 1,60 & 1,09 & 0,0005 & 275800000 \\
\hline MS4A1 & $S$ & 35 & 1,46 & 0,74 & $-0,72$ & 0,0001 & 711490000 \\
\hline MST065;TOMM22 & $S$ & 15 & $-0,18$ & 0,41 & 0,60 & 0,0189 & 2241200000 \\
\hline MTSS1L & S & 455 & $-2,05$ & $-1,44$ & 0,83 & 0,0076 & 91922000 \\
\hline $\begin{array}{l}\text { MYO9B variant } \\
\text { protein;MYO9B }\end{array}$ & $\mathrm{T}$ & 1352 & $-2,21$ & 0,03 & 2,24 & 0,0001 & 145300000 \\
\hline $\begin{array}{l}\text { MYO9B variant } \\
\text { protein;MYO9B }\end{array}$ & $S$ & 1411 & $-2,48$ & $-0,41$ & 2,07 & 0,0001 & 352100000 \\
\hline $\begin{array}{l}\text { MYO9B variant } \\
\text { protein;MYO9B }\end{array}$ & S & 1273 & $-1,05$ & 0,26 & 1,32 & 0,0001 & 371830000 \\
\hline & S & 1296 & $-1,21$ & $-0,01$ & 1,21 & 0,0001 & 1558700000 \\
\hline NAP1L1 & $\mathrm{S}$ & 69 & $-0,28$ & $-0,71$ & $-0,66$ & 0,0347 & 77631000 \\
\hline NELFE;RDBP;NELF-E & $S$ & 51 & $-2,56$ & $-0,18$ & 2,23 & 0,0001 & 309490000 \\
\hline NELFE;RDBP;NELF-E & $S$ & 115 & $-1,94$ & $-0,49$ & 1,45 & 0,0001 & 2085800000 \\
\hline NELFE;RDBP;NELF-E & $S$ & 251 & $-1,23$ & $-0,62$ & 0,61 & 0,0166 & 376780000 \\
\hline NOC2L & $\mathrm{S}$ & 49 & $-0,79$ & $-0,39$ & 0,63 & 0,0434 & 148950000 \\
\hline NOL8 & $S$ & 1031 & $-0,75$ & $-0,12$ & 0,63 & 0,0429 & 67523000 \\
\hline NOLC1 & $\mathrm{T}$ & 610 & $-1,63$ & $-2,22$ & $-0,70$ & 0,0079 & 284210000 \\
\hline NOLC1 & $S$ & 397 & $-2,16$ & $-3,07$ & $-0,76$ & 0,0041 & 123650000 \\
\hline NOP2 & S & 58 & $-2,34$ & 0,35 & 2,68 & 0,0001 & 288400000 \\
\hline NUCKS1 & $\mathrm{T}$ & 179 & $-0,84$ & $-1,46$ & $-0,62$ & 0,0038 & 862350000 \\
\hline NUCKS1 & $S$ & 181 & $-0,81$ & $-1,47$ & $-0,66$ & 0,0023 & $2,325 E+10$ \\
\hline $\begin{array}{l}\text { NUMA1;NUMA1 variant } \\
\text { protein }\end{array}$ & $S$ & 1741 & $-1,10$ & $-0,17$ & 0,92 & 0,0003 & 376830000 \\
\hline NUP188 & S & 1709 & $-1,75$ & $-0,95$ & 0,82 & 0,0086 & 134880000 \\
\hline OSBPL11 & S & 181 & 0,49 & $-0,25$ & $-0,74$ & 0,0006 & 59062000 \\
\hline OSBPL3 & $S$ & 437 & $-0,93$ & 0,25 & 1,18 & 0,0001 & 137170000 \\
\hline OXR1 & $S$ & 113 & 0,63 & 1,35 & 0,79 & 0,0110 & 185140000 \\
\hline OXSR1 & $S$ & 359 & $-0,69$ & 0,35 & 1,04 & 0,0008 & 16074000 \\
\hline PAK1 & $\mathrm{T}$ & 114 & $-2,27$ & $-0,17$ & 2,05 & 0,0001 & 52953000 \\
\hline PAK2 & $S$ & 58 & $-1,96$ & 0,21 & 1,83 & 0,0001 & 15526000 \\
\hline PAPOLG & $S$ & 525 & $-2,70$ & $-0,56$ & 2,14 & 0,0001 & 73832000 \\
\hline PARN & $\mathrm{S}$ & 557 & $-1,47$ & $-0,59$ & 0,88 & 0,0005 & 150810000 \\
\hline PDS5A & $\mathrm{T}$ & 1208 & $-0,55$ & $-1,21$ & $-0,67$ & 0,0020 & 178640000 \\
\hline PFKL & $S$ & 775 & $-1,09$ & 0,22 & 1,31 & 0,0001 & 227750000 \\
\hline PI4K2A & $S$ & 462 & 0,54 & 1,13 & 0,77 & 0,0130 & 44624000 \\
\hline PLEKHM1 & S & 346 & 0,40 & 1,26 & 0,77 & 0,0130 & 30251000 \\
\hline PML & $S$ & 518 & 1,33 & 0,68 & $-0,65$ & 0,0027 & 596350000 \\
\hline PML & $S$ & 527 & 1,33 & 0,68 & $-0,65$ & 0,0027 & 1175000000 \\
\hline PML & $S$ & 527 & 1,64 & 0,92 & $-0,71$ & 0,0010 & 1175000000 \\
\hline PPFIBP2;DKFZp781K06126 & S & 252 & $-0,60$ & 0,63 & 1,37 & 0,0001 & 66492000 \\
\hline PPP1R10 & $\mathrm{S}$ & 313 & $-0,35$ & 0,34 & 0,68 & 0,0071 & 1795000000 \\
\hline PRKD2 & S & 198 & 1,24 & 1,73 & 0,86 & 0,0057 & 33320000 \\
\hline PROSER1 & $\mathrm{S}$ & 552 & $-1,61$ & $-0,84$ & 0,77 & 0,0023 & 163150000 \\
\hline PROSER2;C10orf47 & $S$ & 212 & $-2,61$ & $-1,70$ & 0,92 & 0,0003 & 67830000 \\
\hline
\end{tabular}




\begin{tabular}{|c|c|c|c|c|c|c|c|}
\hline $\mathrm{T}$ : Gene names & $\begin{array}{c}\text { C: } \\
\text { Amino } \\
\text { acid }\end{array}$ & $\begin{array}{l}\text { N: } \\
\text { Position }\end{array}$ & $\begin{array}{c}\text { light Myc } \\
\text { low /heavy } \\
\text { Myc high } \\
\text { IL10+CpG }\end{array}$ & $\begin{array}{c}\text { light Myc } \\
\text { low } \\
\text { IL10+CpG } \\
\text { /heavy } \\
\text { Myc high } \\
\text { IL10+CpG }\end{array}$ & $\begin{array}{l}\text { Differ- } \\
\text { ence } \\
\text { IL10+ } \\
\text { CpG } \\
\text { - Ctrl }\end{array}$ & $\begin{array}{l}\text { Individual } \\
\text { p-value }\end{array}$ & N: Intensity \\
\hline PSM8;PSMB8 & $\mathrm{S}$ & 39 & 1,35 & 1,97 & 0,62 & 0,0143 & 35825000 \\
\hline PSMA5 & $\mathrm{S}$ & 56 & $-0,33$ & 0,58 & 0,91 & 0,0004 & 372170000 \\
\hline PSMA5 & $S$ & 16 & $-0,65$ & $-0,02$ & 0,63 & 0,0124 & 367770000 \\
\hline PSMF1 & $S$ & 153 & $-1,11$ & $-0,41$ & 0,71 & 0,0054 & 601910000 \\
\hline RAF1;SRGAP3:RAF1 & $S$ & 609 & 0,36 & 1,36 & 0,81 & 0,0093 & 80432000 \\
\hline RALGPS2 & $S$ & 308 & 1,94 & 0,98 & $-1,28$ & 0,0001 & 330400000 \\
\hline RALGPS2 & $\mathrm{S}$ & 296 & 1,94 & 0,31 & $-1,95$ & 0,0001 & 407060000 \\
\hline RANBP3 & $S$ & 126 & $-0,25$ & 0,42 & 0,66 & 0,0089 & 115560000 \\
\hline RBM34 & $S$ & 17 & $-1,03$ & 0,16 & 1,19 & 0,0001 & 497240000 \\
\hline RBM7 & $S$ & 136 & $-2,48$ & 0,10 & 2,58 & 0,0001 & 106880000 \\
\hline RCSD1 & $S$ & 82 & $-2,76$ & 0,19 & 2,96 & 0,0001 & 808720000 \\
\hline RCSD1 & $\mathrm{S}$ & 83 & $-2,63$ & $-0,23$ & 2,40 & 0,0001 & 5518600000 \\
\hline RCSD1 & $\mathrm{S}$ & 68 & $-0,99$ & 0,47 & 1,46 & 0,0001 & 863810000 \\
\hline RCSD1 & $S$ & 284 & $-0,41$ & 0,58 & 0,99 & 0,0001 & 1637100000 \\
\hline RCSD1 & $S$ & 179 & $-0,17$ & 0,67 & 0,84 & 0,0010 & 3197800000 \\
\hline RCSD1 & $S$ & 120 & 0,97 & 0,20 & $-0,77$ & 0,0003 & 394830000 \\
\hline RHBDF2 & $S$ & 61 & $-0,79$ & $-0,14$ & 0,65 & 0,0107 & 143330000 \\
\hline $\mathrm{RIC1}$ & $\mathrm{S}$ & 909 & $-1,14$ & 0,97 & 2,11 & 0,0001 & 151090000 \\
\hline RNF214;DKFZp547C195 & $S$ & 15 & 0,02 & $-0,62$ & $-0,64$ & 0,0398 & 13980000 \\
\hline RPRD2 & $S$ & 1099 & $-1,32$ & 0,24 & 1,56 & 0,0001 & 289780000 \\
\hline RPS6 & $S$ & 240 & $-0,85$ & 0,01 & 0,74 & 0,0175 & 58425000 \\
\hline SACS & $S$ & 1779 & $-2,52$ & $-0,67$ & 1,85 & 0,0001 & 31816000 \\
\hline SACS & $S$ & 4264 & $-1,71$ & $-0,35$ & 1,35 & 0,0001 & 112540000 \\
\hline SART1 & $S$ & 448 & $-0,73$ & 0,19 & 0,92 & 0,0003 & 518090000 \\
\hline SCRIB & $S$ & 1448 & $-0,98$ & $-0,20$ & 0,78 & 0,0021 & 583260000 \\
\hline SCRIB & $S$ & 1486 & $-1,67$ & $-0,92$ & 0,74 & 0,0034 & 141830000 \\
\hline SCRIB & $S$ & 1475 & $-1,44$ & $-0,72$ & 0,72 & 0,0045 & 973960000 \\
\hline SCRIB & $S$ & 504 & $-1,03$ & $-0,36$ & 0,67 & 0,0088 & 1100700000 \\
\hline SEC16A & $S$ & 1964 & $-0,73$ & 0,15 & 0,76 & 0,0151 & 139820000 \\
\hline SETX & $S$ & 1366 & $-0,71$ & 0,69 & 1,40 & 0,0001 & 762420000 \\
\hline SLC29A2 & $\mathrm{S}$ & 252 & $-2,17$ & $-1,11$ & 0,92 & 0,0031 & 34200000 \\
\hline SLC9A1 & $S$ & 364 & 0,29 & 1,07 & 0,77 & 0,0023 & 272210000 \\
\hline SLC9A3R1 & $S$ & 290 & 0,20 & 1,17 & 0,97 & 0,0018 & 338940000 \\
\hline SNAPIN & $\mathrm{T}$ & 14 & $-1,16$ & $-0,47$ & 0,87 & 0,0054 & 90821000 \\
\hline SOS1 & $S$ & 752 & 2,08 & 0,74 & $-1,02$ & 0,0001 & 39536000 \\
\hline SPTBN1 & $S$ & 2254 & $-0,16$ & $-0,77$ & $-0,61$ & 0,0050 & 482260000 \\
\hline SRPK1 & $S$ & 311 & $-0,33$ & $-0,94$ & $-0,73$ & 0,0056 & 128830000 \\
\hline SRRM2;KIAA0324 & $S$ & 2272 & $-1,60$ & $-0,90$ & 0,71 & 0,0054 & 313600000 \\
\hline SRRM2;KIAA0324 & $\mathrm{T}$ & 2104 & 0,33 & $-0,53$ & $-0,87$ & 0,0001 & 2267000000 \\
\hline STAT1 & $\mathrm{S}$ & 727 & 1,33 & 2,12 & 0,78 & 0,0020 & 415540000 \\
\hline STAT3 & $\mathrm{Y}$ & 607 & $-4,26$ & 1,52 & 5,84 & 0,0001 & 515900000 \\
\hline STAT3 & $\mathrm{Y}$ & 704 & $-1,33$ & 1,51 & 2,85 & 0,0001 & 73240000 \\
\hline $\begin{array}{l}\text { STAU2;STAU2 variant } \\
\text { protein }\end{array}$ & $S$ & 105 & $-1,81$ & $-0,13$ & 1,69 & 0,0001 & 75642000 \\
\hline
\end{tabular}


Appendix

\begin{tabular}{|c|c|c|c|c|c|c|c|}
\hline $\mathrm{T}$ : Gene names & $\begin{array}{c}\text { C: } \\
\text { Amino } \\
\text { acid }\end{array}$ & $\begin{array}{c}\text { N: } \\
\text { Position }\end{array}$ & $\begin{array}{l}\text { light Myc } \\
\text { low /heavy } \\
\text { Myc high } \\
\text { IL10+CpG }\end{array}$ & $\begin{array}{c}\text { light Myc } \\
\text { low } \\
\text { IL10+CpG } \\
\text { /heavy } \\
\text { Myc high } \\
\text { IL10+CpG }\end{array}$ & $\begin{array}{l}\text { Differ- } \\
\text { ence } \\
\text { IL10+ } \\
\text { CpG } \\
\text { - Ctrl }\end{array}$ & $\begin{array}{l}\text { Individual } \\
\text { p-value }\end{array}$ & $\mathrm{N}$ : Intensity \\
\hline STK10 & $\mathrm{T}$ & 952 & 0,04 & 0,73 & 0,69 & 0,0062 & 650860000 \\
\hline STK24;HEL-S-95 & $S$ & 4 & $-0,54$ & 0,09 & 0,63 & 0,0131 & 699270000 \\
\hline STMN1 & S & 25 & $-1,13$ & 0,83 & 1,95 & 0,0001 & 5048800000 \\
\hline STX6 & $S$ & 2 & $-1,16$ & $-1,07$ & 0,63 & 0,0414 & 17434000 \\
\hline TAOK3 & $S$ & 442 & 0,68 & 0,12 & $-0,62$ & 0,0181 & 75975000 \\
\hline TBC1D4 & $S$ & 591 & $-0,83$ & $-1,58$ & $-0,75$ & 0,0005 & 1062200000 \\
\hline TBC1D5 & $S$ & 43 & 1,53 & 0,83 & $-0,70$ & 0,0012 & 153940000 \\
\hline TFAP4 & $S$ & 63 & $-3,17$ & $-3,81$ & $-0,63$ & 0,0418 & 47441000 \\
\hline THRAP3 & $S$ & 184 & $-0,03$ & $-0,89$ & $-0,85$ & 0,0012 & 57521000 \\
\hline TMEM201 & $S$ & 364 & $-1,16$ & $-2,79$ & $-1,63$ & 0,0001 & 17847000 \\
\hline TMF1 & $S$ & 333 & $-1,44$ & $-0,19$ & 1,25 & 0,0001 & 30240000 \\
\hline TMPO & $S$ & 424 & $-1,25$ & $-1,85$ & $-0,60$ & 0,0056 & 69924000 \\
\hline TMPO & $T$ & 160 & 1,23 & 0,59 & $-0,64$ & 0,0028 & 110670000 \\
\hline TNKS1BP1 & $S$ & 836 & $-2,05$ & $-0,74$ & 1,15 & 0,0002 & 70829000 \\
\hline TRIM24 & $S$ & 953 & $-2,13$ & $-0,18$ & 2,12 & 0,0001 & 242790000 \\
\hline TRIM28 & $S$ & 473 & $-0,89$ & $-0,14$ & 0,75 & 0,0034 & 1906700000 \\
\hline TRRAP & $S$ & 1772 & $-2,25$ & $-0,53$ & 2,02 & 0,0001 & 130780000 \\
\hline TSC22D4 & $S$ & 165 & 0,90 & 0,29 & $-0,64$ & 0,0150 & 22224000 \\
\hline UBAP1 & $S$ & 172 & $-0,32$ & 1,06 & 1,19 & 0,0001 & 58307000 \\
\hline UBE2O & $S$ & 839 & $-1,38$ & $-0,21$ & 1,10 & 0,0004 & 767920000 \\
\hline UBE4B & $S$ & 105 & $-1,11$ & $-0,23$ & 0,81 & 0,0092 & 179120000 \\
\hline UBR5 & $S$ & 1543 & $-0,97$ & $-0,10$ & 0,87 & 0,0006 & 577750000 \\
\hline ULK1 & $S$ & 450 & $-0,73$ & $-0,05$ & 0,68 & 0,0287 & 23276000 \\
\hline ULK1 & $S$ & 623 & $-0,70$ & $-0,03$ & 0,66 & 0,0089 & 48393000 \\
\hline USP1 & $S$ & 313 & $-1,29$ & $-0,82$ & 0,94 & 0,0025 & 109970000 \\
\hline USP10 & $S$ & 365 & $-0,27$ & $-0,65$ & $-0,62$ & 0,0182 & 33173000 \\
\hline VGLL4 & $S$ & 149 & $-2,10$ & $-1,38$ & 0,72 & 0,0008 & 58534000 \\
\hline WAC & $\mathrm{T}$ & 293 & $-2,11$ & $-0,43$ & 1,61 & 0,0001 & 40383000 \\
\hline WDR62;DKFZp434J046 & $S$ & 1123 & $-1,56$ & $-0,83$ & 0,68 & 0,0279 & 63753000 \\
\hline ZC3H12D;FLJ00361 & $S$ & 428 & $-1,55$ & 1,20 & 2,64 & 0,0001 & 81621000 \\
\hline ZC3H13 & $S$ & 993 & $-0,84$ & $-1,55$ & $-0,71$ & 0,0010 & 143120000 \\
\hline ZCCHC8 & $S$ & 420 & $-1,53$ & $-0,06$ & 1,56 & 0,0001 & 37632000 \\
\hline ZDHHC5 & $S$ & 398 & 0,21 & $-0,43$ & $-0,64$ & 0,0148 & 34041000 \\
\hline ZFC3H1 & $S$ & 352 & $-2,11$ & $-0,28$ & 1,83 & 0,0001 & 387690000 \\
\hline ZFC3H1 & $\mathrm{T}$ & 766 & $-1,33$ & $-0,19$ & 1,16 & 0,0002 & 67428000 \\
\hline $\begin{array}{l}\text { ZFP91- } \\
\text { CNTF;hCG_2042749;ZFP91 }\end{array}$ & S & 83 & 0,97 & $-0,08$ & $-1,04$ & 0,0001 & 241060000 \\
\hline ZNF609 & $S$ & 413 & $-0,86$ & 0,35 & 1,40 & 0,0001 & 268850000 \\
\hline$Z Y X$ & $S$ & 238 & 0,31 & 1,19 & 0,88 & 0,0005 & 262250000 \\
\hline \multirow[t]{2}{*}{ ZZEF1 } & $S$ & 1518 & $-0,78$ & 0,52 & 1,30 & 0,0001 & 186700000 \\
\hline & $S$ & 18 & $-1,41$ & 0,78 & 2,10 & 0,0001 & 61626000 \\
\hline
\end{tabular}


Table A-2 presents the GO annotation of the phosphorylated proteins which were significantly changed by IL10+CpG stimulation. Only terms with more than 10 counts and p-values below 0.05 were included.

Table A-2: GO annotation of phosphoproteins changed by IL10+CpG stimulation

\begin{tabular}{llllll}
\hline Category & Term & Count & $\%$ & P value & P $\log _{10}$ \\
\hline
\end{tabular}

Annotation Cluster 1 GOTERM_BP_FAT

Annotation Cluster 2 GOTERM_BP_FAT

GOTERM BP FAT GOTERM_BP_FAT GOTERM_BP_FAT GOTERM_BP_FAT GOTERM_BP_FAT GOTERM_BP_FAT GOTERM_BP_FAT GOTERM_BP_FAT

Annotation Cluster 3 GOTERM_BP_FAT GOTERM BP FAT GOTERM_BP_FAT GOTERM_BP_FAT GOTERM_BP_FAT GOTERM BP FAT GOTERM_BP_FAT GOTERM_BP_FAT GOTERM_BP_FAT GOTERM_BP_FAT GOTERM_BP_FAT GOTERM_BP_FAT GOTERM_BP_FAT

Annotation Cluster 4 GOTERM_BP_FAT GOTERM_BP_FAT GOTERM BP FAT GOTERM_BP_FAT GOTERM_BP_FAT GOTERM_BP_FAT GOTERM BP FAT GOTERM_BP_FAT GOTERM_BP_FAT GOTERM_BP_FAT GOTERM BP FAT

GOTERM_BP_FAT GOTERM_BP_FAT GOTERM_BP_FAT
Enrichment Score: 3,7335796159192123

GO:0010608 posttranscriptional regulation of gene expression

Enrichment Score: 3,3521003094311026

GO:0043933 macromolecular complex subunit organization

GO:0065003 macromolecular complex assembly

GO:0006461 protein complex assembly

GO:0070271 protein complex biogenesis

GO:0044085 cellular component biogenesis

GO:0071822 protein complex subunit organization

GO:0022607 cellular component assembly

GO:0043623 cellular protein complex assembly

GO:0034622 cellular macromolecular complex assembly

Enrichment Score: 3,1526962069914504

GO:0007049 cell cycle

GO:0000278 mitotic cell cycle

GO:0022402 cell cycle process

GO:1903047 mitotic cell cycle process

GO:0007067 mitotic nuclear division

GO:0000280 nuclear division

GO:0048285 organelle fission

GO:0044772 mitotic cell cycle phase transition

GO:0044770 cell cycle phase transition

GO:0007017 microtubule-based process

GO:0007059 chromosome segregation

GO:0000226 microtubule cytoskeleton organization

GO:0051301 cell division

\section{Enrichment Score: 2,9792557107087028}

GO:0044093 positive regulation of molecular function

GO:0035556 intracellular signal transduction

GO:0051336 regulation of hydrolase activity

GO:0043085 positive regulation of catalytic activity

GO:0065009 regulation of molecular function

GO:0051345 positive regulation of hydrolase activity

GO:0043087 regulation of GTPase activity

GO:0009966 regulation of signal transduction

GO:0010646 regulation of cell communication

GO:0023051 regulation of signalling

GO:0023014 signal transduction by protein

phosphorylation

GO:0043547 positive regulation of GTPase activity

GO:0006468 protein phosphorylation

GO:0007264 small GTPase mediated signal transduction

15

7,81

0,0003327

3,48

48

35

31

31

49

33

45

12

17

41

29

33

25

14

16

16

14

14

15

10

10

12

40

49

31

34

51

24

$\begin{array}{ccc}25,00 & 0,0000083 & 5,08 \\ 18,23 & 0,0000946 & 4,02 \\ 16,15 & 0,0001234 & 3,91 \\ 16,15 & 0,0001250 & 3,90 \\ 25,52 & 0,0002912 & 3,54 \\ 17,19 & 0,0003225 & 3,49 \\ 23,44 & 0,0003687 & 3,43 \\ 6,25 & 0,0328116 & 1,48 \\ 8,85 & 0,0491809 & 1,31\end{array}$

$\begin{array}{ccc}21,35 & 0,0000002 & 6,73 \\ 15,10 & 0,0000005 & 6,32 \\ 17,19 & 0,0000056 & 5,25 \\ 13,02 & 0,0000114 & 4,94 \\ 7,29 & 0,0004644 & 3,33 \\ 8,33 & 0,0008974 & 3,05 \\ 8,33 & 0,0017042 & 2,77 \\ 7,29 & 0,0022892 & 2,64 \\ 7,29 & 0,0038923 & 2,41 \\ 7,81 & 0,0042328 & 2,37 \\ 5,21 & 0,0071646 & 2,14 \\ 5,21 & 0,0308129 & 1,51 \\ 6,25 & 0,0328116 & 1,48\end{array}$

$20,83 \quad 0,0000057 \quad 5,24$

$25,52 \quad 0,0000287 \quad 4,54$

$16,15 \quad 0,0000298 \quad 4,53$

$\begin{array}{lll}17,71 & 0,0000362 & 4,44\end{array}$

$26,56 \quad 0,0000455 \quad 4,34$

$12,50 \quad 0,0000545 \quad 4,26$

$9,90 \quad 0,0002358 \quad 3,63$

$24,48 \quad 0,0002390 \quad 3,62$

$26,04 \quad 0,0003530 \quad 3,45$

$26,04 \quad 0,0005208 \quad 3,28$

$10,94 \quad 0,0005913 \quad 3,23$

$8,85 \quad 0,0007437 \quad 3,13$

$\begin{array}{lll}17,71 & 0,0008926 & 3,05\end{array}$

$\begin{array}{lll}7,81 & 0,0009821 \quad 3,01\end{array}$ 


\begin{tabular}{|c|c|c|c|c|c|c|}
\hline Category & Term & & Count & $\%$ & $P$ value & $\begin{array}{l}-\log _{10} \\
P \text { value }\end{array}$ \\
\hline GOTERM_BP_FAT & GO:0050790 & regulation of catalytic activity & 40 & 20,83 & 0,0011813 & 2,93 \\
\hline GOTERM_BP_FAT & GO:0016310 & phosphorylation & 37 & 19,27 & 0,0028837 & 2,54 \\
\hline GOTERM_BP_FAT & GO:1902531 & $\begin{array}{l}\text { regulation of intracellular signal } \\
\text { transduction }\end{array}$ & 30 & 15,63 & 0,0040778 & 2,39 \\
\hline GOTERM_BP_FAT & GO:0009967 & positive regulation of signal transduction & 26 & 13,54 & 0,0050427 & 2,30 \\
\hline GOTERM_BP_FAT & GO:0023056 & positive regulation of signalling & 27 & 14,06 & 0,0081512 & 2,09 \\
\hline GOTERM_BP_FAT & GO:0000165 & MAPK cascade & 17 & 8,85 & 0,0116933 & 1,93 \\
\hline GOTERM_BP_FAT & GO:0010647 & positive regulation of cell communication & 26 & 13,54 & 0,0141272 & 1,85 \\
\hline GOTERM_BP_FAT & GO:0048584 & $\begin{array}{l}\text { positive regulation of response to } \\
\text { stimulus }\end{array}$ & 30 & 15,63 & 0,0355079 & 1,45 \\
\hline
\end{tabular}

Annotation Cluster 5 GOTERM_BP_FAT GOTERM_BP_FAT GOTERM_BP_FAT GOTERM_BP_FAT GOTERM_BP_FAT GOTERM_BP_FAT GOTERM_BP_FAT GOTERM_BP_FAT GOTERM_BP_FAT GOTERM_BP_FAT GOTERM_BP_FAT GOTERM_BP_FAT GOTERM_BP_FAT GOTERM_BP_FAT GOTERM_BP_FAT GOTERM_BP_FAT GOTERM_BP_FAT GOTERM_BP_FAT GOTERM_BP_FAT GOTERM_BP_FAT GOTERM_BP_FAT GOTERM_BP_FAT GOTERM_BP_FAT GOTERM_BP_FAT GOTERM_BP_FAT

GOTERM_BP_FAT GOTERM_BP_FAT GOTERM_BP_FAT

Annotation Cluster 6 GOTERM_BP_FAT GOTERM_BP_FAT GOTERM_BP_FAT GOTERM_BP_FAT

Annotation Cluster 7 GOTERM_BP_FAT GOTERM_BP_FAT GOTERM_BP_FAT GOTERM_BP_FAT

\section{Enrichment Score: 2,859204278988926}

GO:0051641 cellular localization

GO:0051649 establishment of localization in cell

GO:0046907 intracellular transport

GO:0034504 protein localization to nucleus

GO:0017038 protein import

GO:0044744 protein targeting to nucleus

GO:0006606 protein import into nucleus

GO:1902593 single-organism nuclear import

GO:0051170 nuclear import

GO:1902582 single-organism intracellular transport

GO:0034613 cellular protein localization

GO:0070727 cellular macromolecule localization

GO:0008104 protein localization

GO:1902580 single-organism cellular localization

GO:0033365 protein localization to organelle

GO:0033036 macromolecule localization

GO:0051169 nuclear transport

GO:0006886 intracellular protein transport

GO:1903827 regulation of cellular protein localization

GO:0015031 protein transport

GO:0045184 establishment of protein localization

GO:0006913 nucleocytoplasmic transport

GO:0060341 regulation of cellular localization

GO:0051049 regulation of transport

GO:0072594 establishment of protein localization to organelle

GO:0032880 regulation of protein localization

GO:1903829 positive regulation of cellular protein localization

GO:0006605 protein targeting

\section{Enrichment Score: 2,6955951501894386}

GO:0007265 Ras protein signal transduction

GO:0043087 regulation of GTPase activity

GO:0043547 positive regulation of GTPase activity

GO:0007264 small GTPase mediated signal transduction

\section{Enrichment Score: 2,628977224705781}

GO:0051276 chromosome organization

GO:0051129 negative regulation of cellular component organization

GO:0010639 negative regulation of organelle organization

GO:0033044 regulation of chromosome organization

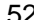

40

34

0,0001064

0,0002358

0,0007437

0,0009821

3,97

3,63

3,13

3,01

$\begin{array}{cccc}31 & 16,15 & 0,0000028 & 5,56 \\ 19 & 9,90 & 0,0000661 & 4,18 \\ 12 & 6,25 & 0,0003605 & 3,44 \\ 10 & 5,21 & 0,0027466 & 2,56\end{array}$




\begin{tabular}{|c|c|c|c|c|c|}
\hline Category & Term & Count & $\%$ & $P$ value & $\begin{array}{c}-\log _{10} \\
\text { P value }\end{array}$ \\
\hline GOTERM_BP_FAT & GO:0006325 chromatin organization & 17 & 8,85 & 0,0051095 & 2,29 \\
\hline GOTERM_BP_FAT & GO:0016570 histone modification & 12 & 6,25 & 0,0053534 & 2,27 \\
\hline GOTERM_BP_FAT & GO:0016569 covalent chromatin modification & 13 & 6,77 & 0,0104628 & 1,98 \\
\hline
\end{tabular}

Annotation Cluster 8 Enrichment Score: 2,450069107726637

GOTERM_BP_FAT GO:0006259 DNA metabolic process

GOTERM_BP_FAT

GOTERM_BP_FAT

GOTERM_BP_FAT

GO:0006281 DNA repair

GO:0006974 cellular response to DNA damage stimulus

Annotation Cluster 10 Enrichment Score: 2,2644571324985088

GOTERM_BP_FAT GO:0035556 intracellular signal transduction

GOTERM_BP_FAT GO:0033554 cellular response to stress

GOTERM_BP_FAT

GOTERM_BP_FAT

GOTERM_BP_FAT

GOTERM_BP_FAT

GOTERM_BP_FAT

GOTERM_BP_FAT

GOTERM_BP_FAT

GO:0023014 signal transduction by protein phosphorylation

GO:0006468 protein phosphorylation

GO:0016310 phosphorylation

GO:0000165 MAPK cascade

GO:0036211 protein modification process

GO:0006464 cellular protein modification process

GO:0071900 regulation of protein serine/threonine kinase activity

$\begin{array}{llll}24 & 12,50 & 0,0001842 & 3,73 \\ 13 & 6,77 & 0,0079391 & 2,10 \\ 17 & 8,85 & 0,0080847 & 2,09 \\ 10 & 5,21 & 0,0133948 & 1,87\end{array}$

$49 \quad 25,52 \quad 0,0000287 \quad 4,54$

$36 \quad 18,75 \quad 0,0000843 \quad 4,07$

$21 \quad 10,94 \quad 0,0005913 \quad 3,23$

$34 \quad 17,71 \quad 0,0008926 \quad 3,05$

$\begin{array}{llll}37 & 19,27 & 0,0028837 & 2,54\end{array}$

$17 \quad 8,85 \quad 0,0116933 \quad 1,93$

$52 \quad 27,08 \quad 0,0174549 \quad 1,76$

$52 \quad 27,08 \quad 0,0174549 \quad 1,76$

$11 \quad 5,73 \quad 0,0236050 \quad 1,63$

Annotation Cluster 11 Enrichment Score: 2,262335128933962

GOTERM_BP_FAT GO:0018193 peptidyl-amino acid modification

Annotation Cluster 12 Enrichment Score: 2,20248095655825

GOTERM_BP_FAT

GO:1902589 single-organism organelle organization

GOTERM_BP_FAT

GO:0007010 cytoskeleton organization

GO:0030036 actin cytoskeleton organization

GOTERM_BP_FAT

GO:0032956 regulation of actin cytoskeleton organization

GOTERM_BP_FAT GOTERM_BP_FAT

GOTERM_BP_FAT

GOTERM_BP_FAT

GOTERM_BP_FAT

GOTERM_BP_FAT

GOTERM_BP_FAT

GO:0030029 actin filament-based process

GO:0032970 regulation of actin filament-based process

GO:0051493 regulation of cytoskeleton organization

GO:0043254 regulation of protein complex assembly

GO:0007015 actin filament organization

GO:0044087 regulation of cellular component biogenesis

GO:0043623 cellular protein complex assembly

11

Annotation Cluster 13

GOTERM_BP_FAT

GOTERM_BP_FAT

GOTERM_BP_FAT

GOTERM_BP_FAT

GOTERM_BP_FAT

GOTERM_BP_FAT

GOTERM_BP_FAT

GOTERM_BP_FAT

GOTERM_BP_FAT

\section{Enrichment Score: 2,165151910246645}

GO:0006915 apoptotic process

GO:0012501 programmed cell death

GO:0008219 cell death

GO:0043065 positive regulation of apoptotic process

GO:0043068 positive regulation of programmed cell death

GO:0042981 regulation of apoptotic process

GO:0043067 regulation of programmed cell death

GO:0010942 positive regulation of cell death

GO:0010941 regulation of cell death
39

28

16

11

17

12

11

10

16

12

$\begin{array}{ccc}20,31 & 0,0000006 & 6,22 \\ 14,58 & 0,0000333 & 4,48 \\ 8,33 & 0,0005232 & 3,28 \\ 5,73 & 0,0008570 & 3,07 \\ 8,85 & 0,0013675 & 2,86 \\ 5,73 & 0,0022626 & 2,65 \\ 6,25 & 0,0039288 & 2,41 \\ 5,73 & 0,0056071 & 2,25 \\ 5,21 & 0,0073000 & 2,14 \\ 8,33 & 0,0174964 & 1,76 \\ 6,25 & 0,0328116 & 1,48\end{array}$

33

$17,19 \quad 0,0006793$

$17,71 \quad 0,0008926$

$18,23 \quad 0,0011554$

$7,29 \quad 0,0056338$

$7,29 \quad 0,0060475$

$13,02 \quad 0,0077278$

$13,02 \quad 0,0086204$

$7,29 \quad 0,0090671$

$13,02 \quad 0,0181630$

3,17

3,05

2,94

2,25

2,22

2,11

2,06

2,04

1,74 


\begin{tabular}{lll}
\hline Category & Term & \\
\hline GOTERM_BP_FAT & GO:0044764 & multi-organism cellular process \\
GOTERM_BP_FAT & GO:0044403 & $\begin{array}{l}\text { symbiosis; encompassing mutualism } \\
\text { through parasitism }\end{array}$ \\
GOTERM_BP_FAT & GO:0044419 & $\begin{array}{l}\text { interspecies interaction between } \\
\text { organisms }\end{array}$ \\
GOTERM_BP_FAT & GO:0050792 & regulation of viral process \\
GOTERM_BP_FAT & GO:0043903 & regulation of symbiosis; encompassing \\
mutualism through parasitism
\end{tabular}

\begin{tabular}{|c|c|c|c|}
\hline Count & $\%$ & $P$ value & $\begin{array}{l}-\log _{10} \\
P \text { value }\end{array}$ \\
\hline 24 & 12,50 & 0,0001328 & 3,88 \\
\hline 24 & 12,50 & 0,0001925 & 3,72 \\
\hline 24 & 12,50 & 0,0001925 & 3,72 \\
\hline 10 & 5,21 & 0,0014392 & 2,84 \\
\hline 10 & 5,21 & 0,0025537 & 2,59 \\
\hline 10 & 5,21 & 0,0149688 & 1,82 \\
\hline 11 & 5,73 & 0,0179019 & 1,75 \\
\hline 15 & 7,81 & 0,0003327 & 3,48 \\
\hline
\end{tabular}

Annotation Cluster 15 Enrichment Score: 1,9941548315648696

GOTERM_BP_FAT GO:0010608 posttranscriptional regulation of gene expression

Annotation Cluster 16 Enrichment Score: 1,9755340900925409

$\begin{array}{lll}\text { GOTERM_BP_FAT } & \text { GO:0098609 } & \text { cell-cell adhesion } \\ \text { GOTERM_BP_FAT } & \text { GO:0007155 } & \text { cell adhesion } \\ \text { GOTERM_BP_FAT } & \text { GO:0022610 } & \text { biological adhesion }\end{array}$

22

28

28

11,46

$14,58 \quad 0,0124756$

$14,58 \quad 0,0130251$

2,14

1,90

1,89

Annotation Cluster 17 Enrichment Score: 1,959411589145368

GOTERM_BP_FAT

GOTERM_BP_FAT

negative regulation of protein metabolic process

GOTERM_BP_FAT

GO:0032269 negative regulation of cellular protein metabolic process

GO:0010605 negative regulation of macromolecule metabolic process

GOTERM_BP_FAT

GOTERM_BP_FAT

GOTERM_BP_FAT

GO:0031324 negative regulation of cellular metabolic process

GO:0009892 negative regulation of metabolic process

GO:0031400 negative regulation of protein

modification process

Annotation Cluster 18 Enrichment Score: 1,936584758141524

GOTERM_BP_FAT

GOTERM_BP_FAT

GOTERM_BP_FAT

GOTERM_BP_FAT

GO:0016050 vesicle organization

GO:0051656 establishment of organelle localization

GO:0051640 organelle localization

GO:0061024 membrane organization

Annotation Cluster 19 Enrichment Score: 1,8795156753917819

GOTERM_BP_FAT

GO:0044772 mitotic cell cycle phase transition

GOTERM_BP_FAT

GOTERM_BP_FAT

GOTERM_BP_FAT

GOTERM_BP_FAT

GO:0044770 cell cycle phase transition

GO:0007346 regulation of mitotic cell cycle

GO:0051726 regulation of cell cycle

GO:0010564 regulation of cell cycle process

Annotation Cluster 20 Enrichment Score: 1,8009512240557284

GOTERM_BP_FAT

GOTERM_BP_FAT

GOTERM_BP_FAT

GOTERM_BP_FAT

GOTERM_BP_FAT

GOTERM_BP_FAT

GO:0006928 movement of cell or subcellular component

GO:0001667 ameboidal-type cell migration

GO:0016477 cell migration

GO:0040011 locomotion

GO:0051674 localization of cell

GO:0048870 cell motility
24

23

40

39

41

14

14

$12,50 \quad 0,0003924$

$11,98 \quad 0,0004541$

$20,83 \quad 0,0009171$

$20,31 \quad 0,0020281$

$21,35 \quad 0,0022467$

$7,29 \quad 0,0061331$

2,65

2,21
11

12

13

19

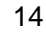

14

13

19

13

36

11

23

27

24

24

10

$\begin{array}{lll}5,73 & 0,0008793 & 3,06 \\ 6,25 & 0,0037914 & 2,42 \\ 6,77 & 0,0042647 & 2,37 \\ 9,90 & 0,0166639 & 1,78\end{array}$

$\begin{array}{lll}7,29 & 0,0022892 & 2,64 \\ 7,29 & 0,0038923 & 2,41 \\ 6,77 & 0,0044042 & 2,36 \\ 9,90 & 0,0103361 & 1,99 \\ 6,77 & 0,0183945 & 1,74\end{array}$

$18,75 \quad 0,0001080$

3,97

$5,73 \quad 0,0016181$

2,79

$11,98 \quad 0,0042877$

2,37

$14,06 \quad 0,0062275 \quad 2,21$

$12,50 \quad 0,0082302 \quad 2,08$

$12,50 \quad 0,0082302 \quad 2,08$
Annotation Cluster 22 Enrichment Score: 1,6759945901312603

GOTERM_BP_FAT GO:0051098 regulation of binding

$5,21 \quad 0,0035997$

2,44 


\begin{tabular}{|c|c|c|c|c|c|c|}
\hline Category & Term & & Count & $\%$ & $P$ value & $\begin{array}{l}-\log _{10} \\
P \text { value }\end{array}$ \\
\hline GOTERM_BP_FAT & GO:0016050 & vesicle organization & 11 & 5,73 & 0,0008793 & 3,06 \\
\hline Annotation Cluster 24 & \multicolumn{6}{|c|}{ Enrichment Score: 1,6676421778588604 } \\
\hline GOTERM_BP_FAT & GO:0010605 & $\begin{array}{l}\text { negative regulation of macromolecule } \\
\text { metabolic process }\end{array}$ & 40 & 20,83 & 0,0009171 & 3,04 \\
\hline GOTERM_BP_FAT & GO:0016070 & RNA metabolic process & 66 & 34,38 & 0,0018345 & 2,74 \\
\hline GOTERM_BP_FAT & GO:0031324 & $\begin{array}{l}\text { negative regulation of cellular metabolic } \\
\text { process }\end{array}$ & 39 & 20,31 & 0,0020281 & 2,69 \\
\hline GOTERM_BP_FAT & GO:0009892 & negative regulation of metabolic process & 41 & 21,35 & 0,0022467 & 2,65 \\
\hline GOTERM_BP_FAT & GO:0010467 & gene expression & 72 & 37,50 & 0,0033792 & 2,47 \\
\hline GOTERM_BP_FAT & GO:1903508 & $\begin{array}{l}\text { positive regulation of nucleic acid- } \\
\text { templated transcription }\end{array}$ & 25 & 13,02 & 0,0049290 & 2,31 \\
\hline GOTERM_BP_FAT & GO:0045893 & $\begin{array}{l}\text { positive regulation of transcription; DNA- } \\
\text { templated }\end{array}$ & 25 & 13,02 & 0,0049290 & 2,31 \\
\hline GOTERM_BP_FAT & GO: 1902680 & $\begin{array}{l}\text { positive regulation of RNA biosynthetic } \\
\text { process }\end{array}$ & 25 & 13,02 & 0,0059035 & 2,23 \\
\hline GOTERM_BP_FAT & GO:0071310 & cellular response to organic substance & 35 & 18,23 & 0,0068966 & 2,16 \\
\hline GOTERM_BP_FAT & GO:0051254 & $\begin{array}{l}\text { positive regulation of RNA metabolic } \\
\text { process }\end{array}$ & 25 & 13,02 & 0,0087650 & 2,06 \\
\hline GOTERM_BP_FAT & GO:0070887 & cellular response to chemical stimulus & 40 & 20,83 & 0,0091588 & 2,04 \\
\hline GOTERM_BP_FAT & GO:0045944 & $\begin{array}{l}\text { positive regulation of transcription from } \\
\text { RNA polymerase II promoter }\end{array}$ & 20 & 10,42 & 0,0094253 & 2,03 \\
\hline GOTERM_BP_FAT & GO:2000113 & $\begin{array}{l}\text { negative regulation of cellular } \\
\text { macromolecule biosynthetic process }\end{array}$ & 23 & 11,98 & 0,0149422 & 1,83 \\
\hline GOTERM_BP_FAT & GO:0010629 & negative regulation of gene expression & 25 & 13,02 & 0,0152244 & 1,82 \\
\hline GOTERM_BP_FAT & GO:0006366 & $\begin{array}{l}\text { transcription from RNA polymerase II } \\
\text { promoter }\end{array}$ & 29 & 15,10 & 0,0164142 & 1,78 \\
\hline GOTERM_BP_FAT & GO:0010558 & $\begin{array}{l}\text { negative regulation of macromolecule } \\
\text { biosynthetic process }\end{array}$ & 24 & 12,50 & 0,0167612 & 1,78 \\
\hline GOTERM_BP_FAT & GO:0051172 & $\begin{array}{l}\text { negative regulation of nitrogen } \\
\text { compound metabolic process }\end{array}$ & 25 & 13,02 & 0,0177728 & 1,75 \\
\hline GOTERM_BP_FAT & GO:0010557 & $\begin{array}{l}\text { positive regulation of macromolecule } \\
\text { biosynthetic process }\end{array}$ & 26 & 13,54 & 0,0191004 & 1,72 \\
\hline GOTERM_BP_FAT & GO:0051171 & $\begin{array}{l}\text { regulation of nitrogen compound } \\
\text { metabolic process }\end{array}$ & 58 & 30,21 & 0,0211689 & 1,67 \\
\hline GOTERM_BP_FAT & GO:0010033 & response to organic substance & 40 & 20,83 & 0,0242937 & 1,61 \\
\hline GOTERM_BP_FAT & GO:0031327 & $\begin{array}{l}\text { negative regulation of cellular } \\
\text { biosynthetic process }\end{array}$ & 24 & 12,50 & 0,0261876 & 1,58 \\
\hline GOTERM_BP_FAT & GO:0045935 & $\begin{array}{l}\text { positive regulation of nucleobase- } \\
\text { containing compound metabolic process }\end{array}$ & 26 & 13,54 & 0,0286900 & 1,54 \\
\hline GOTERM_BP_FAT & GO:0006357 & $\begin{array}{l}\text { regulation of transcription from RNA } \\
\text { polymerase II promoter }\end{array}$ & 28 & 14,58 & 0,0305811 & 1,51 \\
\hline GOTERM_BP_FAT & GO:0009890 & $\begin{array}{l}\text { negative regulation of biosynthetic } \\
\text { process }\end{array}$ & 24 & 12,50 & 0,0307789 & 1,51 \\
\hline GOTERM_BP_FAT & GO:0071495 & $\begin{array}{l}\text { cellular response to endogenous } \\
\text { stimulus }\end{array}$ & 20 & 10,42 & 0,0318812 & 1,50 \\
\hline GOTERM_BP_FAT & GO:0010628 & positive regulation of gene expression & 26 & 13,54 & 0,0354523 & 1,45 \\
\hline GOTERM_BP_FAT & GO:0010468 & regulation of gene expression & 56 & 29,17 & 0,0357412 & 1,45 \\
\hline GOTERM_BP_FAT & GO:0034645 & $\begin{array}{l}\text { cellular macromolecule biosynthetic } \\
\text { process }\end{array}$ & 63 & 32,81 & 0,0383441 & 1,42 \\
\hline GOTERM_BP_FAT & GO:0031328 & $\begin{array}{l}\text { positive regulation of cellular biosynthetic } \\
\text { process }\end{array}$ & 26 & 13,54 & 0,0441184 & 1,36 \\
\hline Annotation Cluster 26 & Enrichment S & core: 1,6267402762137324 & & & & \\
\hline GOTERM_BP_FAT & GO:0007015 & actin filament organization & 10 & 5,21 & 0,0073000 & 2,14 \\
\hline Annotation Cluster 27 & Enrichment S & core: 1,573279514658293 & & & & \\
\hline GOTERM_BP_FAT & GO:0044265 & cellular macromolecule catabolic process & 20 & 10,42 & 0,0048455 & 2,31 \\
\hline GOTERM_BP_FAT & GO:0009057 & macromolecule catabolic process & 22 & 11,46 & 0,0102123 & 1,99 \\
\hline GOTERM_BP_FAT & GO:0044257 & cellular protein catabolic process & 14 & 7,29 & 0,0234922 & 1,63 \\
\hline GOTERM_BP_FAT & GO:0006511 & $\begin{array}{l}\text { ubiquitin-dependent protein catabolic } \\
\text { process }\end{array}$ & 12 & 6,25 & 0,0335224 & 1,47 \\
\hline GOTERM_BP_FAT & GO:0051603 & $\begin{array}{l}\text { proteolysis involved in cellular protein } \\
\text { catabolic process }\end{array}$ & 13 & 6,77 & 0,0358169 & 1,45 \\
\hline GOTERM_BP_FAT & GO:0019941 & $\begin{array}{l}\text { modification-dependent protein catabolic } \\
\text { process }\end{array}$ & 12 & 6,25 & 0,0364752 & 1,44 \\
\hline
\end{tabular}




\begin{tabular}{|c|c|c|c|c|c|c|}
\hline Category & Term & & Count & $\%$ & $P$ value & $\begin{array}{l}-\log _{10} \\
P \text { value }\end{array}$ \\
\hline GOTERM_BP_FAT & GO:0030163 & protein catabolic process & 15 & 7,81 & 0,0367867 & 1,43 \\
\hline GOTERM_BP_FAT & GO:0043632 & $\begin{array}{l}\text { modification-dependent macromolecule } \\
\text { catabolic process }\end{array}$ & 12 & 6,25 & 0,0396070 & 1,40 \\
\hline
\end{tabular}

Annotation Cluster 28 Enrichment Score: 1,556576769515713

$\begin{array}{lll}\text { GOTERM_BP_FAT } & \text { GO:0048534 } & \begin{array}{l}\text { hematopoietic or lymphoid organ } \\ \text { development }\end{array} \\ \text { GOTERM_BP_FAT } & \text { GO:0002520 } & \text { immune system development }\end{array}$

GOTERM_BP_FAT GO:0030097 haemopoiesis

$\begin{array}{llll}16 & 8,33 & 0,0129480 & 1,89 \\ 16 & 8,33 & 0,0200911 & 1,70 \\ 14 & 7,29 & 0,0382189 & 1,42\end{array}$

Annotation Cluster 29 Enrichment Score: 1,5536345503696056

GOTERM_BP_FAT GO:0016071 mRNA metabolic process

GOTERM_BP_FAT GO:0008380 RNA splicing

GOTERM_BP_FAT GO:0006396 RNA processing

$\begin{array}{llll}14 & 7,29 & 0,0196111 & 1,71 \\ 10 & 5,21 & 0,0211074 & 1,68 \\ 17 & 8,85 & 0,0228503 & 1,64\end{array}$

Annotation Cluster 31 Enrichment Score: 1,433959843797708

GOTERM_BP_FAT GO:0071407 cellular response to organic cyclic

$13 \quad 6,77 \quad 0,0068716 \quad 2,16$

GOTERM_BP_FAT compound

GOTERM_BP_FAT

GO:1901699 cellular response to nitrogen compound

GOTERM_BP_FAT

GO:1901701 cellular response to oxygen-containing

$13 \quad 6,77 \quad 0,0116311 \quad 1,93$

$\begin{array}{llll}17 & 8,85 & 0,0232646 & 1,63\end{array}$

GOTERM_BP_FAT

GO:0032870 cellular response to hormone stimulus

13

$\begin{array}{lll}6,77 & 0,0233614 & 1,63\end{array}$

GO:0071495 cellular response to endogenous stimulus

GOTERM_BP_FAT

GO:1901698 response to nitrogen compound

$20 \quad 10,42 \quad 0,0318812 \quad 1,50$

$16 \quad 8,33 \quad 0,0389384 \quad 1,41$

Annotation Cluster 32 Enrichment Score: 1,4086294256407783

GOTERM BP FAT GO:0031175 neuron projection development

GOTERM_BP_FAT GO:0030030 cell projection organization

GOTERM_BP_FAT

GO:0048666 neuron development

GOTERM_BP_FAT

GO:2000026 regulation of multicellular organismal development

$\begin{array}{cccc}18 & 9,38 & 0,0039748 & 2,40 \\ 23 & 11,98 & 0,0124018 & 1,91 \\ 18 & 9,38 & 0,0189116 & 1,72 \\ 27 & 14,06 & 0,0252845 & 1,60\end{array}$

Annotation Cluster 36 Enrichment Score: 1,3372413426913092

\begin{tabular}{|c|c|c|c|c|c|c|}
\hline GOTERM_BP_FAT & GO:0002768 & $\begin{array}{l}\text { immune response-regulating cell surface } \\
\text { receptor signalling pathway }\end{array}$ & 11 & 5,73 & 0,0076645 & 2,12 \\
\hline GOTERM_BP_FAT & GO:0002764 & $\begin{array}{l}\text { immune response-regulating signalling } \\
\text { pathway }\end{array}$ & 13 & 6,77 & 0,0080526 & 2,09 \\
\hline GOTERM_BP_FAT & GO:0002757 & $\begin{array}{l}\text { immune response-activating signal } \\
\text { transduction }\end{array}$ & 11 & 5,73 & 0,0308856 & 1,51 \\
\hline GOTERM_BP_FAT & GO:0002682 & regulation of immune system process & 22 & 11,46 & 0,0395641 & 1,40 \\
\hline GOTERM_BP_FAT & GO:0006955 & immune response & 24 & 12,50 & 0,0415212 & 1,38 \\
\hline GOTERM_BP_FAT & GO:0031347 & regulation of defence response & 13 & 6,77 & 0,0429616 & 1,37 \\
\hline Annotation Cluster 37 & \multicolumn{6}{|c|}{ Enrichment Score: 1,3324823788934246 } \\
\hline GOTERM_BP_FAT & GO:0097190 & apoptotic signalling pathway & 14 & 7,29 & 0,0065757 & 2,18 \\
\hline
\end{tabular}

Figure A-6 shows the graphic representation of Table A-2 with terms including a p-value below 0.001 . 
posttranscriptional regulation of gene expression macromolecular complex subunit organization macromolecular complex assembly protein complex assembly protein complex biogenesis cellular component biogenesis protein complex subunit organization cellular component assembly cell cycle mitotic cell cycle cell cycle process mitotic cell cycle process mitotic nuclear division nuclear division

positive regulation of molecular function intracellular signal transduction regulation of hydrolase activity positive regulation of catalytic activity regulation of molecular functionpositive regulation of hydrolase activity regulation of GTPase activity regulation of signal transduction regulation of cell communicationregulation of signalling signal transduction by protein phosphorylation positive regulation of GTPase activity protein phosphorylation small GTPase mediated signal transduction cellular localization
localization in cell intracellular transport protein localization to nucleus protein import

protein import into nucleus protein targeting to nucleus single-organism nuclear import nuclear import single-organism intracellular transport cellular protein localization cellular macromolecule localization protein localization single-organism cellular localization protein localization to organellemacromolecule localization

Ras protein signal transduction regulation of GTPase activitypositive regulation of GTPase activity

chromosome organization negative regulation of cellular component organization negative regulation of organelle organization

DNA metabolic process

intracellular signal transduction cellular response to stress

signal transduction by protein phosphorylation protein phosphorylation

single-organism organelle organization cytoskeleton organization actin cytoskeleton organization regulation of actin cytoskeleton organization apoptotic process programmed cell death viral process multi-organism cellular process interspecies interaction between organisms symbiosis; encompassing mutualism through parasitism posttranscriptional regulation of gene expression negative regulation of protein metabolic process negative regulation of cellular protein metabolic process negative regulation of macromolecule metabolic process vesicle organization movement of cell or subcellular component vesicle organization negative regulation of macromolecule metabolic process
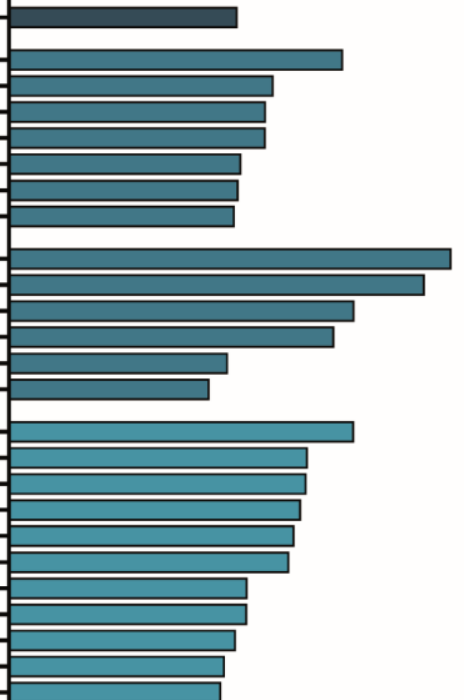

$+2$

$+2$
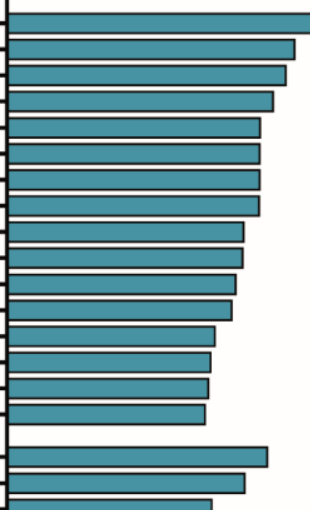

\begin{tabular}{|c|}
\hline \\
\hline 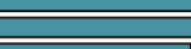 \\
\hline
\end{tabular}

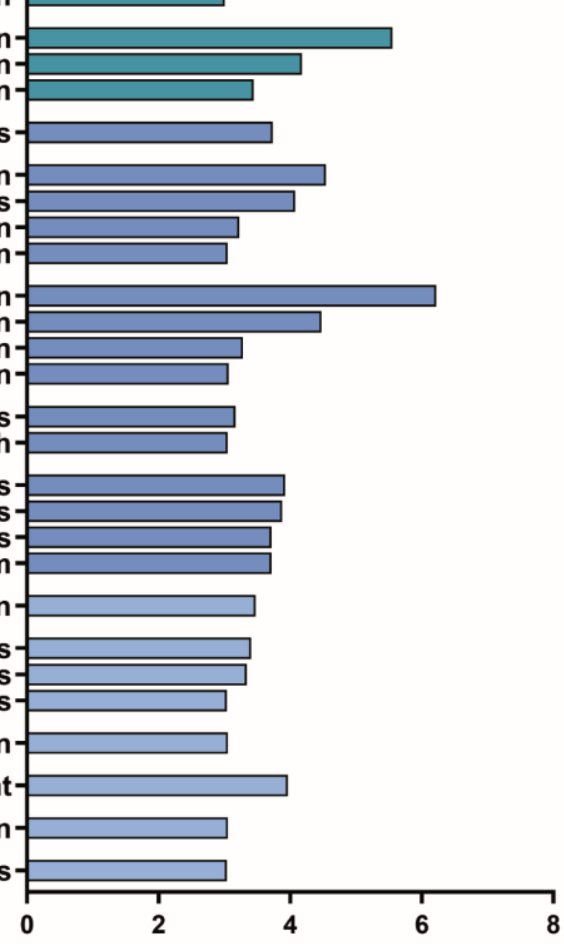

$-\log _{10} P$ value

Figure A-6: GO annotation of phosphoproteins changed by IL10+CpG stimulation

Terms of Table A-2 with a p-value below 0.001 are displayed. 